UNIVERSIDADE DE SÃO PAULO

ESCOLA DE ENGENHARIA DE SÃO CARLOS

IVÁN DARÍO GÓMEZ ARAÚJO

\title{
ANÁLISE MODAL OPERACIONAL: \\ MÉTODOS DE IDENTIFICAÇÃO BASEADOS EM TRANSMISSIBILIDADE.
}

São Carlos, São Paulo

2015 



\section{ANÁLISE MODAL OPERACIONAL: MÉTODOS DE IDENTIFICAÇÃO BASEADOS EM TRANSMISSIBILIDADE.}

Tese apresentada ao Departamento de Engenharia de Estruturas da EESC-USP como parte dos requisitos para a obtenção do título de Doutor em Engenharia Civil.

Área de concentração: Estruturas

Orientador: Prof. Titular Jose Elias Laier

Programa de Pós-graduação em estruturas VERSÃO CORREGIDA

São Carlos

A versão original encontra-se 2015. disponível no programa 


\section{AUTORIZO A REPRODUÇÃO TOTAL OU PARCIAL DESTE TRABALHO, POR QUALQUER MEIO COONVENCIONAL OU ELETRÔNICO, PARA FINS DE ESTUDO E PESQUISA, DESDE QUE CITADAA FONTE.}

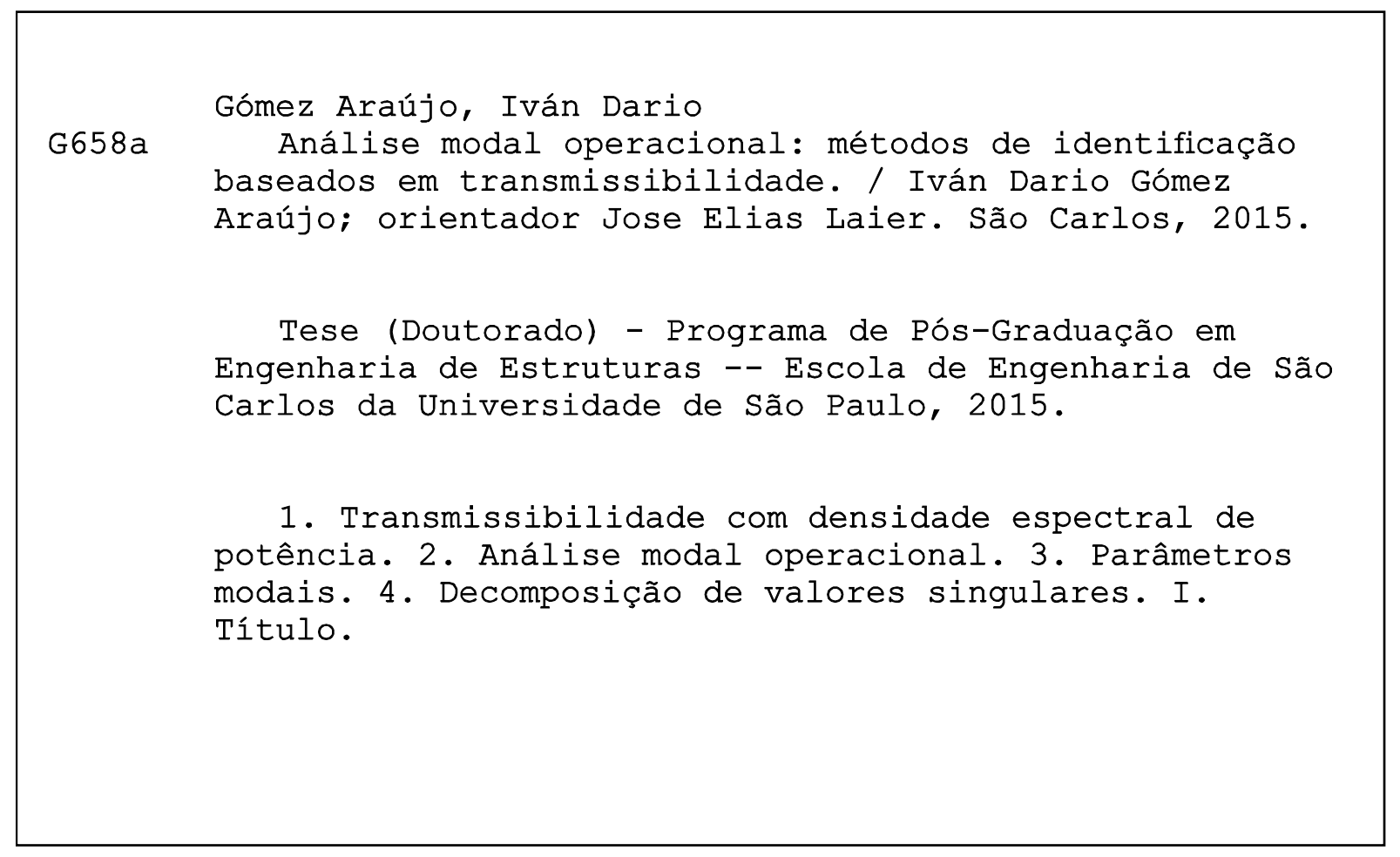




\section{FOLHA DE JULGAMENTO}

Candidato: Engenheiro IVÁN DARÍO GÓMEZ ARAÚJO.

Título da tese: "Análise modal operacional: métodos de idenlificação baseado em transmissibilidade".

Data da defesa: $25 / 02 / 2015$

\section{Comissão Julgadora:}

Resultado:

Prof. Titular José Ellas Laler (Orientador)

(Escola de Engenharia de Säo Carlos/EESC)

Prof. Dr. Ricardo Carrazedo

(Escola de Engenharia de São Carlos/EESC)

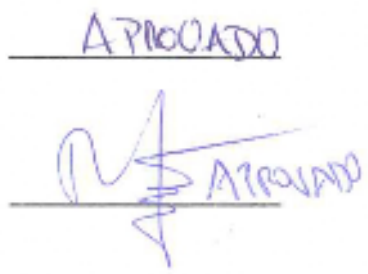

Prof. Dr. Leopoldo Pisanelli Rodrigues de Oliveira [Escola de Engenharia de São Carlos/EESC)

Prof. Dr. Walter Libardi

(Universidade Federal de São Carlos/UFSCar)

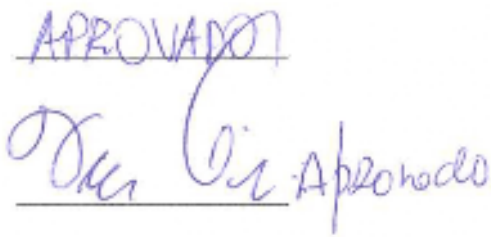

Prof. Dr. Joäo Antonio Pereira (Universidade Estadual Paulista "Júlio de Mesquita Filho"/UNESP - Itha Solteira)

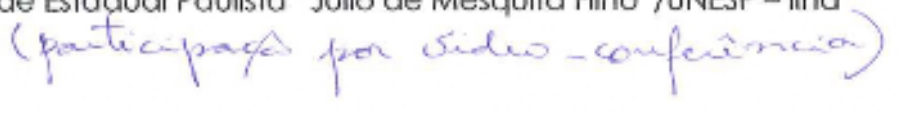

Coordenadora do Programa de Pós-Graduaçāo em Engenharia Civil (Engenharia de Estruturas):

Profa. Associada Ana Lucia Homce de Cresce El Debs

Presidente da Comissāo de Pós-Graduaçāo:

Prof. Associado Paulo César Lima Seganiline 

Dedico este trabajo a mis padres Olinda y Libardo.

A mis hermanas Anny y Kris.

Y a mi sobrino Matias 


\section{AGRADECIMENTOS}

Ao Prof. Dr. Jose Elias Laier por ter-me dado a confiança e a oportunidade de trabalhar sob sua orientação.

À profa. Dra Esperanza Maldonado (In memoriam) e ao prof. Dr Gustavo Chio Cho por ter-me orientado em meus primeiros passos como pesquisador.

À minha família na Colômbia por seu grande apoio desde a distancia.

À minha namorada Indara pelo seu amor e seu apoio durante o tempo que temos estado juntos. Igualmente, à família Soto Izquierdo pelo carinho dado.

A todos os amigos e colegas que me acompanharam durante estes anos no Brasil, especialmente ao Beto, Carolina, Dorival, Felipe, Marcela, Jesus Villalba, Jesus Garcia, Jose, Pilar e Rafael.

A todos os funcionários do Departamento de Estruturas da Escola de Engenharia de São Carlos da Universidade de São Paulo pela colaboração.

À CAPES e CNPq pela bolsa de estudo. 

Yo quiero seguir jugando a lo perdido yo quiero ser a la zurda más que diestro Dirán que pasó de moda la locura Dirán que la gente es mala y no merece más yo seguiré soñando travesuras.

Silvio Rodríguez (EL NECIO) 


\section{RESUMO}

GÓMEZ, I. D. (2015) Análise modal operacional: métodos de identificação baseados em transmissibilidade. Tese (Doutorado)- Escola de Engenharia de São Carlos, Universidade de São Paulo, São Carlos, 2015.

O presente trabalho tem como objetivo desenvolver novas alternativas de identificação modal para estruturas sob excitações em condição de operação baseadas em funções de transmissibilidade. Recentes metodologias formuladas sobre conceitos de transmissibilidade têm surgido como alternativa para a identificação de parâmetros modais de estruturas. A identificação nestas metodologias é independente do espectro da excitação, sendo uma vantagem importante com respeito a metodologias anteriores no domínio da frequência que supõem a excitação como ruído branco. Dessa forma, aproveitando os diferentes trabalhos dirigidos a avaliar parâmetros modais com uso da transmissibilidade, são propostas três novas alternativas. A primeira delas propõe a decomposição de valores singulares sobre matrizes de funções de transmissibilidade escalar com densidade espectral para estimar frequências naturais e modos de vibração. A segunda alternativa propõe o conceito de funções de transmissibilidade multivariável com diferente referência para a identificação modal. E a terceira introduz uma melhora na primeira alternativa incluindo a possibilidade da estimação de taxas de amortecimento. Uma ferramenta computacional para a análise modal é desenvolvida como apoio para as simulações numéricas de verificação das metodologias de identificação modal propostas. Diferentes exemplos numéricos com uma viga submetida a excitações de ruído colorido mostram que os métodos propostos são capazes de identificar parâmetros modais sem a introdução das frequências adicionais devido às excitações de ruído colorida utilizadas. Além disso, os dados de um teste de vibrações sobre uma ponte em operação foram utilizados para verificar os métodos.

Palavras chaves: Transmissibilidade com densidade espectral de potência, análise modal operacional, parâmetros modais, decomposição de valores singulares. 



\section{ABSTRACT}

GÓMEZ, I. D. (2015) Operational modal analysis: identification methods based on transmissibility. Doctoral Thesis - School of Engineering of São Carlos, University of São Paulo, São Carlos, 2015.

This research aims to develop new alternatives of modal identification for structures under excitation in operation condition based on transmissibility functions. Latest methodologies based on transmissibility concepts have been arising as alternatives for modal parameter identification of structures. Modal parameter identification in this type methodology is input spectrum independent being an important advantage with respect previous frequency domain methods that assumes white noise excitation. Different alternatives of modal identification based on transmissibility functions are proposed in this work. The first of them proposes singular value decomposition on scalar transmissibility functions matrices with spectral density to estimate natural frequencies and vibration modes (PSDTM-SVD method). A second alternative proposes the concept of multivariable transmissibility functions with different transferring outputs for modal parameter identification. And the third alternative proposes an enhanced PSDTM-SVD method, which permits to identify modal damping. Computational tool for modal analysis is developed as a support for the numerical simulations of verification of modal identification methodologies proposed. Different numerical examples of a beam model subjected to colored noise excitations show that the proposed methods are capable of identifying modal parameters without the introduction of the additional frequencies due to the excitations used. Furthermore, data from an operational vibration bridge test were used to verify the methods.

Keywords: Power spectrum density transmissibility, operational modal analysis, modal parameters, singular value decomposition. 



\section{SUMÁRIO}

CAPÍTULO 1.INTRODUÇÃO

$\begin{array}{lll}1.1 & \text { MOTIVAÇÃO DA PESQUISA } & 25\end{array}$

1.2 OBJETIVOS. 29

$\begin{array}{ll}\text { 1.2.1 Objetivo geral. } & 29\end{array}$

$\begin{array}{lll}1.2 .2 & \text { Objetivos específicos. } & 29\end{array}$

$\begin{array}{lll}1.3 & \text { CONTRIBUÇÕES DO TRABALHO } & 29\end{array}$

CAPÍTULO 2.FUNDAMENTOS ANALITÍCOS DA ANÁLISE MODAL OPERACIONAL. 33

2.1 VIBRAÇÕES ALEATÓRIAS 33

$\begin{array}{lll}2.2 & \text { RESPOSTA DE UM SISTEMA LTI-SDF } & 38\end{array}$

2.3 RESPOSTA DE UM SISTEMA LTI-MDF PARA O AMORTECIMENTO PROPORCIONAL. $\quad 42$

2.4 RESPOSTA DE UM SISTEMA LTI-MDF PARA O AMORTECIMENTO GERAL. 47

2.5 FUNÇÕES DE TRANSMISSIBILIDADE ESCALAR E MULTIVARIÁVEL 50

2.6 CONSIDERAÇÕES FINAIS. 53

CAPÍTULO 3.REVISÃO DE MÉTODOS NO DOMINIO DA FREQUÊNCIA PARA A ANÁLISE MODAL OPERACIONAL.

3.1 FUNÇÕES DE CORRELAÇÂO DE DADOS DE RESPOSTA 56

3.1.1 Método direto. 56

3.1.2 Método baseado no periodograma médio. 57

3.1.3 Método do decremento aleatório. 58

3.2 FUNÇÕES DE DENSIDADE ESPECTRAL. 61

3.3 MÉTODOS NÃO PARAMÉTRICOS NO DOMÍNIO DA FREQUÊNCIA. 64

$\begin{array}{lll}\text { 3.3.1 Método de seleção de picos (PP). } & 65\end{array}$

3.3.2 Método de decomposição do domínio da frequência (FDD) 68

$\begin{array}{ll}\text { 3.3.3 Método melhorado do FDD } & 70\end{array}$

$\begin{array}{ll}\text { 3.3.4 Métodos RD-FDD e RD-EFDD. } & 71\end{array}$

3.3.5 Método decomposição do domínio da frequência-espaço (FSDD) 72 
3.3.6 Método baseado em transmissibilidade.

3.3.7 Método baseado em transmissibilidade escalar com densidade espectral (PSDT).

3.4 MÉTODO PARAMÉTRICO NO DOMÍNIO DA FREQUÊNCIA. 78

3.5 CONSIDERAÇÕES FINAIS 83

CAPÍTULO 4.MÉTODO BASEADO EM MATRIZES DE TRANSMISSIBILIDADE COM $\begin{array}{ll}\text { DENSIDADE ESPECTRAL VIA SVD (PSDTM-SVD). } & 87\end{array}$

4.1 IDENTIFICAÇÃO DE PARÂMETROS MODAIS USANDO PSDTM-SVD 88

$\begin{array}{lll}4.2 & \text { EXEMPLO NUMÉRICO } & 92\end{array}$

$\begin{array}{lll}4.3 & \text { DADOS REAIS DE VIBRAÇÃO } & 97\end{array}$

4.3.1 Descrição da ponte. 99

4.3.2 Ensaio de vibrações 99

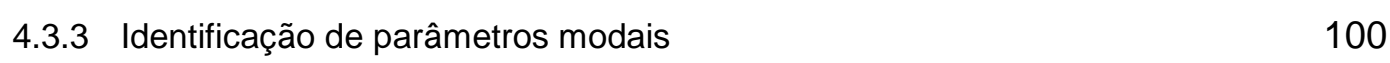

$\begin{array}{lll}4.4 & \text { CONSIDERAÇÕES FINAIS. } & 104\end{array}$

CAPÍTULO 5.TRANSMISSIBILIDADE MULTIVARIÁVEL COM DIFERENTES $\begin{array}{ll}\text { REFERÊNCIAS DAS SAÍDAS. } & 107\end{array}$

5.1 FUNÇÕES DE TRANSMISSIBILIDADE MULTIVARIÁVEL COM DIFERENTE $\begin{array}{ll}\text { REFERÊNCIA DA SAÍDA. } & 108\end{array}$

$\begin{array}{lll}5.2 & \text { PROPRIEDADE DA PSDT MULTIVARIÁVEL. } & 110\end{array}$

5.3 IDENTIFICAÇÃO DE PARÂMETROS MODAIS USANDO MULTIVARIÁVEL PSDT.

$\begin{array}{lll}5.4 & \text { EXEMPLO NUMÉRICO } & 114\end{array}$

$\begin{array}{lll}5.5 & \text { DADOS REAIS DE VIBRAÇÃO } & 122\end{array}$

$\begin{array}{lll}5.6 & \text { CONSIDERAÇÕES FINAIS } & 126\end{array}$

CAPÍTULO 6.MÉTODO PSDTM-SVD MELHORADO. 129

6.1 IDENTIFICAÇÃO DE PARÂMETROS MODAIS USANDO O MÉTODO PSDTM-SVD $\begin{array}{ll}\text { MELHORADO } & 130\end{array}$

6.2 IDENTIFICAÇÃO DO NÚMERO DE CARGAS NÃO CORRELACIONADAS. 133

$\begin{array}{lll}6.3 & \text { EXEMPLO NUMÉRICO. } & 134\end{array}$

$\begin{array}{lll}6.4 & \text { CONSIDERAÇÕES FINAIS } & 138\end{array}$ 
CAPÍTULO 7.USO DOS MÉTODOS DE IDENTIFICAÇÃO EM UMA CONDIÇÃO DE UMA ÚNICA CARGA.

7.1 TRANSMISSIBILIDADE ESCALAR E MULTIVARIÁVEL COM DENSIDADE ESPECTRAL PARA UMA ÚNICA CARGA.

7.2 IDENTIFICAÇÃO EM UMA CONDIÇÂO DE UMA ÚNICA CARGA.

7.3 EXEMPLOS NUMÉRICOS COM UMA ÚNICA CARGA.

7.4 CONSIDERAÇÕES FINAIS

CAPÍTULO 8.CONCLUSÕES E TRABALHOS FUTUROS

8.1 CONCLUSÕES

8.2 TRABALHOS FUTUROS

APÊNDICE A: FERRAMENTA COMPUTACIONAL PARA A ANÁLISE MODAL, MYFEM MODAL 2D.

A.1 DESCRIÇÃO GERAL DE MYFEM MODAL 2D.

A.2 EXEMPLOS DE USO DO PROGRAMA PARA A ANÁLISE MODAL TEÓRICO. 172

A.2.1 Funções de resposta em frequência (FRFs)

A.2.2 Funções de densidade espectral (PSDs) 178

A.2.3 Funções de transmissibilidade multivariável (FTs) 178

A.2.4 Funções de resposta impulsiva (FRIs) 179

A.2.5 Funções de correlação (FCs). 180

A.3 EXEMPLOS DE USO DO PROGRAMA PARA A ANÁLISE MODAL A PARTIR DE DADOS DE RESPOTA DO MODELO ESTRUTURAL.

182

A.3.1 Funções de correlação de dados de resposta. 184

A.3.2 Funções de densidade espectral de dados de resposta 186

A.3.3 Funções de transmissibilidade de dados de resposta. 188

A.3.4 Uso do método de seleção de picos para avaliação de parâmetros modais. 190 A.4 CONSIDERAÇÕES FINAIS 195 



\section{LISTA DE FIGURAS}

Figura 2-1 Densidade espectral da resposta, $\omega_{0}=2 \mathrm{rad} / \mathrm{s}, \xi=0.05$, adequada aproximação como um ruído branco.

Figura 2-2 Densidade espectral da resposta, $\omega_{0}=2 \mathrm{rad} / \mathrm{s}, \xi=0.15$, inadequada aproximação como um ruído branco.

Figura 2-3 Densidade espectral da resposta, $\omega_{0}=2 \mathrm{rad} / \mathrm{s}, \xi=0.15$, inclusão da frequência de excitação no espectro da saída.

Figura 2-4 Diagrama do sistema a identificar quando a excitação é ruído colorido. .42

Figura 2-5 Matriz de densidade espectral de resposta $[S(i \omega)]_{x_{k} x_{j}}$ de um sistema de três graus de liberdade com uma distribuição de excitação de ruído branco aplicado a) no segundo grau de liberdade e b) no primeiro grau de liberdade. .46

Figura 3-1 Seleção de segmentos, método do decremento aleatório. .59

Figura 3-2 Sistematização dos principais métodos na análise modal operacional no domínio da frequência .85

Figura 4-1 Diagrama de fluxo do método proposto PSDTM-SVD. .92

Figura 4-2 Características da excitação do exemplo numérico. .93

Figura 4-3 Resultados dos métodos a) FDD, b) PSDT, c) PSDTMSVD para a Carga 1. .93

Figura 4-4 Resultados dos métodos a) FDD, b) PSDT, c)PSDTMSVD para a Carga 2. .94

Figura 4-5 Decomposição em valores singulares da matriz de transmissibilidade $\left[T^{Z_{i}}{ }_{o_{0}} x_{1}(i \omega)\right] \ldots . .96$

Figura 4-6 a) O corte longitudinal, b) o corte transversal da superestrutura e c) o detalhe da planta da ponte Garcia Cadena. .98

Figura 4-7 Fotografia da ponte Garcia Cadena. .99

Figura 4-8 Configurações dos acelerômetros. 100

Figura 4-9 Decomposição em valores singulares das matrizes de transmissibilidade para a configuração 1 (setup 1) no eixo central da ponte, no vão B-7. 101

Figura 4-10 Resultados dos métodos a) FDD, b) PSDT, c) PSDTM-SVD para a configuração 1 (setup 1 ) sobre o eixo Central da ponte, no vão B-7.

Figura 4-11 Resultados dos métodos a) FDD, b) PSDT, c) PSDTM-SVD para a configuração 2 (setup 2) sobre o eixo Central da ponte, no vão B-7. 
Figura 4-12 Resultados dos métodos a) FDD, b) PSDT, c) PSDTM-SVD para a configuração 3 (setup

3 ) sobre o eixo Central da ponte, no vão B-7

Figura 4-13 Comparação das formas modais identificadas com os métodos FDD, PSDT e PSDTM-

SVD sobre o eixo Central da ponte, no vão B-7. 104

Figura 5-1 a) Exemplo da excitação, b) densidade espectral de potência da excitação usada no exemplo numérico. 115

Figura 5-2 Média normalizada das auto-densidades espectrais.

Figura 5-3 a),b) Decomposição de valores singulares e c), d) média de todos os valores na faixa de frequência de 0 até $175 \mathrm{~Hz}$ das matrizes definidas nas Equações (5-23) (Set 1) e (5-24) (Set 2), respectivamente. 118

Figura 5-4 Funções das matrizes a) $[\hat{T}(i \omega)]_{\text {set1 }}^{++}$e b) $[\hat{T}(i \omega)]_{\text {set } 2}^{++}$ 119

Figura 5-5 a) Diagrama de estabilidade e b) curva de ajuste usando o método PolyMAX aplicado na primeira linha da matriz $[\hat{T}(i \omega)]_{\text {set } 1}^{++}$ 120

Figura 5-6 a) Diagrama de estabilidade e b) curva de ajuste usando o método PolyMAX aplicado na primeira linha da matriz $[\hat{T}(i \omega)]_{\text {set } 2}^{++}$ 120

Figura 5-7 Estabilidade das taxas de amortecimento dos pólos estáveis extraídos da a) Figura 5-5 e b) Figura 5-6. 121

Figura 5-8 a) diagrama de estabilidade e b) estabilidade das taxas de amortecimento para os pólos estáveis determinados a partir do método PolyMAX aplicado na matriz de densidade espectral.

Figura 5-9 a), b) Decomposição de valores singulares e c), d) média dos valores singulares na faixa de frequência de 0 até $100 \mathrm{~Hz}$ para as matrizes de Set $\mathrm{A}$ e Set B, respectivamente. 124

Figura 5-10 a) diagrama de estabilidade e b) estabilidade das taxas de amortecimento para os pólos estáveis determinados usando o método PolyMAX na matriz inversa $[\hat{T}(i \omega)]_{s e t ~}^{++}$ 125

Figura 5-11 a) diagrama de estabilidade e b) estabilidade das taxas de amortecimento para os pólos estáveis determinados usando o método PolyMAX na matriz inversa $[\hat{T}(i \omega)]_{s e t B}^{++}$ 125

Figura 6-1 Identificação automática do número de cargas naõ correlacionadas presentes na estrutura. 134

Figura 6-2 Identificação do número máximo de valores singulares na matriz $\left[T^{z_{i}} x_{0} x_{5}(i \omega)\right]$. 135

Figura 6-3 a) Primeiros valores singulares das matrizes inversas b) Média ponderada dos primeiros valores singulares. 136 
Figura 6-4. a) Diagrama de estabilidade do método PolyMAX aplicado na função $\pi(i \omega)$ b) estabilidade das taxas de amortecimento dos pólos estáveis extraídos da Figura 6-4 a) 137

Figura 6-5 a) Faixa de frequências selecionada na função $\pi(i \omega)$ b) Transformada inversa de Fourier da faixa selecionada c) ajuste da envolvente da função em b) para avaliar o decremento logarítmico.

Figura 7-1 Cargas atuando simultaneamente no sistema. 146

Figura 7-2 Carga única mudando de posição no sistema. 146

Figura 7-3 Cargas deslocandose entre os graus de liberdade 5,8 e 11, Carga 1. 147

Figura 7-4 Carga impulsiva deslocando-se entre os graus de liberdade verticais, Carga 2. 147

Figura 7-5 a) diagrama de estabilidade e b) estabilidade das taxas de amortecimento para os pólos estáveis determinados usando o método PolyMAX na matriz inversa da Equação 5-24 (método com transmissibilidade multivariável). Caso de Carga 1.

Figura 7-6 a) diagrama de estabilidade e b) estabilidade das taxas de amortecimento para os pólos estáveis determinados usando o método PolyMAX na matriz inversa na matriz inversa da Equação 5-24 (método com transmissibilidade multivariável). Caso de Carga 2. 149

Figura 7-7 a) Diagrama de estabilidade do método PolyMAX aplicado na função resultante do métodos PSDTM-SVD melhorado e b) estabilidade das taxas de amortecimento dos pólos estáveis. Caso de Carga 1. 150

Figura 7-8 a) Diagrama de estabilidade do método PolyMAX aplicado na função resultante do métodos PSDTM-SVD melhorado e b) estabilidade das taxas de amortecimento dos pólos estáveis. Caso de Carga 2. 150

Figura A-1 Sistematização dos componentes principais do programa Myfem Modal $2 d$. 170

Figura A-2 Sistematização dos componentes para a análise modal a partir dados de resposta de Myfem Modal 2d..

Figura A-3 Viga de exemplo para a análise modal teórico e operacional. 172

Figura A-4 Criação do objeto Fem em Myfem Modal . 173

Figura A-5 Definição de características estruturais do modelo em Myfem Modal ..... .173

Figura A-6 Definição da análise dinâmica para a análise modal teórico em Myfem Modal 174

Figura A-7 Recuperação de dados de elementos da análise modal teórico em Myfem Modal . 175

Figura A-8 Método Mybode para o cálculo das FRFs. 176

Figura A-9 FRFs teóricas para os graus de liberdade 5 e 8, calculadas com Myfem Modal . 177 
Figura A-10 Método MyPSD2 para o cálculo das PSDs.

Figura A-11 PSDs teóricas para os graus de liberdade 5 e 8 para uma excitação de ruído branco localizada no grau de liberdade 11, calculadas com Myfem Modal . 178

Figura A-12 Método Mytransm para o cálculo das funções de transmissibilidade em múltiplos graus de liberdade. 179

Figura A-13 FTs teóricas entre os graus de liberdade 5, 8 e 17 com 11 e 14 com carregamento em 11 e 14, calculadas com Myfem Modal ..... 179

Figura A-14 Método Mylmpulse para o cálculo das funções de resposta impulsiva 180

Figura A-15 FRIs teóricas nos graus de liberdade 5 e 8, calculadas com Myfem Modal 180

Figura A-16 Método MyCorrelation para o cálculo das funções de correlação da resposta. 181

Figura A-17 FCs nos graus de liberdade 5 e 8 para uma entrada de ruído branco nos graus de liberdade 5 e 17, calculadas com Myfem Modal . 181

Figura A-18 Definição da análise dinâmica para a análise modal a partir de dados de resposta. ..... 183 Figura A-19 Informação do método noiseWG sobre o ruído branco gerado. 184

Figura A-20 Cálculo de funções de correlação de dados de resposta do modelo estrutural com Myfem Modal 2d.. 185

Figura A-21 Comparação das funções de correlação pelos métodos: direto, periodograma médio e decremento aleatório, com a função de correlação teórica. 186

Figura A-22 Cálculo de funções de densidade espectral de dados de resposta do modelo estrutural com Myfem Modal 2d. 187

Figura A-23 Comparação da função de densidade espectral teórica com as funções estimativas dos métodos de: Welch e Blackman e Tukey com as funções de decremento aleatório. 188

Figura A-24 Cálculo de funções de transmissibilidade de dados de resposta com Myfem Modal $2 d$.. 189

Figura A-25 Comparação da função de transmissibilidade estimada a partir de dados de resposta do modelo estrutural com a função de transmissibilidade teórica com Myfem Modal $2 d$. . . . 189

Figura A-26 Exemplo de uso do método de seleção de picos em Myfem Modal 2d. ......................... 190

Figura A-27 Média normalizado dos auto-espectros da matriz de densidade espectral. 191

Figura A-28 Uso do método Mytfmodos em Myfem Modal 2d. 192

Figura A-29 Informação do método Mytfmodos de Myfem Modal 2d. sobre as funções de transmissibilidade. 192

Figura A-30 Uso do método Myamortajuste em Myfem Modal 2d. 193

Figura A-31 Informação do método Myamortajuste de Myfem Modal 2d. 194 


\section{LISTA DE TABELAS}

Tabela 4-1 Frequências naturais identificadas para o exemplo .95

Tabela 4-2 Modos identificados com o método PSDTM-SVD 97

Tabela 4-3 Comparação dos modos teóricos e estimados com o método PSDTM-SVD 97

Tabela 4-4 Frequências naturais identificadas $(\mathrm{Hz})$ a partir das diferentes configurações de acelerômetros (Setups) sobre o eixo Central da ponte, no vão B-7.

Tabela 5-1 Frequências naturais amortecidas e taxas de amortecimento identificadas usando o método proposto para ambas as configurações Set 1 e Set 2.

Tabela 5-2 Modos de vibração identificados usando o método proposto para Set 2.

Tabela 5-3 Frequências naturais e taxas de amortecimento identificadas da ponte a partir do método proposto e o PolyMAX-PSD

Tabela 5-4 Modos de vibração identificados da ponte a partir do método proposto e o PolyMAX-PSD.

Tabela 6-1 Frequências naturais amortecidas e taxas de amortecimento identificadas usando o método PSDTM-SVD melhorado.

Tabela 7-1 Frequências naturais amortecidas e taxas de amortecimento identificadas usando o método com transmissibilidade multivariável.

Tabela 7-2 Frequências naturais amortecidas e taxas de amortecimento identificadas usando o método PSDTM-SVD melhorado.

Tabela A-1 Frequências naturais não amortecidas e amortecidas e taxas de amortecimentos do modelo de exemplo com o uso de Myfem Modal 175

Tabela A-2 Modos de vibração do exemplo com o uso de Myfem Modal . 176

Tabela A-3 Frequências de ressonância do modelo (teórico-identificado) 191

Tabela A-4 Formas modais identificadas. 192

Tabela A-5 Comparação de formas modais teóricas e identificadas. 193

Tabela A-6 Taxas de amortecimentos (teórico-identificado) 194 



\section{CAPÍTULO 1. INTRODUÇÃO}

\subsection{MOTIVAÇÃO DA PESQUISA}

A análise modal de estruturas consiste na determinação dos seus parâmetros modais (frequências naturais, formas modais, amortecimentos modais e fatores de participação modal) tendo-se por base a formulação de um modelo matemático do comportamento dinâmico. Esse tipo de análise tem sido amplamente utilizado na abordagem de problemas de vibração, como: modificação da dinâmica das estruturas, atualização de modelos analíticos, dimensionamento dinâmico ótimo e controle de vibrações. Outra área de pesquisa de grande interesse trata da determinação das características modais de uma estrutura como parte de um sistema de monitoramento para a análise da saúde estrutural e a detecção de danos que pode ser aplicável em diferentes setores industriais, tais como aeroespacial, mecânica, petróleos e civil. Dessa forma, duas abordagens têm sido desenvolvidas nas últimas décadas com o fim de determinar as características dinâmicas das estruturas: Análise Modal Tradicional (Tradicional Modal Analysis-TMA) e Análise Modal Operacional (Operational Modal Analysis-OMA).

As primeiras pesquisas em análise modal experimental foram feitas a partir da aproximação de TMA, na qual a estrutura é excitada (entrada) com forças dinâmicas e uma ou várias medições da resposta da estrutura (saídas) são registradas em termos das acelerações, velocidades ou deslocamentos decorrentes destas forças. Assim, em base a uma relação de entradas e saídas podem ser extraídos os parâmetros modais da estrutura em uma faixa de frequência de interesse. Baseando-se nesse conceito de entrada e saída têm-se desenvolvido numerosas técnicas ou métodos de identificação modal. Esses métodos são classificados de forma geral dependendo da sua formulação em métodos do domínio do tempo (TD) e do domínio da frequência (FD). Ainda, os métodos podem ser divididos, segundo o número de entradas e saídas disponíveis, como: uma entrada/uma saída (Single Input,Single Output-SISO), uma-entrada/múltiplas-saídas (Single Input, Multiple Output-SIMO) ou múltiplas-entradas/múltiplas-saídas (Multiple Input, Multiple Output-MIMO). Porém, a TMA tem algumas limitações que são apresentadas a seguir:

- Normalmente, a estrutura é excitada artificialmente com o intuito de se obterem as funções da resposta em frequência (FRFs), ou as funções de resposta impulsiva (FRIs). Para as medições realizadas em campo e em grandes estruturas, a aplicação desta metodologia se torna difícil devido à dificuldade de se medirem as FRFs e as FRIs. Tal dificuldade se origina pela existência de outros tipos de excitações além da excitação artificial e da necessidade do uso de grandes quantidades de energia, que muitas vezes não é disponível no caso de grandes estruturas. 
- A TMA é realizada, na maioria dos casos, no laboratório. Nesse sentido, as condições reais de operação de muitas aplicações industriais podem diferir significativamente das aplicadas no ensaio do laboratório. Além disso, apenas componentes são ensaiados geralmente e não sistemas completos.

A partir do começo da década de 90 a Análise Modal Operacional (OMA) tem sido usada para caracterização dinâmica de estruturas. OMA, também conhecido como análise de vibrações ambientais, excitação natural ou só saída (Output-Only), utiliza somente as medições de resposta da estrutura em condições de operação sujeita a excitações ambientais ou naturais (vento, tráfego, tremores, etc) para identificar as características modais. Existem diferentes vantagens a partir do seu uso mostradas em Zhang, Brincker. et al. (2005), como:

- É econômica e rápida de se realizar devido a que não precisa de equipamento para excitar a estrutura nem da simulação das condições de contorno.

- As características dinâmicas do sistema completo, e não somente de alguns componentes, podem ser obtidas, portanto, representam mais fielmente o sistema analisado.

- A realização dos ensaios experimentais não interfere com o uso normal ou operação da estrutura.

- É uma forma atrativa para fazer uma monitorização on-line da saúde estrutural para a detecção de danos baseada nas características dinâmicas como mostrado no trabalho de Magalhaes, Cunha et al. (2012).

Com as anteriores vantagens, este tipo de análise tem recebido uma grande atenção por parte da comunidade da engenharia civil com vistas às aplicações em plataformas de petróleo, prédios, torres e pontes (Brownjohn et al., 1989; Araujo, Maldonado e Cho, 2011; Gentile e Saisi, 2011; Liu et al., 2012; Liu, Loh e Ni, 2013). No entanto, uma das limitações do uso da Análise Modal Operacional com medições unicamente da resposta são as incertezas contidas nos dados, as quais podem ter origem de diferentes fontes, tais como as variações operacionais e ambientais da estrutura. Esses tipos de variações têm uma influência direta na resposta dinâmica da estrutura e são refletidas na avaliação dos parâmetros dinâmicos. Dessa forma, devido a que os dados podem ser medidos sob condições variáveis, existe a necessidade de normalizar os dados com o fim de estabelecer medições em ciclos similares de condições operacionais e ambientais, para assim facilitar uma comparação (Doebling et al., 1996).

Devido ao impacto econômico que pode representar esse tipo de técnica na análise dinâmica das estruturas e suas notáveis potencialidades para diferentes tipos de aplicações, 
tem-se observado nos últimos anos um grande incremento na atividade de pesquisa em torno às bases teóricas de OMA e suas possíveis aplicações em diferentes campos da engenharia. Assim, no ano 2005 celebrou-se a primeira versão do International Operational Modal Analysis Conference (IOMAC) e no ano 2010 o Journal Mechanical System and Signal Processing publicou uma edição especial sobre o tema (Brincker e Kirkegaard, 2010).

Além disso, um dos problemas importantes em OMA é a necessidade de se obterem parâmetros modais confiáveis, o que é possível através do desenvolvimento e da aplicação de técnicas robustas de identificação modal que utilizem somente dados de resposta. Entre as técnicas existentes para a identificação modal para OMA podem ser encontradas aquelas desenvolvidas no domínio do tempo, como por exemplo: Least Square Exponential (LSCE) (Vold et al., 1982), Eigensystem Realization Algorithm (ERA) (Juang e Pappa, 1985), Extend Ibrahim methods (EITD) (Fukuzono, 1986) e Stochastic Subspace Identification (SSI) (Overschee et al., 1996) entre outros. Já no domínio da frequência existem técnicas, como: Peak Picking (PP) (Felber, 1993), Frequency Domain Decomposition (FDD) (Brincker, Zhang e Andersen, 2000) e Frequency-Spatial Domain Decomposition (FSDD) (Zhang, Wang e Tamura, 2010) entre outros. Também, alguns trabalhos abordam uma comparação entre as potencialidades e limitações das anteriores metodologias como em Peeters e De Roeck (2001) e Andersen, Brincker et al. (1999).

A maioria dos métodos de identificação modal expressados anteriormente, principalmente, os métodos no domínio da frequência, com exceção de alguns métodos no domínio do tempo, como o método SSI, supõem que as excitações naturais ou ambientais de entrada à estrutura contêm uma ampla faixa de frequências de igual contribuição, tal que as principais frequências naturais da estrutura podem ser excitadas. Isso leva à suposição que as excitações são um processo aleatório do tipo ruído branco (White Noise) com um espectro em frequência constante. Porém, algumas forças atuam periodicamente, por exemplo, ondas e máquinas rotativas, contendo uma maior energia para frequências específicas. Isso também representa um problema para OMA, quando não existe nenhum conhecimento enquanto ao espectro do carregamento, o que não pode garantir que todas as frequências identificadas sejam relacionadas ao sistema de interesse.

Como consequências do problema anterior recentemente têm surgido metodologias de identificação modal no domínio da frequência para OMA baseadas em conceitos de transmissibilidade. Nesse tipo de metodologias para a análise modal não é necessário que a força de excitação seja considerada como ruído branco. A força pode ser arbitrária, como 
ruído colorido, varredura de senos ou impacto, etc., sempre que seja garantido que essas forças consigam excitar as frequências do sistema.

Em geral, as funções de transmissibilidade contêm pólos que diferem dos pólos do sistema, logo não podem ser usadas diretamente na identificação de parâmetros modais. No entanto, uma primeira proposta baseada no uso de funções de transmissibilidade para a análise modal foi dada por Devriendt e Guillaume (2007; 2008). A ideia essencial é que diferentes funções de transmissibilidade determinadas a partir de diferentes condições de carga convergem nos pólos do sistema para a mesma relação de modos de vibração. Portanto, a partir de realizar uma subtração entre duas funções de transmissibilidade que relacionam as mesmas saídas, mas que vêm de diferentes condições de carga pode-se encontrar que os pólos do sistema são zeros e pólos na inversa da função subtração. Como uma continuação do trabalho de Devriendt e Guillaume, mais recentemente têm sido publicados diferentes trabalhos que tentam aperfeiçoar a metodologia, mas todos baseados no uso de diferentes condições de carga para avaliação das funções de transmissibilidade, entre issos trabalhos podem-se citar Devriendt, De Sitter e Guillaume (2010), Devriendt et al. (2013), Weijtjens, Lataire, et al. (2014) e Weijtjens, De Sitter, et al. (2014).

Os trabalhos anteriormente mencionados podem identificar parâmetros modais pela combinação de funções de transmissibilidade calculadas a partir de diferentes condições de carga. No entanto, essas metodologias devem assegurar e satisfazer durante a experimentação a existência das diferentes condições de carga. Isso se poderia tornar em uma limitação no caso da análise modal de estruturas em operação, já que um préprocessamento dos dados é necessário para estabelecer os momentos quando houve mudanças nas condições de carga. Além disso, as metodologias resultariam inaplicáveis quando não houver mudanças significativas nas cargas de operação da estrutura.

Devido ao anterior problema, uma solução foi proposta por Yan e Ren (2012) baseado no conceito de função de transmissibilidade escalar com densidade espectral (Power Spectrum Density Transmissibility-PSDT). As PSDTs convergem para a mesma relação de modos de vibração nos pólos do sistema para diferentes referências de saídas. Portanto, fazendo uma combinação de diferentes PSDTs com diferentes referências da saída ao invés de diferentes condições de carga parâmetros modais podem ser identificados. Yan e Ren propuseram usar uma média ponderada entre diferentes funções que surgem de realizar uma inversa da função subtração entre duas PSDTs com diferentes referências da saída.

Sendo assim, neste trabalho procura-se dar uma continuidade aos recentes trabalhos dirigidos a avaliar parâmetros modais com uso da transmissibilidade, mediante a proposição 
de novas alternativas de análise que resultem em melhoras. Dessa forma, na seguinte seção serão consignados os objetivos a serem atingidos com a presente pesquisa.

\subsection{OBJETIVOS.}

\subsubsection{Objetivo geral.}

Desenvolver novas alternativas de identificação modal para estruturas sob excitações em condição de operação baseadas em funções de transmissibilidade.

\subsubsection{Objetivos específicos.}

Os seguintes objetivos específicos são contemplados na consecução do objetivo geral apontado:

- Programar uma ferramenta computacional que possa combinar: análise modal teórica, análise dinâmica e identificação modal sobre modelos estruturais no plano.

- Propor uma metodologia de identificação modal baseada em matrizes de funções de transmissibilidade escalar com densidade espectral.

- Propor uma metodologia de identificação modal baseada em matrizes de transmissibilidade multivariável com diferentes referências da saída.

- Propor uma melhora na metodologia baseada em matrizes de funções de transmissibilidade escalar com densidade espectral.

\subsection{CONTRIBUÇÕES DO TRABALHO}

A seguir apresenta-se o foco e as principais contribuições que contêm cada um dos diferentes capítulos que constituem esta tese.

No Capítulo 2 é apresentada uma introdução aos fundamentos teóricos da análise modal operacional, abordando diferentes aspectos importantes e necessários para o entendimento das metodologias de identificação modal, como são: a teoria básica de vibrações aleatórias, formas de representação do comportamento dinâmico, no domínio do tempo e da frequência, e o conceito de transmissibilidade entre respostas de um sistema linear.

No Capítulo 3 é realizada uma revisão detalhada das diferentes técnicas utilizadas para a estimação de funções de correlação e funções de densidade espectral a partir de 
dados. Ditos parâmetros são variáveis relevantes que devem ser definidas em qualquer método de identificação modal. Igualmente, são descritas diferentes metodologias no domínio da frequência para extrair parâmetros modais do sinal, bem como aquelas metodologias baseadas em transmissibilidade.

No Capítulo 4 apresenta-se uma nova proposta para a identificação de parâmetros modais (frequências naturais e modos de vibração) baseada na aplicação de uma decomposição de valores singulares sobre matrizes de funções de transmissibilidade escalar com densidade espectral. As matrizes de transmissibilidade propostas são singulares quando convergem aos pólos do sistema, portanto os valores singulares convergem para zero e podem ser usados para determinar frequências naturais e modos de vibração. Um exemplo numérico sobre uma viga e uma aplicação sobre dados reais de vibração de uma ponte foram realizados para demostrar a habilidade do método proposto para a identificação de parâmetros modais, sendo comparados com os resultados de outras metodologias.

No Capítulo 5 introduz-se a proposta de se avaliarem funções de transmissibilidade multivariável com diferente referência da saída para identificação de parâmetros modais. A função de transmissibilidade multivariável permite relacionar múltiplas saídas ao invés de só duas como nas funções de transmissibilidade escalar usadas no Capítulo 4. Uma importante propriedade surge a partir das funções de transmissibilidade multivariável, na qual diferentes matrizes multivariáveis com as mesmas saídas relacionadas, mas com diferentes referências da saída convergem para a mesma matriz nos pólos do sistema. Dessa forma, é possível construir uma única matriz a partir de diferentes matrizes multivariáveis que seja singular nos pólos do sistema e aplicar uma pseudo-inversa (Moore-Penrose) modificada para determinar as frequências naturais amortecidas e taxas de amortecimento. O método foi verificado em uma análise numérica de uma viga e com dados reais de vibração de uma ponte.

No Capítulo 6 apresenta-se uma melhora do método apresentado no Capítulo 4. O método proposto no Capítulo 4 é desprovido da habilidade para a identificação de taxas de amortecimento. Portanto, uma melhora é proposta baseada no uso das mesmas matrizes de transmissibilidade propostas no Capítulo 4, mas aplicando sobre elas uma inversa MoorePenrose modificada como proposto no Capítulo 5 para a identificação de parâmetros modais (frequências naturais amortecidas e as taxas de amortecimento). No uso da inversa modificada é preciso estabelecer o número máximo de valores singulares, o qual depende do número de cargas não correlacionadas presente na estrutura. Dessa forma, adicionalmente neste capítulo foi proposto um procedimento de identificação automática do 
número de valores singulares que se deve usar na inversa. Uma análise numérica foi realizada para avaliar o desempenho do método proposto.

No Capítulo 7 apresenta-se o uso das metodologias propostas de identificação modal dos capítulos anteriores, no caso da existência de uma única fonte de excitação. Os métodos propostos têm limitações de identificação para uma condição de uma única fonte de excitação localizada em um ponto, no entanto, neste capítulo foi demostrado que uma única carga sendo deslocada em diferentes pontos da estrutura permite realizar uma identificação modal.

No Capítulo 8 são apresentadas as conclusões obtidas da realização da presente pesquisa e se propõem alguns tópicos que permitam a elaboração de trabalhos futuros.

No Apêndice A é apresentada uma guia introdutória ao uso de uma ferramenta computacional proposta pelo autor desta pesquisa, chamada Myfem Modal 2d, para a análise modal, na qual são descritos de forma geral algumas das diferentes utilidades contidas no programa e se estabelecem diversos tipos de exemplos para a análise modal teórico e de dados de resposta sobre um modelo estrutural. 



\section{CAPÍTULO 2. FUNDAMENTOS ANALITÍCOS DA ANÁLISE MODAL OPERACIONAL.}

Como foi mostrado no capítulo introdutivo o presente trabalho é dedicado ao estudo de métodos de identificação modal, especificamente em análise modal operacional. Assim, antes de apresentar e/ou estudar diferentes metodologias de identificação modal é necessário tratar alguns fundamentos analíticos, nos quais são baseadas estas metodologias.

Neste capítulo são apresentadas: 1) a teoria básica de vibrações aleatórias, que estabelece a relação das densidades espectrais de entrada e de saída de um sistema linear, 2) as diversas formas de representação do comportamento dinâmico, no domínio do tempo, e da frequência para sistemas excitados por sinais aleatórios, como são: sistemas de um só grau de liberdade, sistemas de múltiplos graus de liberdade com amortecimento clássico e amortecimento geral, e 3) o conceito de transmissibilidade entre respostas aleatórias de um sistema linear, analisando a transmissibilidade escalar e a transmissibilidade multivariável entre múltiplos graus de liberdade. Também é analisada uma propriedade importante da transmissibilidade quando elas se aproximam às frequências de ressonância do sistema. Dita propriedade tem uma relação direta com os modos de vibração do sistema. Além disso, ao longo deste capítulo são reveladas e analisadas as principais suposições da análise modal operacional, que são fundamentais na maioria das metodologias de identificação modal que serão estudadas.

Assim, o presente capítulo tem como objetivo criar bases conceituais para o entendimento das metodologias de identificação modal que serão apresentadas em capítulos posteriores.

\subsection{VIBRAÇÕES ALEATÓRIAS}

Os fundamentos do OMA partem da relação da resposta $x(n)$ e a entrada $f(n)$ em um sistema linear e invariante no tempo (Linear Time-Invariant-LTI). A resposta $x(n)$ e a entrada $f(n)$ são estabelecidos como processos aleatórios no sentido amplo estacionário (WideSense Stationarity-WSS), em que a média não depende do tempo e a covariância depende da separação entre amostras, (n2-n1) (Kay, 2006). Portanto, se $x(n)$ e $f(n)$ são processos randômicos WSS e suas covariâncias dependem somente da separação entre amostras 
pode-se definir um tipo de momento conjunto, chamado de sequência de auto-correlação e correlação cruzada para dados discretos, assim.

$$
\begin{aligned}
& R_{x x}(k)=R_{x x}\left(n_{2}-n_{1}\right)=E\left\{x\left(n_{1}\right) x\left(n_{2}\right)\right\}=E\{x(n) x(n+k)\}=\frac{1}{N} \sum_{n=0}^{N-1} x(n) \cdot x(n+k) \\
& R_{f x}(k)=R_{x f}\left(n_{2}-n_{1}\right)=E\left\{x\left(n_{1}\right) f\left(n_{2}\right)\right\}=E\{f(n) x(n+k)\}=\frac{1}{N} \sum_{n=0}^{N-1} f(n) \cdot x(n+k)
\end{aligned}
$$

Em que, $n_{1}=n, n_{2}=n+k, k=n_{2}-n_{1}, R_{x x}(k)$ é a sequência de auto-correlação de $x(n), R_{f x}(k)$ é a correlação cruzada entre $x(n)$ e $f(n), E\{\}$ é o operador da esperança matemática e $N$ é a quantidade de elementos em $x(n)$ e $f(n)$.

As funções de correlação caracterizam-se por ter as seguintes propriedades.

- $\quad$ São positivas para $\mathrm{k}=0$ ou $r_{x}(0)>0$

- $\quad$ São uma sequência par ou $r_{x}(-k)=r_{x}(k)$

- O máximo valor absoluto é em $\mathrm{k}=0$ ou $\left|r_{x}(k)\right| \leq r_{x}(0)$

- A variância do processo randômico é dada por $\sigma_{x}{ }^{2}=r_{x}(0)$

Se o sistema LTI é submetido a múltiplas excitações aleatórias em pontos definidos, $f_{1}(t), f_{2}(t), f_{3}(t) \ldots . . f_{N}(t)$ e é considerada uma resposta do sistema nas posições $x_{1}(t), x_{2}(t), x_{3}(t) \ldots . . x_{N}(t)$, um conjunto de respostas impulsivas que relacionam as saídas com as entradas $h_{x_{1} f_{1}}(t), \ldots ., h_{x_{1} f_{N}}(t), \ldots . ., h_{x_{N} f_{1}}(t), \ldots . ., h_{x_{N} f_{N}}(t)$ podem ser obtidas, considerando condições iniciais de velocidade e deslocamento iguais à zero no sistema. Pode-se expressar uma resposta aleatória do sistema pela soma de diferentes convoluções (Fontul et al., 2004), como.

$$
\begin{aligned}
x_{k}(n)= & h_{x_{k} f_{1}}(n) \times f_{1}(n)+h_{x_{k} f_{2}}(n) \times f_{2}(n), \ldots, h_{x_{k} f_{N}}(m) \times f_{N}(n) \\
& =\sum_{m=-\infty}^{\infty} h_{x_{k} f_{1}}(m) \cdot f_{1}(n-m)+\sum_{m=-\infty}^{\infty} h_{x_{k} f_{2}}(m) \cdot f_{2}(n-m), \ldots, \sum_{m=-\infty}^{\infty} h_{x_{k} f_{N}}(m) \cdot f_{N}(n-m) \\
& =\sum_{s=1}^{N} h_{x_{k} f_{s}}(n) \times f_{s}(n)
\end{aligned}
$$

Em que, $\times$ é o operador da convolução. Então, com o uso das Equações (2-1) e (2-2) podem-se avaliar as correlações $R_{f, x}$ e $R_{x, x}$, o que resulta nas seguintes relações. 


$$
\begin{aligned}
R_{f_{r} x_{j}}(n+k-n) & =E\left\{f_{r}(n) x_{j}(n+k)\right\} \\
= & E\left\{f_{r}(n)\left[\sum_{l=-\infty}^{\infty} h_{x_{j} f_{l}}(l) \cdot f_{1}(n+k-l)+\sum_{l=-\infty}^{\infty} h_{x_{j} f_{2}}(l) \cdot f_{2}(n+k-l), \ldots, \sum_{l=-\infty}^{\infty} h_{x_{j} f_{N}}(l) \cdot f_{N}(n+k-l)\right]\right\} \\
& =\sum_{l=-\infty}^{\infty} h_{x_{j}, f_{1}}(l) \cdot E\left\{f_{r}(n) \cdot f_{1}(n+k-l)\right\}+\sum_{l=-\infty}^{\infty} h_{x_{j} f_{2}}(l) \cdot E\left\{f_{r}(n) \cdot f_{2}(n+k-l)\right\}, \ldots, \sum_{l=-\infty}^{\infty} h_{x_{j} f_{N}}(l) \cdot E\left\{f_{r}(n) \cdot f_{N}(n+k-l)\right\} \\
& =\sum_{l=-\infty}^{\infty} h_{x_{j} f_{1}}(l) \cdot R_{f_{r} f_{1}}(k-l)+\sum_{l=-\infty}^{\infty} h_{x_{j} f_{2}}(l) \cdot R_{f_{r} f_{2}}(k-l), \ldots, \sum_{l=-\infty}^{\infty} h_{x_{j} f_{N}}(l) \cdot R_{f_{r} f_{N}}(k-l)
\end{aligned}
$$

A Equação (2-3) se pode reescrever como um somatório de convoluções, assim.

$$
R_{f_{r} x_{j}}(k)=\sum_{s=1}^{N} R_{f_{r} f_{s}}(k) \times h_{x_{j} f_{s}}(k)
$$

Da mesma forma, obtém-se.

$$
R_{x_{j} f_{r}}(k)=\sum_{s=1}^{N} h_{x_{j} f_{s}}(-k) \times R_{f_{s} f_{r}}(k)
$$

A correlação $R_{x_{k} x_{j}}$ pode ser determinada, como segue.

$$
\begin{aligned}
R_{x_{k} x_{j}}(n+k-n) & =E\left\{x_{k}(n) x_{j}(n+k)\right\} \\
& =E\left\{\left[\sum_{m=-\infty}^{\infty} h_{x_{k} f_{1}}(m) \cdot f_{1}(n-m)+\sum_{m=-\infty}^{\infty} h_{x_{k} f_{2}}(m) \cdot f_{2}(n-m), \ldots, \sum_{m=-\infty}^{\infty} h_{x_{k} f_{N}}(m) \cdot f_{N}(n-m)\right] x_{j}(n+k)\right\} \\
& =\sum_{m=-\infty}^{\infty} h_{x_{k}, f_{1}}(m) \cdot E\left\{f_{1}(n-m) x_{j}(n+k)\right\}+\sum_{m=-\infty}^{\infty} h_{x_{k} f_{2}}(m) \cdot E\left\{f_{2}(n-m) x_{j}(n+k)\right\}, \ldots, \sum_{m=-\infty}^{\infty} h_{x_{k} f_{N}}(m) \cdot E\left\{f_{N}(n-m) x_{j}(n+k)\right\} \\
& =\sum_{m=-\infty}^{\infty} h_{x_{k} f_{1}}(m) \cdot R_{f_{1} x_{j}}(m+k)+\sum_{m=-\infty}^{\infty} h_{x_{k} f_{2}}(m) \cdot R_{f_{2} x_{j}}(m+k), \ldots, \sum_{m=-\infty}^{\infty} h_{x_{k} f_{N}}(m) R_{f_{N} x_{j}}(m+k)
\end{aligned}
$$

Tendo em conta que a correlação se relaciona com a convolução como $E\{x(n) x(n+k)\}=x(-n) \times x(n)$ a equação anterior se pode escrever como.

$$
R_{x_{k} x_{j}}(k)=\sum_{r=1}^{N} h_{x_{k} f_{r}}(-k) \times R_{f_{r} x_{j}}(k)
$$

Da mesma forma.

$$
R_{x_{j} x_{k}}(k)=\sum_{r=1}^{N} R_{x_{j} f_{r}}(k) \times h_{x_{k} f_{r}}(k)
$$

Visando determinar uma relação das correlações da entrada com as correlações das respostas, combinam-se as Equações (2-4) e (2-7), resultando a seguinte formulação.

$$
R_{x_{k} x_{j}}(k)=\sum_{r=1}^{N} \sum_{s=1}^{N} h_{x_{k} f_{r}}(-k) \times h_{x_{j} f_{s}}(k) \times R_{f_{r} f_{s}}(k)
$$


Em que, $R_{x_{k} x_{j}}$ são as funções de correlação da resposta, $R_{f_{r} f_{s}}$ são as funções de correlação da entrada, e $h_{x_{k} f_{r}}, h_{x_{j} f_{s}}$ são as funções de resposta impulsiva do sistema.

\section{Densidade espectral de potência (PSD).}

O valor da energia das frequências contidas dentro de um processo randômico WSS é determinado aplicando a transformada de Fourier. Essa medida é conhecida como a densidade espectral de potência (Power Spectral Density- PSD) ou também como Periodograma. A qual é definida para um processo randômico com um intervalo finito de valores, $n=0,1, \ldots, N-1$, através do estimador da PSD da seguinte forma (Kay, 2006).

$$
S_{x x}(i \omega)=\frac{1}{N}\left|\sum_{n=0}^{N-1} x(n) e^{-i \omega n}\right|^{2}
$$

Outra aproximação para se obtiver o estimador da PSD é baseada no conhecimento da sequência de correlação como definida na relação de Wiener-Khintchine (Kay, 2006).

$$
S_{x_{k} X_{j}}(i \omega)=\sum_{k=-\infty}^{\infty} \hat{R}_{x_{k} X_{j}}(k) e^{-i \omega k}
$$

Com

$$
R_{x_{k} x_{j}}(k)=\frac{1}{N} \sum_{n=0}^{N-1-k} x_{k}(n) x_{j}(n+k) \quad k=0,1, \ldots, N-1
$$

As funções de densidade espectral caracterizam-se por ter as seguintes propriedades.

- PSD não é negativa $0 \leq \hat{S}_{x x}(i \omega) \leq \infty$

- PSD é simétrica com respeito a $\omega=0, \hat{S}_{x x}(-i \omega)=\hat{S}_{x x}(i \omega)$

- A variância é dada por $\sigma_{x x}{ }^{2}=R_{x x}(0)=\int_{-\infty}^{\infty} \hat{S}_{x x}(\omega) d \omega$

- A função de correlação pode ser recuperada da PSD usando a transformada inversa de Fourier.

$$
R_{x x}(k)=\frac{1}{2 \pi} \int_{-\infty}^{\infty} \hat{S}_{x x}(i \omega) e^{i \omega k} d \omega
$$

Retomando as Equações (2-4) e (2-7) e aplicando a transformada de Fourier em ambos os membros das equações e depois combinar elas podem-se avaliar as PSDs da resposta e a entrada de um sistema $\mathrm{LTI}$, como. 


$$
\begin{aligned}
& S_{f_{r} x_{j}}(i \omega)=\sum_{s=1}^{N} H_{x_{j} f_{s}}(i \omega) \cdot S_{f_{r} f_{s}}(i \omega) \\
& S_{x_{k} x_{j}}(i \omega)=\sum_{r=1}^{N} H^{*}{ }_{x_{k} f_{r}}(i \omega) \cdot S_{f_{r} x_{j}}(i \omega) \\
& S_{x_{k} x_{j}}(i \omega)=\sum_{r=1}^{N} \sum_{s=1}^{N} H^{*}{ }_{x_{k} f_{r}}(i \omega) \cdot H_{x_{j} f_{s}}(i \omega) \cdot S_{f_{r} f_{s}}(i \omega)
\end{aligned}
$$

Em que, $S_{x_{k} x_{j}}$ é a densidade espectral da resposta, $S_{f_{r} f_{s}}$ é a densidade espectral da entrada, e $H^{*}{ }_{x_{k}} f_{r}$ ou $H_{x_{j} f_{s}}$ são as funções de transferência, com * representando o complexo conjugado.

Neste ponto é importante mencionar que a maioria dos métodos de identificação modal para OMA supõem que as excitações naturais ou ambientais de entrada ao sistema LTI, contêm uma ampla faixa de frequências de igual contribuição tais que as frequências naturais mais importantes da estrutura podem ser excitadas. Esta suposição traz como consequência que as excitações, $S_{f_{r} f_{s}}$ da Equação (2-16) sejam consideradas como processos randômico do tipo ruído branco.

\section{Ruído branco gaussiano (White Gaussian Noise-WGN)}

Para dois processos randômicos $f_{r}, f_{s}$ WGN diferentes, sendo WSS com média zero, e com idêntica variância $\sigma^{2}$ pode-se definir a correlação, como (Kay, 2006).

$$
R_{f_{r} f_{s}}(k)=E\left\{f_{r}(n) f_{s}(n+k)\right\}=\delta_{f, s} \delta(k) \sigma^{2} \operatorname{Com} \delta(k)=\left\{\begin{array}{ll}
1 & k=0 \\
0 & k \neq 0
\end{array}\right\}, \delta_{f, s}=\left\{\begin{array}{ll}
1 & f=s \\
0 & f \neq s
\end{array}\right\}
$$

Para esses mesmos processos randômicos WGN, define-se o PSD, como.

$$
S_{f_{r} f_{s}}(i \omega)=\sum_{k=-\infty}^{\infty} R_{f_{r} f_{s}}(k) e^{-i \omega k}=\sum_{k=-\infty}^{\infty} \sigma^{2} \delta_{f, s} \delta(k) e^{-i \omega k}=\delta_{f, s} \sigma^{2}
$$

Portanto, se diferentes processos randômico do tipo ruído branco são filtrados por um sistema LTI, obtém-se da Equação (2-16) a seguinte relação.

$$
S_{x_{k} x_{j}}(i \omega)=\sum_{r=1}^{N} \sum_{s=1}^{N} H^{*}{ }_{x_{k} f_{r}}(i \omega) \cdot H_{x_{j} f_{s}}(i \omega) \cdot \delta_{f, s} \sigma^{2}
$$


Também, para um processo randômico tipo WGN a partir da Equação (2-9) a autocorrelação de $x(n)$ se-estabelece, como.

$$
R_{x_{k} x_{j}}(k)=\sum_{r=1}^{N} \sum_{s=1}^{N} h_{x_{k} f_{r}}(-k) \times h_{x_{j} f_{s}}(k) \times \delta_{f, s} \partial(k) \sigma^{2}
$$

Uma vez que $h_{x_{j} f_{s}}(k) \times \partial(k)=h_{x_{j} f_{s}}(k)$

$$
R_{x_{k} x_{j}}(k)=\sum_{r=1}^{N} \sum_{s=1}^{N} h_{x_{k} f_{r}}(-k) \times h_{x_{j} f_{s}}(k) \times \delta_{f, s} \sigma^{2}
$$

E voltando a uma representação de somatórios de convoluçao, resulta.

$$
R_{x_{k} x_{j}}(k)=\sigma^{2}\left[\sum_{m=-\infty}^{\infty} h_{x_{k} f_{1}}(m) \cdot h_{x_{j} f_{1}}(m+k)+. .+\sum_{m=-\infty}^{\infty} h_{x_{k} f_{1}}(m) \cdot h_{x_{j} f_{N}}(m+k)+\ldots+\sum_{m=-\infty}^{\infty} h_{x_{k} f_{N}}(m) h_{x_{j} f_{N}}(m+k)\right]
$$

Então, a partir de considerar umas entradas ao sistema LTI como processos randômicos tipo ruído branco podem ser levantadas duas questões.

1. A densidade espectral obtida unicamente da resposta do sistema permite determinar diretamente suas propriedades dinâmicas, devido ao fato de ser calculada como um produto entre as funções de transferência e uma constante (ver Equação (2-19)).

2. A transformada inversa de Fourier (Inverse Fast Fourier Transform-IFFT) da densidade espectral da resposta é igual às funções de correlação da resposta, as quais são proporcionais às funções de resposta impulsiva do sistema original (ver Equação (2-22)). Assim, as funções de correlação da resposta podem ser usadas como funções de resposta impulsiva no domínio do tempo para estimação de parâmetros modais. Uma demonstração desse fato pode ser encontrada em James, Carne et al., (1993).

\subsection{RESPOSTA DE UM SISTEMA LTI-SDF}

A resposta de um sistema LTI de um só grau de liberdade (Single Degree of Freedom-SDF) é descrito por uma equação dinâmica de segundo grau, considerando amortecimento viscoso proporcional à velocidade, da seguinte forma.

$$
\ddot{x}(t)+2 \xi \omega_{0} \dot{x}(t)+\omega_{0}{ }^{x} x(t)=f(t) / m \quad \text { com } \omega_{0}=\sqrt{k / m}, \quad \xi=\frac{c}{2 m \omega_{0}}=\frac{c}{c_{c}}
$$

Em que, $x(t)$ é a resposta do sistema no tempo, $f(t)$ é a excitação, $\omega_{0}$ é a frequência natural do sistema, $k$ a constante de rigidez, $m$ a massa do sistema, $c$ a constante de amortecimento e $\xi$ é a porcentagem de amortecimento com respeito ao amortecimento 
crítico $c_{c}$. Aplicando a transformada de Laplace na Equação (2-21), com condições iniciais nulas e considerando a variável de Laplace como um número complexo $s=i \omega$, pode-se obter uma relação entre a entrada e saída no sistema, assim.

$$
X(i \omega)=H_{x f}(i \omega) F(i \omega)=\frac{1}{m\left(\omega_{0}^{2}-\omega^{2}+2 i \xi \omega_{0} \omega\right)} F(i \omega)
$$

Em que, $H_{x f}$ é a função de transferência harmônica para um sistema SDF.

A partir de usar o denominador da função de transferência igual à zero, $\omega_{0}{ }^{2}-(i \omega)^{2}+2 i \xi \omega_{0} \omega=0$ pode-se avaliar a frequência de excitação para que o sistema entre em ressonância (pólo do sistema), resolvendo o polinômio de grau dois para i $\omega=\lambda$ e considerando um sistema sub-amortecido, resulta.

$\lambda=-\xi \omega_{0} \pm i \omega_{0} \sqrt{1-\xi^{2}}$ se $\xi<1$

Da Equação (2-25) pode-se extrair informação com respeito: à frequência amortecida, não amortecida e a porcentagem de amortecimento, assim.

$$
\omega_{d}=\operatorname{imag}(\lambda), \omega_{o}=|\lambda|, \xi=-\operatorname{real}(\lambda) /|\lambda|
$$

Além disso, uma situação de interesse na Equação (2-24) é a definição da resposta do sistema a uma excitação devido a um impulso ou função Delta Dirac, como $f(t)=\delta(t)$. Aqui a transformada de Laplace do Delta Dirac é exatamente igual $L\{\delta(t)\}=1$, portanto, a resposta impulsiva no domínio da frequência fica exatamente igual à função de transferência. Então, para avaliar a resposta impulsiva no domínio do tempo é só aplicar a transformada inversa de Fourier à função de transferência do sistema, e assim obter a seguinte representação.

$$
h_{x f}(t)=F^{-1}\left\{H_{x f}(i \omega)\right\}=\frac{1}{2 \pi} \int_{-\infty}^{\infty}\left(m\left(\omega_{0}^{2}-\omega^{2}+2 i \xi \omega_{0} \omega\right)\right)^{-1} e^{i \omega t} d \omega
$$

No caso de vibrações aleatórias, sendo $f(t)$ um processo randômico WSS, pode-se usar a Equação (2-16) para representar a densidade espectral de potência para um sistema SDF, como.

$$
S_{x x}(i \omega)=H^{*}{ }_{x f}(i \omega) \cdot H_{x f}(i \omega) \cdot S_{f f}(i \omega)=\left|H_{x f}(\omega)\right|^{2} \cdot S_{f f}(i \omega)=\frac{S_{f f}(i \omega)}{m^{2}\left[\left(\omega_{0}{ }^{2}-\omega^{2}\right)^{2}+\left(2 \xi \omega_{0} \omega\right)^{2}\right]}
$$


Como foi determinado na Equação (2-13) é possível avaliar a função de autocorrelação aplicando a transformada inversa de Fourier ao espectro de resposta, desse modo pode-se determinar a função de correlação na Equação (2-28), assim.

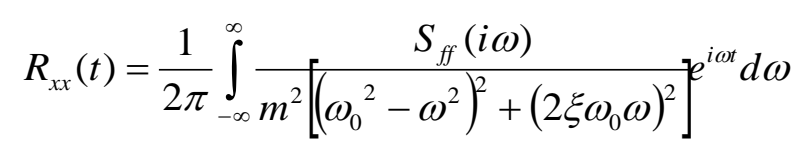

Além disso, pode-se analisar na Equação (2-28) uma justificativa para estabelecer uma aproximação de $f(t)$ como um processo randômico Gaussiano tipo WGN de espectro plano em OMA, no entanto, existem algumas limitações. A razão é devida principalmente ao produto de $S_{f f}(\omega)$ e $\left|H_{x f}(\omega)\right|^{2}$. Nesse produto a parcela mais significativa da multiplicação chega a ser perto da frequência de ressonância $\left(\omega \approx \pm \omega_{0}\right)$. Portanto, a precisão dessa aproximação depende principalmente da largura da banda da ressonância em $\omega \approx \pm \omega_{0}$, a qual é influenciada principalmente pela porcentagem de amortecimento $\xi$.

Na Figura 2-1 e na Figura 2-2 mostra-se uma situação em que considerando $S_{f f}(\omega)$ constante como ruído branco, quando realmente existe uma excitação de tipo ruído colorido com uma frequência definida de $\omega_{f f}=0.8 \mathrm{rad} / \mathrm{s}$, não é adequada para uma porcentagem de amortecimento do sistema de $\xi=0.15$. Porém, parece ser aceitável para uma porcentagem de $\xi=0.05$. O ruído colorido estabelecido em $S_{f f}(\omega)$ foi modelado como um sistema de um grau de liberdade com um amortecimento de 0.4.

Nesse sentido, é preciso estabelecer que uma aproximação do espectro da excitação como do ruído branco pode em algumas ocasiões conduzir a uma avaliação errônea das frequências naturais amortecidas e das taxas de amortecimento no espectro da saída. Igualmente, uma excitação com uma frequência bem definida geraria um pico de frequência no espectro da saída que realmente não faz parte do sistema analisado e que pode ser confundido como pertencentes a ele, como se mostra na Figura 2-3, na que foi modelado um espectro de entrada como um SDF com uma frequência de $\omega_{f f}=0.8 \mathrm{rad} / \mathrm{s}$ e amortecimento de 0.1 . 


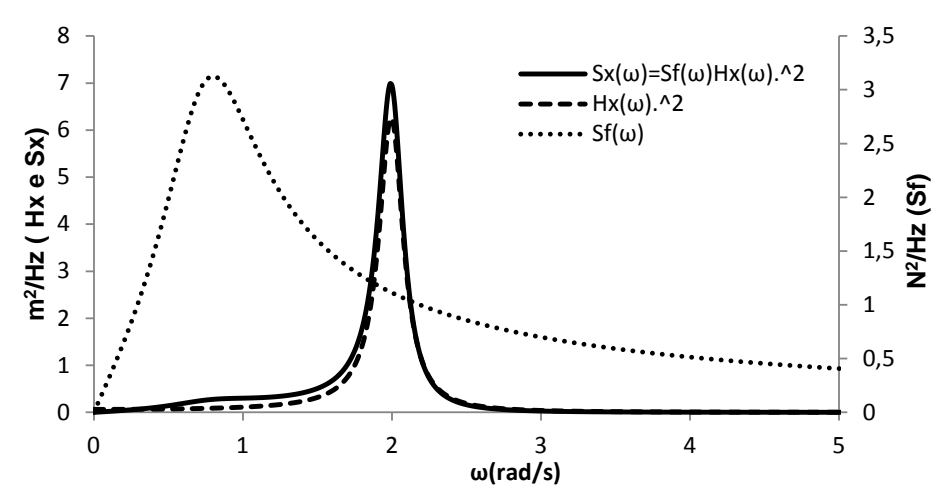

Figura 2-1 Densidade espectral da resposta, $\omega_{0}=2 \mathrm{rad} / \mathrm{s}, \xi=0.05$, adequada aproximação como um ruído branco.

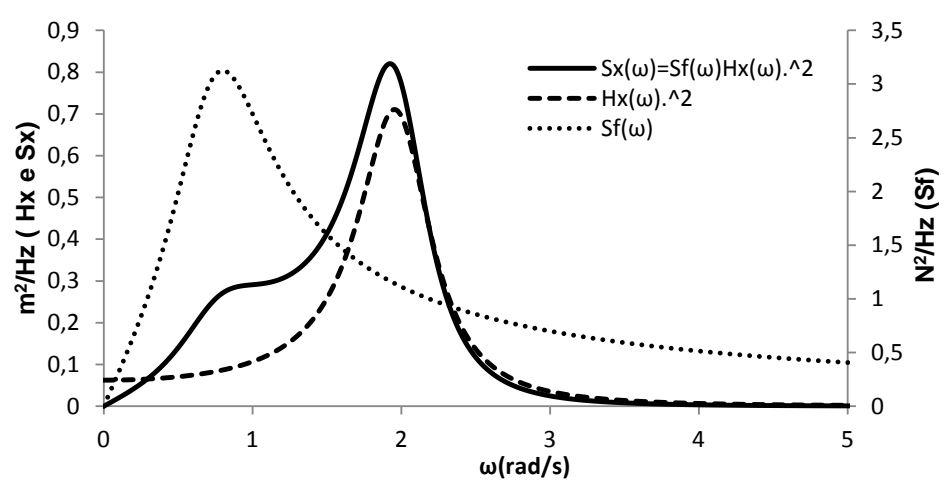

Figura 2-2 Densidade espectral da resposta, $\omega_{0}=2 \mathrm{rad} / \mathrm{s}, \xi=0.15$, inadequada aproximação como um ruído branco.

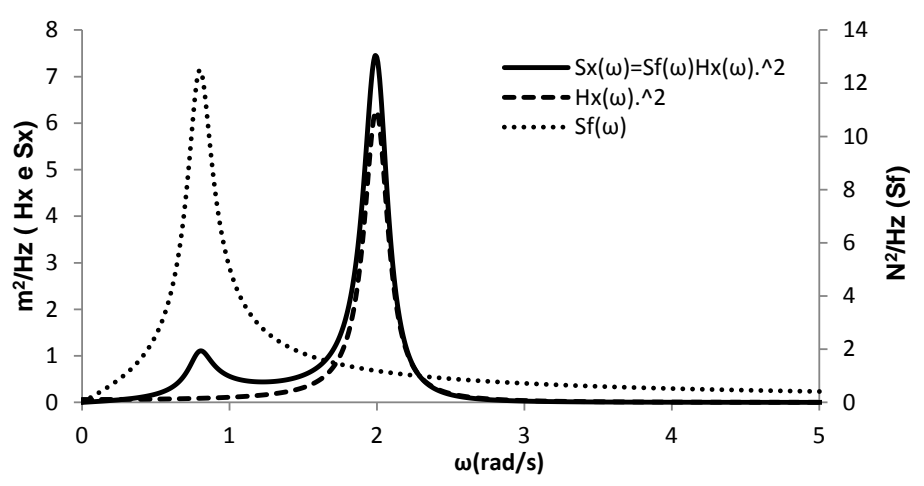

Figura 2-3 Densidade espectral da resposta, $\omega_{0}=2 \mathrm{rad} / \mathrm{s}, \xi=0.15$, inclusão da frequência de excitação no espectro da saída.

Pela anterior análise uma opção mais realista é estabelecer a excitação como um ruído colorido. Nesse caso é assumido que as entradas não observadas ao sistema são um 
ruído branco que passa através de outro sistema LTI, chamado de cor do ruído (Reynders, 2012) (ver Figura 2-4).

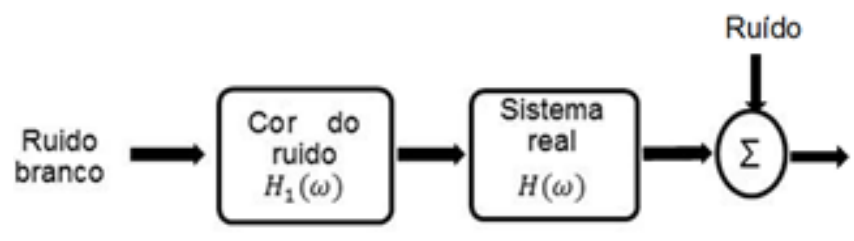

Figura 2-4 Diagrama do sistema a identificar quando a excitação é ruído colorido.

Assim, o novo sistema LTI $H_{1}(\omega)$ passa a ser parte do sistema a identificar. Nesse caso, o sistema $H_{1}(\omega)$ poderia ser separado do sistema real sempre que existir algum tipo de conhecimento a priori dele, por exemplo, o modelo do sistema $H_{1}(\omega)$ e as variáveis que ele contém (Reynders, 2012). Além disso, é conhecido que os modos dos sistemas estruturais civis são de baixo amortecimento e normais entre eles, então os modos altamente amortecidos ou não normais podem ser assumidos como parte do sistema $H_{1}(\omega)$

Desse modo a partir do anterior conceito e usando a Equação (2-28) pode-se representar a resposta para uma excitação com ruído colorido, da seguinte forma.

$$
S_{x x}(i \omega)=\sigma_{f}^{2}\left|H_{1}(i \omega)\right|^{2}\left|H_{x f}(i \omega)\right|^{2}
$$

\subsection{RESPOSTA DE UM SISTEMA LTI-MDF PARA O AMORTECIMENTO PROPORCIONAL.}

No caso de sistemas de múltiplos graus de liberdade (Multiple Degree of FreedomMDF), no qual podem existir múltiplas entradas ou fontes de excitação e múltiplas saídas ou respostas. Uma simples generalização da Equação (2-23) pode ser usada para representar o sistema MDF, como.

$$
[M]\{\ddot{x}(t)\}+[C]\{\dot{x}(t)\}+[K]\{x(t)\}=\{f(t)\}
$$

Na qual $[M],[C]$ e $[K]$ são as matrizes de massa, amortecimento e rigidez, respectivamente, de dimensões $N \times N$, sendo $N$ os graus de liberdade do sistema. A localização e direções das componentes do vetor de excitação $\{f(t)\}$ são os mesmos 
daqueles do vetor de resposta $\{x(t)\}$. Pelo teorema de reciprocidade de Maxwell-Betti de resistência de materiais as matrizes $[M]$ e $[K]$ sempre serão matrizes simétricas, para não gerar negatividade nas energias cinéticas $\frac{1}{2}\{\dot{x}(t)\}[M]\{\dot{x}(t)\}^{T}$ e potenciais $\frac{1}{2}\{x(t)\}[K]\{x(t)\}^{T}$ do sistema, igualmente os auto-valores de $[M]$ e $[K]$ serão reais e positivos (maiores ou iguais a zero), portanto, as matrizes $[M]$ e $[K]$ são definidas positivas. Então, para o caso das matrizes simétricas e positivas $[M]$ e $[K]$ elas têm uma propriedade importante. O problema de auto-vetores dado por $[M]^{-1}[K] \Phi=\Phi \lambda$, resulta em uma matriz de autovetores $\Phi$ que sempre será ortogonal, portanto, linearmente independentes. Esse fato implica que as matrizes $[M]$ e $[K]$ sejam sempre diagonalizáveis. Assim é possível escrever a Equação (2-25), como.

$\left.\left.[\Phi]^{T}[M][\Phi]\right\} \ddot{z}(t)\right\}+[\Phi]^{T}[C][\Phi]\{\dot{z}(t)\}+[\Phi]^{T}[K][\Phi]\{z(t)\}=[\Phi]^{T}\{f(t)\}$

$\mathrm{Ou}$

$[\hat{M}]\{\ddot{z}(t)\}+[\hat{C}]\{\dot{z}(t)\}+[\hat{K}]\} z(t)\}=[\Phi]^{T}\{f(t)\}$

$\left.\operatorname{Com}\{x(t)\}=[\Phi]\{z(t)\},[\hat{M}]=[\Phi]^{T}[M][\Phi], \mid \hat{C}\right]=[\Phi]^{T}[C][\Phi],[\hat{K}]=[\Phi]^{T}[K][\Phi]$

Uma particular formulação e de grande utilidade para a Equação (2-32) é quando o termo $[\Phi]^{T}[C][\Phi]$ é também diagonalizável a partir dos auto-vetores de $[M]$ e $[K]$, a qual é conhecida como a situação de modos normais clássicos ou também amortecimento clássico com modos não acoplados. Uma condição geral para a existência de modos não acoplados é apresentada em Caughey (1960) como $[K][M]^{-1}[C]=[C][M]^{-1}[K]$. No mesmo trabalho de Caughey (1960) apresenta-se também uma condição suficiente, mas não necessária para existência de modos não acoplados, que é a chamada de condição de Rayleigh, dada por $[C]=a_{1}[M]+a_{2}[K]$ a qual é uma condição especial da condição geral. Também, em Caughey (1960) mostra-se outra condição suficiente e não necessária conhecida como condição generalizada de Rayleigh, dada assim. 


$$
[C]=[M] \sum_{j=1}^{N-1} a_{j}\left([M]^{-1}[K]\right)^{j}
$$

Então, pode-se demostrar que se o limite superior da somatória na Equação (2-33) tende a $N$-1 a Equação (2-33) é equivalente à condição geral mostrada anteriormente. Uma das razões para o uso das anteriores aproximações para a matriz de amortecimento é o limitado conhecimento acerca da forma exata da matriz de amortecimento, principalmente pelo pobre entendimento do fenômeno de dissipação de energia nas estruturas, o qual é tipicamente não linear. Por isso, é selecionada uma forma simplificada de análises, tal como o amortecimento clássico com modos não acoplados para modelar uma grande variedade de sistemas físicos dinâmicos. Então, a partir do conhecimento dos $\lambda_{j}$ da análise de autovalores de $[M]$ e $[K]$ é possível avaliar uma matriz de amortecimento clássica por alguns dos métodos expostos anteriormente. Essa mesma matriz de amortecimento clássica pode ser diagonalizada usando os mesmos auto-vetores de $[M]$ e $[K]$, assim $[\hat{M}]^{-1}[\hat{C}]=\left[{ }^{\prime} 2 \xi_{j} \omega_{j_{\curlywedge}}\right]$. Nesse sentido, pode-se avaliar a Equação (2-32) a uma situação de modos não acoplados, que é essencialmente a mesma equação para sistemas SDF que foi considerada previamente, assim.

$\ddot{z}_{j}(t)+2 \xi_{j} \omega_{j} \dot{z}_{j}(t)+\omega_{j}{ }^{2} z_{j}(t)=\frac{1}{\hat{M}_{j j}} \sum_{l=1}^{N} \Phi_{l j} f_{l}(t) \operatorname{com} j=1,2 \ldots N$

A Equação (2-34) é um resultado importante para a análise modal, já que se reduz um sistema de MDF para um conjunto de sistemas SDF não acoplados. Assim mesmo, pode-se observar que o comportamento dinâmico total de um sistema MDF vem dado pela somatória das contribuições de cada um dos modos de vibração do sistema, assim.

$x_{j}(t)=\sum_{l=1}^{n} \Phi_{j l} z_{l}(t)$

Do mesmo modo que para sistemas SDF pode-se avaliar a função de transferências para sistemas MDF, aplicando a transformada de Laplace na Equação (2-34) e considerando a variável de Laplace como um número complexo $s=i \omega$, obtém-se assim uma relação entre a entrada e a saída para MDF no domínio da frequência, para a resposta modal $Z_{l}(\omega)$, considerando só uma entrada ao sistema, chamada de $F_{r}$, assim.

$$
Z_{l}(i \omega)=[\hat{H}(i \omega)]_{z_{l} f_{r}} F_{r}(i \omega)=\frac{\Phi_{r l}}{\hat{M}_{l l}\left(\omega_{l}{ }^{2}-\omega^{2}+2 i \xi_{l} \omega_{l} \omega\right)} F_{r}(i \omega)
$$


Então, pode-se usar a Equação (2-35) para encontrar a resposta total $X_{k}(t)$ e avaliar a função de transferência $[H(\omega)]$, assim.

$$
\left.X_{k}(i \omega)=[\Phi]_{k l} \mid \hat{H}(i \omega)\right]_{z_{l} f_{r}} F_{r}(i \omega)=[H(\omega)]_{x_{k} f_{r}} F_{r}(i \omega)
$$

$\mathrm{Ou}$

$$
X_{k}(i \omega)=[H(i \omega)]_{x_{k} f_{r}} F_{r}(i \omega)=\sum_{l=1}^{N} \frac{[\Phi]_{k l}[\Phi]_{r l}}{\hat{M}_{l l}\left(\omega_{l}^{2}-\omega^{2}+2 i \xi_{l} \omega_{l} \omega\right)} F_{r}(i \omega)
$$

É possível derivar a função de transferência $[H(\omega)]_{x_{k} f_{r}}$ sem o uso da equação de modos não acoplados, aplicando diretamente a transformada de Laplace na Equação (2-31), resultando.

$$
X_{k}(i \omega)=[H(i \omega)]_{x_{k} f_{r}}\left\{F_{r}(i \omega)\right\}=\frac{1}{[K]-\omega^{2}[M]+i \omega[C]}\left\{F_{r}(i \omega)\right\}
$$

Uma diferença notável entre as Equações (2-37) e (2-38) para avaliar as funções de transferência para MDF é que a Equação (2-38) não precisa da aproximação de um amortecimento clássico com modos não acoplados, assim pode-se avaliar a funções de transferência para qualquer matriz $[C]$.

Uma situação importante do mesmo modo que foi analisado para sistemas SDF é quando a excitação em um grau de liberdade $\boldsymbol{k}$ do sistema é do tipo delta de Dirac $F(t)=\delta(t)$, com a transformada de Laplace igual a $L\{\partial(t)\}=1$. A partir dessa situação de excitação pode-se determinar a matriz de funções de resposta impulsiva do sistema, aplicando a transformada inversa de Fourier diretamente na função de transferência da Equação (2-38) ou (2-37). Mostra-se para a Equação (2-37), como.

$$
[h(t)]_{x_{k} f_{r}}=F^{-1}\left[[H(i \omega)]_{x_{k} f_{r}}\right]=\frac{1}{2 \pi} \int_{-\infty}^{\infty} \sum_{l=1}^{n} \frac{[\Phi]_{k l}[\Phi]_{r l}}{\hat{M}_{l l}\left(\omega_{l}^{2}-\omega^{2}+2 i \xi_{l} \omega_{l} \omega\right)} e^{i \omega t} d \omega
$$

No caso de vibrações aleatórias, em que $F(t)$ é um processo randômico WSS, também é possível usar a Equação (2-16) para representar a densidade espectral de potência para um sistema MDF, como. 


$$
\begin{aligned}
& {[S(i \omega)]_{x_{k x_{j}}}=\sum_{r=1}^{N} \sum_{s=1}^{N} H^{*}{x_{k} f_{r}}_{r}(i \omega) \cdot H_{x_{j} f_{s}}(i \omega) \cdot S_{f_{r} f_{s}}(i \omega)} \\
& {[S(i \omega)]_{x_{k} x_{j}}=\sum_{r=1}^{N} \sum_{s=1}^{N}\left(\left(\sum_{l=1}^{n} \frac{[\Phi]_{k l}[\Phi]_{r l}}{\hat{M}_{l l}\left(\omega_{l}{ }^{2}-\omega^{2}+2 i \xi_{l} \omega_{l} \omega\right)}\right)^{*}\left(\sum_{l=1}^{n} \frac{[\Phi]_{j l}[\Phi]_{s l}}{\hat{M}_{l l}\left(\omega_{l}{ }^{2}-\omega^{2}+2 i \xi_{l} \omega_{l} \omega\right)}\right) S_{f_{r} f_{s}}(i \omega)\right)}
\end{aligned}
$$

Da mesma forma, pode-se avaliar a matriz de funções de correlação aplicando o conceito da Equação (2-13), obtendo a seguinte representação.

$$
[R(t)]_{x_{x} x_{j}}=\frac{1}{2 \pi} \int_{-\infty}^{\infty}\left(\left(\sum_{l=1}^{n} \frac{[\Phi]_{k l}[\Phi]_{l l}}{\hat{M}_{l l}\left(\omega_{l}^{2}-\omega^{2}+2 i \xi_{l} \omega_{l} \omega\right)}\right)^{*}\left(\sum_{l=1}^{n} \frac{[\Phi]_{j l}[\Phi]_{s l}}{\hat{M}_{l l}\left(\omega_{l}{ }^{2}-\omega^{2}+2 i \xi_{l} \omega_{l} \omega\right)}\right) S_{f_{f r_{s}}}(i \omega)\right) e^{i \omega t} d \omega
$$

Em geral na análise modal experimental para o caso de sistemas de múltiplos graus de liberdade, é necessário além de ter uma entrada com um conteúdo em frequência que esteja na faixa de frequências do interesse do sistema, essa mesma entrada deve estar adequadamente aplicada ou distribuída no sistema. Uma inadequada distribuição das forças de entrada pode conduzir em não excitar suficientemente alguns modos naturais, devido principalmente à existência de pontos com amplitudes zeros nos modos de vibração. Portanto, na análise modal operacional, outra suposição além de que as forças de entrada devem excitar as frequências naturais da estrutura, como pode ser uma excitação do tipo ruído branco, também é suposto que a entrada ao sistema é adequadamente distribuída.
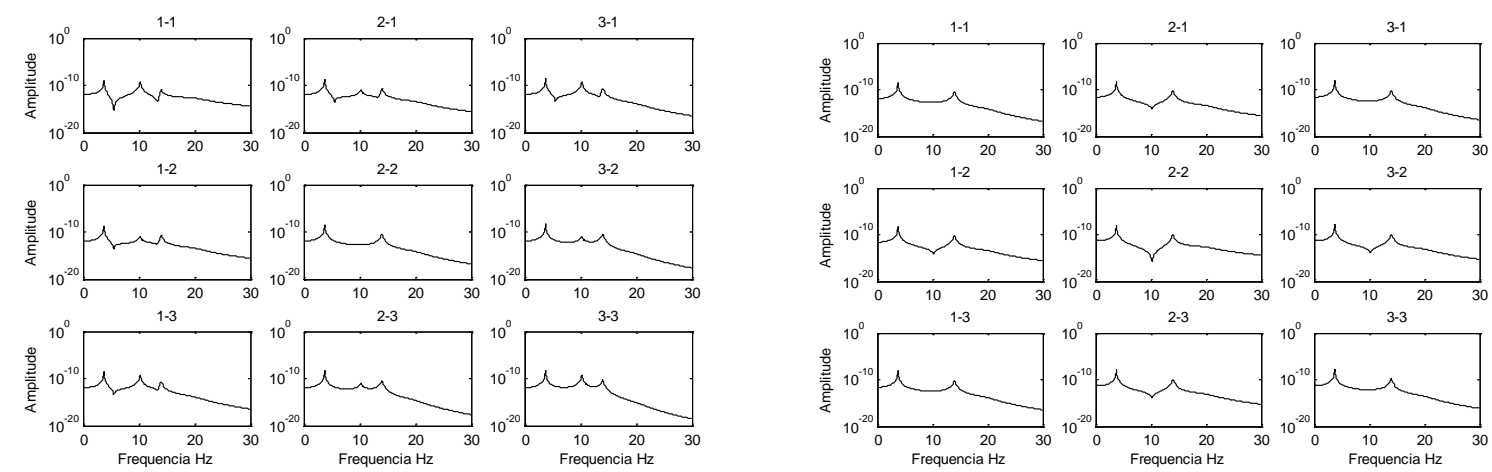

$$
\left[S_{f_{r} f_{s}}(\omega)\right]=\left[\begin{array}{ccc}
1 & 0 & 0 \\
0 & 0 & 0 \\
0 & 0 & 0
\end{array}\right]
$$

$$
\left[S_{f_{r} f_{s}}(\omega)\right]=\left[\begin{array}{lll}
0 & 0 & 0 \\
0 & 1 & 0 \\
0 & 0 & 0
\end{array}\right]
$$

a)

\section{b)}

Figura 2-5 Matriz de densidade espectral de resposta $[S(i \omega)]_{x_{k} x_{j}}$ de um sistema de três graus de liberdade com uma distribuição de excitação de ruído branco aplicado a) no segundo grau de liberdade e b) no primeiro grau de liberdade.

Como exemplo do exposto usou-se a Equação (2-40) para avaliar a matriz de densidade espectral da resposta $[S(i \omega)]_{x_{k} x_{j}}$ a partir de estabelecer dois tipos de matriz 
de densidade espectral de entrada, $[S(i \omega)]_{f_{r} f_{s}}$, de ruído branco (densidade espectral constante) com uma distribuição diferente, uma com a excitação aplicada no segundo grau de liberdade e a outra no primeiro grau de liberdade. A resposta foi avaliada em um sistema de três graus de liberdade, o qual tem para o segundo modo natural uma amplitude modal zero no segundo grau de liberdade. Assim, é mostrada na Figura 2-5 uma excitação aplicada no segundo grau de liberdade, a qual pode excitar só dois modos naturais do sistema (Figura 2-5 b), pelo contrário, uma excitação aplicada no primeiro grau de liberdade pode excitar todos os modos naturais (Figura 2-5 a).

\subsection{RESPOSTA DE UM SISTEMA LTI-MDF PARA O AMORTECIMENTO GERAL.}

Voltando à Equação (2-38), pode-se notar a possibilidade de avaliar as funções de transferência para qualquer tipo de matriz $[C]$, porém, essa expressão não é particularmente conveniente para as aplicações numéricas, devido a que se precisa avaliar uma matriz inversa complexa para cada frequência $\omega$. Portanto, uma forma conveniente seria estabelecer uma expressão similar de sistemas SDF não acoplados como foi feita para sistemas MDF com amortecimento proporcional. Nesse sentido, é necessário reformular a equação dinâmica (2-31) na forma de espaço de estado para alcançar o objetivo.

$\mathrm{Na}$ formulação de espaço de estado transforma-se um sistema de $\mathrm{N}$ equações diferenciais de segundo ordem (Equação (2-31)) para um sistema de $2 \mathrm{~N}$ equações diferenciais de primeira ordem com a introdução de um vetor de estado, assim.

$$
q(t)=\left\{\begin{array}{c}
x(t) \\
\dot{x}(t)
\end{array}\right\}
$$

Podemos reescrever a Equação (2-31) da seguinte forma.

$\left.\left.[[C][M]]_{N \times 2 N}\left\{\begin{array}{l}\dot{x}(t) \\ \ddot{x}(t)\end{array}\right\}_{2 N \times 1}+[[K][0]]\right\} \begin{array}{l}x(t) \\ \dot{x}(t)\end{array}\right\}=\{F(t)\}$

$\mathrm{Ou}$

$[[C] \quad[M]]_{N \times 2 N}\{\dot{q}(t)\}_{2 N \times 1}+[[K] \quad[0]\{q(t)\}=\{F(t)\}$ 
Porém, nessa forma tem-se $\mathrm{N}$ equações com $2 \mathrm{~N}$ variáveis desconhecidas tendo que se adicionar uma equação identidade para igualar o número de variáveis com o número de equações, assim.

$[[M][0]]_{N \times 2 N}\{\dot{q}(t)\}_{2 N \times 1}+[0]-[M]\{q(t)\}=\{F(t)\}$

As quais podem ser combinadas para configurar o conjunto de $2 \mathrm{~N}$ equações.

$\left[\begin{array}{cc}{[C]} & {[M]} \\ {[M]} & {[0]}\end{array}\right]_{2 N \times 2 N}\{\dot{q}(t)\}_{2 N \times 1}+\left[\begin{array}{cc}{[K]} & {[0]} \\ {[0]} & -[M]\end{array}\right]\{q(t)\}=\left\{\begin{array}{c}F(t) \\ \{0\}\end{array}\right\}$

E simplificada da forma.

$\left[A_{s}\right]\{\dot{q}(t)\}+\left[B_{s}\right]\{q(t)\}=\{W(t)\} \operatorname{com}\{W(t)\}=\left[\begin{array}{c}{[B]} \\ {[0]}\end{array}\right]\{F(t)\}$

Aqui o vetor de excitação $F(t)$ é modificado pela introdução de uma matriz $B$, construída por zeros e uns que especifica a localização das entradas. A Equação (2-46) para o caso da solução homogênea representa o problema típico de valores e vetores próprios em que se podem avaliar $2 \mathrm{~N}$ frequências naturais complexas e $2 \mathrm{~N}$ vetores próprios complexos, os quais juntos satisfazem a equação.

$$
\left[A_{s}\right]\left\{\Psi_{j}\right\} \lambda_{j}+\left[B_{s}\right]\left\{\Psi_{j}\right\}=0 \text { com } j=1,2 \ldots .2 N
$$

Em que, $\left\{\Psi_{r}\right\}$ é do tamanho $2 N \times 1$ com $\left\{\Psi_{j=1: N}\right\}$ igual aos vetores próprios complexos e $\left\{\Psi_{j=N+1: 2 N}\right\}$ são os pares conjugadas, $\lambda_{j}$ é um escalar com $\lambda_{j=1: N}$ igual aos valores próprios complexos e $\lambda_{r=N+1: 2 N}$ são seus pares conjugadas.

Do mesmo modo que as matrizes de rigidez e massa, as matrizes $\left[A_{s}\right]$ e $\left[B_{s}\right]$ podem ser diagonalizadas, obtendo as seguintes relações.

$$
\begin{aligned}
& {[\Psi]^{T}\left[A_{s} \rrbracket \Psi\right]=\left[\hat{a}_{j}\right]} \\
& {[\Psi]^{T}\left[B_{s}\right][\Psi]=\left\lfloor\hat{b}_{j}\right\rfloor}
\end{aligned}
$$

Substituindo as Equações (2-48) em (2-47) pode-se obter a seguinte relação modal.

$\lambda_{j}=\hat{a}_{j} / \hat{b}_{j} \operatorname{com} \quad j=1,2 \ldots .2 N$

É necessário considerar que $\lambda_{r}$ responde à mesma formulação feita para sistemas SDF na Equação (2-25), na que se podem avaliar a partir do conhecimento de $\lambda_{r}$ as 
frequências amortecidas, não amortecidas e as taxas de amortecimento com as Equações (2-26).

Do mesmo modo, a partir das propriedades de ortogonalidade das matrizes $\left[A_{s}\right] \mathrm{e}$ $\left[B_{s}\right]$ pode-se determinar uma equação de modos não acoplados para a equação dinâmica em espaço de estado (Equação (2-46)), como segue.

$\hat{a}_{j}\left\{\dot{z}_{j}(t)\right\}+\hat{b}_{j}\left\{z_{j}(t)\right\}_{j}=\sum_{l=1}^{2 N} \Psi_{l j}\left\{W_{l}(t)\right\}$ com $\{q(t)\}=[\Psi]\{z(t)\}$

$\mathrm{Ou}$

$$
\{\dot{z}(t)\}_{j}+\lambda_{j}\{z(t)\}_{j}=\frac{1}{a_{j}} \sum_{l=1}^{2 N} \Psi_{l j}\left\{W_{l}(t)\right\}
$$

Aplicando a transformada de Laplace na Equação (2-50) e considerando a variável de Laplace como um número complexo $s=i \omega$, pode-se obter uma relação entre a entrada e a saída da resposta total para avaliar a função de transferência.

$$
q(i \omega)=[H(i \omega)]_{q w} W(i \omega)=\frac{[\Psi][\Psi]^{T} W(i \omega)}{a_{j}\left(i \omega-\lambda_{j}\right)}
$$

$\mathrm{Ou}$

$$
\left\{q_{k}(i \omega)\right\}=[H(i \omega)]_{q_{k} \omega_{r}}\left\{W_{r}(i \omega)\right\}=\sum_{l=1}^{2 N} \frac{\Psi_{k l} \Psi_{r l} W_{r}(i \omega)}{a_{l}\left(i \omega-\lambda_{l}\right)}
$$

No entanto, devido a que os valores e vetores próprios ocorrem em pares de complexos conjugados, a Equação (2-51) pode ser reescritas, como (Ewins, 2000).

$$
\left\{q_{k}(i \omega)\right\}=[H(i \omega)]_{q_{k} w_{r}}\left\{W_{r}(i \omega)\right\}=\sum_{l=1}^{N}\left(\frac{\Psi_{k l} \Psi_{r l} W_{r}(i \omega)}{a_{l}\left(i \omega-\lambda_{l}\right)}+\frac{\Psi_{k l}^{*} \Psi_{r l}{ }^{*} W_{r}(i \omega)}{a_{l}^{*}\left(i \omega-\lambda_{l}^{*}\right)}\right)
$$

Para vibrações aleatórias, em que $W(t)$ é um processo randômico WSS, representase a densidade espectral de potência para um sistema MDF com amortecimento viscoso geral, como.

$$
[S(i \omega)]_{x_{k} \chi_{j}}=\sum_{r=1}^{N} \sum_{s=1}^{N}\left(\sum_{l=1}^{N}\left(\frac{\Psi_{k l}{ }^{*} \Psi_{r l}{ }^{*}}{a^{*}{ }_{l}\left(-i \omega-\lambda^{*}\right)}+\frac{\Psi_{l l} \Psi_{r l}}{a_{l}\left(-i \omega-\lambda_{l}\right)}\right) \sum_{l=1}^{N}\left(\frac{\Psi_{j l} \Psi_{s l}}{a_{l}\left(i \omega-\lambda_{l}\right)}+\frac{\Psi_{j l}{ }^{*} \Psi_{s l}{ }^{*}}{a_{l}{ }^{*}\left(i \omega-\lambda_{l}{ }^{*}\right)}\right) S_{f_{r} f_{s}}(i \omega)\right)
$$




\subsection{FUNÇÕES DE TRANSMISSIBILIDADE ESCALAR E MULTIVARIÁVEL}

A transmissibilidade escalar se define como a relação de duas respostas, assumindo que existe uma força de entrada localizada em um grau de liberdade $k$. Portanto, a transmissibilidade se reduz à seguinte formulação.

$$
T^{k}{ }_{x_{o} x_{j}}(i \omega)=\frac{X_{o}(i \omega)}{X_{j}(i \omega)}=\frac{H_{x_{o} f_{k}}(i \omega) F_{k}(i \omega)}{H_{x_{j} f_{k}}(i \omega) F_{k}(i \omega)}=\frac{N_{o k}(i \omega) / D_{o k}(i \omega)}{N_{j k}(i \omega) / D_{j k}(i \omega)}=\frac{N_{o k}(i \omega) D_{j k}(i \omega)}{N_{j k}(i \omega) D_{o k}(i \omega)}
$$

Em que, $N_{o k}(i \omega), N_{j k}(i \omega)$ são os polinômios do numerador e $D_{j k}(i \omega), D_{o k}(i \omega)$ são os polinômios do denominador nos modelos das funções de transferência $H_{o k}(i \omega)=\frac{N_{o k}(i \omega)}{D_{o k}(i \omega)}$ e $H_{j k}(i \omega)=\frac{N_{j k}(i \omega)}{D_{j k}(i \omega)}$. Pode-se notar que as raízes dos polinômios $D_{j k}(i \omega)$ e $D_{o k}(i \omega)$ da função de transferência contêm os pólos ou frequências naturais do sistema, os quais podem desaparecer por estabelecer uma relação de duas respostas. No entanto, os polinômios $D_{j k}(i \omega)$ e $D_{o k}(i \omega)$ podem conter diferentes quantidades de pólos e não todos desaparecem, pelo contrário estabelecem-se como zeros ou pólos da função de transmissibilidade, respectivamente.

Em resumo, os pólos da função de transmissibilidade serão iguais aos zeros da função de transferência $H_{j k}(i \omega)$ ou as raízes do numerador $N_{j k}(i \omega)$ e aos pólos que não desapareçam do denominador $D_{o k}(i \omega)$ ao fazer a relação com ${ }_{D_{j k}(i \omega)}$. Então, geralmente não todos os picos da função de transmissibilidade coincidem com as ressonâncias ou pólos do sistema.

Assim, fazendo uso do modelo da função de transferência para sistemas LTI-MDF com amortecimento viscoso geral (Equação (2-52)) pode-se representar a função de transmissibilidade da Equação (2-54), como.

$$
T^{k}{ }_{x_{o} x_{j}}(i \omega)=\frac{H_{x_{o j k}}(i \omega)}{H_{x_{j j k}}(i \omega)}=\frac{\sum_{l=1}^{N}\left(\frac{\Psi_{o l} \Psi_{k l}}{a_{l}\left(i \omega-\lambda_{l}\right)}+\frac{\Psi_{o l}{ }^{*} \Psi_{k l}{ }^{*}}{a_{l}^{*}\left(i \omega-\lambda_{l}{ }^{*}\right)}\right)}{\sum_{l=1}^{N}\left(\frac{\Psi_{j l} \Psi_{k l}}{a_{l}\left(i \omega-\lambda_{l}\right)}+\frac{\Psi_{j l}{ }^{*} \Psi_{k l}{ }^{*}}{a_{l}{ }^{*}\left(i \omega-\lambda_{l}{ }^{*}\right)}\right)}
$$

Uma importante propriedade da função de transmissibilidade é quando a variável i $\omega$ tende a um pólo do sistema $\lambda_{r}$ (Devriendt e Guillaume, 2008). Nesse limite é possível notar a primeira vista que converge em um valor de infinito sobre infinito, mas se é usado a regra de L’Hospital para o cálculo do limite, obtém-se a seguinte convergência . 


$$
\begin{aligned}
& \lim _{i \omega \rightarrow \lambda_{r}} T_{{ }^{\prime} \alpha_{j} \alpha_{j}}^{k}(i \omega)=\frac{\lim _{i \omega \rightarrow \lambda_{r}} \sum_{l=1}^{N}\left(\frac{\Psi_{o l} \Psi_{k l}}{a_{l}\left(i \omega-\lambda_{l}\right)}+\frac{\Psi_{o l}{ }^{*} \Psi_{k l}{ }^{*}}{a_{l}^{*}\left(i \omega-\lambda_{l}{ }^{*}\right)}\right)}{\lim _{i \omega \rightarrow \lambda_{r}} \sum_{l=1}^{N}\left(\frac{\Psi_{j l} \Psi_{k l}}{a_{l}\left(i \omega-\lambda_{l}\right)}+\frac{\Psi_{j l}{ }^{*} \Psi_{k l}{ }^{*}}{a_{l}^{*}\left(i \omega-\lambda_{l}{ }^{*}\right)}\right)}=\frac{\infty}{\infty} \\
& =\frac{\lim _{i \omega \rightarrow \lambda_{r}}\left(\frac{\partial}{\partial \lambda_{r}}\left(\sum_{l=1}^{N}\left(\frac{\Psi_{o l} \Psi_{k l}}{a_{l}\left(i \omega-\lambda_{l}\right)}+\frac{\Psi_{o l}{ }^{*} \Psi_{k l}{ }^{*}}{a_{l}{ }^{*}\left(i \omega-\lambda_{l}{ }^{*}\right)}\right)\right)\right)}{\lim _{i \omega \rightarrow \lambda_{r}}\left(\frac{\partial}{\partial \lambda_{r}}\left(\sum_{l=1}^{N}\left(\frac{\Psi_{j l} \Psi_{k l}}{a_{l}\left(i \omega-\lambda_{l}\right)}+\frac{\Psi_{j l}{ }^{*} \Psi_{k l}{ }^{*}}{a_{l}{ }^{*}\left(i \omega-\lambda_{l}{ }^{*}\right)}\right)\right)\right)}=\frac{\lim _{i \omega \rightarrow \lambda_{r}}\left(\frac{\Psi_{o l} \Psi_{k l}}{a_{l}\left(i \omega-\lambda_{r}\right)^{2}}\right)}{\lim _{i \omega \rightarrow \lambda_{r}}\left(\frac{\Psi_{j l} \Psi_{k l}}{a_{l}\left(i \omega-\lambda_{r}\right)^{2}}\right)} \\
& =\frac{\Psi_{o r}}{\Psi_{j r}}
\end{aligned}
$$

Pode-se observar que o limite na Equação (2-56) é independente da localização da entrada $k$ e da força de excitação. Esse aspecto é de grande utilidade para a construção da configuração de um modo de vibração, $r$, a partir do conhecimento de diferentes funções de transmissibilidade do sistema associadas a um ponto de referência. Por exemplo, considerese uma estrutura na qual foi feita uma medição simultânea em 5 pontos diferentes, com esses dados de resposta se podem construir 5 funções de transmissibilidade com referência ao ponto 1. Assim, aplicando a propriedade mostrada na Equação (2-56), pode-se encontrar a configuração do modo de vibração para uma frequência, $r$, como.

$$
\lim _{i \omega \rightarrow \lambda_{r}}\left[T^{k}{ }_{11}(i \omega), T^{k}{ }_{21}(i \omega), T^{k}{ }_{31}(i \omega), T^{k}{ }_{41}(i \omega), T^{k}{ }_{51}(i \omega)\right]^{T}=\frac{1}{\Psi_{1 r}}\left[\Psi_{1 r}, \Psi_{2 r}, \Psi_{3 r}, \Psi_{4 r}, \Psi_{5 r}\right]^{T}
$$

Para o caso de vibrações aleatórias a construção da função de transmissibilidade é determinada fazendo uso da Equação (2-8) no domínio da frequência, $S_{x_{k} x_{j}}(i \omega)=H_{x_{k} f_{k}}(i \omega) S_{f_{k} x_{j}}(i \omega)$, e estabelecendo a saída $x_{j}$ na mesma coordenada da força $f_{k}$, e igualando à Equação (2-16). Dessa forma, obtém-se a seguinte relação.

$$
\begin{aligned}
T^{k}{ }_{x_{o} x_{j}}(i \omega)= & \frac{H_{x_{o} f_{k}}(i \omega)}{H_{x_{j} f_{k}}(i \omega)}=\frac{S_{x_{0} x_{k}}(i \omega) / S_{f_{k} x_{k}}(i \omega)}{S_{x_{j} x_{k}}(i \omega) / S_{f_{k} x_{k}}(i \omega)}=\frac{S_{x_{x_{0}} x_{k}}(i \omega)}{S_{x_{j} x_{k}}(i \omega)} \\
= & \frac{\sum_{r=1}^{N} \sum_{s=1}^{N} H^{*}{ }^{*}{ }_{x_{o} f_{r}}(i \omega) \cdot H_{x_{k} f_{s}}(i \omega) \cdot S_{f_{r} f_{s}}(i \omega)}{\sum_{r=1}^{N} \sum_{s=1}^{N} H^{*}{ }^{*}{ }_{x_{j} f_{r}}(i \omega) \cdot H_{x_{k} f_{s}}(i \omega) \cdot S_{f_{r} f_{s}}(i \omega)}
\end{aligned}
$$

O limite quando $i \omega$ tende $a \lambda_{r}$ na função de transmissibilidade para vibrações aleatórias (Equação (2-58)) converge para o mesmo resultado da Equação (2-56). Portanto, o uso de funções de transmissibilidade com densidades espectrais pode ser também 
utilizado para a construção de modos de vibração, da mesma forma como foi mostrado na Equação (2-57).

Uma extensão do conceito de transmissibilidade para sistemas de múltiplos graus de liberdade se pode estabelecer, a partir de definir as seguintes variáveis: um vetor $F_{A}$ que contém as magnitudes das forças aplicadas ao sistema nas coordenadas $A$, um vetor $X_{K}$ com amplitudes conhecidas da resposta nas coordenadas $K$, e um vetor $X_{U}$ com amplitudes desconhecidas da resposta nas coordenadas $U$ (Ribeiro, Silva e Maia, 2000). Com essas variáveis definem-se as seguintes relações.

$$
\begin{aligned}
& \left\{X_{u}(i \omega)\right\}=\left\lfloor H_{x_{u} f_{a}}(i \omega)\right\rfloor\left\{F_{a}(i \omega)\right\} \\
& \left\{X_{k}(i \omega)\right\}=\left\lfloor H_{x_{k} f_{a}}(i \omega)\right\rfloor\left\{F_{a}(i \omega)\right\}
\end{aligned}
$$

Em que $H_{x_{u} f_{a}}(i \omega)$ e $H_{x_{k} f_{a}}(i \omega)$ são as funções de transferência relacionando coordenadas $U-A$, e $K-A$, respectivamente. Substituindo a Equação (2-60), em (2-59) se segue que.

$$
\left\{X_{u}(i \omega)\right\}=\left[H_{x_{u} f_{a}}(i \omega)\right]\left[H_{x_{k} f_{a}}(i \omega)\right]^{+}\left\{X_{k}(i \omega)\right\}
$$

ou

$$
\left\{X_{u}(i \omega)\right\}=\left[T_{x_{u} x_{k}}^{a}(i \omega)\right]\left\{X_{k}(i \omega)\right\}
$$

Em que $\left[H_{x_{k} f_{a}}(i \omega)\right]^{+}$é a pseudo-inversa de $H_{x_{k} f_{a}}(i \omega)$. Assim, a matriz de transmissibilidade é definida, como.

$$
\left[T^{a}{ }_{x_{u} x_{k}}(i \omega)\right]=\left[H_{x_{u} f_{a}}(i \omega)\right]\left[H_{x_{k} f_{a}}(i \omega)\right]^{+}
$$

Uma única restrição na Equação (2-63), para que a pseudo-inversa à esquerda exista, é que o número de coordenadas de $K$ deve ser maior ou igual que o número de coordenadas de $A$.

No caso de vibrações aleatórias podem ser considerados igualmente dois subconjuntos de respostas desconhecidas $U$ e as respostas conhecidas $K$ para um conjunto de forças $A$ (Fontul et al., 2004). Assim, tendo-se em conta a Equação (2-8) no domínio da frequência da forma matricial podem-se escrever as seguintes relações.

$$
\begin{aligned}
& \left.\left[S_{x_{k} x_{k}}(i \omega)\right]=\left[S_{x_{k} f_{a}}(i \omega)\right] H_{x_{k} f_{a}}(i \omega)\right]^{T} \\
& \left.\left[S_{x_{k} x_{u}}(i \omega)\right]=\left[S_{x_{k} f_{a}}(i \omega)\right] H_{x_{u} f_{a}}(i \omega)\right]^{T}
\end{aligned}
$$


Da Equação (2-64) verifica-se que.

$\left[S_{x_{k} f_{a}}(i \omega)\right]^{T}=\left[H_{x_{k} f_{a}}(i \omega)\right]^{+}\left[S_{x_{k} x_{k}}(i \omega)\right]^{T}$

Combinando a Equação (2-66) e (2-65), leva a.

$\left[S_{x_{k} x_{u}}(i \omega)\right]^{T}=\left[H_{x_{u} f_{a}}(i \omega)\right]\left[H_{x_{k} f_{a}}(i \omega)\right]^{+}\left[S_{x_{k} x_{k}}(i \omega)\right]^{T}$

A expressão (2-67) relaciona a matriz de densidade espectral cruzada entre a resposta desconhecida e a conhecida com a matriz de densidade espectral entre respostas conhecidas. Nota-se que a mesma matriz de transmissibilidade obtida em vibrações harmônicas (Equação (2-63)) é obtida para o caso de vibrações aleatórias.

$\left[S_{x_{k} x_{u}}(i \omega)\right]^{T}=\left[T^{a}{ }_{x_{u} x_{k}}(i \omega)\right]\left[S_{x_{k} x_{k}}(i \omega)\right]^{T}$

\subsection{CONSIDERAÇÕES FINAIS.}

Notem-se duas suposições importantes em OMA: 1) uma excitação de tipo ruído branco que garante que as frequências naturais do sistema serão excitadas, além de que outro tipo de frequências provenientes da excitação não será incluído na saída, e, 2) a excitação deve estar adequadamente distribuída na estrutura com o fim de excitar todos os modos de vibração. A partir destas duas suposições é possível avaliar diretamente os parâmetros modais importantes do sistema com somente a informação da saída.

Dessa forma, ressaltam-se duas variáveis necessárias para estimar parâmetros modais com a informação de saída e referem-se principalmente a resposta espectral e as funções de correlação da resposta. Assim, pode-se estabelecer de modo geral que a base principal dos métodos de identificação modal para OMA no domínio da frequência encontrase na definição da densidade espectral da resposta $\left[s_{y}(\omega)\right]$, e para os métodos no domínio do tempo na definição das funções de correlação da resposta $r_{y}(k)$.

Além disso, salienta-se a importância da propriedade da transmissibilidade quando tende a um pólo do sistema, a qual converge a uma relação de amplitudes de modos vibração que não depende do espectro da excitação. Essa propriedade será retomada posteriormente no capítulo da revisão de métodos de identificação modal no domínio da frequência, no qual se trataram metodologias que usam essa propriedade com o intuito de avaliar parâmetros modais usando somente a resposta do sistema, com a possibilidade de se ter uma independência das características das forças de excitação. 



\section{CAPÍTULO 3. REVISÃO DE MÉTOdOS NO DOMINIO DA FREQUÊNCIA PARA A ANÁLISE MODAL OPERACIONAL.}

Nos anos 90 foi proposto o uso da excitação natural para a identificação de parâmetros modais (Natural Excitation Technique-NExT) (James, Carne e Lauffer, 1993). O método propõe que a função de correlação entre dois sinais de um sistema linear com modos normais sujeita a uma entrada de ruído branco é da mesma forma que uma resposta impulsiva, aspecto exposto e demostrado na Equação (2-22). Dessa forma, em NExT se define que a função de correlação pode ser expressa como uma somatória de senos em decaimento (sinal transiente), na qual cada sinal sinusoidal tem uma frequência amortecida, um fator de amortecimento e um coeficiente da forma modal que é idêntica ao correspondente modo estrutural. Portanto, um dos principais parâmetros em definir na identificação modal em OMA são as funções de correlação. Assim, três diferentes métodos para a obtenção das funções de correlação serão abordados. Com essas funções de correlação podem ser obtidas diretamente as funções de densidade espectral a partir de aplicar a transformada de Fourier. No entanto, melhores estimativas das funções de densidade espectral podem ser obtidas a partir de aplicar uma média espectral de diferentes sub-amostras sobrepostas do sinal. A técnica clássica usada nesse sentido é o método de Welch, descrito neste capítulo.

A partir da base da definição das funções de densidade espectral, serão descritas diferentes metodologias no domínio da frequência para extrair parâmetros modais do sinal. Ditas metodologias serão detalhadas analisando suas principais suposições, as técnicas matemáticas e o procedimento utilizado, assim como suas limitações e vantagens. Dentro dessas metodologias serão estudadas duas metodologias recentes que não consideram a suposição de ruído branco como entrada ao sistema, as quais são baseadas em conceitos de transmissibilidade.

Finalmente, algumas considerações finais serão dadas, tentando abordar de maneira geral os tópicos não considerados em detalhe na revisão. 


\subsection{FUNÇÕES DE CORRELAÇÂO DE DADOS DE RESPOSTA}

\subsubsection{Método direto.}

No Capítulo 2 foi introduzido o conceito de correlação entre sinais, no qual foi apresentada uma formulação para estimar a função de correlação para dados discretos finitos, como.

$\hat{R}_{x_{k} x_{j}}(k)=\frac{1}{N} \sum_{n=0}^{N-1-k} x_{k}(n) x_{j}(n+k) \quad k=0,1, \ldots ., N-1$

Essa correlação se pode representar em termos de densidade espectral ou seu periodograma no domínio da frequência, a partir de aplicar a transformada de Fourier, estabelecendo assim uma relação, como (Strum e Kirk, 1999).

$$
F\left[\hat{R}_{x_{k} x_{j}}(k)\right]=\hat{S}_{x_{k} x_{j}}(i \omega)=\frac{1}{N} X_{k}(-i \omega) \cdot X_{j}(i \omega)=\frac{1}{N} X_{k}(i \omega)^{*} \cdot X_{j}(i \omega)
$$

Então, a partir de usar a transformada inversa de Fourier na Equação (3-2) pode-se avaliar da mesma forma a função de correlação entre dois sinais.

$$
\hat{R}_{x_{k} x_{j}}(k)=F^{-1}\left[\frac{1}{N} X_{k}(i \omega)^{*} \cdot X_{j}(i \omega)\right]
$$

Assim, se é usado o algoritmo rápido para o cálculo da transformada de Fourier (Fast Fourier Transform-FFT) (Cooley e Tukey, 1965) o custo computacional de se avaliar a Equação (3-3) será menor que o cálculo da somatória da Equação (3-1). No entanto, o estimador da função de correlação baseado no método direto ou cálculo direto do periodograma é um estimador enviesado da função de correlação, já que o valor esperado do estimador não é igual ao valor verdadeiro da função de correlação (Hayes, 1996).

$$
E\left[\hat{R}_{x_{k} x_{j}}(k)\right]=\frac{N-|k|}{N} R_{x_{k} x_{j}}(k)=\varpi(k) R_{x_{k} x_{j}}(k)
$$

Em que, $\varpi(k)$ é conhecida como a janela triangular de Barlett. O estimador também é considerado assintoticamente não enviesado para $\mathrm{N}$ quando tende ao infinito, em que a relação $\frac{N-|k|}{N}$ tende a 1. Portanto, se $\mathrm{N}$ é finito um procedimento para eliminar o viés da função correlação é dividir a estimação da função de correlação por uma janela de Barlett do tamanho da função de correlação. 
Além disso, o método direto é um estimador não consistente das funções de densidade espectral e não deve ser usado para a análise espectral, devido a que a variância do estimador não decresce a zero quando $\mathrm{N}$ tende ao infinito (Hayes, 1996).

$\operatorname{Var}\left[\hat{S}_{x_{k} x_{j}}(i \omega)\right]=S^{2}{ }_{x_{k} x_{j}}(i \omega)$

\subsubsection{Método baseado no periodograma médio.}

Uma alternativa do cálculo da função de correlação é usar uma média da densidade espectral da resposta, a partir de definir $\boldsymbol{A}$ amostras não sobrepostas de comprimento $\boldsymbol{L}$ nos sinais $x_{k}$ e $x_{j}$, assim o número de dados total do sinal fica igual a $\boldsymbol{N}=\boldsymbol{A} \boldsymbol{L}$. Desse modo, podem-se estabelecer $\boldsymbol{q}$ diferentes funções de correlação dos mesmos sinais $x_{k}$ e $x_{j}$ variando entre 1 e A amostras, como.

$\hat{R}_{x_{k} x_{j}}(k)_{q}=\frac{1}{L} \sum_{n=0}^{L-1-k} x_{k}(n+q L) x_{j}(n+q L+k) \quad k=0,1, \ldots, L-1$

E diferentes densidades espectrais $\boldsymbol{q}$ aplicando a transformada de Fourier

$$
F\left[\hat{R}_{x_{k} X_{j}}(k)_{q}\right]=\hat{S}_{x_{k} X_{j}}(i \omega)_{q}=\frac{1}{L}\left(X_{k}(i \omega)^{*} \cdot X_{j}(i \omega)\right)_{q}
$$

Avaliando uma média aritmética das densidades espectrais $\boldsymbol{q}$ obtém-se um periodograma médio, assim.

$$
\hat{S}_{x_{k} X_{j}}(i \omega)=\frac{1}{A} \sum_{q=1}^{A} \frac{1}{L}\left(X_{k}(i \omega)^{*} \cdot X_{j}(i \omega)\right)_{q}
$$

Uma estimativa da função de correlação pode ser obtida a partir de se avaliar a transformada inversa de Fourier da Equação (3-8), como.

$$
\hat{R}_{x_{k} x_{j}}(k)=F^{-1}\left[\frac{1}{A} \sum_{q=1}^{A} \frac{1}{L}\left(X_{k}(i \omega)^{*} \cdot X_{j}(i \omega)\right)_{q}\right]
$$

O estimador da função de correlação por meio do método do periodograma médio é também um estimador enviesado e assintoticamente não enviesado do mesmo modo que o método direto, portanto, suas estimações das funções de correlação devem ser também divididas por uma janela de Barlett para eliminar o viés da função de correlação. Porém, o 
periodograma médio é um estimador consistente das funções de densidade espectral, já que a variância da estimação da densidade espectral tende ao zero como $\boldsymbol{A}$ tende ao infinito (Hayes, 1996).

$\operatorname{Var}\left[\hat{S}_{x_{k} x_{j}}(i \omega)\right] \approx \frac{1}{A} S^{2}{ }_{x_{k} x_{j}}(i \omega)$

De fato, as funções de correlação podem ser estimadas a partir de qualquer estimação das funções de densidade espectrais usando a relação de Wiener-Khintchine, avaliadas através da transformada inversa de Fourier. Assim, as funções de correlação podem ser avaliadas usando outros tipos de estimadores das funções de densidade espectral como o periodograma modificado com aplicação de janelas na série de dados e o periodograma médio modificado ou método de Welch (1967) que além de aplicar janelas, faz uma sobreposição dos dados para o cálculo médio das densidades espectrais. O método de Welch será retomado posteriormente quando se aborde o método para avaliação da função de densidade espectral.

\subsubsection{Método do decremento aleatório.}

O método do decremento aleatório (Random Decrement- RD) é uma técnica de processamento aproximado do sinal no domínio do tempo para a construção de um sinal característico, a partir da média de um conjunto de segmentos pré-selecionados do sinal. $\mathrm{O}$ sinal característico é comumente referido como de sinal RD. O sinal RD representa a vibração livre do sistema dinâmico sob suposição de uma excitação de ruído branco e determinadas condições iniciais (Asmussen, J. C., Ibrahim, S. e Brincker, R., 1998). Os princípios detrás do método do decremento aleatório são descritos, como segue.

No Capítulo 2 foi exposta a resposta de um sistema dinâmico LTI como uma soma de convoluções para condições iniciais de deslocamento e velocidade iguais a zero (Equação 2-2), mas uma resposta do sistema com condições iniciais pode ser representada, como (He et al., 2011).

$x_{k}(n)=\sum_{s=1}^{N} x_{s}(0) \cdot D_{x_{k} x_{s}(0)}(n)+\sum_{s=1}^{N} \dot{x}_{s}(0) \cdot V_{x_{k} \dot{x}_{s}(0)}(n)+\sum_{s=1}^{N} h_{x_{k} f_{s}}(n) \times f_{s}(n)$

Em que $D_{x_{k} x_{s}(0)}(n)$ é a resposta livre do sistema em $\boldsymbol{k}$ por uma condição inicial de deslocamento em $\boldsymbol{s}, V_{x_{k} \dot{x}_{s}(0)}(n)$ é a resposta livre do sistema em $\boldsymbol{k}$ por uma condição inicial 
de velocidade em $\boldsymbol{s}, h_{x_{k} f_{s}}$ é a resposta impulsiva em $\boldsymbol{k}$ por uma força de impulso em $\boldsymbol{s}, x_{s}(0)$ e $\dot{x}_{s}(0)$ são condições iniciais de deslocamento e velocidade, respectivamente.

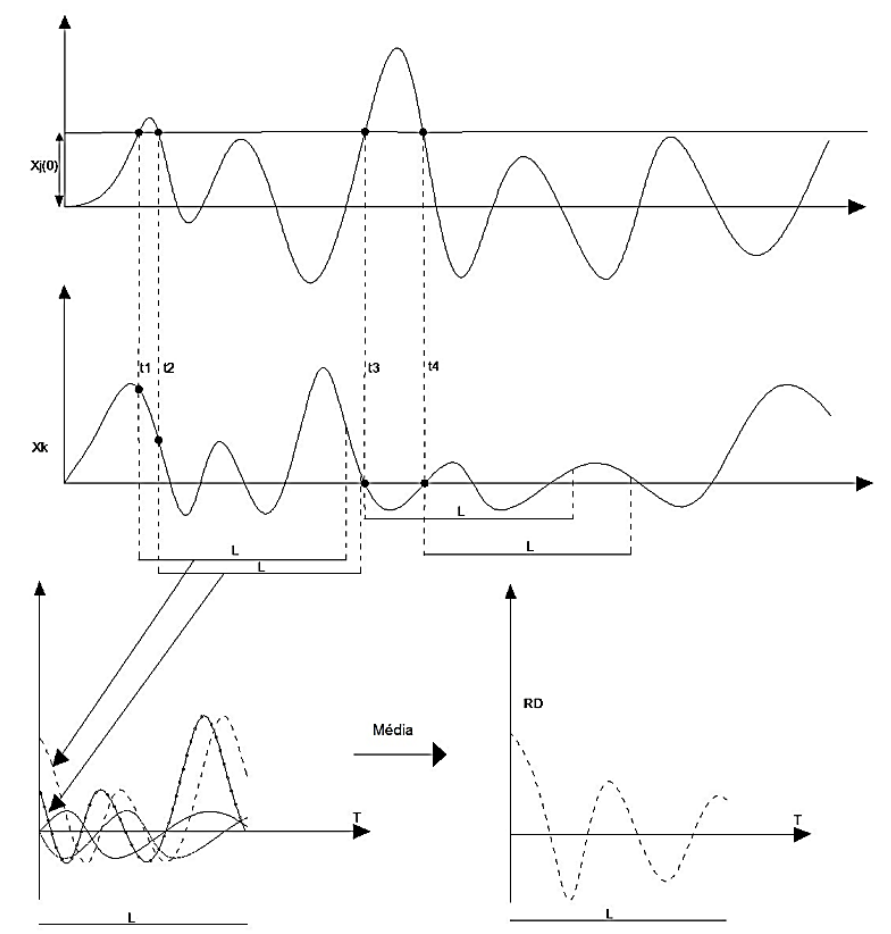

Figura 3-1 Seleção de segmentos, método do decremento aleatório.

Como se pode notar na Equação (3-11) a resposta dinâmica de um sistema $x(n)$ em qualquer tempo $\boldsymbol{n}$ é dada pela combinação de três contribuições: 1) a resposta degrau devido a uma condição inicial de deslocamento, 2) a resposta impulsiva causada pela condição inicial de velocidade e 3) a resposta aleatória devido a uma excitação aleatória $f(n)$ aplicada no sistema.

Então, se é suposto que $f(n)$ é um ruído branco de origem gaussiana com média zero, então uma média de diferentes segmentos de resposta fará que a parte aleatória da resposta seja zero, $E[f(n)]=0$. Para garantir que a parte de resposta livre não seja zero, uma condição para a seleção dos segmentos deve ser aplicada no sinal. Por exemplo, como é mostrado na Figura $3-1$, se é determinado um comprimento arbitrário $L$ do sinal e é selecionado um valor apropriado $x_{j}(0)$ no sinal $x_{j}$ interceptará o sinal $x_{k}$ em tempos denotados como $t_{i}(i=1,2,3 \ldots)$. Assim, são extraídos diferentes segmentos $L$ no sinal $x_{k}$ para realizar uma média e obter a resposta livre do sistema. 
Além disso, sob ação de $f(n)$, a resposta de $x(n)$ do sistema é também um processo gaussiano de média zero, portanto, para uma condição de $x_{j}(0)$ fixa, a velocidade média dos segmentos será igual a zero $E\left[\dot{x}_{s}(0)\right]=0$, igualmente as demais condições inicias de deslocamento $x_{s}(0)$ diferente a $x_{j}(0)$ serão iguais a zero, $E\left[x_{s}(0)\right]=x_{j}(0)$. Desse modo, o valor esperado de $x(n)$ obtido fica igual a.

$$
\begin{aligned}
& E\left[X_{k}(n)\right]=\sum_{s=1}^{N} E\left[X_{s}(0)\right] \cdot D_{x_{k} x_{s}(0)}(n)+\sum_{s=1}^{N} E\left[\dot{x}_{s}(0)\right] \cdot V_{x_{k} \dot{x}_{s}(0)}(n)+\sum_{s=1}^{N} h_{x_{k} f_{s}}(n) \times E\left[f_{s}(n)\right] \\
& E\left[X_{k}(n)\right]=X_{j}(0) D_{x_{k} x_{j}(0)}(n)
\end{aligned}
$$

Na Equação (3-12) mostra-se que o valor esperado do sinal $x_{k}(n)$ é a resposta livre do sistema com deslocamento inicial $x_{j}(0)$ e velocidade inicial zero. Então, no contexto prático o número de amostras é limitado, portanto, é usada a média aritmética de diferentes segmentos como aproximação do valor esperado do sinal (Ibrahim, 2001). Assim, as funções RD podem ser computadas, como.

$$
R D_{x_{k} x_{j}}(t)=\frac{1}{N} \sum_{i=1}^{N} x_{k}\left(t_{i}+L\right) \mid T_{x_{j}}\left(t_{i}\right)
$$

Em que $\mathrm{N}$ é o número de segmentos extraídos da resposta dinâmica ${ }_{x_{k}}$ e $T_{x_{j}}\left(t_{i}\right)$ é a condição em $x_{j}$ para extrair os segmentos. Além da condição de uma passagem de nível determinado em $x_{j}$, outro tipo de condições podem ser estabelecidas, tais como: passagem de pontos positivos, passagem de zeros e extremos locais (Asmussen, 1997). Assim, as diferentes condições, podem ser.

$T_{x_{j}}\left(t_{i}\right)=\left\{a_{1} \leq x_{j}(t) \leq a_{2}, v_{1} \leq \dot{x}_{j}(t) \leq v_{2}\right\} \quad$ condição geral

$T_{x_{j}}\left(t_{i}\right)=\left\{x_{j}(t)=a_{1},-\infty \leq \dot{x}_{j}(t) \leq \infty\right\} \quad$ passagem de nível

$T_{x_{j}}\left(t_{i}\right)=\left\{a_{1} \leq x_{j}(t) \leq a_{2},-\infty \leq \dot{x}_{j}(t) \leq \infty\right\}$ passagem de pontos positivos

$T_{x_{j}}\left(t_{i}\right)=\left\{x_{j}(t)=0,0 \leq \dot{x}_{j}(t) \leq \infty\right\} \quad$ passagem de zeros

$T_{x_{j}}\left(t_{i}\right)=\left\{0 \leq x_{j}(t) \leq \infty, \dot{x}_{j}(t)=0\right\} \quad$ extremos locais

Como nas aplicações práticas existem problemas tais como: o ruído no sinal e limitações no tempo de aquisição, umas das condições para calcular a média que produz os melhores resultados na avaliação das funções RD é a condição de passagem de pontos positivos, principalmente devido a que a quantidade de segmentos para o cálculo da média 
é maior que nas outras condições (Asmussen, J., Ibrahim, S. e Brincker, R., 1998). Um exemplo desse fato é o trabalho de Ye, Feng et al. (2012), no qual foi mostrado que para uma resposta total de 10 seg com uso da condição de pontos positivos limitados entre 1/3 e 1/2 da máxima amplitude resulta uma diminuição notável do ruído para o cálculo da função $\mathrm{RD}$ de um comprimento de 2 seg, comparado com o cálculo para uma condição de passagem de nível.

Se as respostas $x_{k}$ e $x_{j}$ são processos estacionários gaussianos de média zero e é aplicada a condição geral (Equação (3-14)), existe uma relação fundamental entre as funções RD e as funções de correlação e suas derivadas, dada como (Brincker et al., 1992; Asmussen, J. C., Ibrahim, S. e Brincker, R., 1998).

$$
R D_{x_{k} x_{j}}(t)=\frac{R_{x_{k} x_{j}}(t)}{\sigma_{x_{k}}{ }^{2}} a-\frac{\dot{R}_{x_{k} x_{j}}(t)}{{\sigma_{\dot{x}_{k}}}^{2}} v
$$

Em que, $\sigma_{x_{k}}{ }^{2}$ é a covariância do processo $x_{k}$ e os níveis a e $\boldsymbol{v}$ são determinados da função de densidade de probabilidade $x_{k}, f_{x_{k}}(x)$, como.

$$
a=\frac{\int_{a_{1}}^{a_{2}} x_{k} f_{x_{k}}(x) d x}{\int_{a_{1}}^{a_{2}} f_{x_{k}}(x) d x} \quad v=\frac{\int_{v_{1}}^{v_{2}} \dot{x}_{k} f_{\dot{x}_{k}}(x) d \dot{x}}{\int_{v_{1}}^{v_{2}} f_{\dot{x}_{k}}(x) d \dot{x}}
$$

Finalmente, podem-se identificar três parâmetros importantes para a determinação das funções RD: o tipo de condição usada para a seleção dos segmentos $T_{x_{j}}\left(t_{i}\right)$, os níveis selecionados da respectiva condição $a_{1}, a_{2}, v_{1}, v_{2}$ e a seleção do tamanho do segmento $L$.

\subsection{FUNÇÕES DE DENSIDADE ESPECTRAL.}

A determinação das funções de densidade espectral é a base para as metodologias no domínio da frequência, portanto, nesta seção são apresentadas as metodologias tradicionalmente usadas para estimação de ditas funções a partir de série temporais e o uso da transformada de Fourier. 
Entre os diferentes métodos não paramétricos para determinar as funções de densidade espectral, o método mais usado é o método de Welch, (1967) que envolve a determinação da transformada de Fourier dos sinais de resposta.

O método de Welch estabelece duas modificações ao método de periodograma médio ou método de Barlett: 1) permite que as amostras de realizações randômicas $x_{k}(n)$ e $x_{j}(n)$ sejam sobrepostas e 2) permite aplicar janelas $\varpi(n)$ a cada amostra de dados.

No caso, quando é aplicada uma janela $\varpi(n)$ ao processo randômico é chamado como de periodograma modificado e se define, como.

$$
\hat{R}_{x_{k} x_{j}}(k)=\frac{1}{N} \sum_{n=0}^{N-1-k} \varpi(n) x_{k}(n) \varpi(n) x_{j}(n+k) \quad k=0,1, \ldots ., N-1
$$

Aplicando a transformada de Fourier na sequência de correlação a seguinte densidade espectral é obtida.

$$
F\left[\hat{R}_{x_{k} x_{j}}(k)\right]=\hat{S}_{x_{k} x_{j}}(i \omega)=\frac{1}{N U} X u_{k}(i \omega)^{*} \cdot X u_{j}(i \omega) \text { com } U=\frac{1}{N} \sum_{n=0}^{N-1}|\varpi(n)|^{2}
$$

Em que, $X u_{k}(i \omega)$ e $X u_{j}(i \omega)$ são a transformada de Fourier de $\varpi(n) x_{k}(n)$ e $\varpi(n) x_{j}(n)$, respectivamente. Com o intuito de que não exista uma alteração da variância do processo randômico quando são aplicadas janelas temporais, as ordenadas da transformada de Fourier devem ser dividas pelo valor quadrático médio da janela $\boldsymbol{U}$.

Então, supondo que as amostras $x_{k}(n)$ e $x_{j}(n)$ estiverem deslocadas por $\boldsymbol{D}$ pontos e cada amostra tiver um comprimento $L, \boldsymbol{q}$ amostras vêm dadas por.

$$
\hat{R}_{x_{k} X_{j}}(k)_{q}=\frac{1}{L} \sum_{n=0}^{L-1-k} \varpi(n) x_{k}(n+q D) \varpi(n) x_{j}(n+q D+k) \quad k=0,1, \ldots ., L-1
$$

Assim, a quantidade de pontos sobrepostos entre $q$ e $q+1$ é $L-D$. Se houverem $\boldsymbol{A}$ amostras ao longo da totalidade dos pontos $\boldsymbol{N}$, então $\boldsymbol{N}$ fica como.

$$
N=L+D(A-1)
$$

Por exemplo, se $\boldsymbol{L}=\boldsymbol{D}$, então não existe uma sobreposição de pontos e $\boldsymbol{A}=\boldsymbol{N} / \boldsymbol{L}$, ficando igual ao método de periodograma médio. Igualmente, se as amostras estão sobrepostas um $50 \%$, fica $\boldsymbol{D}=\boldsymbol{L} / \mathbf{2}$ e $\boldsymbol{A}$ amostras, assim.

$$
A=2 \frac{N}{L}-1
$$


Finalmente, avaliando uma média aritmética das densidades espectrais $\boldsymbol{q}$ sobrepostas e com a aplicação de janelas, o periodograma pelo método de Welch se estabelece, como.

$$
\hat{S}_{x_{k} X_{j}}(i \omega)=\frac{1}{A} \sum_{q=1}^{A} \frac{1}{U L}\left(X u_{k}(i \omega)^{*} \cdot X u_{j}(i \omega)\right)_{q}
$$

Uma estimação das funções de correlação pode ser avaliada a partir de aplicação da transformada inversa de Fourier na Equação (3-22). O método de Welch é um estimador consistente das funções de densidade espectral devido a que sua variância tende a zero quando $\boldsymbol{N}$ tende ao infinito. Por exemplo, no caso de uma sobreposição dos dados do $50 \%$ e a aplicação de uma janela de Barlett, a variância do estimador se avalia, como (Hayes, 1996).

$\operatorname{Var}\left[\hat{S}_{x_{k} x_{j}}(i \omega)\right] \approx \frac{9 L}{16 N} S^{2}{ }_{x_{k} x_{j}}(i \omega)$

No contexto de aplicação de janelas no sinal, o tipo janela comumente usada para sinais aleatórios é a janela tipo Hanning. A formulação da janela de Hanning é dada, como (Oppenheim e Schafe, 1989).

$$
\begin{aligned}
& \varpi(n)=0.5\left(1-\frac{\cos (2 \pi n)}{N}\right), \quad 0 \leq n \leq N \\
& L=N+1
\end{aligned}
$$

Em que $L$ é o comprimento total da janela.

Nesse sentido, a aplicação de janelas no sinal aleatório tem uma grande utilidade para a redução do efeito de leakage ou distorção do espectro. O efeito de leakage é produto da existência de um número não inteiro de ciclos no sinal no interior do segmento; essa periodicidade é uma suposição importante do algoritmo da transformada de Fourier, a qual é usada em cada um dos segmentos no método de Welch, portanto, uma aplicação de janelas nos segmentos tem por objetivo garantir que o sinal a submeter à transformada de Fourier tenha um período inteiro, tendo um valor de zero no início e no final do segmento.

O método de Welch é concebido para reduzir a variância do periodograma a partir de aplicar uma média aritmética aos periodogramas. Outro método não paramétrico para reduzir a variabilidade do periodograma é o suavizado do periodograma ou método de Blackman e Tukey (1958). No método de Blackman e Tukey a variância do periodograma é 
reduzida a partir de aplicar uma janela à estimação da função de correlação $\hat{R}_{x_{k} x_{j}}(k)$ (Equação (3-3) e Equação (3-9)), com o objetivo de diminuir a contribuição de elementos não confiáveis da função de correlação. A razão se deve a que para valores grandes de $\boldsymbol{k}$ da função de correlação, as estimações usam menos pontos na estimação e, portanto, são menos confiáveis, pelo que deveriam ter menor peso na estimação do periodograma. Especificamente, a densidade espectral pelo método de Blackman e Tukey é dada por.

$$
\hat{S}_{x_{k} x_{j}}(i \omega)=\sum_{k=-\infty}^{\infty} \hat{R}_{x_{k} x_{j}}(k) \varpi(k) e^{-i \omega k}
$$

Em que, $\varpi(k)$ é a janela aplicada na estimação da função de correlação. Assim o método de Blackman e Tukey é um estimador consistente da função de densidade espectral decrescendo a variância quando $\mathrm{N}$ tende ao infinito (Hayes, 1996).

$\operatorname{Var}\left[\hat{S}_{x_{k} x_{j}}(i \omega)\right] \approx S^{2}{ }_{x_{k} x_{j}}(i \omega) \frac{1}{N} \sum_{k=-N}^{N}|\varpi(k)|^{2}$

Finalmente, foi mostrado que para uma excitação gaussiana com média zero as funções de decremento aleatório são proporcionais às funções de correlação (Equação (315)), portanto, podem ser usadas como estimadores das funções de correlação e das funções de densidade espectral a partir de aplicar a transformada de Fourier. Pode-se optar igualmente como na Equação (3-25) pela aplicação de uma janela temporal, como. $\hat{S}_{x_{k} x_{j}}(i \omega)=\sum_{k=-\infty}^{\infty} R D_{x_{k} x_{j}}(k) \varpi(k) e^{-i \omega k}$

\subsection{MÉTODOS NÃO PARAMÉTRICOS NO DOMÍNIO DA FREQUÊNCIA.}

A base principal dos métodos no domínio da frequência encontra-se na definição da densidade espectral das respostas do sistema, assim, diferentes alternativas com uso da transformada de Fourier foram expostas na secção anterior. Então, com base na hipótese que a excitação é do tipo ruído branco, no Capítulo 2 foi possível estabelecer que o cálculo da densidade espectral da resposta do sistema permite determinar diretamente as propriedades dinâmicas do sistema por sua proporcionalidade com a função de transferência (ver Equação (2-19)). Assim, as funções de densidade espectral apresentarão picos na amplitude que serão as frequências naturais de vibração amortecidas, as quais para um amortecimento baixo são muito próximas às frequências naturais não amortecidas (ver Equação (2-26)). 
Desta forma, serão apresentadas diversas metodologias de identificação modal no domínio da frequência analisando as limitações e as vantagens de metodologias tradicionais, bem como as mais recentes propostas baseadas em conceitos de transmissibilidade.

\subsubsection{Método de seleção de picos (PP).}

O método básico no domínio da frequência é a seleção de picos (Peak Picking -PP) que é aplicado há algumas décadas na identificação modal de estruturas de engenharia civil (Felber, 1993). Esse método é o mais adequado para fazer uma primeira revisão na qualidade dos dados e obter uma primeira ideia das propriedades dinâmicas do sistema. A estimação das frequências do sistema é baseada na construção da média normalizada da densidade espectral de potência (Average Normalized Power Spectral Densities- ANPSDs), na qual se podem identificar as frequências naturais do sistema através da escolha dos picos de ressonância no ANPSDs. Geralmente, a média dos espectros é feita a partir de somente os auto-espectros dos diferentes registros de sinais, através do procedimento seguinte.

$$
\operatorname{ANPSD}(i \omega)=\frac{1}{N} \sum_{k=1}^{N} N S_{x_{k} x_{k}}(i \omega)
$$

Em que, $\boldsymbol{N}$ é o número de graus de liberdade medidos e $N S_{x_{k} x_{k}}(i \omega)$ são os espectros normalizados. Os espectros normalizados são obtidos dividindo os auto-espectros pelas somas de suas respectivas amplitudes, como.

$$
N S_{x_{k} x_{k}}(i \omega)=\frac{S_{x_{k} x_{k}}(i \omega)}{\sum_{j=1}^{L} S_{x_{k} x_{k}}\left(i \omega_{j}\right)}
$$

A normalização dos dados tem como objetivo garantir uma igualdade no conteúdo energético dos diferentes auto-espectros calculados da série de resposta medidas, já que essas medições são feitas em pontos diferentes da estrutura com amplitudes diferentes de resposta e possivelmente também em instantes de tempos diferentes.

As formas modais nesse método são avaliadas a partir do uso das funções de transferência ou das funções de transmissibilidade entre vários pontos de medições da estrutura com respeito a um ponto de referência, da mesma forma como foi descrita no 
Capítulo 2 (Equação (2-57)). No entanto, as funções de transmissibilidade são calculadas a partir das densidades espectrais calculadas como na Equação (2-58), mas estabelecendo sempre um ponto de referência ref, como.

$T_{x_{j} x_{r e f}}^{r e f}(i \omega)=\frac{S_{x_{j} x_{r e f}}(i \omega)}{S_{x_{r e f} x_{r e f}}(i \omega)}$

Assim, exemplificando um sistema com 5 pontos podem-se construir 5 funções de transmissibilidade com referência a $S_{x_{\text {ref }} x_{\text {ref }}}(i \omega)$. Dessa forma, aplicando a propriedade mostrada na Equação (2-56) pode-se encontrar a configuração do modo de vibração para uma frequência, $r$, com funções de densidade espectral, como.

$$
\lim _{i \omega \rightarrow \lambda_{r}}\left[T^{k_{11}}(i \omega), T^{k}{ }_{21}(i \omega), T^{k}{ }_{31}(i \omega), T^{k}{ }_{41}(i \omega), T^{k}{ }^{k}(i \omega)\right]^{T}=\frac{1}{\Psi_{1 r}}\left[\Psi_{1 r}, \Psi_{2 r}, \Psi_{3 r}, \Psi_{4 r}, \Psi_{5 r}\right]^{T}
$$

Desse modo, a partir da escolha da frequência de ressonância nas diferentes funções de transmissibilidade são avaliados os valores de amplitude e de fase. A fase é utilizada para estabelecer o sentido da amplitude do modo, um valor de fase $0^{\circ}$ indica que $\mathbf{j}$ e ref estão no mesmo sentido, um valor de $180^{\circ}$ em sentido oposto.

A identificação das taxas de amortecimento no método PP é baseada na consideração que as frequências naturais ou picos nas funções de densidades espectrais são condicionados pela contribuição de um só modo ressonante, portanto, as vizinhanças da frequência de ressonância podem ser simuladas através de um oscilador de um só grau de liberdade com uma frequência $\omega_{l}$ e uma porcentagem de amortecimento $\xi_{l}$. A anterior aproximação só pode ser valida quando as frequências associadas aos diferentes modos de vibração encontram-se bem separadas e suas taxas de amortecimento são baixas.

O fato anterior pode ser demostrado pelo uso da Equação (2-37) que relaciona as saídas $\left\{X_{k}(i \omega)\right\}$ com as entradas $\left\{F_{r}(i \omega)\right\}$ a partir da Equação de contribuição modal (2-35), como.

$$
\left\{X_{k}(i \omega)\right\}=[\Phi]_{k l}|\hat{H}(i \omega)|_{z_{l} f_{r}}\left\{F_{r}(i \omega)\right\}
$$

Com 


$$
[\hat{H}(i \omega)]_{l_{l} f_{r}}=\frac{[\Phi]_{r l}}{\hat{M}_{l l}\left(\omega_{l}^{2}-\omega^{2}+2 i \xi_{l} \omega_{l} \omega\right)}
$$

Em que, $[\hat{H}(i \omega)]_{l_{l} f_{r}}$ é a contribuição do modo $Z_{l}$ para uma entrada em $\boldsymbol{r}$. Então, fazendo uma expansão da Equação (3-32) pode-se determinar que a resposta em termos de contribuição modal em um ponto $\boldsymbol{k}$ vem dada por.

$$
X_{k}(i \omega)=\phi_{k 1} \sum_{r=1}^{N} \hat{H}_{z_{1} f_{r}}(i \omega) F_{r}(i \omega)+\phi_{k 2} \sum_{r=1}^{N} \hat{H}_{z_{2} f_{r}}(i \omega) F_{r}(i \omega)+. .+\phi_{k N} \sum_{r=1}^{N} \hat{H}_{z_{N} f_{r}}(i \omega) F_{r}(i \omega)
$$

Sob suposição, que os modos são bem separados e as taxas de amortecimentos são baixas, a resposta em $\boldsymbol{k}$ na ressonância é dominada pela contribuição do correspondente modo de vibração $z_{l}$ e a contribuição dos outros modos podem ser despreciáveis, portanto, a resposta em $\boldsymbol{k}$ próximo à frequência de ressonância $i \omega_{l}$, pode-se avaliar aproximadamente, como.

$$
X_{k}\left(i \omega_{l}\right) \cong \phi_{k l} \sum_{r=1}^{N} \hat{H}_{z_{l} f_{r}}\left(i \omega_{l}\right) F_{r}\left(i \omega_{l}\right)
$$

Para a densidade espectral da resposta, caso de interesse, uma representação em termos de uma contribuição modal pode ser dada a partir de usar a Equação (3-33) e as equações de densidade espectral mostradas no Capítulo 2 (Equações (2-14) e (2-15)) estabelecendo as seguintes relações.

$$
\begin{aligned}
& S_{x_{k} x_{j}}(i \omega)=\phi^{*}{ }_{k 1} \sum_{r=1}^{N} \hat{H}_{z_{1} f_{r}}{ }^{*}(i \omega) S_{f_{r} x_{j}}(i \omega)+\phi^{*}{ }_{k 2} \sum_{r=1}^{N} \hat{H}_{z_{2} f_{r}}{ }^{*}(i \omega) S_{f_{r} x_{j}}(i \omega)+. .+\phi^{*}{ }_{k N} \sum_{r=1}^{N} \hat{H}_{z_{N} f_{r}}{ }^{*}(i \omega) S_{f_{r} x_{j}}(i \omega) \\
& S_{f_{r} x_{j}}(i \omega)=\phi_{j 1} \sum_{s=1}^{N} \hat{H}_{z_{1} f_{s}}(i \omega) S_{f_{r} f_{s}}(i \omega)+\phi_{j 2} \sum_{s=1}^{N} \hat{H}_{z_{2} f_{s}}(i \omega) S_{f_{r} f_{s}}(i \omega)+. .+\phi_{j N} \sum_{s=1}^{N} \hat{H}_{z_{N} f_{s}}(i \omega) S_{f_{r} f_{s}}(i \omega)
\end{aligned}
$$

Combinando as Equações (3-35) e (3-36), obtém-se uma representação da densidade espectral da resposta em termos de uma contribuição modal, assim. 


$$
\begin{aligned}
S_{x_{k} x_{j}}(i \omega)= & \phi_{k 1}^{*}\left[\sum_{r=1}^{N} \sum_{s=1}^{N} \hat{H}_{z_{1} f_{r}}{ }^{*}(i \omega) S_{f_{r} f_{s}}(i \omega) \hat{H}_{z_{1} f_{s}}(i \omega) \phi_{j 1}+. .+\sum_{r=1}^{N} \sum_{s=1}^{N} \hat{H}_{z_{1} f_{r}}{ }^{*}(i \omega) S_{f_{r} f_{s}}(i \omega) \hat{H}_{z_{N} f}(i \omega)_{s} \phi_{j N}\right]+ \\
& \phi^{*}{ }_{k 2}\left[\sum_{r=1}^{N} \sum_{s=1}^{N} \hat{H}_{z_{2} f_{r}}{ }^{*}(i \omega) S_{f_{r} f_{s}}(i \omega) \hat{H}_{z_{1} f_{s}}(i \omega) \phi_{j 1}+. .+\sum_{r=1}^{N} \sum_{s=1}^{N} \hat{H}_{z_{2} f_{r}}{ }^{*}(i \omega) S_{f_{r} f_{s}}(i \omega) \hat{H}_{z_{N} f_{s}}(i \omega) \phi_{j N}\right]+. . \\
& . .+\phi_{k N}^{*}\left[\sum_{r=1}^{N} \sum_{s=1}^{N} \hat{H}_{z_{N} f_{r}}{ }^{*}(i \omega) S_{f_{r} f_{s}}(i \omega) \hat{H}_{z_{1} f_{s}}(i \omega) \phi_{j 1}+. .+\sum_{r=1}^{N} \sum_{s=1}^{N} \hat{H}_{z_{N} f_{r}}{ }^{*}(i \omega) S_{f_{r} f_{s}}(i \omega) \hat{H}_{z_{N} f_{s}}(i \omega) \phi_{j N}\right]
\end{aligned}
$$

Uma representação compacta da Equação (3-37) é dada, como.

$$
S_{x_{k} x_{j}}(i \omega)=\sum_{l_{2}=1}^{N} \sum_{l_{1}=1}^{N} \sum_{r=1}^{N} \sum_{s=1}^{N} \Phi_{x_{k} z_{1}} \hat{H}_{z_{1} f_{r}}^{*}(i \omega) S_{f_{r} f_{s}}(i \omega) \hat{H}_{z_{12} f_{s}}(i \omega) \Phi_{x_{j} z_{12}}
$$

De igual forma, se os modos de vibração são bem separados e o amortecimento é baixo, a resposta $x_{k} x_{j}$ da densidade espectral próximo à frequência de ressonância $i \omega_{l}$ pode ser avaliada aproximadamente, como.

$$
S_{x_{k} x_{j}}\left(i \omega_{l}\right) \cong \phi^{*}{ }_{k l}^{N} \sum_{r=1}^{N} \sum_{s=1}^{N} \hat{H}_{z_{l} f_{r}}^{*}\left(i \omega_{l}\right) S_{f_{r} f_{s}}\left(i \omega_{l}\right) \hat{H}_{z_{l} f_{s}}\left(i \omega_{l}\right) \phi_{j l}
$$

Os termos $\hat{H}_{z_{l} f_{r}}{ }^{*}\left(i \omega_{l}\right)$ e $\hat{H}_{z_{l} f_{s}}\left(i \omega_{l}\right)$ respondem a um modelo de um sistema de um só grau de liberdade, portanto, é possível extrair o amortecimento com técnicas clássicas para sistemas de um só grau de liberdade, tais como: o método de largura de meia banda de potência (Chopra, 2001) e o método de ajuste do espectro teórico de um só grau de liberdade (ver Equação (2-28)), (Brownjohn et al., 1989).

A principal desvantagem do método PP é a dificuldade para identificar de forma confiável as frequências naturais do sistema por meio da seleção dos picos no espectro médio. Essa dificuldade deve-se, principalmente, à existência de modos próximos um do outro, que geralmente se apresenta em estruturas civis. Além disso, o ruído intrínseco no sinal que se apresenta no espectro dificulta ainda mais essa seleção de frequências naturais.

\subsubsection{Método de decomposição do domínio da frequência (FDD)}

As anteriores dificuldades expressas no método básico de seleção de picos fazem surgir uma nova técnica conhecida como a decomposição do domínio da frequência (Frequency Domain Decomposition-FDD) divulgada por Brincker, Zhang et al., (2000). A base deste método é a técnica matemática clássica conhecida como a decomposição de valores singulares (Singular Value Decomposition-SVD), a qual é aplicada diretamente à matriz de PSD da resposta para cada frequência discreta $i \omega_{j}$. 
$\left[S\left(i \omega_{j}\right)\right]_{N \times N}=\left[U\left(i \omega_{j}\right)\right]_{N \times N}\left[\Sigma\left(i \omega_{j}\right)\right]_{N \times N}\left[V\left(i \omega_{j}\right)\right]_{N \times N}^{H}$

Em que, $\boldsymbol{N}$, é o número de graus de liberdade medidos, $\boldsymbol{H}$, é a transposta conjugada, $U$ e $V$ são os vetores singulares de $S$, e $\Sigma$ é uma matriz diagonal que contém os valores singulares de $S$, da forma $\Sigma=\operatorname{diag}\left(\sigma_{1}, \sigma_{2} \ldots \sigma_{N}\right)$ com $\sigma_{1} \geq \sigma_{2} \geq \ldots \geq \sigma_{N}>0 . A$ técnica SVD encontra-se relacionada com a determinação dos valores e vetores próprios das matrizes $S^{H} S$ e $S S^{H}$. Os valores singulares de $S$ são iguais às raízes quadradas dos valores próprios $U_{j}$ de $S^{H} S$ e $S S^{H}$. As colunas de $U$ contém os vetores próprios unitários de $S S^{H}$ e as colunas de $V$ os vetores próprios unitários $S^{H} S$. As matrizes $S^{H} S$ e $S S^{H}$ são matrizes simétricas, portanto, os vetores próprios resultantes são ortogonais entre eles.

O uso da técnica SVD tem sido usado originalmente na área de controle robusto multivariável para avaliação do ganho de um sistema MIMO (Ringwood, 1995), no qual se faz uma decomposição em valores singulares da matriz de transferência. Portanto, aplicar essa técnica na matriz de densidade espectral terá como objetivo, igualmente, encontrar o máximo ganho do sistema, considerando que a matriz de densidade espectral seja proporcional à matriz de transferência. Essa proporcionalidade é valida quando o sistema é excitado por um ruído branco como é mostrado na Equação (2-19) e que se mostra aqui na forma matricial.

$$
\begin{aligned}
& \left.S_{x_{k} X_{j}}(i \omega)=\sigma^{2}\left[H_{x_{k} f_{r}}(i \omega)\right]^{*}[I] \cdot \mid H_{x_{j} f_{s}}(i \omega)\right]^{T} \\
& \text { O ganho para um sistema MIMO vem dado pela seguinte relação. } \\
& G(i \omega)=\frac{\left\|\left\{X_{k}(i \omega)\right\}\right\|}{\left\|\left\{F_{r}(i \omega)\right\}\right\|}=\frac{\left\|\left[H_{x_{k} f_{r}}(i \omega)\right]\left\{F_{r}(i \omega)\right\}\right\|}{\left\|\left\{F_{r}(i \omega)\right\}\right\|}
\end{aligned}
$$

Em que, $\|$.$\| é a norma Euclidiana. Assim, supondo que a densidade espectral seja$ equivalente à função de transferência o ganho do sistema se pode representar, como.

$$
G(i \omega)=\frac{\left\|\left\{X_{k}(i \omega)\right\}\right\|}{\left\|\left\{F_{r}(i \omega)\right\}\right\|}=\frac{\left.\| S_{x_{k} x_{j}}(i \omega)\right]\left\{F_{r}(i \omega)\right\} \|}{\left\|\left\{F_{r}(i \omega)\right\}\right\|}
$$

No caso, que $\left\{F_{r}(i)\right\}$ seja um vetor unitário o ganho fica como.

$$
\underset{\left\|\left\{F_{r}(i \omega)\right\}\right\|=1}{G(i \omega)}=\left\|\left[S_{x_{k} x_{j}}(i \omega)\right]\left\{F_{r}(i \omega)\right\}\right\|
$$


Então, pode-se mostrar que a norma Euclidiana elevada ao quadrado de multiplicar $\left[S_{x_{k} x_{j}}(i \omega)\right\rfloor$ pela primeira coluna da matriz de vetores singulares, $V(i \omega)$, é igual ao máximo valor singular de $\left[S_{x_{k} x_{j}}(i \omega)\right]$.

$$
\begin{aligned}
\left\|\left[S_{x_{k} x_{j}}(i \omega)\right]\{V(i \omega)\}_{1}\right\|^{2}= & \left.\left.\left.\left.\left(\left[S_{x_{k} x_{j}}(i \omega)\right]\right\} V(i \omega)\right\}_{1}\right)^{T}\left(\left[S_{x_{k} x_{j}}(i \omega)\right]\right\} V(i \omega)\right\}\right) \\
= & \{V(i \omega)\}_{1}^{T}\left(\left[S_{x_{k} x_{j}}(i \omega)\right]^{T}\left[S_{x_{k} x_{j}}(i \omega)\right]\{V(i \omega)\}_{1}\right) \\
& =\{V(i \omega)\}_{1}^{T} u_{1}(i \omega)\{V(i \omega)\}_{1} \\
\left\|\left[S_{x_{k} x_{j}}(i \omega)\right]\{V(i \omega)\}_{1}\right\|= & \sqrt{u_{1}(i \omega)}=\Sigma(i \omega)_{11}=\sigma_{1}(i \omega)
\end{aligned}
$$

$\mathrm{Na}$ anterior equação foram consideradas as seguintes relações:

$$
\begin{aligned}
& \left.\mid S_{x_{k} x_{j}}(i \omega)\right]^{T}\left|S_{x_{k} x_{j}}(i \omega)\right|\{V(i \omega)\}_{1}=u_{1}(i \omega)\{V(i \omega)\}_{1} \\
& \{V(i \omega)\}_{1}^{T}\{V(i \omega)\}_{1}=1
\end{aligned}
$$

Uma maneira intuitiva de se entender o anterior resultado é notando que $\sigma_{1}$ é o máximo ganho sobre um conjunto ortogonal de direções da entrada definido pelos vetores singulares à direita, $V(i \omega)$. Assim, o ganho máximo pode ser obtido quando o vetor de entrada unitário, $\left\{F_{r}(i \omega)\right\}$, é proporcional ou paralelo, $\{V(i \omega)\}_{1}$. Além disso, pode-se notar na parte direita da equação de resposta modal (Equação (2-34)), igual a, $\{\Phi\}_{j}^{T}\left\{f_{r}\right\}$, que, a máxima força de entrada para um vetor unitário $\left\{f_{r}\right\}$ é dada quando a entrada é paralela ao modo de vibração, portanto, pode-se concluir que os vetores singulares à direita, $\{V(i \omega)\}_{1}$, na ressonância serão proporcionais aos vetores modais, para alcançar o máximo ganho.

Dessa forma, na técnica FDD as frequências naturais podem ser avaliadas dos picos do gráfico de valores singulares, $\sigma_{1}(i \omega)$, ou máximos ganhos e as correspondentes formas modais podem ser obtidas dos vetores singulares, $\{V(i \omega)\}_{1}$, em cada frequência de ressonância, $i \omega_{l}$.

\subsubsection{Método melhorado do FDD}

A primeira geração de FDD pode só estimar frequências naturais e formas modais. A segunda geração conhecida como melhora do FDD ou EFDD (Enhanced Frequency Domain Decomposition) proposta por Brincker, Ventura et al., (2001), pode estabelecer também as taxas de amortecimento, através de aplicar uma IFFT aos valores singulares vizinhos da frequência natural, a qual é uma aproximação da função de correlação de um sistema SDF (ver Equação (2-29)). Esta aproximação também considera o fato que foi demostrado no método PP na qual as vizinhanças da frequência de ressonância podem ser modeladas 
como de um oscilador de um só grau de liberdade. No entanto, o tamanho dessa vizinhança é definido através de aplicar o critério de correlação modal (Modal Assurance Criterion-MAC) entre o vetor singular $\left\{V(i \omega)_{1}\right\}$ da frequência natural, $i \omega_{l}$, e os vetores singulares, $\boldsymbol{j}$, vizinhos. O critério de correlação modal pode assumir um valor entre 0 e 1 , no que um valor de 1 significa que os vetores são paralelos e 0 que são ortogonais, assim é possível determinar um limite mínimo para o valor do MAC e escolher a vizinhança nesse valor. O MAC é determinado a partir da seguinte relação (Allemang, 2003).

$$
M A C=\frac{\left\{V\left(i \omega_{l}\right)\right\}^{T}\left\{V\left(i \omega_{j}\right)\right\}}{\left(\left\{V\left(i \omega_{l}\right)\right\}^{T}\left\{V\left(i \omega_{l}\right)\right\}\right)\left(\left\{V\left(i \omega_{j}\right)\right\}^{T}\left\{V\left(i \omega_{j}\right)\right\}\right)}
$$

Depois de aplicar a IFFT aos valores singulares escolhidos dentro da vizinhança da frequência de ressonância $i \omega_{l}$ obtém-se uma função de correlação que corresponde à resposta transiente de um sistema SDF, na qual pode ser calculada a porcentagem de amortecimento pelo uso da técnica do decremento logarítmico (Chopra, 2001).

No método EFDD a estimação das formas modais também é melhorada considerando todos os vetores singulares dentro da vizinhança de cada frequência de ressonância, $i \omega_{l}$, ponderados pelo correspondente valor singular.

Finalmente, uma importante característica do método EFDD é que a partir do uso do indicador MAC, é possível identificar frequências de ressonância do sistema que estejam muito próximas sempre e quando elas sejam ortogonais.

\subsubsection{Métodos RD-FDD e RD-EFDD.}

Como já foi comentada na seção de estimação de funções de densidade espectral, uma possível aproximação das funções de densidades espectrais é aplicando a transformada de Fourier diretamente às funções RD (ver Equação (3-27)). Dessa forma, com o cálculo da densidade espectral por meio das funções RD podem ser aplicados às técnicas FDD ou EFDD, surgindo desta maneira os métodos RD-FDD ou RD-EFDD, como foram propostos por Rodrigues e Brincker, (2005). 


\subsubsection{Método decomposição do domínio da frequência-espaço (FSDD)}

Uma terceira geração do método FDD é a decomposição do domínio da frequênciaespaço (Frequency-Spatial Domain Decomposition-FSDD) desenvolvido por Zhang, Wang et al., (2010). O método faz uso da decomposição da matriz densidade espectral pela via de valores singulares, do mesmo modo que faz o método FDD. No entanto, o método EFDD avalia o amortecimento a partir de aplicar o método decremento logarítmico à função de correlação avaliada pela aplicação da IFFT aos valores singulares vizinhos da frequência de ressonância. Nesse aspecto, o cálculo da IFFT com um espectro truncado pode causar erros na estimação do amortecimento, principalmente quando existem frequências naturais muito próximas.

Desse modo, o método FSDD foi desenvolvido para eliminar o anterior tipo de problema do método EFDD. O método FSDD faz uma transformação linear da matriz espectral, pré-multiplicando e pós-multiplicando o vetor singular correspondente a uma frequência de ressonância, $i \omega_{l}$. Assim, fazendo uso das propriedades de ortogonalidade entre os modos de vibração a nova matriz espectral obtida terá um comportamento de um SDF.

A anterior propriedade da matriz de densidade espectral pode ser demostrado a partir de usar a Equação (3-38) na forma matricial e pré-multiplicando e pós-multiplicando por um vetor singular $\left\{V(i \omega)_{1}\right\}$ da frequência de ressonância $i \omega_{l}$ que é igual ao modo de vibração $\left\{\Phi_{l}\right\}$, assim.

$$
\begin{aligned}
& S_{x x}(i \omega)=\left\{\Phi_{l}\right\}_{N \times 1}^{T}\left[\Phi_{j}\right]_{N \times N}\left[\hat{H}^{*}(i \omega)\right]_{N \times N}\left[S_{f f}(i \omega)\right]_{N \times N}[\hat{H}(i \omega)]_{N \times N}{ }^{T}\left[\Phi_{j}\right]_{N \times N}{ }^{T}\left\{\Phi_{l}\right\}_{N \times 1} \\
& =\left\{\delta_{l, j}\right\}_{N \times 1}{ }^{T}\left[\hat{H}^{*}(i \omega)\right]_{N \times N}\left[S_{f f}(i \omega)\right]_{N \times N}[\hat{H}(i \omega)]_{N \times N}{ }^{T}\left\{\delta_{l, j}\right\}_{N \times 1} \quad \text { com } \quad \delta_{l, j}=\left\{\begin{array}{ll}
1 & l=j \\
0 & l \neq j
\end{array}\right\}
\end{aligned}
$$

O $\delta_{l, j}$ estabelece a propriedade de ortogonalidade dos modos de vibração, em que $\left\{\Phi_{l}\right\}^{T}\left\{\Phi_{j}\right\}=1$ se $l=j$ e $\left\{\Phi_{l}\right\}^{T}\left\{\Phi_{j}\right\}=0$ se $l \neq j$. Uma representação em termos de um somatório pode ser dada, como.

$S_{x_{k} \chi_{j}}(i \omega)=\sum_{r=1}^{N} \sum_{s=1}^{N} \hat{H}_{z_{l} f_{r}}{ }^{*}(i \omega) S_{f_{r} r_{s}}(i \omega) \hat{H}_{z_{l} f_{s}}(i \omega)$

Em que, os termos $\hat{H}_{z_{l} f_{r}}{ }^{*}\left(i \omega_{l}\right)$ e $\hat{H}_{z_{l} f_{s}}\left(i \omega_{l}\right)$ respondem a um modelo de um sistema de um só grau de liberdade na frequência de ressonância $i \omega_{l}$. Pode-se notar que o vetor singular correspondente a uma frequência de ressonância $i \omega_{l}$ atua nesse método como um filtro modal da matriz espectral. Então, a nova matriz espectral filtrada pode ser 
aplicada a IFFT para avaliar a função de correlação e avaliar a porcentagem de amortecimento modal, sem a necessidade do truncamento do espectro.

\subsubsection{Método baseado em transmissibilidade.}

Os diferentes métodos descritos anteriormente fazem uso da suposição de uma excitação de ruído branco. Com respeito a essa suposição surge uma aproximação para identificar parâmetros modais em OMA, baseado no conceito de transmissibilidade (Transmissibility Operational Modal Analysis-TOMA) como foi apresentado em Devriendt e Guillaume (2007). Nessa proposta para a análise modal baseada no conceito de transmissibilidade não se precisa que a força de excitação seja considerada como ruído branco. A força pode ser arbitraria como ruído colorido, varredura de senos ou impacto, etc, sempre que seja garantido que estas forças consigam excitar as frequências do sistema. No entanto, geralmente os pólos que são identificados das medições de transmissibilidade não correspondem com os pólos do sistema, porém, fazendo uma combinação de medições de transmissibilidade sob diferentes condições de carregamento é possível identificar parâmetros modais (Devriendt e Guillaume, 2008).

No Capítulo 2 foi demostrado que o limite da função de transmissibilidade quando i $\omega$ tende para um pólo do sistema, $\lambda_{l}$, converge à seguinte relação.

$$
\lim _{i \omega \rightarrow \lambda_{l}} T^{k}{ }_{{ }_{o} x_{j}}(i \omega)=\lim _{i \omega \rightarrow \lambda_{l}} \frac{X_{o}(i \omega)}{X_{j}(i \omega)}=\lim _{i \omega \rightarrow \lambda_{l}} \frac{H_{x_{o} f_{k}}(i \omega) F_{k}(i \omega)}{H_{x_{j} f_{k}}(i \omega) F_{k}(i \omega)}=\frac{\phi_{o l}}{\phi_{j l}}
$$

Em que, $\phi_{o l}$ e $\phi_{j l}$ são as componentes modais do modo I nos graus de liberdade $\boldsymbol{o}$ e $\boldsymbol{j}$, respectivamente. Assim, pode-se notar que o limite da transmissibilidade quando tende para um pólo $\lambda_{l}$ é independente da localização da excitação, $\boldsymbol{k}$, e do espectro da excitação. Consequentemente, estabelecer uma subtração de duas funções de transmissibilidade com os mesmos graus de liberdade de saída, mas com diferentes entradas $\boldsymbol{k}$ e $\boldsymbol{z}$, resultam.

$$
\lim _{i \omega \rightarrow \lambda_{l}}\left(T^{k}{ }_{\alpha_{o} x_{j}}(i \omega)-T^{2}{ }_{x_{o} x_{j}}(i \omega)\right)=\frac{\phi_{o l}}{\phi_{j l}}-\frac{\phi_{o l}}{\phi_{j l}}=0
$$

Isto significa que os pólos, $\lambda_{l}$, do sistema são zeros na função racional, $\Delta T^{k z}{ }_{x_{o} x_{j}}(i \omega)=\left(T^{k}{ }_{x_{o} x_{j}}(i \omega)-T^{z}{ }_{x_{o} x_{j}}(i \omega)\right)$, e, portanto, serão pólos na inversa. 
$\Delta^{-1} T^{k z}{ }_{x_{o} x_{j}}(i \omega)=\frac{1}{\Delta T^{k z}{ }_{x_{o} x_{j}}(i \omega)}=\frac{1}{\left(T^{k}{ }_{x_{o} x_{j}}(i \omega)-T^{z}{ }_{x_{o} x_{j}}(i \omega)\right)}$

Note que a função racional $\Delta T_{x_{o} x_{j}}^{k z}$ pode ser reescrita em termos de numeradores e denominadores das funções de transferência, como.

$$
\begin{aligned}
& \Delta^{-1} T^{k x_{x_{o} x_{j}}}(i \omega)=\frac{1}{\Delta T^{k z_{x_{o}} x_{j}}(i \omega)}=\frac{1}{\left(\frac{H_{x_{o} f_{k}}(i \omega)}{H_{x_{j} f_{k}}(i \omega)}-\frac{H_{x_{o} f_{z}}(i \omega)}{H_{x_{j} f_{z}}(i \omega)}\right)} \\
& =\frac{1}{\left(\frac{N_{o k}(i \omega) D_{j k}(i \omega)}{N_{j k}(i \omega) D_{o k}(i \omega)}-\frac{N_{o z}(i \omega) D_{j z}(i \omega)}{N_{j z}(i \omega) D_{o z}(i \omega)}\right)} \\
& =\frac{N_{j k}(i \omega) D_{o k}(i \omega) N_{j z}(i \omega) D_{o z}(i \omega)}{N_{o k}(i \omega) D_{j k}(i \omega) N_{j z}(i \omega) D_{o z}(i \omega)-N_{j k}(i \omega) D_{o k}(i \omega) N_{o z}(i \omega) D_{j z}(i \omega)}
\end{aligned}
$$

Assim, é notável que os pólos da função racional, podem exceder os pólos do sistema, devido aos zeros $N_{o k}, N_{j z}, N_{j k}, N_{o z}$ conteúdos no denominador. Portanto, em geral só um subconjunto dos pólos de $\Delta T_{x_{o} x_{j}}^{k z}$ corresponderão aos pólos do sistema.

Para o anterior problema, em Devriendt e Guillaume (2008) foi dada uma solução numérica que consiste em estabelecer uma matriz quadrada de seis medições de transmissibilidade para três combinações de carga diferentes, $\mathbf{k}, \boldsymbol{z}$, e $\boldsymbol{v}$ usando três graus de liberdade, $\boldsymbol{o}, \boldsymbol{j}$, e $\boldsymbol{h}$, assim.

$$
T(i \omega)=\left[\begin{array}{ccc}
T^{k}{ }_{{ }_{o} x_{j}}(i \omega) & T^{2}{ }_{x_{o} x_{j}}(i \omega) & T^{v}{ }_{x_{o} x_{j}}(i \omega) \\
T^{k}{ }_{{ }_{x_{h} x_{j}}}(i \omega) & T^{2}{ }_{x_{x_{h} x_{j}}}(i \omega) & T^{{ }^{v}{ }_{x_{h} x_{j}}}(i \omega) \\
1 & 1 & 1
\end{array}\right]
$$

Então, considerando o fato que as funções de transmissibilidade convergem ao mesmo valor quando estão nos pólos do sistema, todas as colunas da matriz $T(i \omega)$ serão linearmente dependentes. Portanto, o posto da matriz será igual a 1 nos pólos do sistema. Uma técnica para se avaliar o posto da matriz é a avaliação dos valores singulares da matriz, a quantidade de valores singulares da matriz diferentes de zero é igual ao posto. $O$ anterior significa que o segundo valor singular, $\sigma_{2}$, converge para zero em i $\omega=\lambda_{1}$. Assim, os picos do gráfico da inversa dos segundos valores singulares $1 / \sigma_{2}(i \omega)$ coincidem com as frequências de ressonância.

A aproximação descrita anteriormente considera só uma entrada ao sistema em cada uma das funções de transmissibilidade. Porém, na maioria das aplicações existe uma situação de múltiplas entradas. Nessa situação, a partir do uso da Equação (3-33) a transmissibilidade se representa, como. 


$$
T^{F_{x_{0}} x_{j}}(i \omega)=\frac{X_{o}(i \omega)}{X_{j}(i \omega)}=\frac{\phi_{01} \sum_{r=1}^{N} \hat{H}_{z_{1} f_{r}}(i \omega) F_{r}(i \omega)+\phi_{o 2} \sum_{r=1}^{N} \hat{H}_{z_{2} f_{r}}(i \omega) F_{r}(i \omega)+. .+\phi_{o N} \sum_{r=1}^{N} \hat{H}_{z_{N} f_{r}}(i \omega) F_{r}(i \omega)}{\phi_{j 1} \sum_{r=1}^{N} \hat{H}_{z_{1} f_{r}}(i \omega) F_{r}(i \omega)+\phi_{j 2} \sum_{r=1}^{N} \hat{H}_{z_{2} f_{r}}(i \omega) F_{r}(i \omega)+. .+\phi_{j N} \sum_{r=1}^{N} \hat{H}_{z_{N} f_{r}}(i \omega) F_{r}(i \omega)}
$$

O limite da função de transmissibilidade para múltiplas entradas quando $i \omega$ tende para um pólo do sistema $\lambda_{l}$ é o mesmo valor como para uma só entrada, dada por.

$$
\begin{aligned}
\lim _{i \omega \rightarrow \lambda_{l}} T^{F_{r_{x_{0}} x_{j}}}(i \omega) & =\lim _{i \omega \rightarrow \lambda_{l}} \frac{\phi_{o 1} \sum_{r=1}^{N} \hat{H}_{z_{1} f_{r}}(i \omega) F_{r}(i \omega)+\phi_{o 2} \sum_{r=1}^{N} \hat{H}_{z_{z_{1}} f_{r}}(i \omega) F_{r}(i \omega)+\phi_{j 2} \sum_{r=1}^{N} \hat{H}_{z_{2} f_{r}}(i \omega) F_{r}(i \omega)+. .+\phi_{o N} \sum_{r=1}^{N} \hat{H}_{z_{N} f_{r}}(i \omega)+. .+\phi_{j N} \sum_{r=1}^{N} \hat{H}_{z_{N} f_{r}}(i \omega) F_{r}(i \omega)}{} \\
& =\frac{\phi_{o l} \sum_{r=1}^{N} \hat{H}_{z_{1} f_{r}}(i \omega) F_{r}(i \omega)}{\phi_{j l} \sum_{r=1}^{N} \hat{H}_{z_{1} f_{r}}(i \omega) F_{r}(i \omega)} \\
& =\frac{\phi_{o l}}{\phi_{j l}}
\end{aligned}
$$

O anterior conclui que o procedimento é ainda aplicável para múltiplas entradas, $F_{r}$, ao sistema.

Baseados na ideia apresentada anteriormente têm sido propostos diferentes aproximações para a análise modal operacional, considerando diferentes funções de transmissibilidade determinadas a partir de diferentes condições de carga. Devriendt, De Sitter et al. (2010) propuseram uma aproximação usando uma relação entre as funções de transmissibilidade multivariável (ver Equação (2-62)) e as "pseudo-funções de transmissibilidade escalar". Neste trabalho foi demostrado que as funções de transmissibilidade multivariável não convergem para a mesma relação de modos de vibração para diferentes condições de carga e, portanto, elas não podem ser usadas para identificação dos pólos do sistema. Dessa forma, foi encontrada uma relação entre as funções de transmissibilidade multivariável e as funções de transmissibilidade escalar, resultando em uma solução ao problema.

Devriendt et al. (2013) propuseram um método que combina em uma única matriz todas as funções de transmissibilidade advindo de diferentes condições de carga. Neste trabalho a inversa generalizada da matriz foi usada para identificação dos pólos do sistema, desse modo foi encontrado uma redução do risco de encontrar pólos não pertencente ao sistema em comparação com o uso das funções de subtração inversa entre funções de transmissibilidade. 
Weijtjens, Lataire, et al. (2014) propuseram obter vetores próprios e valores próprios através de uma estimação paramétrica a partir de funções de transmissibilidade associadas com diferentes condições de carga. Neste trabalho foi proposto que os parâmetros modais podem ser estimados usando os seguintes passos: 1) obter as respostas do sistema para diferentes condições de carga, 2) estimar os parâmetros polinomiais para diferentes funções de transmissibilidade relacionadas com diferentes condições de carga, e 3) resolverem um problema de valores próprios com os parâmetros polinomiais.

Recentemente Weijtjens, De Sitter, et al. (2014) desenvolveram o conceito de funções de transmissibilidade variando no tempo com o fim de lidar com a continua mudança das condições de carga na estrutura. Dessa maneira, as funções de transmissibilidade variando no tempo podem ser implementadas, de tal forma que o método seja menos dependente do usuário para identificar as diferentes condições de carga.

\subsubsection{Método baseado em transmissibilidade escalar com densidade espectral (PSDT).}

Uma limitação das anteriores metodologias baseada em transmissibilidade é que devem ser obtidas durante a experimentação diferentes condições de carga, as quais podem ser difíceis de serem obtidas em grandes estruturas até mesmo com o uso de excitação artificial em combinação com vibração do ambiente (vento, tráfego, sismo, etc). Uma solução alternativa devida à anterior dificuldade foi apresentada em Yan e Ren (2012) mediante o uso de transmissibilidade escalar com densidade espectral de potência (Power Spectrum Density Transmissibility-PSDT). Nesta aproximação é possível obter os parâmetros modais do sistema por meio da combinação de diferentes PSDT com diferentes referências de saída para uma só condição de carga.

A função de transmissibilidade escalar com o uso de funções de densidade espectral foi definida no Capítulo 2 na Equação (2-58), como.

$T^{k}{ }_{x_{o} x_{j}}(i \omega)=\frac{S_{x_{o} x_{k}}(i \omega)}{S_{x_{j} x_{k}}(i \omega)}=\frac{\sum_{r=1}^{N} \sum_{s=1}^{N} H^{*}{ }_{x_{o} f_{r}}(i \omega) \cdot H_{x_{k} f_{s}}(i \omega) \cdot S_{f_{r} f_{s}}(i \omega)}{\sum_{r=1}^{N} \sum_{s=1}^{N} H^{*}{ }_{x_{j} f_{r}}(i \omega) \cdot H_{x_{k} f_{s}}(i \omega) \cdot S_{f_{r} f_{s}}(i \omega)}$

Em que, $T_{{ }_{x_{0} x_{j}}}^{k}(i \omega)$ define uma relação das saídas $x_{o}$ e $x_{j}$ com referência à saída $x_{k}$. Como tem sido comentado anteriormente, na transmissibilidade com densidade 
espectral o limite quando $i \omega$ tende para um pólo, $\lambda_{l}$, do sistema, converge a uma relação de modos de vibração. Isso pode ser provado a partir de usar a Equação (3-37), como.

$$
\begin{aligned}
& \lim _{i \omega \rightarrow \lambda_{l}} T^{k}{ }_{x_{0} x_{j}}(i \omega)=\lim _{i \omega \rightarrow \lambda_{l}} \frac{S_{x_{x_{1}} x_{k}}(i \omega)}{S_{x_{j} x_{k}}(i \omega)}
\end{aligned}
$$

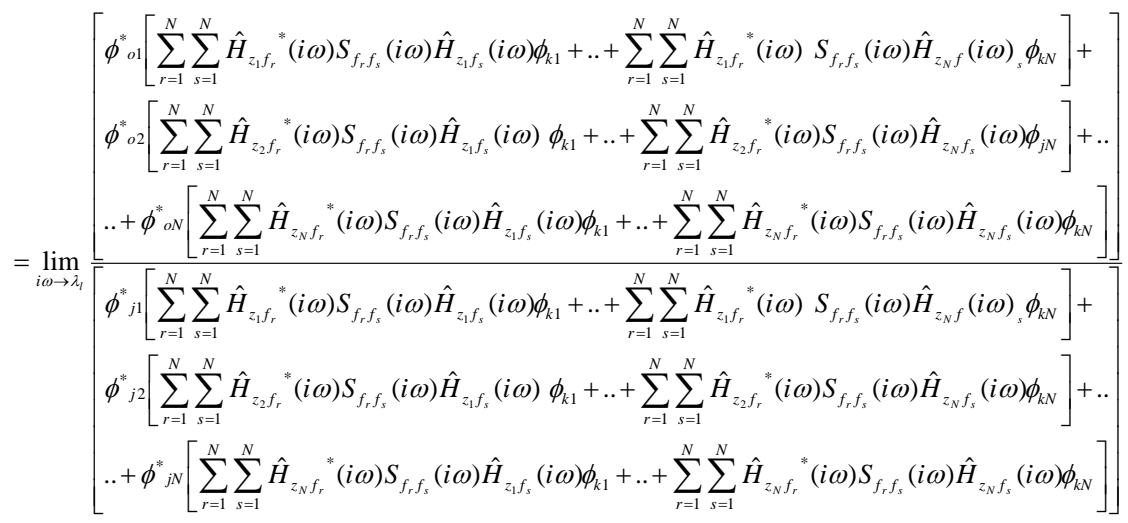

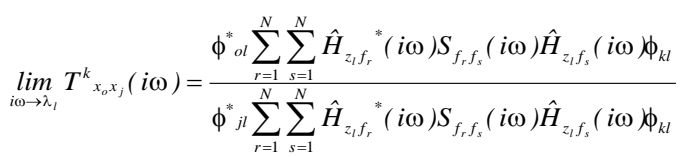

$$
\begin{aligned}
& =\frac{\phi^{*}{ }_{o l}}{\phi^{*}{ }_{j l}}
\end{aligned}
$$

Logo, as PSDTs são independentes da posição e do espectro da excitação quando $i \omega=\lambda_{1}$. Do mesmo modo, pode-se estabelecer uma escalar PSDT com diferentes referências da saída $\boldsymbol{z}$ (Transferring output), na qual se tende ao mesmo valor quando $i \omega=\lambda_{1}$

$$
\lim _{i \omega \rightarrow \lambda_{l}} T^{2}{ }_{x_{o} x_{j}}(i \omega)=\lim _{i \omega \rightarrow \lambda_{l}} \frac{S_{x_{x} x_{z}}(i \omega)}{S_{x_{j} x_{z}}(i \omega)}=\frac{\phi^{*}{ }_{o l}}{\phi^{*}{ }_{j l}}
$$

Então, estabelecendo uma subtração de duas escalares PSDTs com os mesmos graus de liberdade de relação, mas com uma referência de saída diferente, tem-se que.

$$
\lim _{i \omega \rightarrow \lambda_{l}} \Delta T^{k z}{ }_{x_{o} x_{j}}(i \omega)=\lim _{i \omega \rightarrow \lambda_{l}}\left(T^{k}{ }_{x_{0} x_{j}}(i \omega)-T^{z}{ }_{x_{o} x_{j}}(i \omega)\right)=\frac{\phi^{*}{ }_{o l}}{\phi^{*}{ }_{j l}}-\frac{\phi^{*}{ }_{o l}}{\phi^{*}{ }_{j l}}=0
$$

Isso mostra que os pólos do sistema são zeros na função racional $\Delta T_{x_{o} x_{j}}^{k z}(i \omega)$ (Transmissibility Substraction Function-TSF), os quais serão pólos na função inversa, $\Delta^{-1} T_{x_{o} x_{j}}^{k z}(i \omega)$ (Inverse Transmissibility Substraction Function-ITSF). Assim, as frequências naturais amortecidas do sistema podem ser determinadas por uma simples observação dos picos das funções ITSF. Em Yan e Ren (2012) é adoptado a média normalizada das ITSF 
(Average Normalized Inverse Transmissibility Substraction Function-ANITSF) para a identificação das frequências naturais. Assim, como as ANPSDs do método PP, as ANITSF são definidas como uma média das normalizadas ITSFs, $N \Delta^{-1} T_{{ }_{x_{0}} x_{j}}^{k z}(i \omega)$, como.

$\operatorname{ANITSF}(i \omega)=\frac{1}{N} \sum_{k=1}^{N} N \Delta^{-1} T^{k z}{ }_{x_{0} x_{j}}(i \omega)$

Em que, $\boldsymbol{N}$ é número de $N \Delta^{-1} T^{k z}{ }_{x_{0} x_{j}}(\mathrm{i} \omega)$ escolhidas. As ITSFs normalizadas são obtidas, dividendo as ITSFs pelas somas de suas respectivas amplitudes, como.

$$
N \Delta^{-1} T^{k z}{ }_{x_{o} x_{j}}(i \omega)=\frac{\Delta^{-1} T^{k z}{ }_{x_{o} x_{j}}(i \omega)}{\sum_{j=1}^{L} \Delta^{-1} T^{k z}{ }_{x_{o} x_{j}}(i \omega)}
$$

Os modos de vibração nesta metodologia são avaliados da mesma forma como foi descrita no método PP.

\subsection{MÉTODO PARAMÉTRICO NO DOMÍNIO DA FREQUÊNCIA.}

Métodos de identificação baseados em um ajuste de um modelo da densidade espectral da saída podem ser usados no domínio da frequência. Assim, a partir deste modelo ajustado parâmetros modais podem ser extraídos do sistema medido. O ajuste de um modelo teórico a partir de dados experimentais é um problema de optimização baseado em uma função de custo, que pode ser resolvida a través do método dos mínimos quadrados (Linear Least Square) ou com o estimador Maximum Likelihood (Guillaume, Hermans e Van Der Auweraer, 1999; Magalhães, 2013). Um estudo completo do uso de diferentes modelos e procedimentos de ajuste foi dado em Cauberghe (2004), incluindo também um estudo de outra classe de métodos (Realization Algorithms) que usam modelos de espaço de estado no domínio da frequência (Frequency domain state space models).

Na presente seção é descrito com detalhe somente um método paramétrico no domínio da frequência: the poly-Least Squares Complex Frequency domain method ( $p$-LSCF) (Peeters et al., 2004). Esse método também conhecido como PolyMAX foi selecionado devido ao bom desempenho que tem sido mostrado na literatura, principalmente na aplicação em estruturas de engenharia civil (Magalhães e Cunha, 2011). 


\section{MÉTODO P-LSCF.}

O método p-LSCF foi primeiro desenvolvido para identificar parâmetros dinâmicos a partir da função de resposta em frequência (Peeters et al., 2004). No entanto, como já foi demonstrado no Capítulo 1 existe uma similaridade entre a função de transferência e a densidade espectral quando existe uma excitação do tipo ruído branco. Portanto, um ajuste do método pode ser realizado para ser aplicado na análise modal operacional (Peeters e Van Der Auweraer, 2005).

O método p-LSCF começa por descrever a matriz de densidade espectral da saída usando o modelo rigth matrix-fraction no tempo discreto. Desta forma, a matriz de densidade espectral avaliada em cada frequência $i \omega_{j}$ é modelada por.

$$
\left[S_{y y}\left(i \omega_{j}\right)\right]_{l \times m}=[B][A]^{-1}=\left[\sum_{r=0}^{p}\left[B_{r}\right] e^{i \omega_{j} \cdot \Delta t \cdot r}\right]_{l \times m}\left[\sum_{r=0}^{p}\left[A_{r} e^{i \omega_{j} \cdot \Delta t \cdot r}\right]_{m \times m}^{-1}\right.
$$

Em que B e A são as matrizes com os parâmetros do modelo, $p$ é a ordem dos polinômios e $\Delta t$ é o tempo de amostragem usado para medir a resposta da estrutura. $O$ número de linhas da densidade espectral é igual ao número de graus de liberdade medidos na estrutura (I). Cada linha com $m$ colunas do modelo rigth matrix-fraction pode ser escrito como.

$$
\left\{S_{y y}\left(i \omega_{j}\right)\right\}_{o \times m}=[B][A]^{-1}=\left\{\sum_{r=0}^{p}\left[B_{r}\right] e^{i \omega_{j} \cdot \Delta t \cdot r}\right\}_{o \times m}\left[\sum_{r=0}^{p}\left[A_{r}\right]^{i \omega_{j} \cdot \Delta t \cdot r}\right]^{-1} \quad o=1,2, \ldots l
$$

O objetivo do método p-LSCF é estabelecer os parâmetros do modelo, as matrizes $B_{r}$ e $A_{r}$, as quais minimizam a diferença entre a matriz de densidade espectral surgida a partir dos dados de vibração (representada por $S_{y y}\left(i \omega_{j}\right)$ ) e o espectro teórico dado na Equação (3-63).

$$
\left[E^{N L S}\left(i \omega_{j}\right)\right]_{l \times m}=\left[\sum_{r=0}^{p} B_{r} e^{i \omega_{j} \cdot \Delta t \cdot r}\right]_{l \times m}\left[\sum_{r=0}^{p} A_{r} e^{i \omega_{j} \cdot \Delta t \cdot r}\right]_{m \times m}^{-1}-\left[S_{y y}\left(i \omega_{j}\right)\right]_{l \times m}
$$

A solução da Equação (3-65) gera um problema de minimização não linear (nonlinear least square). Para evitar está complexidade, uma equação alternativa do erro é formulada pela multiplicação de A em ambos os membros da Equação (3-65). 
$\left[E^{L S}\left(i \omega_{j}\right)\right]_{l \times m}=\left[\sum_{r=0}^{p} B_{r} e^{i \omega_{j} \cdot \Delta t \cdot r}\right]_{l \times m}-\left[S_{y y}\left(i \omega_{j}\right)\right]_{l \times m}\left[\sum_{r=0}^{p} A_{r} e^{i \omega_{j} \cdot \Delta t \cdot r}\right]_{m \times m}$

Nesta equação as funções polinomiais básicas podem ser organizadas em um vetor linha com $(p+1)$ componentes, assim.

$$
\left\{\Omega\left(i \omega_{j}\right)\right\}_{1 \times(p+1)}=\left[\begin{array}{llll}
\Omega_{0}\left(i \omega_{j}\right) & \Omega_{1}\left(i \omega_{j}\right) & \ldots & \Omega_{p}\left(i \omega_{j}\right)
\end{array}\right]_{1 \times(p+1)}=\left[\begin{array}{llll}
e^{i \omega_{j} \cdot \Delta t \cdot 0} & e^{i \omega_{j} \cdot \Delta t \cdot 1} & \ldots & e^{i \omega_{j} \cdot \Delta t \cdot p}
\end{array}\right]_{\times(p+1)}
$$

Desta forma uma linha $\boldsymbol{o}$ da matriz erro $E^{L S}\left(i \omega_{j}\right)$ pode ser calculada com a seguinte equação.

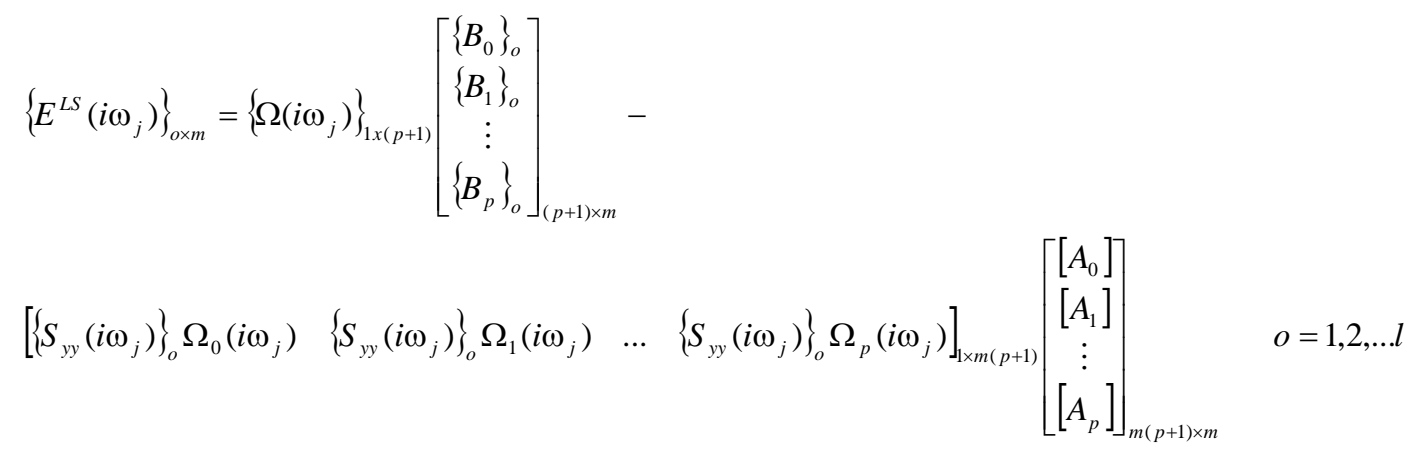

Em que $\left\{B_{0}\right\}_{o}$ representa uma linha da matriz $B,\left\{S_{y y}\left(i \omega_{j}\right)\right\}_{o}$ representa uma linha da matriz $S_{y y}\left(i \omega_{j}\right)$ e $[A]$ é a matriz A. Uma representação de forma mais generalizada do erro para todas as frequências, entre os valores $\omega_{1}$ até $\omega_{n_{f}}$ pode ser dada definindo as seguinte relações.

$$
\left[E_{o}\left(\beta_{o}, \alpha\right)\right]_{n f \times m}=\left[\begin{array}{ll}
X_{o} & Y_{o}
\end{array}\right]\left[\begin{array}{c}
\beta_{o} \\
\alpha
\end{array}\right]
$$

Com

$\beta_{o}=\left[\begin{array}{c}\left\{B_{0}\right\}_{o} \\ \left\{B_{1}\right\}_{o} \\ \vdots \\ \left\{B_{p}\right\}_{o}\end{array}\right]_{(p+1) \times m} \quad o=1,2, \ldots l$ e $\alpha=\left[\begin{array}{c}{\left[A_{0}\right]} \\ {\left[A_{1}\right]} \\ \vdots \\ {\left[A_{p}\right]}\end{array}\right]_{m(p+1) \times m}$ 


$$
\begin{aligned}
X_{o} & =\left[\begin{array}{c}
\Omega_{0}\left(i \omega_{1}\right) \\
\Omega_{0}\left(i \omega_{2}\right) \\
\vdots \\
\Omega_{0}\left(i \omega_{n f}\right)
\end{array}\right]_{n f \times(p+1)} \quad o=1,2, \ldots l \\
Y_{o} & =\left[\begin{array}{cccc}
\left\{S_{y y}\left(i \omega_{j}\right)\right\}_{o} \Omega_{0}\left(i \omega_{1}\right) & \left\{S_{y y}\left(i \omega_{j}\right)\right\}_{o} \Omega_{1}\left(i \omega_{1}\right) & \ldots & \left\{S_{y y}\left(i \omega_{j}\right)\right\}_{o} \Omega_{p}\left(i \omega_{1}\right) \\
\left\{S_{y y}\left(i \omega_{j}\right)\right\}_{o} \Omega_{0}\left(i \omega_{2}\right) & \left\{S_{y y}\left(i \omega_{j}\right)\right\}_{o} \Omega_{1}\left(i \omega_{2}\right) & \ldots & \left\{S_{y y}\left(i \omega_{j}\right)\right\}_{o} \Omega_{p}\left(i \omega_{2}\right) \\
\vdots & & & \\
\left\{S_{y y}\left(i \omega_{j}\right)\right\}_{o} \Omega_{0}\left(i \omega_{n f}\right) & \left\{S_{y y}\left(i \omega_{j}\right)\right\}_{o} \Omega_{1}\left(i \omega_{n f}\right) & \ldots & \left\{S_{y y}\left(i \omega_{j}\right)\right\}_{o} \Omega_{p}\left(i \omega_{n f}\right)
\end{array}\right]_{n f \times m(p+1)} \quad o=1,2, \ldots l
\end{aligned}
$$

Os parâmetros do modelo podem ser avaliados usando uma função de custo obtida pela soma de todos os erros da matriz $E_{o}\left(\beta_{o}, \alpha\right)$ elevados ao quadrado, para todas as frequências $\omega_{1}$ até $\omega_{n_{f}}$. No entanto, o método usa somente uma soma dos elementos da diagonal da matriz de erros ao quadrado como função de custo da seguinte forma.

$$
\begin{aligned}
& \varepsilon\left(\beta_{o}, \alpha\right)=\sum_{o=1}^{l} \operatorname{tr}\left(\left[E_{o}\left(\beta_{o}, \alpha\right)\right]^{H}\left[E_{o}\left(\beta_{o}, \alpha\right)\right]\right)=\sum_{o=1}^{l} \operatorname{tr}\left(\left[\begin{array}{ll}
\beta_{o}{ }^{T} & \left.\alpha^{T}\left[\begin{array}{c}
X_{o}^{H} \\
Y_{o}^{H}
\end{array}\right]\left[\begin{array}{ll}
X_{o} & Y_{o}
\end{array}\right]\left[\begin{array}{c}
\beta_{o} \\
\alpha
\end{array}\right]\right) \\
\varepsilon\left(\beta_{o}, \alpha\right)=\sum_{o=1}^{l} \operatorname{tr}\left(\left[\beta_{o}{ }^{T}\right.\right. & \left.\alpha^{T}\left[\begin{array}{cc}
R_{o} & S_{o} \\
S_{o}^{T} & T_{o}
\end{array}\right]\left[\begin{array}{c}
\beta_{o} \\
\alpha
\end{array}\right]\right) \quad \operatorname{Com} \quad R_{o}=\operatorname{Re}\left(X_{o}^{H} \cdot X_{o}\right) \quad ; \quad S_{o}=\operatorname{Re}\left(X_{o}^{H} \cdot Y_{o}\right) \\
T_{o}=\operatorname{Re}\left(Y_{o}^{H} \cdot Y_{o}\right)
\end{array}\right.\right.
\end{aligned}
$$

Na Equação (3-71) os termos $R_{o}, S_{o}$ e $T_{o}$ é selecionada somente a parte real devido a que é suposto que as matrizes a ser determinadas $B_{r}$ e $A_{r}$ contêm só valores reais. $O$ mínimo valor da função de custo é determinado pela derivação da função com respeito às variáveis desconhecidas (elementos das matrizes $B_{r}$ e $A_{r}$ ) e igualando ao zero. $\frac{\partial \varepsilon\left(\beta_{o}, \alpha\right)}{\partial \beta_{o}}=2\left(R_{o} \beta_{o}+S_{o} \alpha\right)=0 \quad o=1,2, \ldots l$

$\frac{\partial \varepsilon\left(\beta_{o}, \alpha\right)}{\partial \alpha_{o}}=2 \sum_{o=1}^{l}\left(S_{o}^{T} \beta_{o}+T_{o} \alpha\right)=0$

Com o fim de avaliar as variáveis desconhecidas pode-se primeiro despejar a variável $\beta_{o}$ na Equação (3-72).

$$
2\left(R_{o} \beta_{o}+S_{o} \alpha\right)=0 \Leftrightarrow \beta_{o}=-R_{o}^{-1} S_{o} \alpha
$$

E consequentemente a matriz $\alpha$ pode ser resolvida na Equação (3-73) pela substituição de $\beta_{o}$. 
$2 \sum_{o=1}^{l}\left(T_{o}-S_{o}^{T} R_{o}^{-1} S_{o}\right) \alpha=0 \Leftrightarrow M \alpha=0$

Em que, o tamanho da matriz $M$ é igual a $m(p+1) \times m(p+1)$, a qual pode ser calculada a partir da densidade espectral de potência dos dados de resposta. Com o objetivo de evitar uma solução trivial da Equação (3-75), uma restrição deve ser imposta. Esta restrição pode ser realizada impondo que alguns valores da matriz $\alpha$ sejam iguais a 1.Desta forma, 0 algoritmo estabelece igualar os valores da matriz $\left[A_{0}\right]$ como uma matriz identidade. Assim, a solução do sistema fica da seguinte forma.

$$
\begin{aligned}
& M \alpha=0 \Leftrightarrow\left[\begin{array}{ll}
{\left[M_{a a}\right]} & {\left[M_{a b}\right]} \\
{\left[M_{b a}\right]} & {\left[M_{b b}\right]}
\end{array}\right]\left[\begin{array}{c}
{[I]} \\
{\left[A_{1}\right]} \\
\vdots \\
{\left[A_{p}\right]}
\end{array}\right]=\left[\begin{array}{l}
0 \\
0 \\
0 \\
0
\end{array}\right] \\
& {\left[M_{b b}\right] \alpha_{b}=-\left[M_{b a}\right] \Leftrightarrow \alpha_{b}=-\left[M_{b b}\right]^{-1}\left[M_{b a}\right]} \\
& \Rightarrow \alpha=A=\left[\begin{array}{c}
{[I]} \\
-\left[M_{b b}\right]^{-1}\left[M_{b a}\right]
\end{array}\right]
\end{aligned}
$$

Em que, $[I]$ é do tamanho $m \times m,\left[M_{b b}\right]=M(m+1: m(p+1), m+1: m(p+1))$, $\left[M_{b a}\right]=M(m+1: m(p+1), 1: m)$. Uma vez as matrizes $\mathrm{A}_{\mathrm{r}}$ são determinadas, a matriz $\mathrm{B}_{\mathrm{r}}$ pode ser calculada usando a Equação (3-74).

A identificação dos parâmetros modais no método p-LSCF deve ser realizada através da avaliação das raízes dos polinômios formados com os coeficientes das matrizes $A_{r}$ ou $\alpha$ . No entanto, uma forma mais conveniente é representar os coeficientes dos polinômios na forma de uma matrix "companion", dessa forma os autovalores da matriz companion são as raízes polinomiais. A matriz companion é construída usando a seguinte expressão.

$$
A c o=\left[\begin{array}{ccccc}
-\left[A_{p}\right]^{-1}\left[A_{p-1}\right] & -\left[A_{p}\right]^{-1}\left[A_{p-2}\right] & \cdots & -\left[A_{p}\right]^{-1}\left[A_{1}\right] & -\left[A_{p}\right]^{-1}\left[A_{0}\right] \\
{[I]} & 0 & \cdots & 0 & 0 \\
\vdots & \vdots & \ddots & \vdots & \vdots \\
0 & 0 & \cdots & {[I]} & 0
\end{array}\right]
$$

Assim, o problema de autovalores é definido pela seguinte equação.

$$
\left([\text { Aco }]-u_{i}\right)[\Psi]=0
$$

A solução do problema de autovalores provê $\boldsymbol{m} * \boldsymbol{p}$ autovalores organizados em pares complexos conjugados. Os autovalores devem ser convertidos ao tempo continuo para 
poder estimar as frequências naturais e as taxas de amortecimento. As equações são dadas como.

$\lambda_{i}=\frac{\ln \left(\mu_{i}\right)}{\Delta t}, \xi_{i}=\frac{-\operatorname{Re}\left(\lambda_{i}\right)}{\left|\lambda_{i}\right|}, \omega_{d i}=\left|\lambda_{i}\right|$

Em que, $\xi_{i}, \omega_{d i}$ são as taxas de amortecimento e as frequências naturais amortecidas, respectivamente. Finalmente, os modos de vibração são estimados pelos componentes dos modos que podem ser observados, dados por.

$[\Phi]=\left[C_{c}\right][\Psi]$

Com

$\left[C_{c}\right]=\left[B_{p-1}-B_{p} A_{p}^{-1} A_{p-1} \quad \cdots \quad B_{0}-B_{p} A_{p}^{-1} A_{0}\right]$

Nesta metodologia não é possível estimar com antecipação qual deve ser a ordem correto do modelo. É por isso, que deve ser realizado um procedimento de repetição da análise através de incrementar a ordem do modelo. Este procedimento é chamado como diagrama de estabilidade. Para cada ordem do modelo, os parâmetros modais de frequências naturais e taxas de amortecimento devem ser calculados. Desta forma, deve ser analisada a variação desses parâmetros entre modelos com ordem consecutiva. Uma mínima variação entre modelos para cada um desses parâmetros pode definir a ordem correta que deve ser selecionada.

\subsection{CONSIDERAÇÕES FINAIS}

Apresentou-se uma revisão detalhada de diferentes métodos e técnicas existentes na análise modal operacional no domínio da frequência, na qual foram abordadas metodologias de identificação tradicionais e os métodos recentes baseados em transmissibilidade. Dessa forma, é possível notar atualmente uma abordagem diferente da análise modal operacional, na qual já não é mais considerada uma das mais importantes suposições da análise, como é a hipótese da entrada ao sistema se definir como ruído branco, com um espectro constante. Dita suposição é usada pela maioria dos métodos apresentados (PP, FDD, EFDD, RD-FDD, RD-EFDD, FSDD e p-LSCF). Novas metodologias têm sido propostas não baseadas nessa suposição, tais como os métodos que usam transmissibilidade. 
As diferentes metodologias tratadas nesta revisão têm como base a análise de sinais com o uso da transformada de Fourier para passar ao domínio da frequência. Porém, outros tipos de transformadas também são objeto de estudo no âmbito da análise modal operacional, tais como a transformada de Wavelet e de Hilbert, que não foram tratadas nesta revisão. As transformadas de Wavelet e Hilbert são transformadas efetivas para a análise de sinais não estacionarias devido a que fornecem informação das frequências com uma localização no tempo, em conjunto com a informação da energia e da frequência. $O$ uso desse tipo de transformadas também é adequado para a análise de sistemas não lineais (Uhl e Klepka, 2005) ,(Han, Li e Li, 2007).

No entanto, as diferentes técnicas ou métodos descritos nesta revisão para avaliar parâmetros modais podem ser combinados com o uso das transformadas Wavelet e de Hilbert, como são: estimação de funções de correlação, o método do decremento aleatório, a técnica SVD, a transformação linear do espectro (FSDD), e os métodos que usam transmissibilidade. Exemplos desses tipos de combinações são abordados em estudos como em Lardies, Ta et al. (2004), que combina o uso do método do decremento aleatório, com a análise da transformada de Wavelet. O primeiro método para avaliar a resposta livre do sistema é o segundo para passar ao domínio da frequência e extrair os parâmetros modais. Em Le e Paultre (2013) usam a transformada de Wavelet com dados de funções de correlação e fazem uma decomposição em valores singulares. Os valores singulares são utilizados para avaliar as frequências e os amortecimentos, e os vetores singulares para avaliar os modos de vibração, da mesma forma como foi descrita no método FDD, mas esses parâmetros são estimados ao longo do tempo, possibilidade que oferece a transformada de Wavelet. Em Yan e Ren (2013) é usado o conceito de transmissibilidade entres espectros de resposta para avaliar parâmetros modais, conceito descrito nesta revisão, mas em combinação com o uso da transformada de Wavelet para avaliar a resposta espectral.

Na aplicação da transformada de Wavelet uma das variáveis mais importantes para o correto uso dessa técnica refere-se à escolha correta da função Wavelet mãe que controla a resolução em tempo e frequência da análise. Umas das Wavelet mãe mais conhecidas e aplicadas é a função de Morlet e Morlet modificada, porém, essas funções contêm uma serie de parâmetros que devem ser adequadamente estabelecidos. Diferentes trabalhos como, Hoa, Tamura et al. (2010), Le e Tamura (2009) e Hamtaei e Anvar (2008) estudam aproximações diferentes dessas funções com intuito de melhorar a resolução em frequência e tempo, e dessa forma melhorar a determinação dos parâmetros modais, para o caso especifico de análise modal operacional. 


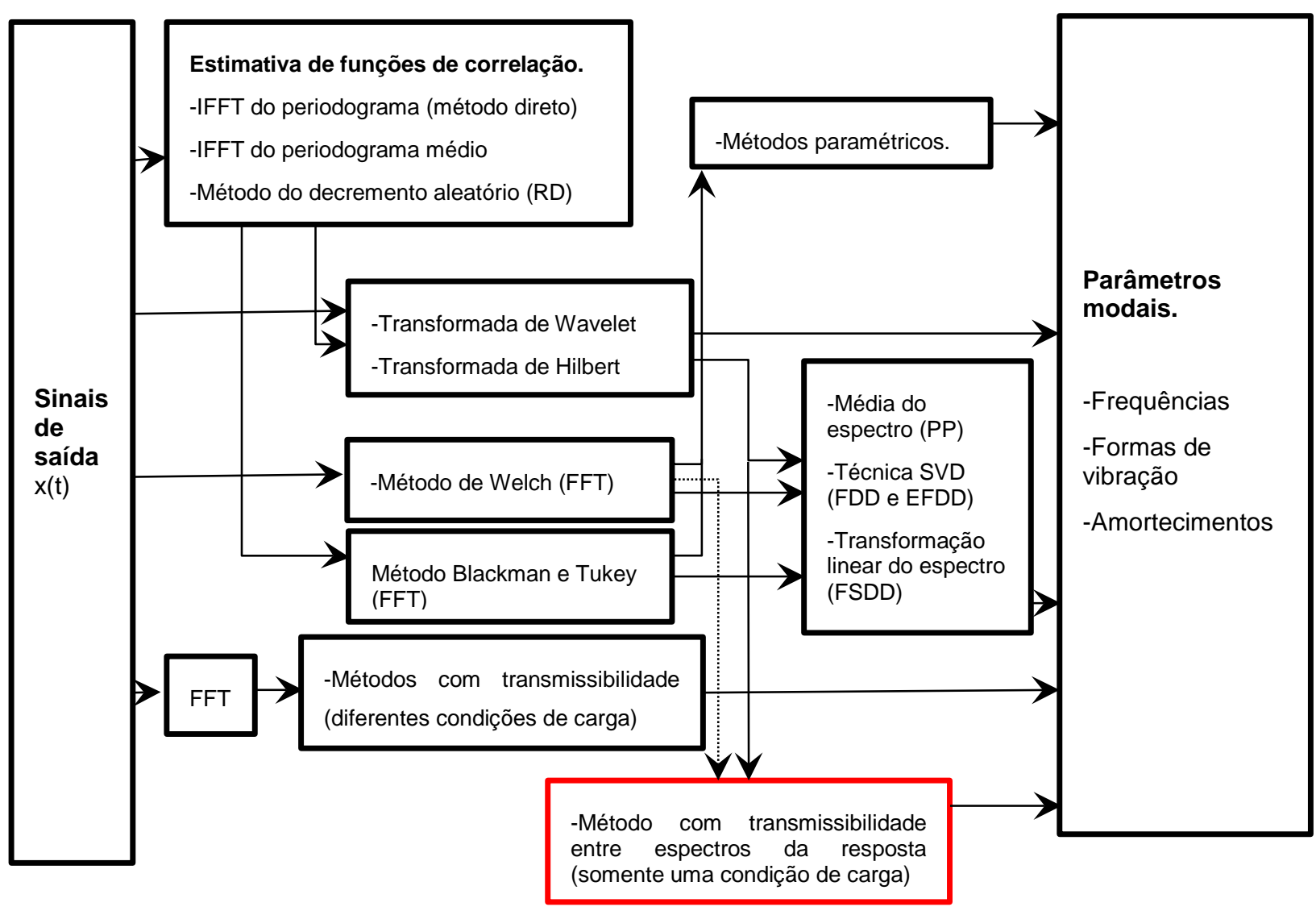

Figura 3-2 Sistematização dos principais métodos na análise modal operacional no domínio da frequência

Para o caso da transformada de Hilbert, ela é baseada na chamada técnica Empirical Mode Decomposition (EMD) (Huang et al., 1998). Nessa técnica estabelece-se uma decomposição do sinal em uma série de dados intrínsecos quase estacionários chamados de Intrinsic Mode Functions IMFs, através de um processo conhecido como shifting process. As frequências contidas em cada IMFs só dependem do sinal original e podem ser consideradas como uma classe de função de resposta modal. Dessa forma, aplicando o EMD, têm-se trabalhos como em Yu e Ren (2005) e Han, Li et al (2007), os quais adoptam o uso do EMD como ferramenta de pré-processamento do sinal para fazer uma decomposição da resposta e aplicar uma técnica de identificação modal. Também em He, Hua et al. (2011) foi combinado o método EMD com o método do decremento aleatório. Em que, primeiro foram avaliadas as funções IMFs para logo processar os dados pelo decremento aleatório e obter as funções de livre decaimento. As funções de livre decaimento são expressas analiticamente em termos de parâmetros modais e um problema de optimização é resolvido para obter os parâmetros modais de cada função. 
Finalmente, na Figura 3-3 apresenta-se um esquema em que se sistematizam os principais métodos de identificação para a análise modal operacional no domínio da frequência. 


\section{CAPÍTULO 4. MÉTODO BASEADO EM MATRIZES DE TRANSMISSIBILIDADE COM DENSIDADE ESPECTRAL VIA SVD (PSDTM-SVD).}

Recentes metodologias baseadas em conceitos de transmissibilidade têm sido surgidas como alternativas para a identificação de parâmetros modais de estruturas sob carregamentos em condições de operação. A identificação de parâmetros modais nesses tipos de metodologias é independente das características do carregamento aplicado na estrutura sendo isso uma importante vantagem com respeito às metodologias anteriores que supõem a excitação como ruído branco. A base dessas metodologias é o fato que as funções de transmissibilidade convergem a uma relação de amplitudes de modos de vibração que não dependem do espectro da excitação quando tendem em um pólo do sistema. Assim, estabelecendo uma relação inversa de uma subtração de duas funções de transmissibilidade dos mesmos graus de liberdade, chamada de função racional, podem-se obter os pólos do sistema, levando em consideração que somente um subconjunto dos pólos são os pólos do sistema. As primeiras aproximações consideram o uso de diferentes condições de carregamento para obter funções de transmissibilidade. No entanto, diferentes condições de carregamento seriam difíceis de obter em grandes estruturas. Uma solução alternativa devido a essa dificuldade é a partir do uso da transmissibilidade em base à densidade espectral de potência, que para diferentes condições de referência convergem na mesma relação de amplitudes dos modos de vibração, portanto, é possível usar da mesma maneira uma relação da inversa da subtração entre duas funções de transmissibilidade, para obter parâmetros modais com uma única condição de carga. Assim, da mesma forma, nessa subtração entre funções de transmissibilidade com densidade espectral, só um subconjunto de pólos são os pólos do sistema. De tal modo que em Yan e Ren (2012), um recurso usado para reduzir o risco de encontrar picos nas funções racionais que não são frequências naturais do sistema é a média normalizada das diferentes funções.

Neste capítulo é proposto o uso da técnica de valores singulares sobre matrizes de transmissibilidade de densidade espectral que convergem nas linhas à mesma relação de amplitudes dos modos vibração nos pólos do sistema. As matrizes de transmissibilidade propostas são linearmente dependentes nas colunas ou de posto 1 , quando convergem aos pólos do sistema, portanto, os valores singulares convergem para zero e podem ser usados para determinar frequências naturais. Assim, é proposto um produtório entre as inversas das médias dos valores singulares de cada matriz de transmissibilidade para a identificação das 
frequências naturais. Similarmente, o primeiro vetor singular à esquerda de cada matriz de transmissibilidade converge para as formas modais nos pólos do sistema. Desse modo, é proposta uma média dos diferentes primeiros vetores singulares à esquerda de cada matriz de transmissibilidade para estimar os modos de vibração. O método proposto foi verificado através de uma análise numérica, na qual uma viga foi excitada com ruído colorido contendo uma frequência predominante. Duas diferentes configurações de localização do carregamento foram analisadas. Igualmente, dados reais de vibração de uma ponte simplesmente apoiada foram usados. Os resultados de ambas as análises, numérica e dados de vibração real demostraram a capacidade do método proposto para a identificação de parâmetros modais. Os resultados foram comparados com os obtidos pelos métodos PSDT e FDD detalhados no Capítulo 3.

\subsection{IDENTIFICAÇÃO DE PARÂMETROS MODAIS USANDO PSDTM-SVD}

No Capítulo 3 é mostrado como no método baseado em transmissibilidade com densidade espectral, funções de transmissibilidade entre dois pontos $\boldsymbol{o}$ e $\boldsymbol{j}$ com referência de saída diferente $\boldsymbol{k}$ e $\boldsymbol{z}$ convergem à mesma relação de amplitudes de modos de vibração nos pólos do sistema, $i \omega=i \omega_{l}$, assim.

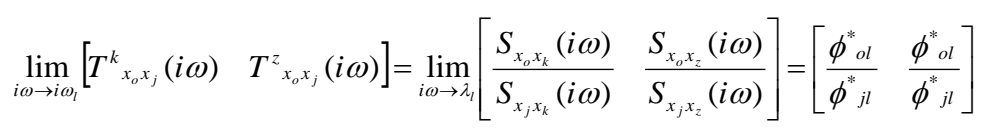

Da mesma forma, é possível definir uma linha de funções de transmissibilidade com referências de saída diferentes, $\mathbf{z}_{\mathbf{i}}$, como.

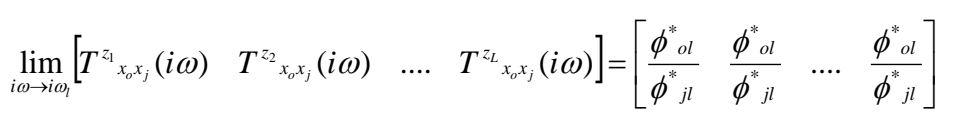

Em que, L é o número total de pontos de medição. Igualmente, pode-se fazer uma variação para diferentes pontos, $\boldsymbol{o}_{i}$, e construir uma matriz de transmissibilidade, da seguinte forma.

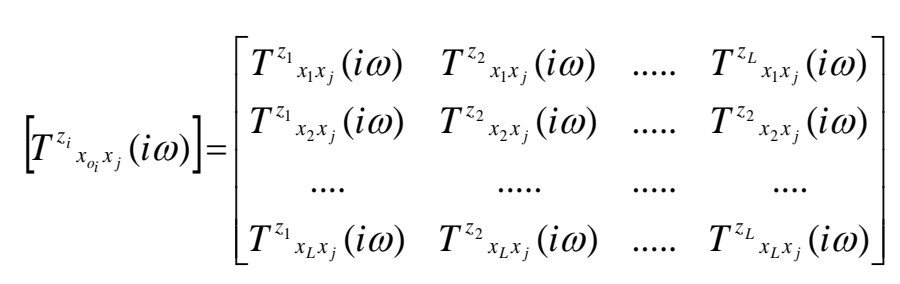

Diferentes matrizes quadradas de transmissibilidade $L x L$ surgem a partir de realizar uma variação do ponto j, como. 


$$
\left.\left.\left\{\left[T^{Z_{i}}{ }_{x_{0 i} X_{1}}(i \omega)\right] \mid T^{Z_{i}}{ }_{x_{o_{i}} x_{2}}(i \omega)\right] \ldots \mid T^{Z_{i}}{ }_{x_{0 i} x_{L}}(i \omega)\right]\right\}
$$

As matrizes estabelecidas convergem à seguinte relação quando se aproximam nos pólos do sistema.

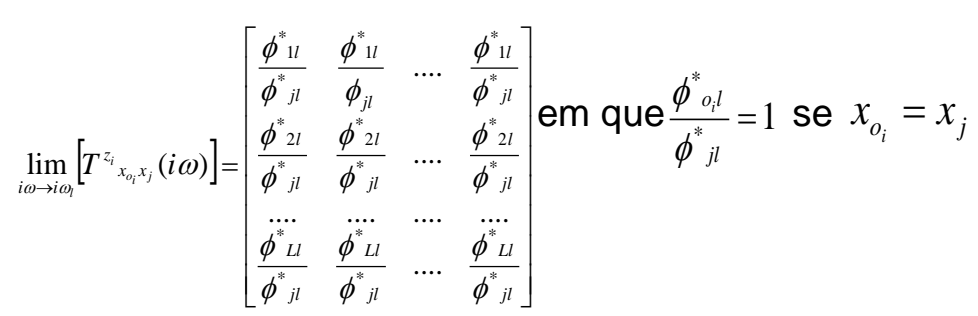

Pode-se notar na Equação (4-5) que as matrizes obtidas são linearmente dependentes nas colunas quando convergem aos pólos do sistema. Essa característica matricial de dependência linear entre as colunas pode ser analisada a través do conceito do posto de uma matriz que se define como o número de linhas não nulas quando a mesma é escrita na forma reduzida escalonada por linhas ou equivalente ao número de colunas independentes da matriz. Uma forma direta de se avaliar o posto de uma matriz é através da avaliação dos valores próprios da matriz. O número de valores próprios diferentes de zero é equivalente ao posto da matriz. Igualmente, poder-se-ia avaliar a partir dos valores singulares que têm uma direta relação com os valores próprios. Isso significa que o posto da matriz de transmissibilidade proposta (Equação (4-3)), quando se aproxima aos pólos do sistema $\lambda_{1}$, será igual a 1 , o que implica também que a partir do segundo valor singular convergirão para zero para, $i \omega=i \omega_{l}$. Então, a partir do uso da inversa dos valores singulares podem-se avaliar as frequências naturais do sistema.

Dessa forma, define-se uma decomposição em valores singulares das matrizes de transmissibilidade $\left[T^{z_{i}}{ }_{x_{0_{i}} x_{j}}(i \omega)\right]$, como.

$$
\left[T^{z_{i}}{ }_{x_{o_{i}} x_{j}}(i \omega)\right]=\left[U^{j}(i \omega)\right]_{L \times L}\left[\Sigma^{j}(i \omega)\right]_{L \times L}\left[V^{j}(i \omega)\right]_{L \times L}{ }^{H}
$$

Em que $\left[U^{j}(i \omega)\right]$ e $\left[V^{j}(i \omega)\right]$ são os vetores singulares à esquerda e à direita da matriz $\left[T^{z_{i}}{ }_{x_{o_{i}} x_{j}}(i \omega)\right]$, respectivamente, $\left[\Sigma^{j}(i \omega)\right]$ é uma matriz diagonal contendo os valores singulares da matriz $\left[T^{z_{i}}{ }_{\alpha_{o_{i}} x_{j}}(i \omega)\right]$, com $\sigma_{1}(i \omega) \geq \sigma_{2}(i \omega) \geq \ldots \geq \sigma_{L}(i \omega)$ e $\boldsymbol{H}$ é a transposta conjugada. Para cada matriz de transmissibilidade $\left[T^{z_{i}}{ }_{\alpha_{i} x_{j}}(i \omega)\right]$ são avaliados os valores singulares, como $\Sigma(i \omega)^{j}=\left[\begin{array}{llll}\sigma(i \omega)^{j}{ }_{1} & \sigma(i \omega)^{j}{ }_{2} & \ldots & \sigma(i \omega)^{j}{ }_{L}\end{array}\right]$. 
Então, no método PSDTM-SVD é proposto um cálculo entre valores singulares para avaliar as frequências naturais em dois passos: 1) calcular a média aritmética da inversa dos valores singulares, a partir do segundo valor singular em cada matriz de transmissibilidade, como.

$$
\begin{aligned}
\hat{\Sigma}(i \omega)^{-1}= & \frac{1}{L} \sum_{j=1}^{L} \frac{1}{\Sigma(i \omega)^{j}} \\
& =\frac{1}{L} \sum_{j=1}^{L}\left[\begin{array}{llll}
\frac{1}{\sigma(i \omega)^{j}{ }_{2}} & \frac{1}{\sigma(i \omega)^{j}{ }_{3}} & \cdots & \frac{1}{\sigma(i \omega)^{j} L}
\end{array}\right]=\left[\begin{array}{llll}
\frac{1}{\hat{\sigma}(i \omega)_{2}} & \frac{1}{\hat{\sigma}(i \omega)_{3}} & \cdots & \frac{1}{\hat{\sigma}(i \omega)_{L}}
\end{array}\right]
\end{aligned}
$$

2) calcular um produtório entre as médias dos valores singulares de cada matriz de transmissibilidade.

$$
\pi(i \omega)=\prod_{i=2}^{L} \frac{1}{\hat{\sigma}(i \omega)_{i}}
$$

A função $\pi(i \omega)$ contém picos de ressonância que são as frequências naturais do sistema. Logo, na seção dos cálculos deste capítulo será discutida uma razão para escolher um produtório entre as médias das inversas dos valores singulares. Uma vez que as frequências de ressonância do sistema são conhecidas, é possível derivar as formas modais a partir do uso do primeiro vetor singular $\left[U^{j}\left(i \omega_{l}\right)\right]_{1}$ na frequência de ressonância, i $\omega_{l}$ para cada matriz de transmissibilidade $\left[T^{z_{i}} x_{o_{i} x_{j}}(i \omega)\right]$, assim uma média dos diferentes vetores singulares faz uma estimativa da forma modal, como.

$$
\hat{U}\left(i \omega_{l}\right)_{1}=\frac{1}{L} \sum_{j=1}^{L} U^{j}\left(i \omega_{l}\right)_{1}
$$

Os vetores singulares $[U(i \omega)]$ são produto de avaliar o problema dos vetores próprios da matriz $\left.\left[T^{z_{i}}{ }_{x_{o_{i}} x_{j}}(i \omega)\right] T^{Z_{i}}{ }_{o_{o_{i}} x_{j}}(i \omega)\right]^{T}$, assim, a partir desse conceito, é possível demostrar que o primeiro vetor singular $[U(i \omega)]_{1}$, converge às formas modais do sistema quando a matriz $\left[T^{z_{i}}{ }_{o_{i} x_{j}}(i \omega)\right]\left[T^{z_{i}}{x_{o_{i}} x_{j}}_{(i \omega)}\right]^{T}$ tende aos pólos do sistema. Dessa forma, pode-se resolver um problema de valores e vetores próprios da matriz $\left.\left[T^{Z_{i}}{ }_{{ }_{o_{i}} x_{j}}(i \omega)\right] T^{Z_{i}}{ }_{X_{o_{i}} x_{j}}(i \omega)\right]^{T}$ para $i \omega=i \omega_{l}$, como. 


$$
\begin{aligned}
& \left.\left.\lim _{i \omega \rightarrow i \omega_{l}} \mid\left[T^{z_{i}}{ }_{o_{o_{i}} x_{j}}(i \omega)\right]\left[T^{z_{i}}{ }_{o_{\sigma_{i}} x_{j}}(i \omega)\right]^{T}-\lambda_{i}(i \omega) \cdot I\right]\right\}(U(i \omega)\}_{i}=0
\end{aligned}
$$

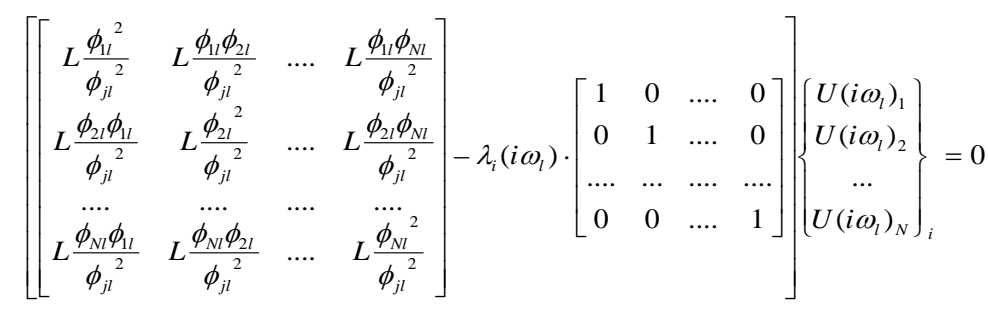

Os valores próprios da matriz $\left.\left[T^{z_{i}}{ }_{x_{o_{i}} x_{j}}(i \omega)\right] T^{z_{i}}{ }_{x_{o_{i}} x_{j}}(i \omega)\right]^{T}$ para $i \omega=i \omega_{l} \quad$ ficam determinados por.

$$
\lambda\left(i \omega_{l}\right)=\left[\begin{array}{llll}
L \frac{\phi_{1 l}{ }^{2}+\phi_{2 l}{ }^{2}+\ldots+\phi_{L l}{ }^{2}}{\phi_{j l}{ }^{2}} & 0 & \ldots & 0
\end{array}\right]
$$

Assim, o primeiro vetor singular pode ser avaliado da matriz $\lim _{i \omega \rightarrow \lambda_{l}}\left[T^{Z_{i}}{ }_{x_{o_{i}} x_{j}}(i \omega)\right]\left[T^{z_{i}}{ }_{\alpha_{0_{i}} x_{j}}(i \omega)\right]^{T}$ e é determinado por.

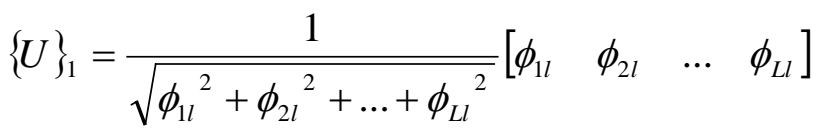

O anterior revela que o primeiro vetor singular de $[U(i \omega)]$ das diferentes matrizes de transmissibilidade propostas para valores próximos na ressonância é proporcional ao modo de vibração do sistema. Cabe salientar, que não acontece o mesmo com o primeiro vetor singular $\left[V^{j}(i \omega)\right]_{1}$, o qual se avalia a partir do problema de valores e vetores próprios da matriz $\left[T^{Z_{i}}{ }_{o_{o_{i}} x_{j}}(i \omega)\right]^{T}\left[T^{Z_{i_{i}}}{ }_{\alpha_{o_{i}} x_{j}}(i \omega)\right]$. O primeiro vetor $\left[V^{j}(i \omega)\right]_{1}$ converge na ressonância a uma relação, como.

$$
\{V\}_{1}=\frac{1}{L}\left[\begin{array}{llll}
\sqrt{L} & \sqrt{L} & \ldots & \sqrt{L}
\end{array}\right]
$$

Finalmente, uma fácil implementação computacional do método proposto pode ser gerada a partir de seguir o diagrama de fluxo mostrado na Figura 4-1. 


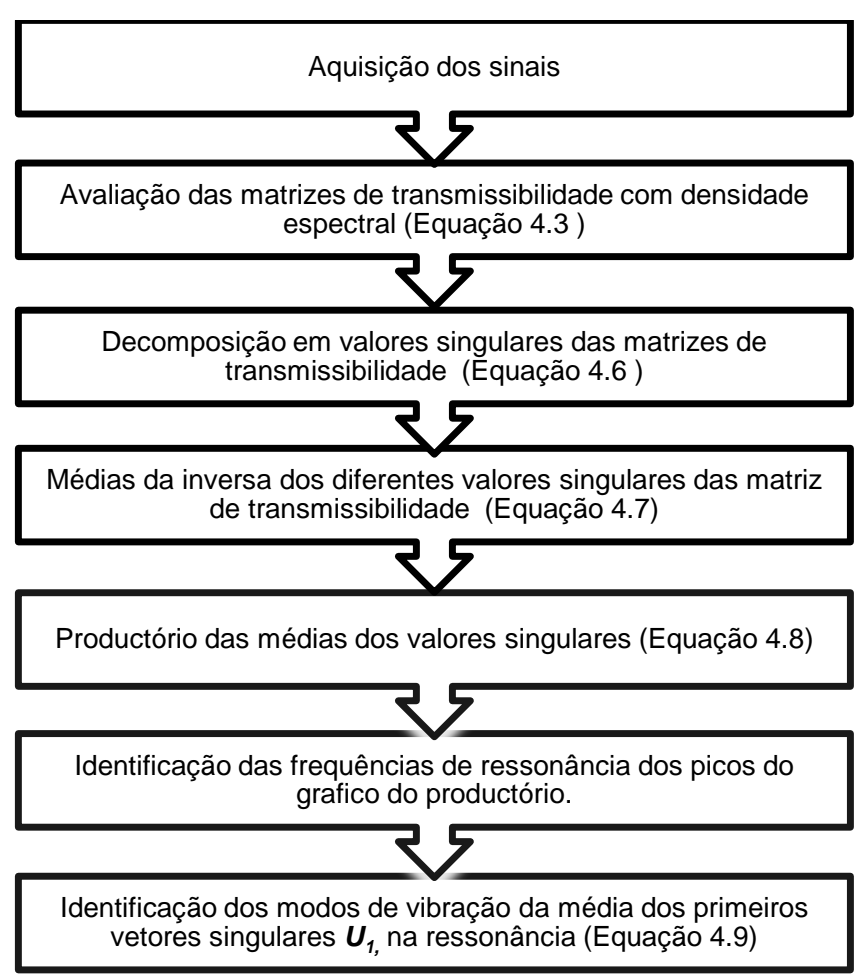

Figura 4-1 Diagrama de fluxo do método proposto PSDTM-SVD.

\subsection{EXEMPLO NUMÉRICO}

Com o fim de mostrar o desempenho do método proposto será processado uma simulação de dados sobre um sistema linear invariante no tempo. O sistema será o mesmo estudado no Apêndice $A$, contendo as mesmas características geométricas, de rigidez, massa e amortecimento (ver Figura A-3). Nesse exemplo foram analisadas duas configurações de carga diferentes com forças não correlacionadas de um tempo total de 300 seg e que apresentam um espectro de ruído colorido com uma frequência de excitação predominante igual a $12 \mathrm{~Hz}$. O ruído colorido foi gerado a partir de filtrar um ruído branco por um sistema de um só grau de liberdade e selecionar as velocidades da resposta. O sistema SDF contém uma frequência de $12 \mathrm{~Hz}$ com um amortecimento de $5 \%$. A frequência de amostragem da excitação foi definida de $350 \mathrm{~Hz}$. As duas configurações de carga foram dadas por: Carga 1, com um carregamento localizado nos graus de liberdade 5 e 14 e, Carga 2, com um carregamento localizado nos graus de liberdade 5,8,11,14 e 17 . Um exemplo das características da excitação em termos de função de correlação e a densidade espectral são mostrados na Figura 4-2. 

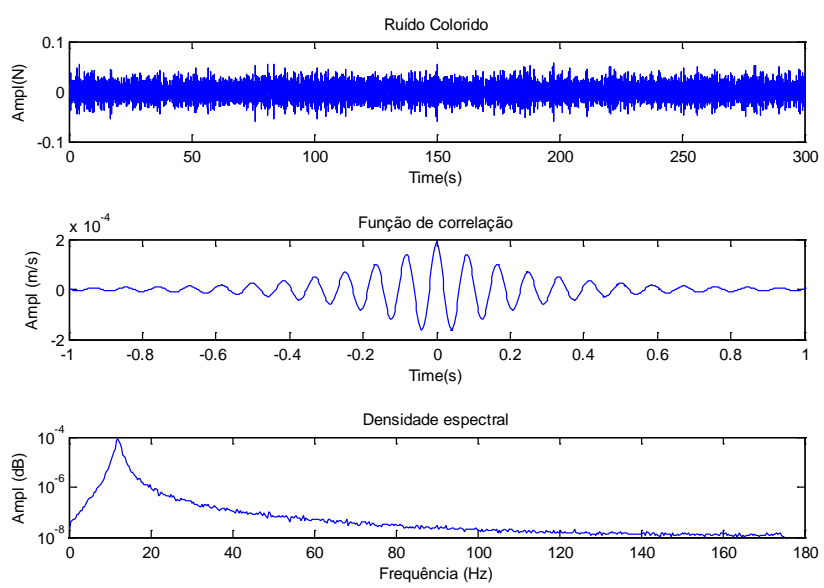

Figura 4-2 Características da excitação do exemplo numérico.

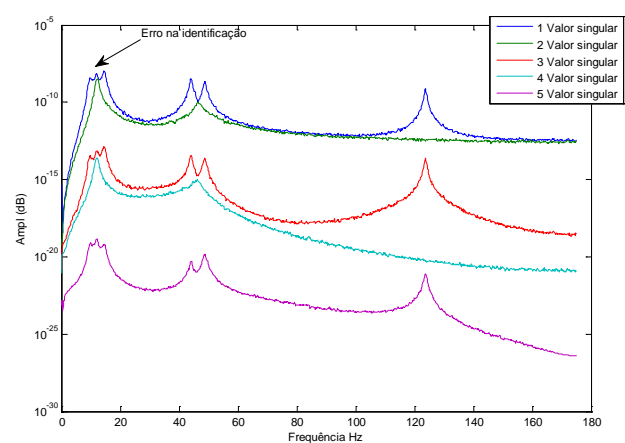

a)

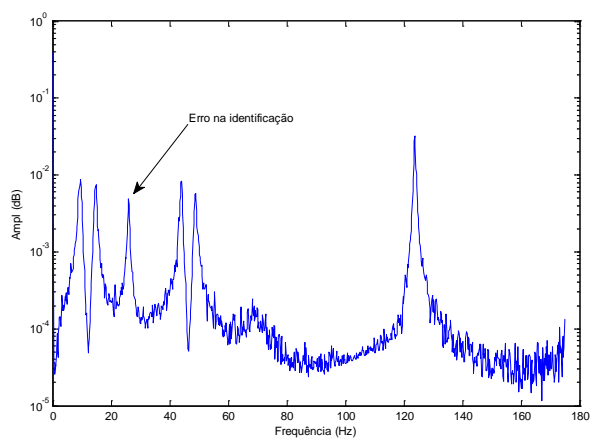

b)

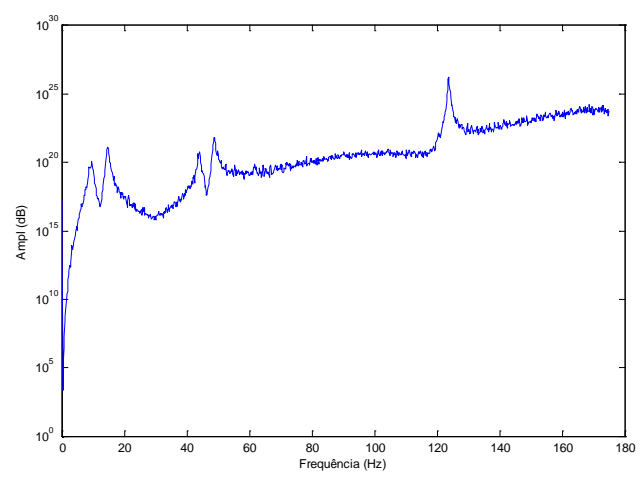

c)

Figura 4-3 Resultados dos métodos a) FDD, b) PSDT, c) PSDTMSVD para a Carga 1.

Com o objetivo de analisar a robustez da metodologia proposta, PSDTM-SVD, foi realizada uma comparação com os resultados dos métodos FDD e PSDT frente aos dois tipos de carregamentos indicados. Para as diferentes metodologias em análise foram 
avaliadas as funções de densidade espectral pelo método de Welch, no qual foi usada uma janela tipo Hanning com um tamanho de $2^{11}$ dados, uma sobreposição do $50 \%$ e um tamanho da transformada de Fourier de $2^{11}$. Dessa forma, são mostrados nas Figura 4-3 e Figura 4-4 os resultados para as diferentes metodologias, FDD, PSDT e PSDTM-SVD. As frequências naturais do sistema nas diferentes metodologias são obtidas dos picos dos gráficos de: os primeiros valores singulares da decomposição da matriz de densidade espectral para o método FDD, média normalizada das inversas das funções racionais para o método PSDT e o produtório da média da inversa dos valores singulares das matrizes de transmissibilidade para o método proposto, PSDTM-SVD (Equação (5-8)).

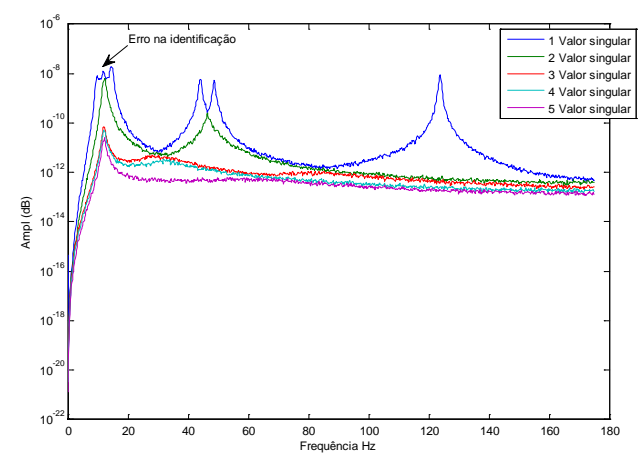

a)

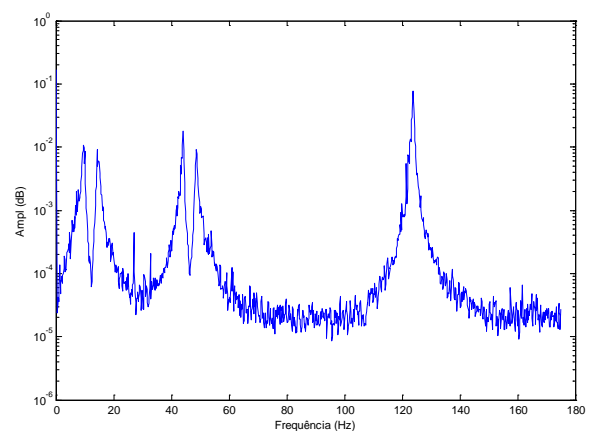

b)

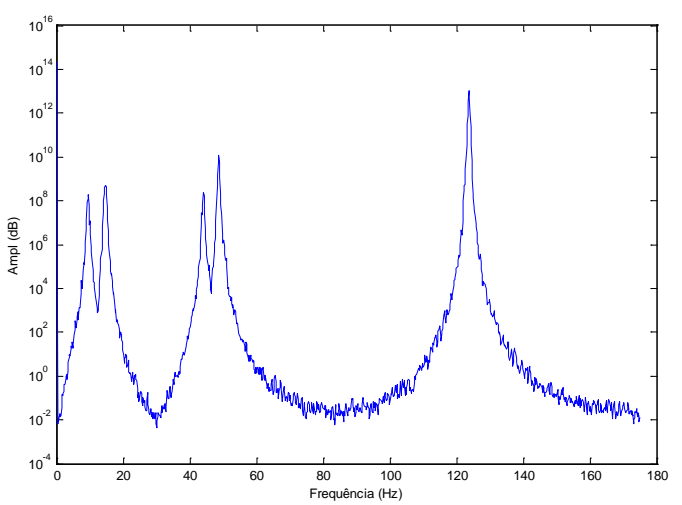

c)

Figura 4-4 Resultados dos métodos a) FDD, b) PSDT, c)PSDTMSVD para a Carga 2.

As frequências naturais identificadas por cada método têm sido comparadas com o valor teórico do modelo estrutural como é mostrado na Tabela 4-1. Os resultados mostraram que para o modelo estrutural proposto e os tipos de carregamentos indicados, o primeiro método (FDD), para os dois casos de carga, existiu uma introdução no espectro da resposta de uma frequência de ressonância que não faz parte do sistema devido à excitação de ruído colorido com uma frequência predominante utilizada. Para o método PSDT no caso de carregamento, Carga 1, encontrou-se uma existência de um pico de ressonância não pertencente ao modelo estrutural, igual $25.98 \mathrm{~Hz}$. A existência dessa ressonância errônea se 
deve principalmente ao uso da inversa da função racional que algumas vezes podem conter pólos que não são do sistema (Devriendt e Guillaume, 2007). Assim, neste caso específico, o método PSDT a partir de utilizar uma média de diversas inversas da função racional, não consegue eliminar completamente os picos errados. Particularmente, neste exemplo numérico quando o modelo só contém um, dois, ou três graus de liberdade carregados, foi encontrado que o método PSDT tem falhas na identificação devido à introdução de frequências adicionais que não são parte do sistema. Porém, esse método é capaz de identificar adequadamente as frequências naturais, sem a introdução de picos adicionais, quando todos os graus de liberdade do modelo são carregados.

Tabela 4-1 Frequências naturais identificadas para o exemplo

\begin{tabular}{|c|c|c|c|c|c|c|c|}
\hline \multirow[b]{2}{*}{ Modo } & \multirow{2}{*}{$\begin{array}{c}\text { Valor } \\
\text { Teórico } \\
(\mathrm{Hz})\end{array}$} & \multicolumn{3}{|c|}{ Estimado $(\mathrm{Hz})$} & \multicolumn{3}{|c|}{ Erro(\%) } \\
\hline & & FDD & PSDT & $\begin{array}{c}\text { PSDTM- } \\
\text { SVD }\end{array}$ & FDD & PSDT & $\begin{array}{c}\text { PSDTM- } \\
\text { SVD }\end{array}$ \\
\hline \multicolumn{8}{|c|}{ Carga 1} \\
\hline 1 & 9.539 & $\begin{array}{c}9.91 \\
11.96\end{array}$ & 9.57 & 9.57 & $\begin{array}{c}3.89 \\
*\end{array}$ & 0.33 & 0.33 \\
\hline 2 & 14.616 & 14.53 & $\begin{array}{l}14.53 \\
25.98\end{array}$ & 14.7 & 0.59 & $\begin{array}{c}0.59 \\
*\end{array}$ & 0.58 \\
\hline 3 & 44.049 & 44.09 & 43.92 & 43.75 & 0.09 & 0.29 & 0.68 \\
\hline 4 & 48.700 & 48.88 & 48.88 & 48.88 & 0.37 & 0.37 & 0.37 \\
\hline 5 & 123.486 & 123.7 & 123.7 & 123.7 & 0.17 & 0.17 & 0.17 \\
\hline \multicolumn{8}{|c|}{ Carga 2} \\
\hline 1 & 9.539 & $\begin{array}{c}9.74 \\
11.79\end{array}$ & 9.22 & 9.57 & $\begin{array}{c}2.11 \\
*\end{array}$ & 3.34 & 0.33 \\
\hline 2 & 14.616 & 14.53 & 15.04 & 14.7 & 0.59 & 2.90 & 0.58 \\
\hline 3 & 44.049 & 44.09 & 43.92 & 44.09 & 0.09 & 0.29 & 0.09 \\
\hline 4 & 48.700 & 48.71 & 49.22 & 48.71 & 0.02 & 1.07 & 0.02 \\
\hline 5 & 123.486 & 123.6 & 123.7 & 123.7 & 0.09 & 0.17 & 0.17 \\
\hline
\end{tabular}

*Identificação errada do método

No caso do método proposto, PSDTM-SVD, para os dois casos de carga, foram identificadas as frequências naturais do sistema sem a introdução de frequências adicionais. Dessa maneira, o método PSDTM-SVD neste exemplo numérico mostra a habilidade de reduzir ainda mais o risco de encontrar frequências adicionais para diferentes posições do carregamento e quantidade de graus de liberdade carregados. Para o método PSDTM-SVD é mostrado na Figura 4-5 um exemplo da decomposição em valores singulares só da matriz de transmissibilidade $\left[T^{z_{i}}{ }_{x_{o_{i}} x_{1}}(i \omega)\right]$ para os dois casos de carga, na que se pode observar como os valores singulares convergem para zero nos pólos do sistema. Assim, a 


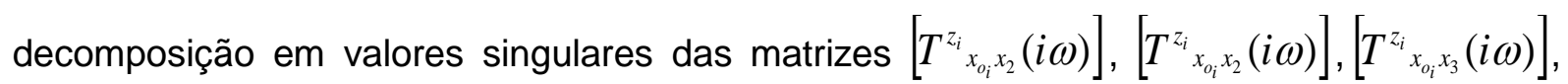
$\left[T^{z_{i}}{ }_{x_{\theta_{i}} x_{5}}(i \omega)\right]$ mostraram um comportamento similar. Pode-se observar que no primeiro caso de carga, quando só os graus de liberdade 5 e 14 são carregados, existe uma maior separação entre os valores singulares, comparado com a decomposição em valores singulares do segundo caso de carga. Também, observa-se que no caso de Carga 1 não todos os valores singulares converge para zero nos pólos do sistema. As anteriores observações são a causa da proposta de um produtório entre as inversas da média dos valores singulares de cada matriz de transmissibilidade como a estimativa das frequências naturais. Uma estimativa, por exemplo, através de uma média normalizada das médias dos valores singulares das matrizes de transmissibilidade foi descartada, devido a que, para o caso de Carga 1, em que, os valores singulares se encontram bem separados e não todos convergem para zero, geraria uma cancelamento das frequências naturais.

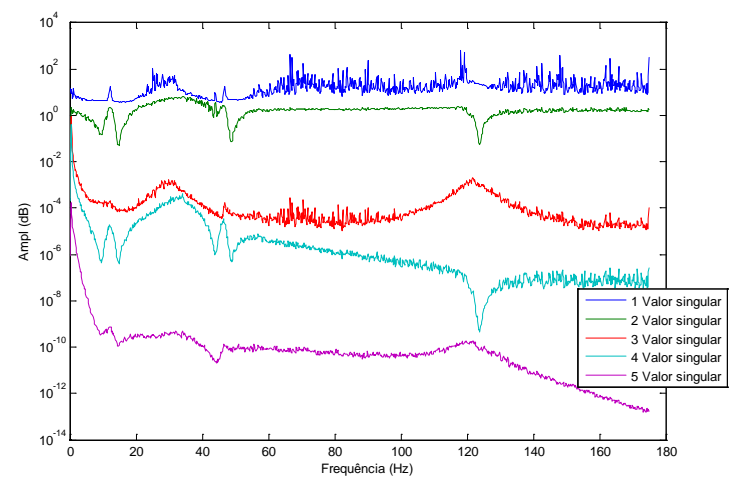

a) Caso de Carga 1

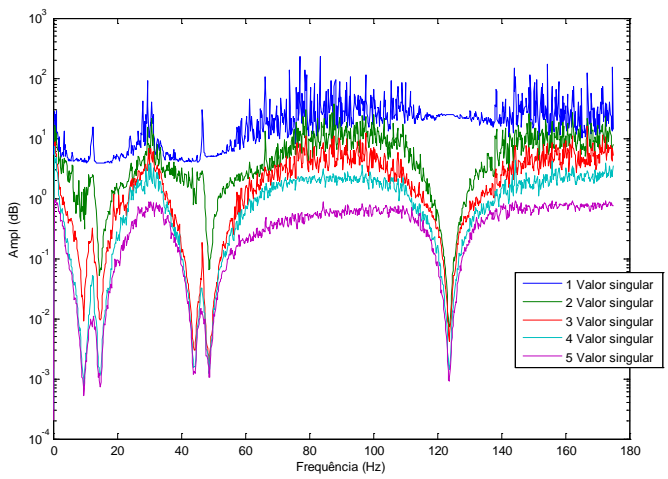

b) Caso de Carga 2

Figura 4-5 Decomposição em valores singulares da matriz de transmissibilidade $\left[T^{z_{i}}{ }_{o_{O_{i}} x_{1}}(i \omega)\right]$.

Uma vez que as frequências de ressonância são identificadas pelo método PSDTMVSD podem-se avaliar os modos de vibração através de uma média dos primeiros vetores singulares à esquerda das matrizes de transmissibilidade escolhidos na ressonância como é mostrado na Tabela 4-2. Uma comparação entre os modos identificados e teóricos é realizada através do indicador de correlação modal MAC, encontrando um adequado acordo entre eles, como é mostrado na Tabela 4-3. 
Tabela 4-2 Modos identificados com o método PSDTM-SVD

\begin{tabular}{ccccccccccc}
\hline \multirow{2}{*}{ GL } & \multicolumn{2}{c}{ Mode 1 } & \multicolumn{2}{c}{ Mode 2 } & \multicolumn{2}{c}{ Mode 3 } & \multicolumn{2}{c}{ Mode 4 } & \multicolumn{2}{c}{ Mode 5 } \\
\cline { 2 - 11 } & Teórico & Estimado & Teórico & Estimado & Teórico & Estimado & Teórico & Estimado & Teórico & Estimado \\
\hline 5 & 0.0315 & -0.3650 & -0.0361 & -0.5736 & -0.0376 & -0.4878 & -0.0325 & -0.4488 & 0.0068 & -0.0897 \\
8 & 0.0315 & -0.3824 & -0.0261 & -0.4120 & 0.0376 & 0.4904 & 0.0384 & 0.5285 & -0.0201 & 0.2657 \\
11 & 0.0000 & 0.0448 & -0.0019 & -0.0308 & 0.0000 & 0.0102 & 0.0120 & 0.1651 & 0.0695 & -0.9179 \\
14 & -0.0315 & 0.5129 & -0.0261 & -0.4137 & -0.0376 & -0.4370 & 0.0384 & 0.5352 & -0.0201 & 0.2661 \\
17 & -0.0316 & 0.5362 & -0.0361 & -0.5735 & 0.0376 & 0.4393 & -0.0325 & -0.4532 & 0.0068 & -0.0896 \\
\hline
\end{tabular}

Tabela 4-3 Comparação dos modos teóricos e estimados com o método PSDTMSVD

\begin{tabular}{crrrrr}
\hline Teórico/ & \multicolumn{5}{c}{ MAC } \\
Estimado & Modo 1 & Modo 2 & Modo 3 & Modo 4 & Modo 5 \\
\hline Modo 1 & 0.970 & 0.028 & 0.000 & 0.000 & 0.001 \\
Modo 2 & 0.028 & 1.000 & 0.000 & 0.005 & 0.008 \\
Modo 3 & 0.000 & 0.000 & 0.997 & 0.003 & 0.000 \\
Modo 4 & 0.000 & 0.005 & 0.003 & 1.000 & 0.045 \\
Modo 5 & 0.001 & 0.008 & 0.000 & 0.045 & 1.000 \\
\hline
\end{tabular}

\subsection{DADOS REAIS DE VIBRAÇÃO}

Nesta seção foram usados dados de um ensaio de vibração de uma ponte em condições de operação, como um estudo de caso para demostrar a habilidade do método proposto para a identificação de parâmetros modais. Os dados são extraídos de um estudo realizado anteriormente pelo autor (Araujo, Maldonado e Cho, 2011). Para verificar o método proposto foram identificadas as frequências naturais e formas modais da ponte usando o método PSDTM-SVD e foram comparados com os métodos FDD e PSDT. Nesta aplicação sobre os dados reais, o método PSDTM-SVD demostrou a capacidade para identificar parâmetros modais da estrutura. 


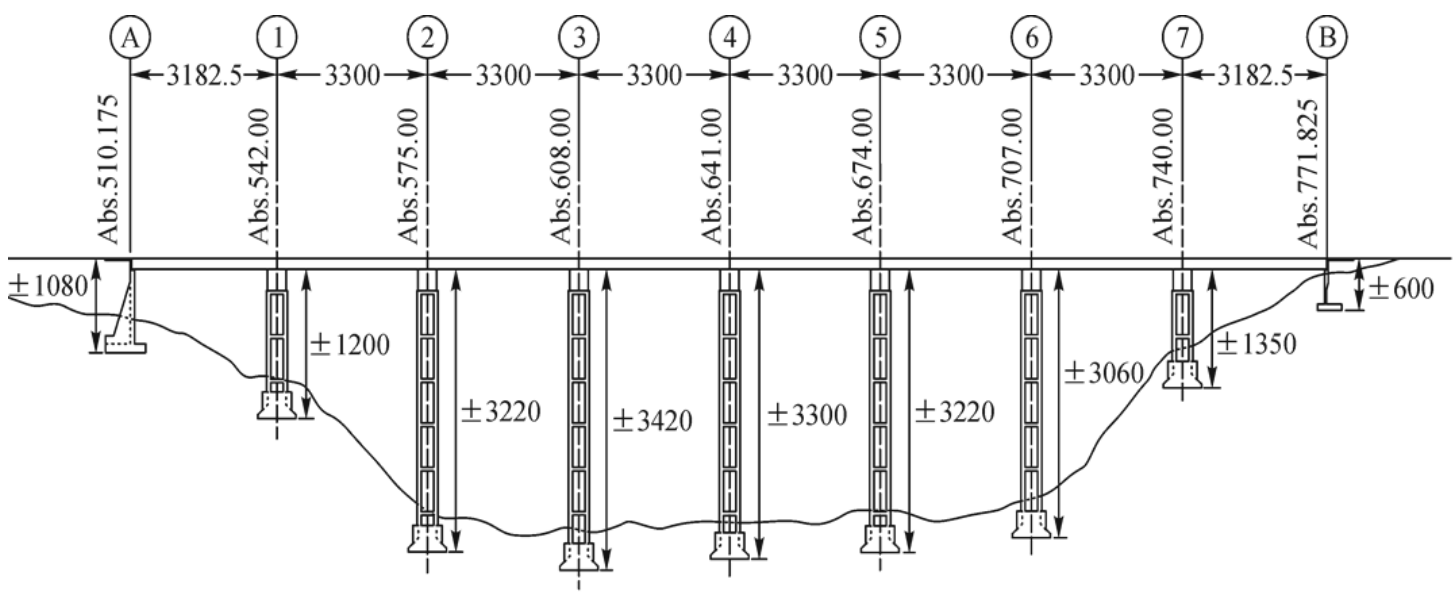

a)

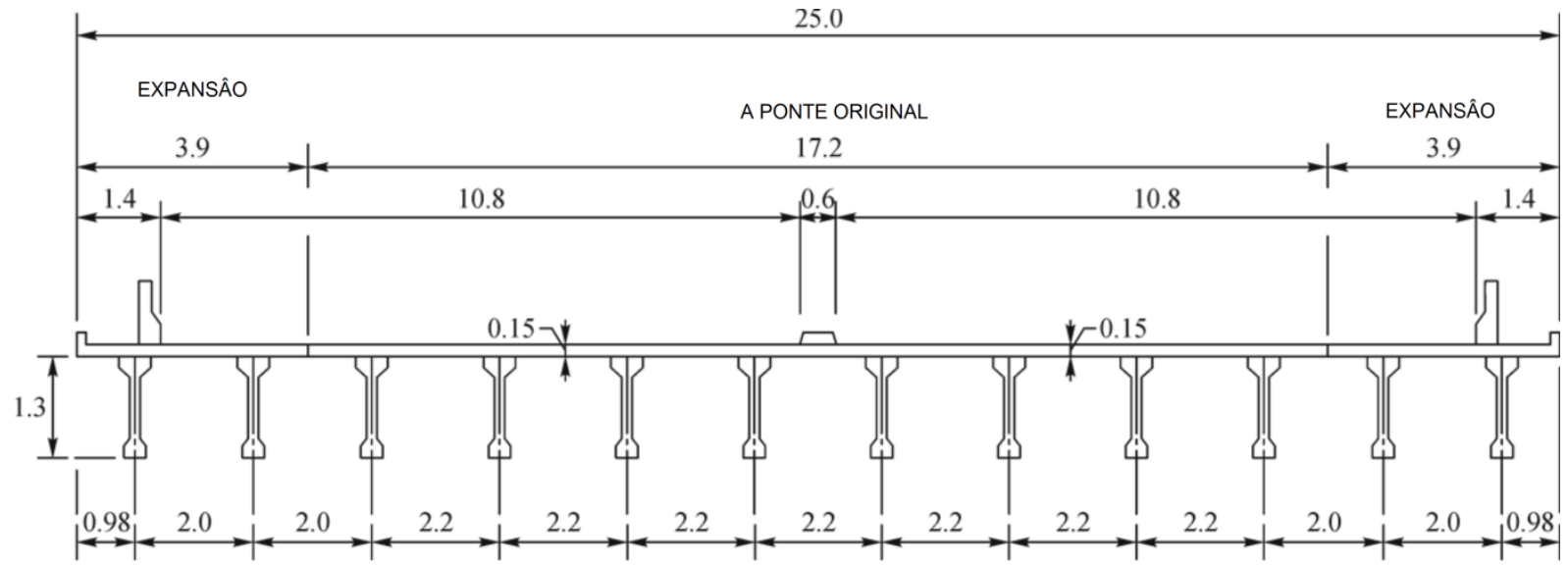

b.)

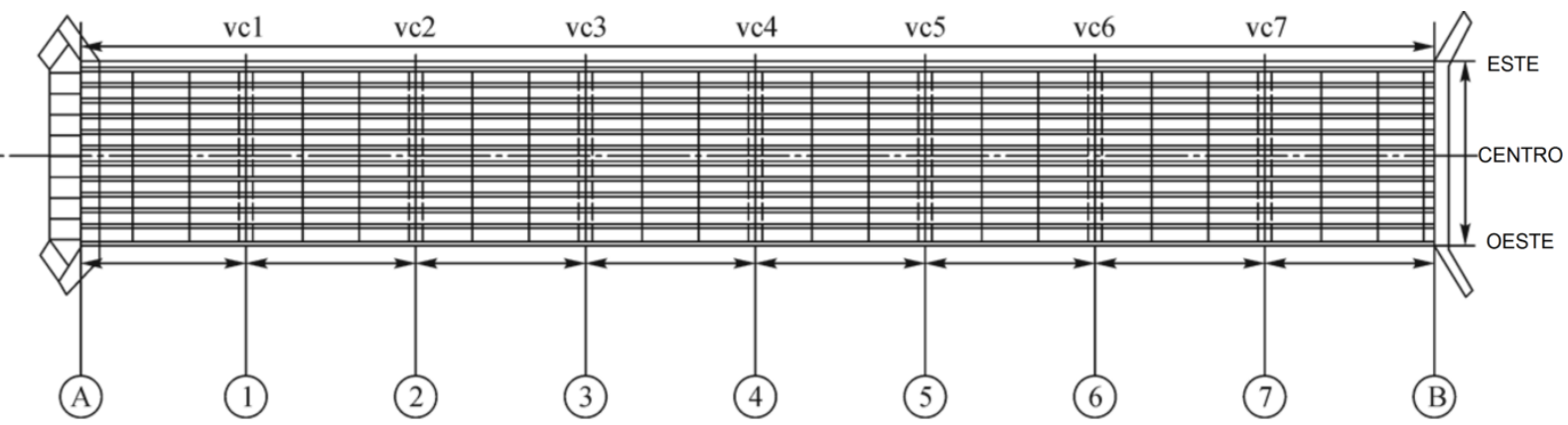

c.)

Figura 4-6 a) O corte longitudinal, b) o corte transversal da superestrutura e c) o detalhe da planta da ponte Garcia Cadena. 


\subsubsection{Descrição da ponte.}

A ponte Garcia Cadena é uma estrutura de concreto reforçado que foi construída em 1971 e é localizada na cidade de Bucaramanga, Colômbia. A ponte é caracterizada por uma superestructura que consiste em um sistema de vigas pós-tracionadas simplesmente apoiadas. Em 1993, a ponte foi expandida pela adição de duas vigas em cada lado. Atualmente, a ponte tem um comprimento de 261,65m e uma largura de $25 \mathrm{~m}$ (estrada de mão dupla com três faixas), um total de oito vãos e sete pilares. Na Figura 4-7 é mostrada uma fotografia da ponte, e na Figura 4-6 é mostrado o corte longitudinal, o corte transversal da superestrutura e o detalhe da planta.

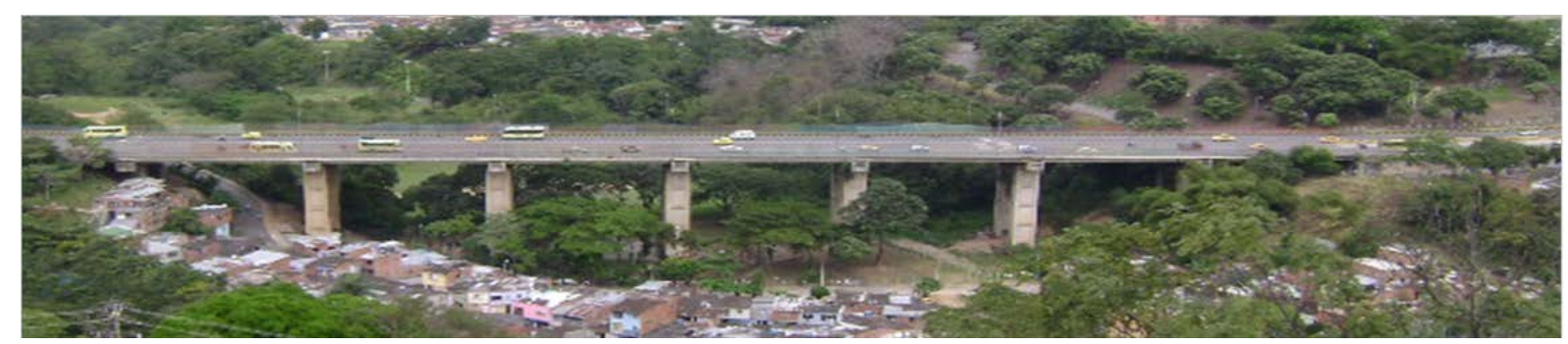

Figura 4-7 Fotografia da ponte Garcia Cadena.

\subsubsection{Ensaio de vibrações}

O ensaio de vibrações foi realizado no ano de 2010, no qual foram usados dois acelerômetros tri-axiais de força balanceada (FB, ES-T Kinemetrics) e um sistema de aquisição de dados, K2 (AltusDigital Recorder, Kinemetrics Company). O sistema K2 tem nove canais de aquisição e outros três canais que são ocupados por um acelerômetro triaxial interno. Com esse equipamento foram registradas acelerações verticais em diferentes pontos dos vãos da estrutura, ao longo dos eixos Este, Oeste e Central. O ensaio consistiu na definição de sete pontos internos em todos os vãos, localizando o acelerômetro interno do sistema de aquisição $\mathrm{K} 2$, chamado de acelerômetro de referência, em um ponto específico de medição. Os outros acelerômetros foram mobilizados nos outros pontos de medição. Assim, foi realizado um total de três configurações de localização dos acelerômetros (setups), como mostrado na Figura 4-8. Desta maneira, foram registradas para cada configuração entre 5 a 6 min de vibrações como uma frequência de amostragem de $200 \mathrm{~Hz}$. 


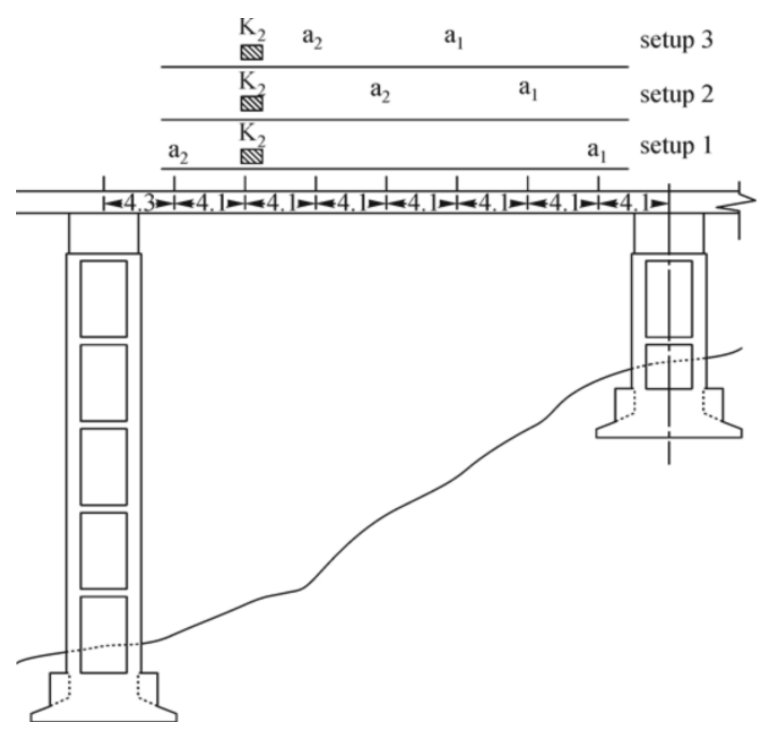

Figura 4-8 Configurações dos acelerômetros.

\subsubsection{Identificação de parâmetros modais}

Neste estudo de caso foram realizadas medições de vibrações para todos os vãos da estrutura nos eixos Este, Oeste e Central. No entanto, devido à quantidade de informação que pode surgir para todos os vãos, nesta análise só os resultados para um dos vaõs serão mostrados, nomeadamente, o vão entre os pilares B e 7 do eixo Central (ver Figura 4-6).

Para aplicação dos métodos FDD, PSDT PSDTM-SVD foram calculados os espectros da densidade espectral de potência, usando o método de Welch, no qual foi definida uma janela de Hanning com o tamanho de $2^{11}$ dados, com uma sobreposição de $50 \%$ e um tamanho da transformada de Fourier de $2^{11}$.

Realizando uma revisão dos valores singulares das matrizes de transmissibilidade no método PSDTM-SVD e analisando as frequências que convergem para zero na decomposição pode-se obter uma análise preliminar das frequências naturais da ponte. Assim, como exemplo na Figura 4-9, é apresentado os valores singulares de três matrizes de transmissibilidade para a configuração de acelerômetros 1 (setup 1) sobre o eixo Central da ponte, no vão B-7. A Figura 4-9 mostra uma convergência para zero de diferentes frequências de ressonância na faixa de 0 a $100 \mathrm{~Hz}$, mas com maior importância na faixa de 0 a $20 \mathrm{~Hz}$. 


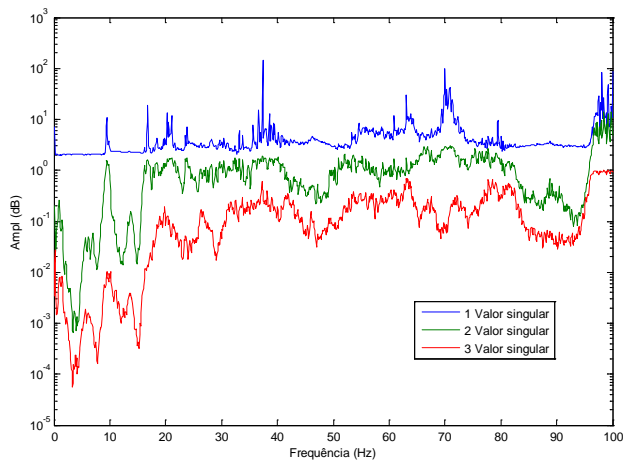

a.)

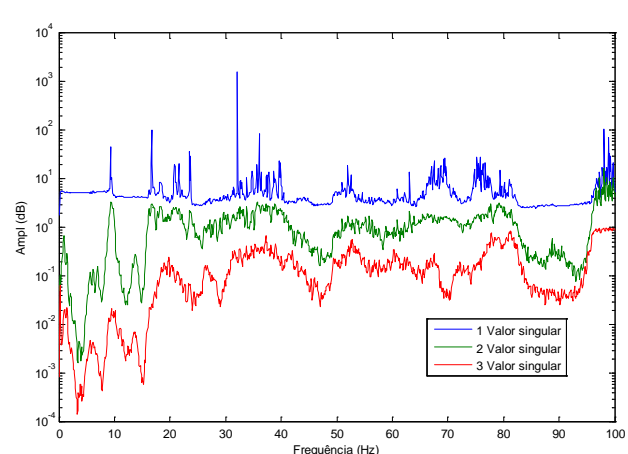

b.)

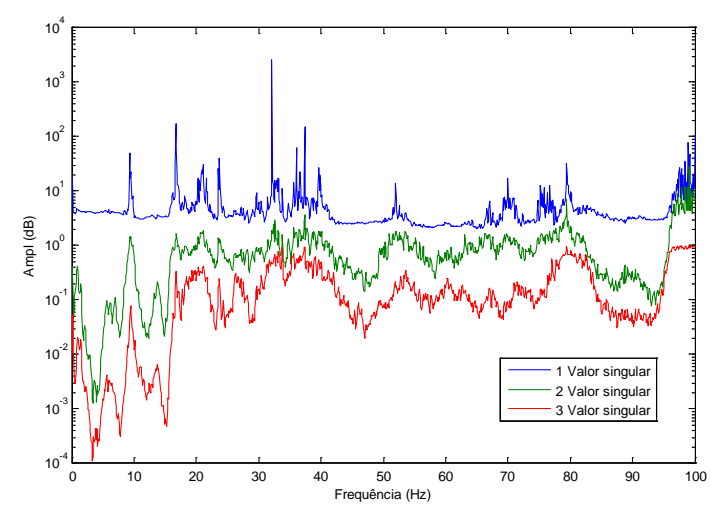

c.)

Figura 4-9 Decomposição em valores singulares das matrizes de transmissibilidade para a configuração 1 (setup 1) no eixo central da ponte, no vão B-7.

Os resultados do método proposto PSTM-SVD foram comparados com aqueles dos métodos FDD e PSDT para as três configurações de acelerômetros sobre o eixo Central da ponte, no vão B-7, os quais são mostrados nas Figura 4-10, 4-11 e 4-12. As frequências de ressonância podem ser extraídas a partir dos picos dos gráficos nas Figura 4-10, 4-11 e 4-12. A faixa de frequência foi truncada até um valor de $40 \mathrm{~Hz}$ para uma melhor visualização das frequências de interesse. Os picos que foram claramente visíveis na faixa de 0 a $20 \mathrm{~Hz}$ foram selecionados como frequências de ressonância da ponte. As frequências identificadas para as três configurações de acelerômetros são resumidas na Tabela 4-4. Uma média para todas as configurações de acelerômetros são mostrados nas colunas 11,12 e 13 da Tabela 4-4. Além disso, a Tabela 4-4 mostra que a maioria das frequências identificadas na faixa de 0 a $20 \mathrm{~Hz}$ são praticamente as mesmas para as diferentes configurações de acelerômetros, 0 que indica uma correta identificação das frequências de ressonância da ponte. 


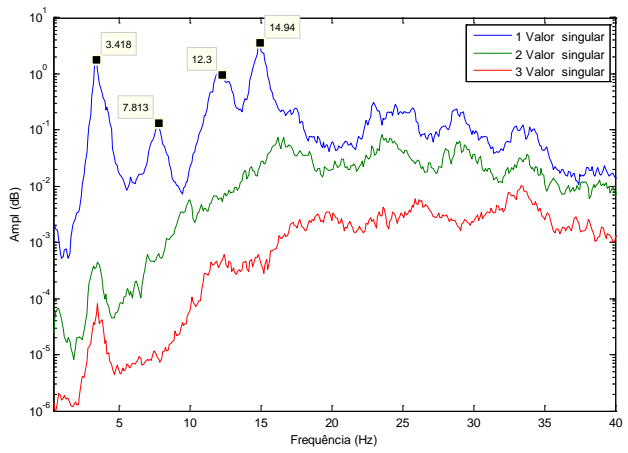

a)

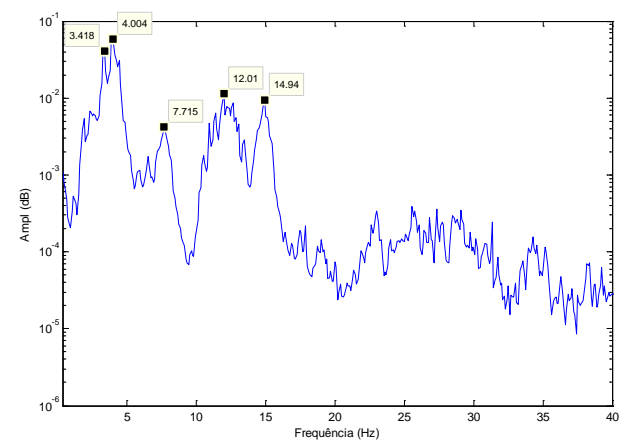

b)

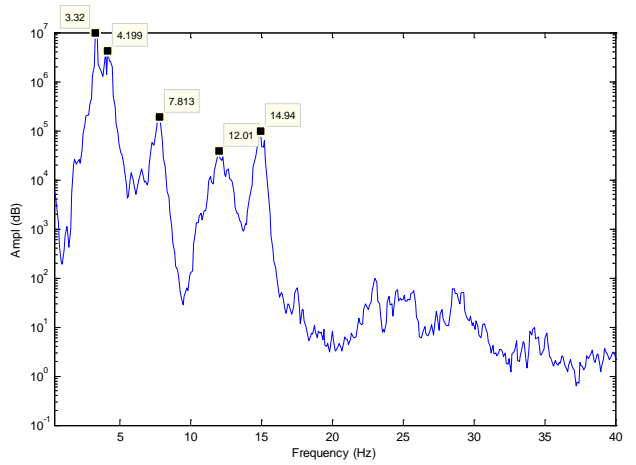

c.)

Figura 4-10 Resultados dos métodos a) FDD, b) PSDT, c) PSDTM-SVD para a configuração 1 (setup 1 ) sobre o eixo Central da ponte, no vão B-7.

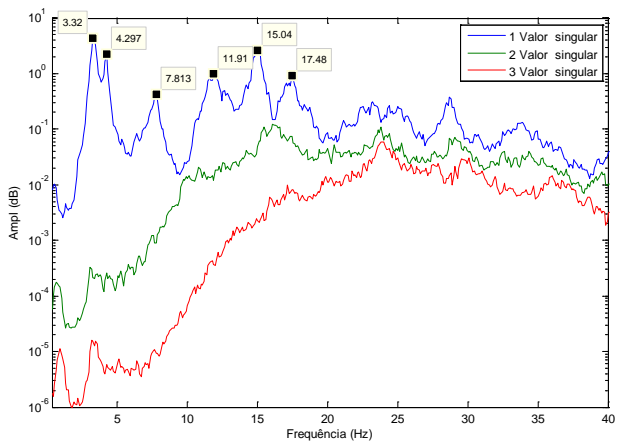

a)

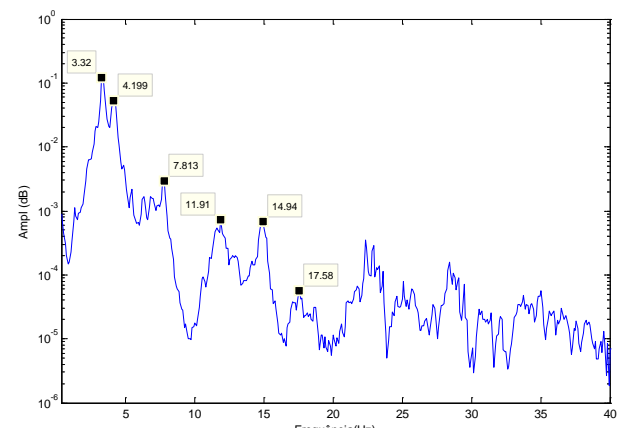

b)

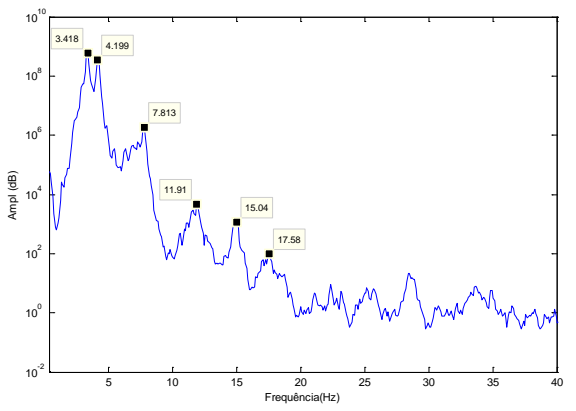

c)

Figura 4-11 Resultados dos métodos a) FDD, b) PSDT, c) PSDTM-SVD para a configuração 2 (setup 2) sobre o eixo Central da ponte, no vão B-7. 


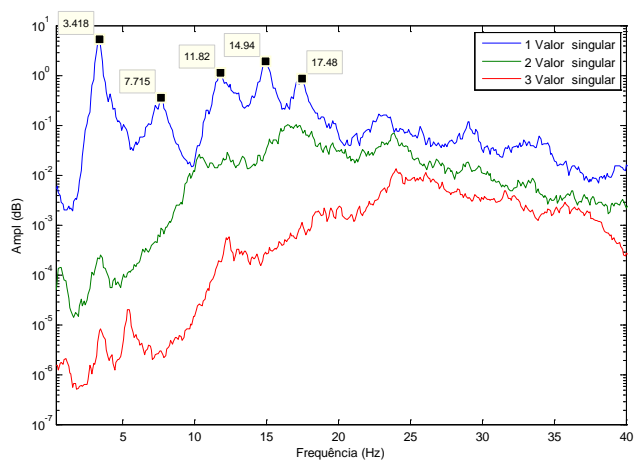

a)

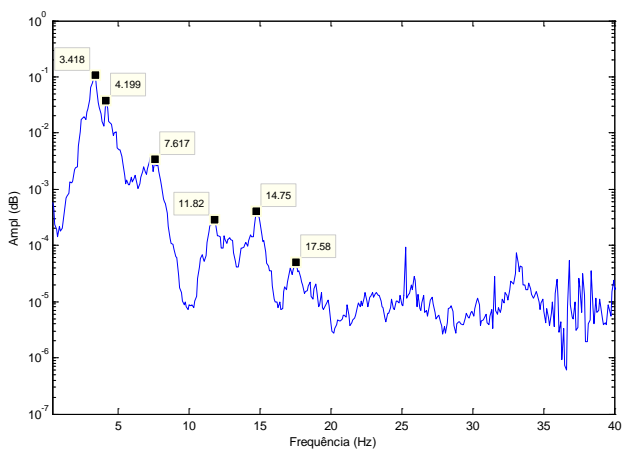

b)

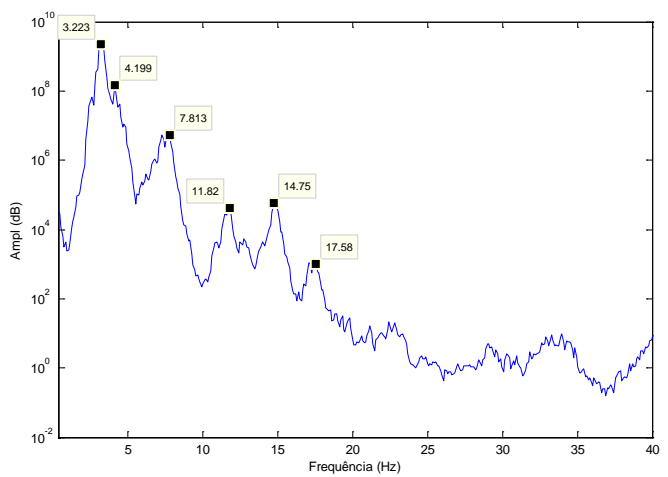

c)

Figura 4-12 Resultados dos métodos a) FDD, b) PSDT, c) PSDTM-SVD para a configuração 3 (setup 3 ) sobre o eixo Central da ponte, no vão B-7.

Tabela 4-4 Frequências naturais identificadas $(\mathrm{Hz})$ a partir das diferentes configurações de acelerômetros (Setups) sobre o eixo Central da ponte, no vão B-7.

\begin{tabular}{|c|c|c|c|c|c|c|c|c|c|c|c|c|}
\hline \multirow[b]{2}{*}{ Modos } & \multicolumn{3}{|c|}{ Setup 1} & \multicolumn{3}{|c|}{ Setup 2} & \multicolumn{3}{|c|}{ Setup 3} & \multicolumn{3}{|c|}{ Média setups } \\
\hline & FDD & PSDT & PSDTM-SVD & FDD & PSDT & PSDTM-SVD & FDD & PSDT & PSDTM-SVD & FDD & PSDT & PSDTM-SVD \\
\hline 1 & 3.418 & 3.418 & 3.320 & 3.320 & 3.320 & 3.418 & 3.418 & 3.418 & 3.223 & 3.385 & 3.385 & 3.320 \\
\hline 2 & \# & 4.004 & 4.199 & 4.297 & 4.199 & 4.199 & \# & 4.199 & 4.199 & 4.297 & 4.134 & 4.199 \\
\hline 3 & 7.813 & 7.715 & 7.813 & 7.813 & 7.813 & 7.813 & 7.715 & 7.617 & 7.813 & 7.780 & 7.715 & 7.813 \\
\hline 4 & 12.300 & 12.010 & 12.010 & 11.910 & 11.910 & 11.910 & 11.820 & 11.820 & 11.820 & 12.010 & 11.913 & 11.913 \\
\hline 5 & 14.940 & 14.940 & 14.940 & 15.040 & 14.940 & 15.040 & 14.940 & 14.750 & 14.750 & 14.973 & 14.877 & 14.910 \\
\hline 6 & \# & \# & \# & 17.480 & 17.580 & 17.580 & 17.480 & 17.580 & 17.580 & 17.480 & 17.580 & 17.580 \\
\hline
\end{tabular}

Uma vez que as frequências de ressonância são identificadas, as formas modais podem ser calculadas com o método PSDTM-SVD. As formas modais identificadas com o método PSDTM-SVD são mostradas na Figura 4-13 e comparadas com os resultados dos métodos FDD e PSDT, mostrando concordância entre os métodos. Note-se na Figura 4-13 a existência de formas modais idênticas para diferentes frequências de ressonância. Essas formas modais repetidas na superestrutura da ponte são devidas à existência de formas 
modais bidimensionais. Umas medições de vibração em ambos os sentidos transversal e longitudinal da ponte são necessárias para definir completamente as formas modais, especialmente neste tipo de ponte que apresenta uma largura grande da placa. Nesse estudo realizado não foi possível obter medições transversais devido ao tipo de equipamento utilizado, o qual é conectado com cabos que podem ser afetados pela passagem dos veículos. Uma descrição mais detalhada do comportamento dinâmico da ponte pode ser encontrada em (Araujo, Maldonado e Cho, 2011). Assim, finalmente é demostrado nesta aplicação com dados de vibrações reais que a metodologia proposta tem a capacidade de identificar parâmetros modais.
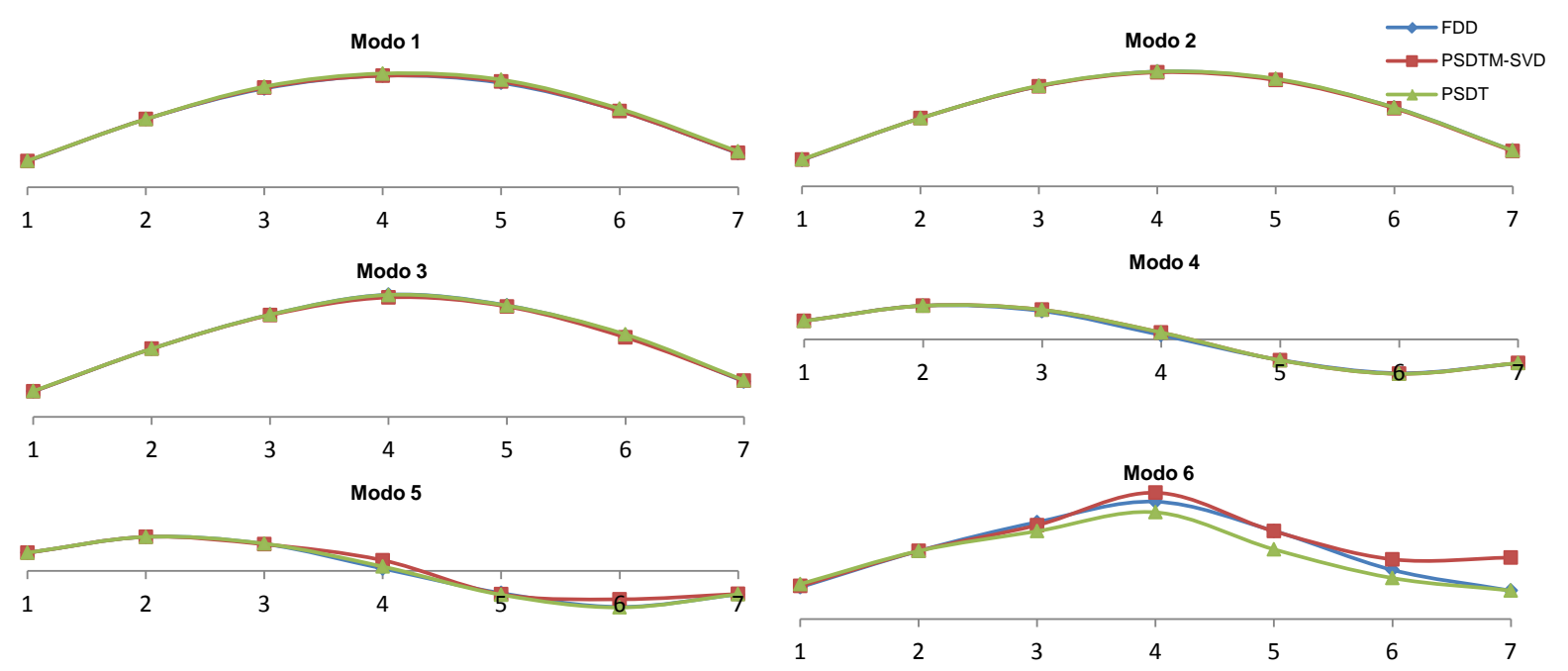

Figura 4-13 Comparação das formas modais identificadas com os métodos FDD, PSDT e PSDTM-SVD sobre o eixo Central da ponte, no vão B-7.

\subsection{CONSIDERAÇÕES FINAIS.}

O método PSDTM-SVD proposto neste capítulo é usado para identificar frequências naturais e modos de vibração do sistema sem o conhecimento da entrada ao sistema. $O$ método utiliza o conceito principal do método PSDT, para criar as matrizes de transmissibilidade, que nas linhas convergem à mesma relação de amplitudes de modos de vibração nos pólos do sistema, depois é aplicada uma decomposição de valores singulares que é usada para a identificação de parâmetros modais. Similar ao método PSDT, o PSDTM-SVD proposto tem uma independência do espectro da excitação, portanto, pode ser aplicado para qualquer tipo de carregamento de entrada (ruído branco ou ruído colorido), se somente se as frequências do sistema são excitadas. Também, as frequências naturais e formas modais podem ser extraídas a partir de uma única condição de carga ao invés de diferentes condições de carga como proposto em anteriores metodologias que usam transmissibilidade. No entanto, o PSDTM-SVD reduz ainda mais o risco de estabelecer 
frequências naturais que não são do sistema, em comparação com o PSDT que utiliza uma média normalizada de funções de subtração inversa entre funções de transmissibilidade, que podem conter picos de ressonancia não relacionadas às frequencias naturais.

O PSDTM-SVD foi implementado para realizar uma análise modal com unicamente dados da resposta sobre uma estrutura tipo viga, a qual foi sujeita a uma excitação com uma frequência predominante. Duas distribuções de carregamento distintas foram analisadas. A partir dos resultados da análise das respostas das duas distribuções de carregamento, pode-se observar que o PSDTM-SVD estabeleceu adequadamente as frequências naturais e modos de vibração do sistema sem a introdução de modos espúrios em comparação com os metodos FDD e PSDT. Além disso, o método proposto foi usado para analisar os dados de um ensaio de vibração sobre uma ponte. Os resultados dessa aplicação real demonstrou que o método proposto pode ser utilizado para identificar os parâmetros modais de uma estrutura sob condições de carga operacional. 



\section{CAPÍTULO 5. TRANSMISSIBILIDADE MULTIVARIÁVEL COM DIFERENTES REFERÊNCIAS DAS SAÍDAS.}

Tradicionalmente o conceito de transmissibilidade é tratado no domínio da frequência, como uma relação entre duas respostas, no entanto, existem trabalhos como Varoto e Mcconnell (1998) e Liu et al. (1998) que tentaram estender o conceito para uma relação de múltiplas respostas. Assim, finalmente uma extensão do conceito de transmissibilidade para múltiplas respostas em sistemas de múltiplos graus de liberdade excitados harmonicamente foi dada por Riberio, Silva e Maia (2000). Igualmente, essa generalização foi estendida para sistemas com excitações aleatórias em Fontul et al. (2004) obtendo uma matriz de transmissibilidade multivariável idêntica à das excitações harmônicas. Esses conceitos de transmissibilidade escalar e multivariável são abordados no Capítulo 2.

A maioria de métodos de identificação modal com transmissibilidade usa como base a transmissibilidade escalar (relação de duas respostas) para a avaliação das funções de transmissibilidade. Incluindo o método proposto no Capítulo 4. Uma exceção do uso da transmissibilidade escalar é o método proposto em Devriendt, De Sitter e Guillaume (2010), o qual usa a transmissibilidade multivariável sob diferentes condições de carga para avaliar parâmetros modais. No entanto, em Devriendt, De Sitter e Guillaume (2010) foi demostrado que as funções de transmissibilidade multivariável para diferentes condições de carga não se cruzam na ressonância como acontece nas funções de transmissibilidade escalar, portanto, não se podem usar diretamente para a avaliação de parâmetros modais. Dessa forma, que em Devriendt e De Sitter e Guillaume (2010) foi estabelecida uma relação entre as funções de transmissibilidade multivariável e as escalares para dar solução ao problema. A partir dessa relação são estimadas umas funções chamadas de "pseudo-funções de transmissibilidade escalar", as quais se cruzam na ressonância para diferentes condições de carga. Portanto, as obtidas "pseudo-funções de transmissibilidade escalar" podem ser usadas similarmente como as funções de transmissibilidade escalar para obter parâmetros modais.

Inspirado no trabalho de Yan e Ren (2012), no qual se demostrou que as escalares PSDT para diferentes referências da saída convergem à mesma relação dos modos de vibração na ressonância, neste capítulo pretende-se estender o conceito da escalar PSDT para um conceito de multivariável PSDT com diferentes referências da saída. A multivariável 
PSDT permite relacionar múltiplas saídas ao invés de só duas. A multivariável PSDT com diferentes referências de saída é uma matriz que relaciona uma matriz de densidade espectral cruzada das respostas nas coordenadas $Z$ e $U$ com uma matriz de densidade espectral cruzada das respostas nas coordenadas Z e K. As coordenas em Z são chamadas como referências da saída. Uma importante propriedade surge a partir da multivariável PSDT, na qual diferentes matrizes multivariável PSDT com as mesmas coordenadas $\mathrm{K}$ e $\mathrm{U}$, mas com diferentes referências da saída $Z$ convergem para a mesma matriz nos pólos do sistema. Essa propriedade pode ser usada para fornecer uma alternativa para a identificação de parâmetros dinâmicos, como é proposto neste capítulo. O objetivo é construir uma única matriz com a informação a partir de diferentes matrizes multivariáveis PSDTs com diferentes referências da saída $Z$ com as mesmas coordenas $\mathrm{K}$ e $\mathrm{U}$. Esta única matriz tem uma singularidade no pólos do sistema. Dessa forma, uma pseudoinversa (Moore-Penrose) "modificada" pode ser aplicada para determinar frequências naturais amortecidas e taxas de amortecimento. As formas de vibração do sistema podem ser calculadas com o primeiro vetor singular à esquerda da matriz nos pólos do sistema, mas uma condição das coordenadas $\mathrm{K}$ e $\mathrm{U}$ devem ser satisfeitas. Finalmente, o método para a identificação de parâmetros modais usando multivariável PSDT foi verificado em uma análise numérica de uma viga e com dados reais de vibração de uma ponte, como também foi mostrado no Capítulo 4.

\subsection{FUNÇÕES DE TRANSMISSIBILIDADE MULTIVARIÁVEL COM DIFERENTE REFERÊNCIA DA SAÍDA.}

As funções de transmissibilidade multivariável com diferentes referências da saída podem ser representadas a partir de definir as seguintes variáveis: um vetor coluna $F_{A}$ que contém as magnitudes das forças aplicadas ao sistema nas coordenadas $A$, um vetor coluna $X_{K}$ com amplitudes das respostas nas coordenadas $K$, um vetor coluna $X_{U}$ com amplitudes das respostas nas coordenadas $U$ e um vetor coluna $X_{Z}$ com as amplitudes das respostas nas coordenadas $Z$. O vetor $X_{Z}$ é definido como o vetor de referência da saída. Essas variáveis definem as seguintes relações.

$$
\begin{aligned}
& \left\{X_{u}(i \omega)\right\}=\left|H_{x_{u} f_{a}}(i \omega)\right|\left\{F_{a}(i \omega)\right\} \text { ou }\left\{X_{u}(i \omega)\right\}^{T}=\left\{F_{a}(i \omega)\right\}^{T}\left[H_{x_{u} f_{a}}(i \omega)\right]^{T} \\
& \left\{X_{k}(i \omega)\right\}=\left|H_{x_{k} f_{a}}(i \omega)\right|\left\{F_{a}(i \omega)\right\} \text { ou }\left\{X_{k}(i \omega)\right\}^{T}=\left\{F_{a}(i \omega)\right\}^{T}\left[H_{x_{k} f_{a}}(i \omega)\right]^{T}
\end{aligned}
$$

Em que $H_{x_{u} f_{a}}(i \omega)$ e $H_{x_{k} f_{a}}(i \omega)$ são as funções de transferência relacionando as coordenadas $U-A$, e $K-A$, respectivamente. Multiplicando as Equações (5-1) e (5-2) pelo vetor de referência $X_{Z}$ conjugado, e considerando que a correlação entre as duas respostas 
se relaciona com a convolução, como $R_{x x}(k)=x(-n) \times x(n)$ é representada no domínio da frequência em densidade espectral, como $S_{x x}(i \omega)=x(i \omega)^{*} \times x(i \omega)$, podem-se estabelecer nas Equações (6-1) e (6-2) as seguintes relações.

$$
\begin{aligned}
& \left\{X_{z}(i \omega)\right\}^{*}\left\{X_{u}(i \omega)\right\}^{T}=\left\{X_{z}(i \omega)\right\}^{*}\left\{F_{a}(i \omega)\right\}^{T}\left[H_{X_{u} f_{a}}(i \omega)\right]^{T} \\
& \left\{X_{z}(i \omega)\right\}^{*}\left\{X_{k}(i \omega)\right\}^{T}=\left\{X_{z}(i \omega)\right\}^{*}\left\{F_{a}(i \omega)\right\}^{T}\left[H_{x_{k} f_{a}}(i \omega)\right]^{T}
\end{aligned}
$$

$\mathrm{Ou}$

$$
\begin{aligned}
& \left.\left[S_{x_{z} x_{u}}(i \omega)\right]=\left[S_{x_{z} f_{a}}(i \omega)\right] H_{x_{u} f_{a}}(i \omega)\right]^{T} \\
& \left.\left[S_{x_{z} x_{k}}(i \omega)\right]=\left[S_{x_{z} f_{a}}(i \omega)\right] H_{x_{k} f_{a}}(i \omega)\right]^{T}
\end{aligned}
$$

Das Equações (5-5) e (5-6) verifica-se que

$$
\begin{aligned}
& {\left[S_{x_{z} x_{u}}(i \omega)\right]^{T}=\left[H_{x_{u} f_{a}}(i \omega) \llbracket S_{x_{z} f_{a}}(i \omega)\right]^{T}} \\
& {\left[S_{x_{z} x_{k}}(i \omega)\right]^{T}=\left[H_{x_{k} f_{a}}(i \omega) \llbracket S_{x_{z} f_{a}}(i \omega)\right]^{T}}
\end{aligned}
$$

Combinando a Equações (5-7) e (5-8), segue que.

$$
\begin{aligned}
& \left.\left[S_{x_{z} x_{u}}(i \omega)\right]^{T}=\left[H_{x_{u} f_{a}}(i \omega)\right] H_{x_{k} f_{a}}(i \omega)\right]^{+}\left[S_{x_{z} x_{k}}(i \omega)\right]^{T} \\
& {\left[S_{x_{z} x_{u}}(i \omega)\right]^{T}=\left[T^{z}{ }_{x_{u} x_{k}}(i \omega)\right]_{z}\left[S_{x_{z} x_{k}}(i \omega)\right]^{T}}
\end{aligned}
$$

A expressão (5-10) relaciona a matriz de densidade espectral cruzada entre as respostas $Z$ e $U$ com a matriz de densidade espectral cruzada entre as respostas $Z$ e $K$. Pode-se notar na Equação (5-10), que se Z=K, a matriz de transmissibilidade será a mesma representada para as vibrações aleatórias e harmônicas como mostrado no Capítulo 2. Assim, as funções de transmissibilidade multivariável com diferentes referências da saída podem-se representar, como.

$$
\left[T^{z}{ }_{x_{u} x_{k}}(i \omega)\right]=\left[S_{x_{z} x_{u}}(i \omega)\right]^{T}\left[\left[S_{x_{z} x_{k}}(i \omega)\right]^{T}\right]^{+}
$$

A matriz de transmissibilidade mostrada na Equação (5-11) é dada por funções de transmissibilidade internas, como. 


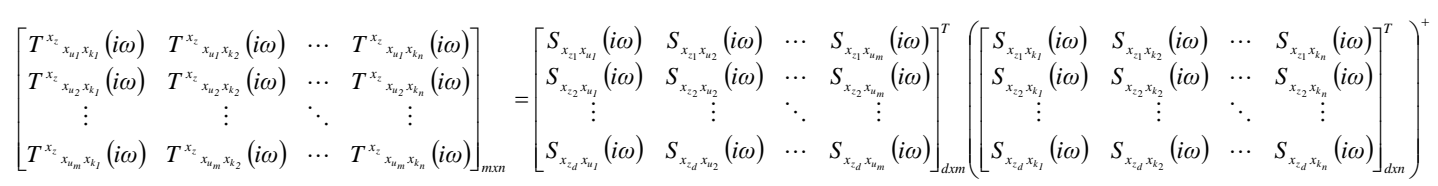

Em que, $m, n$ e $d$ são o número total das coordenadas de $U, K, Z$ respectivamente.

\subsection{PROPRIEDADE DA PSDT MULTIVARIÁVEL.}

A formulação apresentada permite a extração de uma propriedade importante das matrizes de transmissibilidade com diferentes referências da saída, $\left[T^{z}{ }_{x_{u} x_{k}}(i \omega)\right]$. Essa propriedade pode-se estabelecer da seguinte forma: as matrizes de transmissibilidade multivariável com as mesmas coordenadas $\mathrm{Ke} \mathrm{U}$, mas com diferentes referências da saída $Z$, convergem para a mesma amplitude quando se aproximam aos pólos do sistema. Esta propriedade pode ser derivada a partir da Equação (3-39), representada como.

$\lim _{i \omega \rightarrow i \omega_{l}} S_{x_{k} x_{j}}(i \omega) \cong \varphi^{*}{ }_{k l} D\left(i \omega_{l}\right) \varphi_{j l} \operatorname{com} D\left(i \omega_{l}\right)=\sum_{r=1}^{N} \sum_{s=1}^{N} \hat{H}_{z_{l} f_{r}}^{*}\left(i \omega_{l}\right) S_{f_{r} f_{s}}\left(i \omega_{l}\right) \hat{H}_{z_{l} f_{s}}\left(i \omega_{l}\right)$

E definindo um vetor $X_{U}$ do tamanho $\mathrm{m \times 1}$, um vetor $X_{K}$ do tamanho $\mathrm{nx1}$ e diferentes referências da saída $X_{Z}$, todas do tamanho $1 \times 1$. Substituindo (5-13) na Equação (5-11) o limite da transmissibilidade multivariável, $\left[T^{z}{ }_{x_{u} x_{k}}(i \omega)\right]$, nos pólos do sistema $i \omega_{l}$ com diferentes referências das saídas $X_{Z_{i}}$ é definida como.

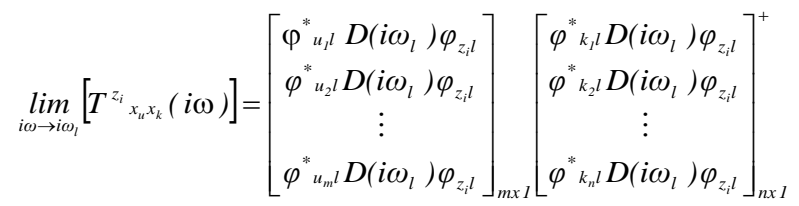

$$
\begin{aligned}
& =D\left(i \omega_{l}\right) \varphi_{z_{l} l}\left[\begin{array}{c}
\varphi^{*}{ }_{u_{1} l} \\
\varphi^{*}{ }_{u_{2} l} \\
\vdots \\
\varphi^{*}{ }_{u_{m} l}
\end{array}\right]_{m \times 1} \frac{1}{D\left(i \omega_{l}\right) \varphi_{z_{l} l} \sum_{j=1}^{n}\left|\varphi^{*}{ }_{k, l}\right|^{2}}\left[\begin{array}{llll}
\varphi_{k_{1} l} & \varphi_{k_{2} l} & \cdots & \varphi_{k_{n} l}
\end{array}\right]_{1 \times n}
\end{aligned}
$$

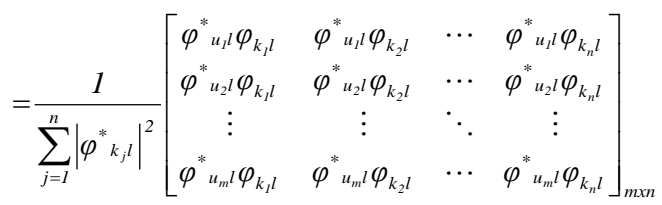

Na Equação (5-14), o limite no pólo do sistema para a transmissibilidade multivariável $\left[T^{x_{z_{i}}} x_{x_{i} x_{k}}(i \omega)\right]$ é o mesmo qualquer seja a coordenada selecionada para $X_{z_{i}}$.Dessa forma, diferentes matrizes de transmissibilidade, $\left[T^{x_{z_{i}}}{ }_{{ }_{u} x_{k}}(i \omega)\right]$ podem surgir pela variação das referências da saída $X_{Z_{i}}$ de $\mathrm{i}=1$ até $\mathrm{L}$, em que $\mathrm{L}$ é o número total de pontos de medição. Estas matrizes de transmissibilidade nos pólos do sistema convergem para Equação (5-14). 
A independência do espectro da entrada ao sistema na matriz de transmissibilidade proposta é outra importante característica que pode ser determinada a partir da Equação (5-14). O termo $D\left(i \omega_{l}\right)$, o qual contém os espectros das entradas $S_{f_{r} f_{s}}\left(i \omega_{l}\right)$, é eliminado na Equação (5-14). De fato, a eliminação do espectro da entrada ao sistema $\left[\mathbf{S}_{\mathbf{x}_{\mathbf{z}} \mathbf{f}_{\mathbf{a}}}(\mathbf{i} \omega)\right]$ acontece para todos os valores de $i \omega$, já que $\left[\mathbf{S}_{\mathbf{x}_{2} \mathbf{f}_{\mathbf{a}}}(\mathbf{i} \omega)\right]$ é eliminada entre as equações (5-7) e (5-8). No entanto, na Equação (5-9) as funções de transferência $\left[\mathbf{H}_{\mathbf{x}_{\mathbf{u}} \mathbf{f}_{\mathbf{a}}}(\mathbf{i} \omega)\right\rfloor$ e $\left[\mathbf{H}_{\mathbf{x}_{\mathbf{k}} \mathbf{f}_{\mathbf{a}}}(\mathbf{i} \omega)\right]$ mostram que a matriz de transmissibilidade proposta depende da posição das cargas. Desta forma, pode ser verificado que a matriz de transmissibilidade proposta depende somente da posição das cargas e não do espetro da excitação.

\subsection{IDENTIFICAÇÃO DE PARÂMETROS MODAIS USANDO MULTIVARIÁVEL PSDT.}

A propriedade como mostrada na Equação (5-14) é adotada para prover uma alternativa na análise modal operacional. Os parâmetros modais podem ser identificados pela combinação de diferentes matrizes de transmissibilidade multivariável com diferentes referências da saída a partir de uma única condição de carga. O objetivo é construir uma única matriz com toda a informação de diferentes matrizes de transmissibilidade multivariável contendo diferentes referências da saída $\mathrm{Z}$ e as mesmas coordenadas $\mathrm{K}$ e $\mathrm{U}$. Dessa maneira, a matriz é definida como.

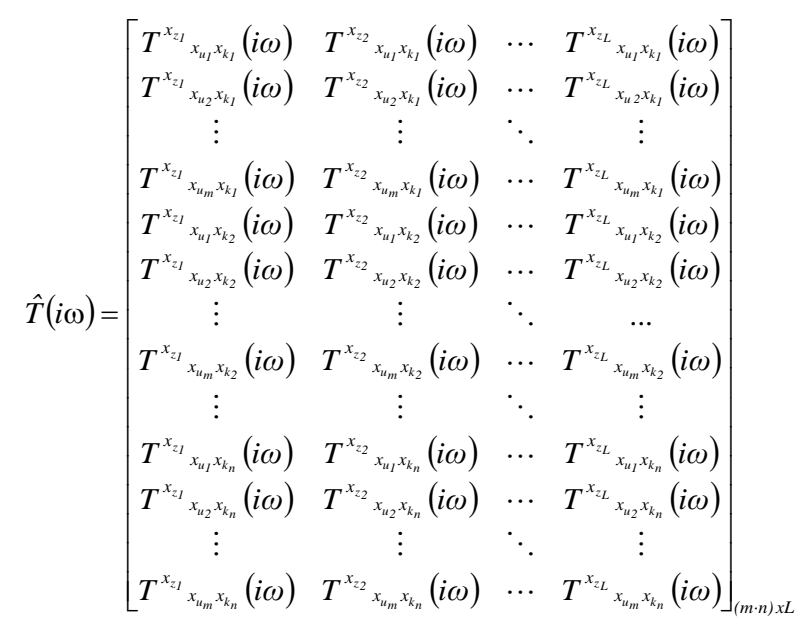

$\mathrm{Na}$ Equação (5-15), as colunas contêm as funções de transmissibilidade com as mesmas coordenadas $U$ e $K$, mas com diferentes referências da saída $Z$, as quais variam de 1 até L. As linhas correspondem a diferentes funções de transmissibilidade da (5-12) 
contendo a mesma referência da saída Z. A partir da propriedade definida na Equação (5-15) é possível definir uma matriz na qual nos pólos do sistema converge na seguinte matriz.

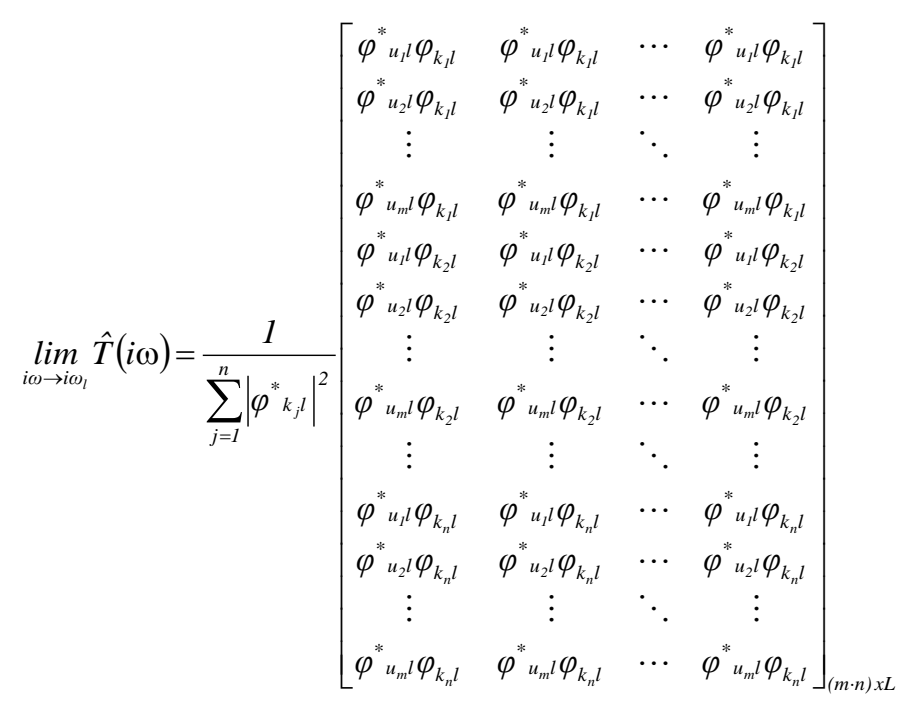

O posto da matriz proposta na Equação (5-15) é igual a 1 quando a matriz se aproxima no pólos do sistema, contendo colunas que são linearmente dependentes, como mostrado na Equação (5-16). Isso indica que os valores singulares da matriz na Equação (5-15) converge para zero no pólos do sistema a partir do segundo valor singular $\sigma_{2}\left(i \omega_{l}\right)=\sigma_{3}\left(i \omega_{l}\right)=\ldots .=\sigma_{r}\left(i \omega_{l}\right)=0$. Portanto, a matriz na Equação (5-15) chega a ser uma matriz singular quando ela se aproxima nos pólos do sistema. A singularidade de essa matriz pode ser avaliada usando a inversa generalizada Moore-Penrose. Esta técnica foi usada previamente por Devriendt et al. (2013) para quantificar a singularidade de uma matriz contendo funções de transmissibilidade obtidas de diferentes condições de carga. Similarmente, a inversa generalizada Moore-Penrose é usada aqui para determinar os pólos do sistema, mas com modificações.

Primeiro, a inversa Moore-Penrose da Equação (5-15) é obtida usando a decomposição dos valores singulares da seguinte forma.

$$
\begin{aligned}
{[\hat{T}(i \omega)]^{+}=} & \sum_{i=1}^{r} \frac{1}{\sigma_{i}(i \omega)}\left\{\hat{V}(i \omega)_{i}\right\} \cdot\left\{\hat{U}(i \omega)_{i}\right\}^{H} \\
& =\frac{1}{\sigma_{1}(i \omega)}\left\{\hat{V}(i \omega)_{1}\right\} \cdot\left\{\hat{U}(i \omega)_{1}\right\}^{H}+\frac{1}{\sigma_{2}(i \omega)}\left\{\hat{V}(i \omega)_{2}\right\} \cdot\left\{\hat{U}(i \omega)_{2}\right\}^{H}+\ldots+\frac{1}{\sigma_{r}(i \omega)}\left\{\hat{V}(i \omega)_{r}\right\} \cdot\left\{\hat{U}(i \omega)_{r}\right\}^{H} \\
& =\left[\hat{T}(i \omega)_{1}\right]^{+}+\left[\hat{T}(i \omega)_{2}\right]^{+}+\ldots+\left[\hat{T}(i \omega)_{r}\right]^{+}
\end{aligned}
$$

Em que, $\hat{U}_{i}$ é um vetor singular a esquerda da matriz $\hat{T}$ do tamanho $(m n) x 1, \hat{V}_{i}$ é um vetor singular à direita da matriz $\hat{T}$ do tamanho $L x 1, \sigma_{i}$ é um número real com os valores 
singulares da matriz $\hat{T}$ e $r$ é o número total de valores singulares. Se os valores singulares nos pólos do sistema da matriz $\hat{T}$ convergem para zero a partir do segundo valor singular, então os valores inversos dos valores singulares tendem ao infinito $1 / \sigma_{2}\left(i \omega_{l}\right)=1 / \sigma_{3}\left(i \omega_{l}\right)=\ldots .=1 / \sigma_{r}\left(i \omega_{l}\right)=\infty$.

Se os valores singulares inversos na Equação (5-17), a partir do segundo valor singular tendem ao infinito nos pólos do sistema, $1 / \sigma_{2}\left(i \omega_{l}\right)=1 / \sigma_{3}\left(i \omega_{l}\right)=\ldots .=1 / \sigma_{r}\left(i \omega_{l}\right)=\infty$, então as matrizes de $\hat{T}(i \omega)_{2}^{+}$até $\hat{T}(i \omega)_{r}^{+}$também convergem ao infinito no pólos do sistema, $i \omega_{l}$. Portanto, cada função das matrizes de $\hat{T}(i \omega)_{2}^{+}$até $\hat{T}(i \omega)_{r}^{+}$têm pólos iguais aos pólos reais do sistema. No entanto, duas modificações são propostas na Equação (5-17). A primeira é eliminar a contribuição do primeiro valor singular, e a segunda é aplicar uma somatória truncada na Equação (5-17). A soma truncada na Equação (5-17) só utiliza os primeiros k valores singulares (em que, $\mathrm{k}<\mathrm{r}$ ) e elimina os menores valores singulares.

A soma na Equação (5-17) é truncada devido a que a matriz de transmissibilidade multivariável com diferentes referências da saída, como foi definida na Equação (5-11) tem um máximo posto igual ao número de cargas não correlacionadas de entrada ao sistema (Leclere, Roozen e Sandier, 2014). Portanto, a Equação (5-15) que é construída a partir de diferentes matrizes de transmissibilidade multivariável também tem o mesmo máximo posto. Dessa forma, o número de valores singulares $k$, que deve ser selecionado não pode ser maior que o número de cargas de entrada. Os valores singulares entre k e r são os termos mais significativos da Equação (5-17), devido a que seu fator de magnificação $1 / \sigma_{i}(i \omega)$ é maior. No entanto, os menores valores singulares representam o ruído. Portanto, se na Equação (5-17) são selecionados os maiores valores singulares, então a matriz $\hat{T}(i \omega)^{+}$é representada por uma matriz inversa de posto k que contém menor ruído (Hansen, 1987). Nesse sentido, uma média de todos os valores singulares em i $\omega$ para cada valor singular é usada como critério para selecionar o maior valor singular k na Equação (5-17), como será mostrado depois no exemplo numérico deste capítulo.

Uma nova matriz, chamada como $\hat{T}(i \omega)^{++}$é definida considerando as anteriores modificações na Equação (5-17). 


$$
\begin{aligned}
{[\hat{T}(i \omega)]^{++} } & =\sum_{i=2}^{k} \frac{1}{\sigma_{i}(i \omega)}\left\{\hat{V}(i \omega)_{i}\right\} \cdot\left\{\hat{U}(i \omega)_{i}\right\}^{H} \\
& =\left[\hat{T}(i \omega)_{2}\right]^{+}+\left[\hat{T}(i \omega)_{3}\right]^{+}+\ldots+\left[\hat{T}(i \omega)_{k}\right]^{+}
\end{aligned}
$$

Como uma consequência, nos pólos do sistema de $\hat{T}(i \omega)^{++}$fica.

$$
\lim _{i \omega \rightarrow i \omega_{l}}[\hat{T}(i \omega)]^{++}=\left[\begin{array}{ccccccccccccc}
\infty & \infty & \cdots & \infty & \infty & \infty & \cdots & \infty & \cdots & \infty & \infty & \cdots & \infty \\
\infty & \infty & \cdots & \infty & \infty & \infty & \cdots & \infty & \cdots & \infty & \infty & \cdots & \infty \\
\vdots & \vdots & \ddots & \vdots & \vdots & \vdots & \ddots & \vdots & \ddots & \vdots & \vdots & \ddots & \vdots \\
\infty & \infty & \cdots & \infty & \infty & \infty & \cdots & \infty & \cdots & \infty & \infty & \cdots & \infty
\end{array}\right]_{L \times(m \cdot n)}
$$

De acordo com Devriendt et al. (2013) em uma matriz com a propriedade mostrada na Equação (5-19), é possível aplicar qualquer método paramétrico no domínio da frequência para identificar os pólos do sistema. Consequentemente, o método PolyMAX (Peeters et al., 2004) apresentado no Capítulo 3 foi usado para modelar a matriz proposta, $\hat{T}(i \omega)^{++}$, e estimar as frequências de ressonância amortecidas e as taxas de amortecimentos.

Uma vez os pólos do sistema são identificados, é possível avaliar os modos de vibração usando o primeiro vetor singular à esquerda $\hat{U}(i \omega)$ na frequência natural $i \omega_{l}$. No entanto, uma condição deve ser satisfeita para determinar os modos de vibração completos: as coordenadas em $U$ devem conter todos os $L$ pontos de medição, e as coordenas em $K$ devem ter somente um valor, inclusive para qualquer coordenada de $\mathrm{K}$ que coincida com uma coordenada de U. Seguindo está condição, é possível determinar que o primeiro vetor singular $\hat{U}(i \omega)_{1}$ converge nos pólos do sistema para a seguinte relação.

$$
\hat{U}\left(i \omega_{l}\right)_{1}=\frac{1}{\left(\left.\sum_{i}^{L} \frac{\left.\left|\varphi^{*} u_{i}\right|^{2}\right|^{2}}{\mid \varphi^{*} u_{L} l}\right|^{2}\right)^{1 / 2} \varphi^{*} u_{L} l}\left(\left[\begin{array}{llll}
\varphi^{*} u_{1} l & \varphi^{*} u_{2} l & \ldots & \varphi^{*} u_{L} l
\end{array}\right]_{1 \times L}\right)^{T}
$$

Se a condição anterior é considerada, então a Equação (5-20) mostra que o primeiro vetor singular à esquerda $\hat{U}(i \omega)_{1}$ nos pólos do sistema é proporcional ao modo de vibração do sistema contendo todos os pontos de medição $L$.

\subsection{EXEMPLO NUMÉRICO}

Para demonstrar o desempenho na identificação de parâmetros modais do método proposto neste capítulo foi usado um sistema linear invariante no tempo. O sistema usado é o mesmo usado no capítulo anterior e mostrado no apêndice na Figura A-3. Neste exemplo numérico, uma análise de carga foi realizada com forças não correlacionadas sobre um tempo total de 500 seg. A configuração da carga vem dada pela localização das cargas nos 
graus de liberdade 5, 8 e 11 (graus de liberdade mostrados na Figura A-3). As forças têm uma densidade espectral de potência com uma frequência predominante de $30 \mathrm{~Hz}$. Um exemplo da excitação com sua associada densidade espectral são mostrados na Figura 5-1. A frequência de amostragem para excitação e a análise dinâmica foi de $350 \mathrm{~Hz}$. Os resultados das acelerações nos graus de liberdade $5,8,11,14$, e 17 foram usados para verificar o método de identificação.
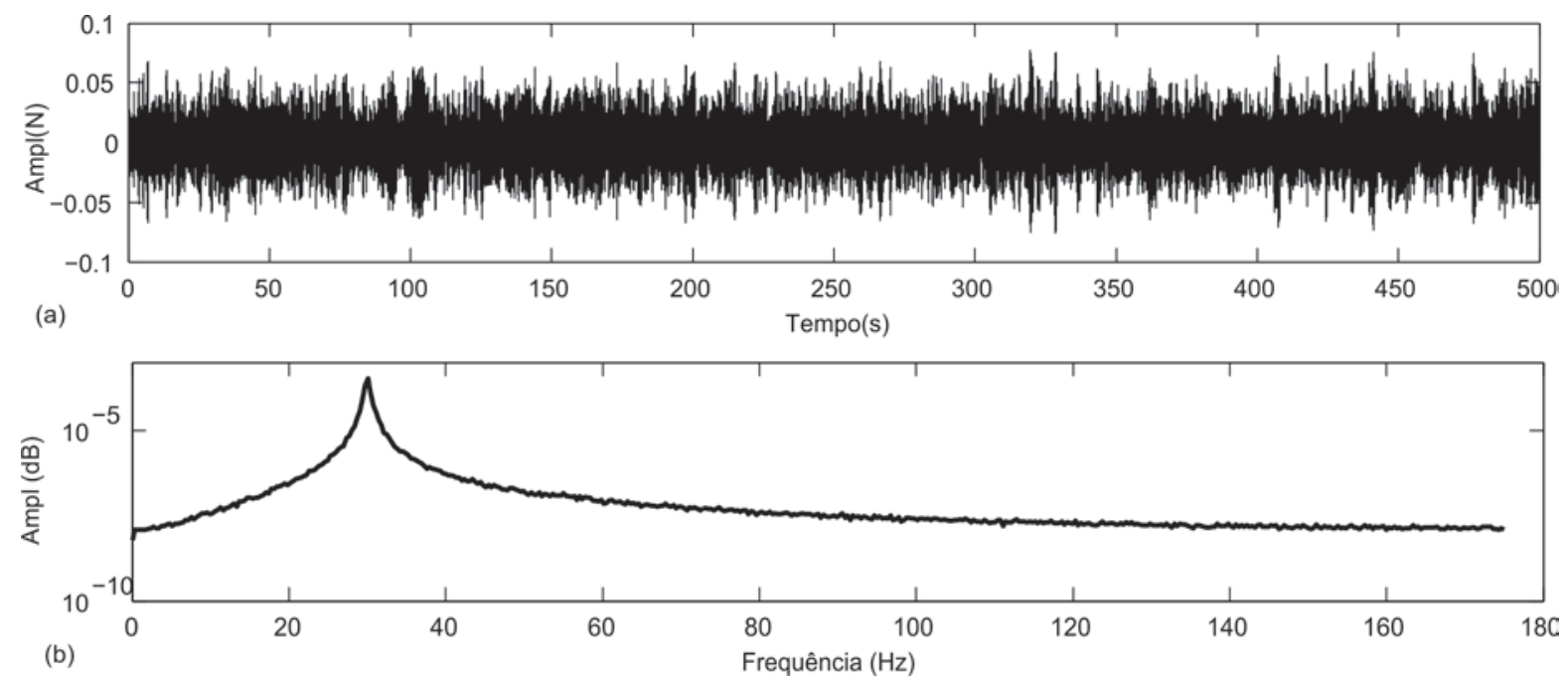

Figura 5-1 a) Exemplo da excitação, b) densidade espectral de potência da excitação usada no exemplo numérico.

Duas diferentes configurações para as coordenadas de $\mathrm{U}$ e $\mathrm{K}$ foram usadas para avaliar a matriz de transmissibilidade proposta na Equação (5-15) e identificar os mesmos parâmetros modais do sistema em cada uma delas. A primeira configuração foi definida como Set 1 e contém os graus de liberdade $U=5,8,11$ e $K=14,17$; a segunda configuração foi definida como Set 2 e contém os graus de liberdade $U=5,8,11,14,17$ e $K=17$. Dessa forma, 5 matrizes de transmissibilidade com diferentes referências da saída $Z_{i}$ foram definidas para cada das duas configurações de $U$ e $K$, em que as referências da saída $Z_{i}$ foram variadas entre os graus de liberdade 5,8,11,14 e 17. De acordo com as Equações (5-11) e (5-12), as matrizes de transmissibilidade multivariável com diferentes referências da saída para as duas configurações da saída U e K são dada por. 


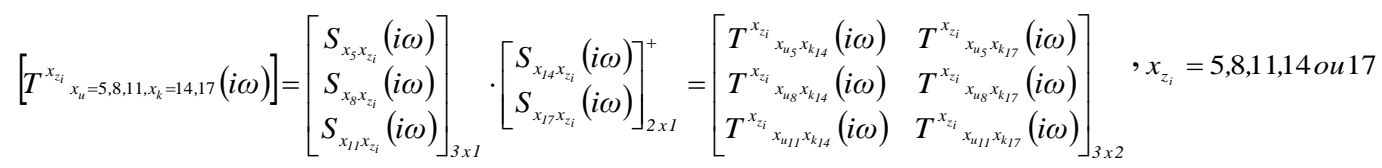

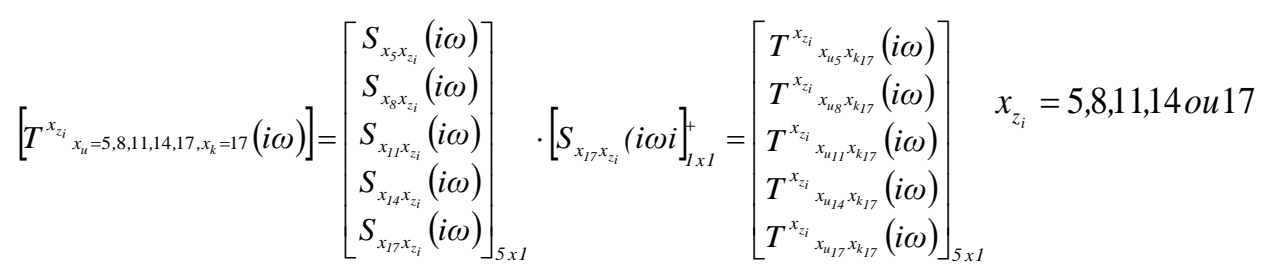

Nas Equações (5-21) e (5-22), as funções de densidade espectral de potência das matrizes $\left[S_{X_{u} x_{z}}(i \omega)\right]$ e $\left[S_{x_{k} x_{z}}(i \omega)\right]$ foram avaliadas usando o método de Welch com uma janela de Hanning do tamanho $2^{11}$, uma sobreposição do 50\% e um tamanho da transformada de Fourier de $2^{11}$. As matrizes de transmissibilidade multivariável mostradas nas Equações (5-21) e (5-22) foram usadas para construir a matriz proposta em acordo com a Equação (5-15). Portanto, dois matrizes surgem para cada uma das configurações das coordenadas de U e K (Set 1 e Set 2) e são dadas como.

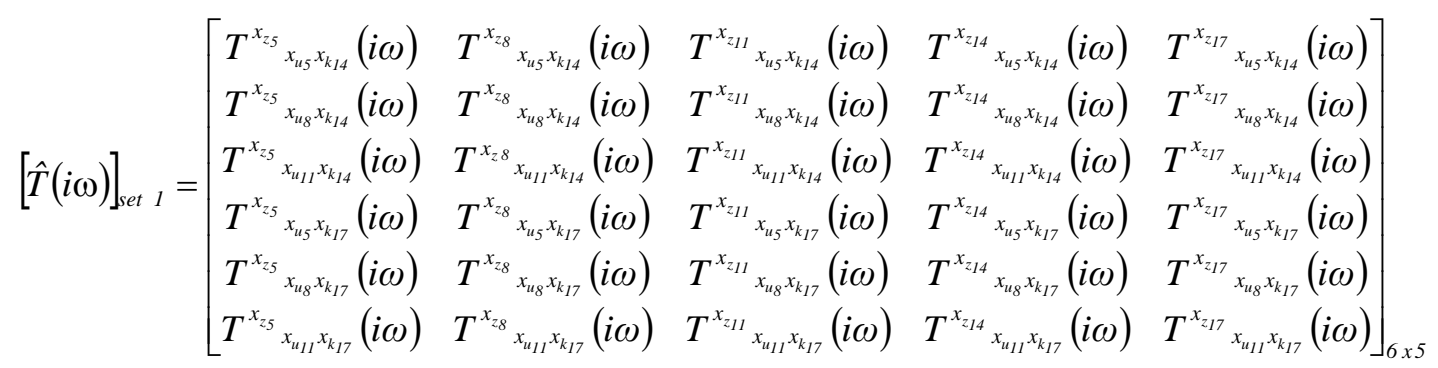

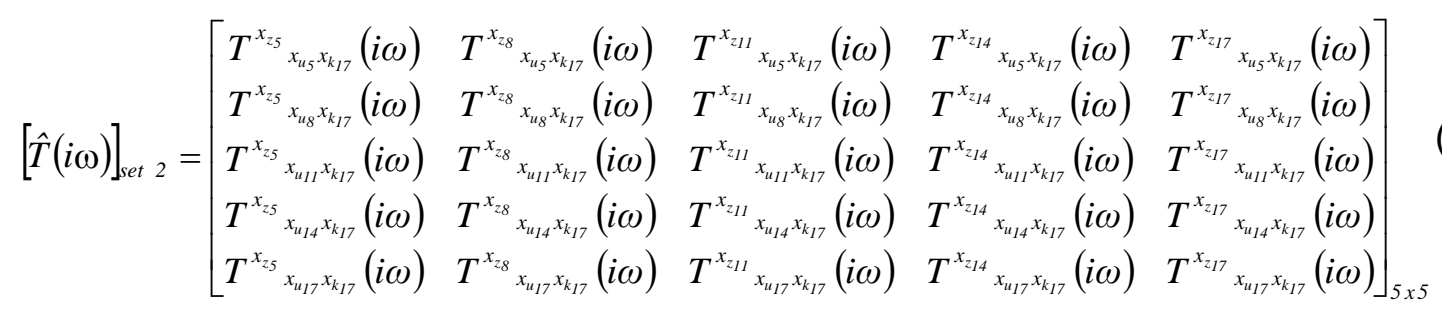

Ambas as matrizes nas Equações (5-23) e (5-24) foram usadas para estimar os parâmetros modais (frequências naturais amortecidas e taxas de amortecimento) em acordo com o procedimento descrito anteriormente. No entanto, para a estimação dos modos de vibração, uma condição deve ser satisfeita com respeito às coordenadas $U$ e $K$, a qual é obtida na segunda configuração das coordenadas K e U (Set 2). Portanto, está configuração foi usada no método proposto para estimar os diferentes modos de vibração do modelo do exemplo numérico.

Uma análise preliminar das frequências do modelo do exemplo numérico pode ser realizada calculando uma média normalizada das auto densidades espectrais a partir das respostas de aceleração do modelo, e analisar os picos de ressonância do espectro 
normalizado. Dessa forma, na Figura 5-2 mostram-se diferentes picos de ressonância na faixa de frequências de 0 até $175 \mathrm{~Hz}$. No entanto, os resultados dos picos na Figura 5-2 mostram uma frequência de ressonância de $30 \mathrm{~Hz}$ que não pertence ao modelo estrutural. Esse resultado é devido à excitação de ruído colorido usada com uma frequência predominante. O resultado também indica que neste exemplo qualquer método de identificação no domínio da frequência, como podem ser FDD (Brincker, Zhang e Andersen, 2000) ou PolyMAX (Peeters et al., 2004), os quais são aplicados diretamente sobre a matriz de densidade espectral de potência identificarão uma frequência adicional igual a $30 \mathrm{~Hz}$. Pelo contrário, a independência da excitação é uma importante vantagem do método proposto se comparado com os métodos tradicionais que consideram a excitação como ruído branco na análise modal de estruturas em operação.

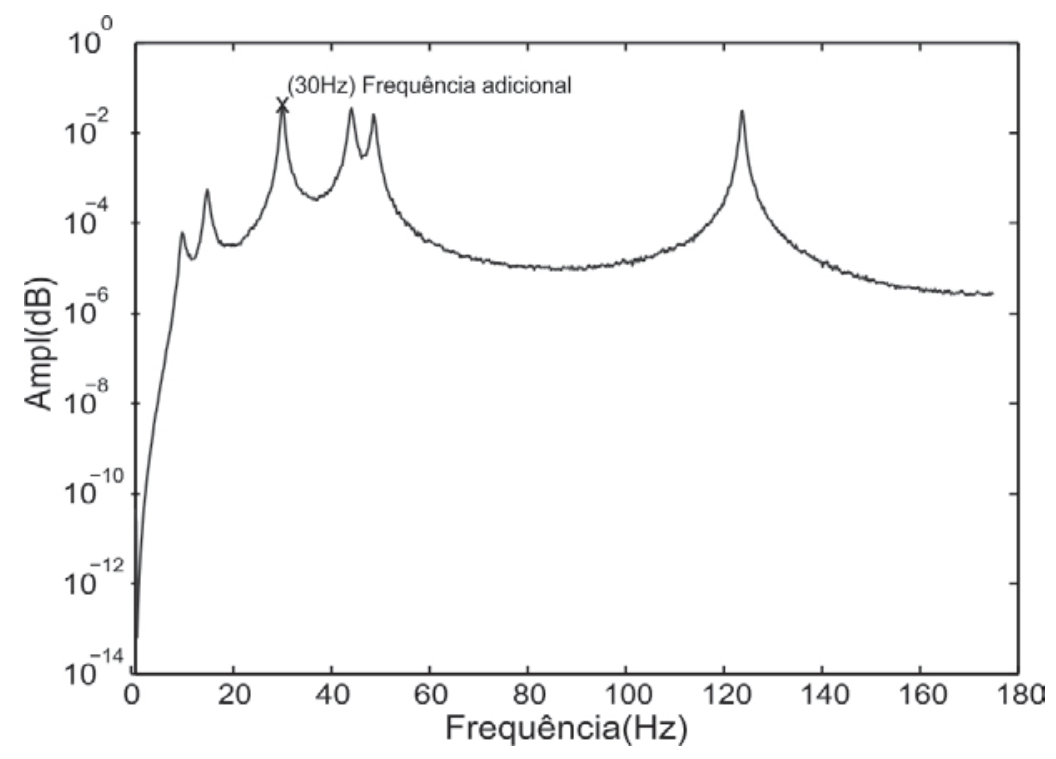

Figura 5-2 Média normalizada das auto-densidades espectrais.

No método proposto, o primeiro passo é aplicar a decomposição de valores singulares nas matrizes das Equações (5-23) (Set 1) e (5-24) (Set 2) e analisar a convergência para zero dos valores singulares a partir do segundo valor singular, como mostrado na Figura 5-3. Além disso, uma média de todos os valores na faixa de frequência de 0 até $175 \mathrm{~Hz}$ em cada valor singular é mostrada na Figura 5-3 . Na Figura 5-3 pode-se notar uma convergência para zero de diferentes frequências nos valores singulares para as configurações Set 1 e Set 2. 

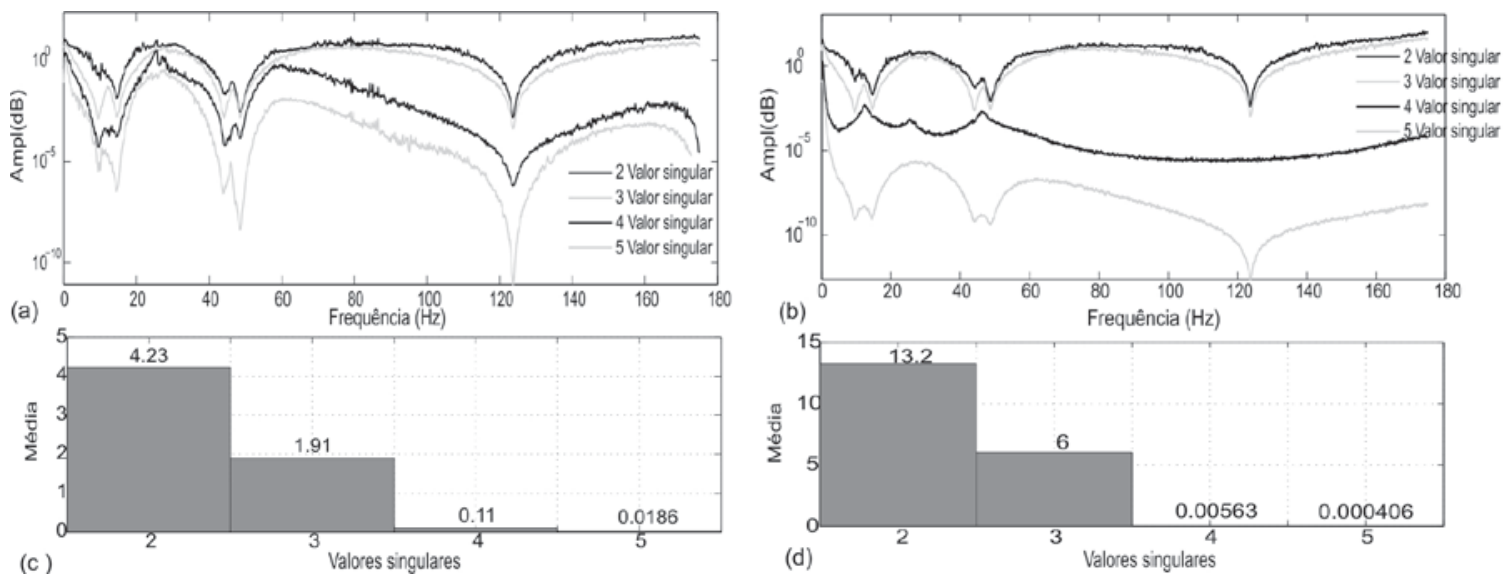

Figura 5-3 a),b) Decomposição de valores singulares e c), d) média de todos os valores na faixa de frequência de 0 até $175 \mathrm{~Hz}$ das matrizes definidas nas Equações (5-23) (Set 1) e (5-24) (Set 2), respectivamente.

Neste exemplo numérico, um número total de 3 cargas não correlacionadas foi usado, assim, o máximo posto das matrizes nas Equações (5-23) e (5-24) é igual a 3. Portanto, só os valores singulares 2 e 3 devem ser selecionados para calcular a matriz inversa, como mostrado na Equação (5-18). O critério proposto para a seleção do número de valores singulares, o qual é baseado em uma média para cada valor singular na faixa de frequência total, mostra que ambas as configurações Set1 e Set 2 têm os valores singulares aproximadamente iguais ao zero para os valores singulares 4 e 5 . Os valores singulares $4 \mathrm{e}$ 5 são considerados como ruído e não são usados para o cálculo da inversa em ambas as configurações Set 1 e Set 2. Este resultado mostra que o critério proposto funciona bem para o processo de tomada de decisão sobre o número de valores singulares que devem ser selecionados na inversa.

Os valores singulares 2 e 3 foram selecionados para avaliar as matrizes inversas das Equações (5-23) e (5-24) em acordo com a Equação (5-18). As funções das matrizes inversas $[\hat{T}(i \omega)]_{\text {set } 1}^{++}$e $[\hat{T}(i \omega)]_{\text {set } 2}^{++}$do tamanho $5 \times 6$ e $5 \times 5$, respectivamente, são mostradas na Figura 5-4. Na Figura 5-4 pode ser observado que para ambas as configurações Set 1 e Set 2, as frequências naturais do sistema são identificadas sem a introdução de frequências naturais adicionais produzidas pela excitação de ruído colorido usada. 

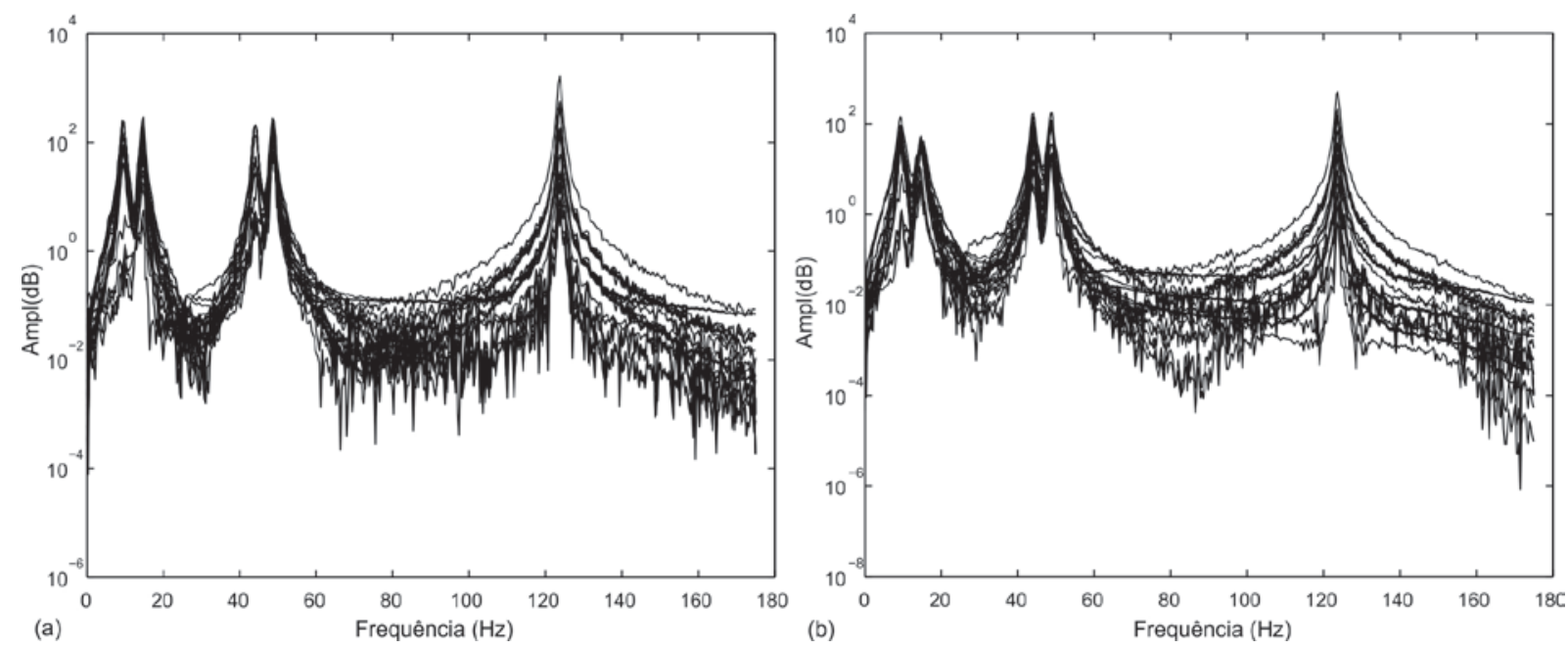

Figura 5-4 Funções das matrizes a) $[\hat{T}(i \omega)]_{\text {set1 }}^{++}$e b) $[\hat{T}(i \omega)]_{\text {set } 2}^{++}$.

O seguinte passo é aplicar o método PolyMAX nas matrizes $[\hat{T}(i \omega)]_{\text {set1 }}^{++}$e $[\hat{T}(i \omega)]_{\text {set } 2}^{++}$ para estimar as frequências naturais amortecidas e as taxas de amortecimento do sistema. O método PolyMAX pode ser usado em toda a matriz ou especificamente sobre colunas o linhas da matriz. Melhores resultados são obtidos se só é aplicado sobre uma linha ou uma coluna. Neste trabalho, o método PolyMAX foi usado sobre a primeira linhas das matrizes $[\hat{T}(i \omega)]_{s e t 1}^{++}$e $[\hat{T}(i \omega)]_{s e t 2}^{++}$. Nas Figuras 5-5 e 5-6 mostram-se os diagramas de estabilização construídos a partir da primeira linha das matrizes $[\hat{T}(i \omega)]_{s e t 1}^{++}$e $[\hat{T}(i \omega)]_{s e t 2}^{++}$, considerando uma ordem dos polinômios na faixa de 20 até 80 (ordem do modelo). O critério de estabilização para ambos os diagramas foi como segue: os pólos estáveis foram definidos como os pólos com um sinal negativo da parte real do pólo, e os pólos instáveis como aqueles como um sinal positivo na parte real do pólo. Portanto, nas Figura 5-5 e 5-6 os pólos estáveis são claramente distinguidos dos pólos instáveis somente pelo sinal da parte real do polo. Além disso, nas Figura 5-5 e 5-6 mostram-se as curvas de ajuste realizada pelo método PolyMAX com um ordem do modelo de 70 , as quais concordam com os dados das primeiras linhas das matrizes $[\hat{T}(i \omega)]_{\text {set } 1}^{++}$e $[\hat{T}(i \omega)]_{\text {set } 2}^{++}$. 

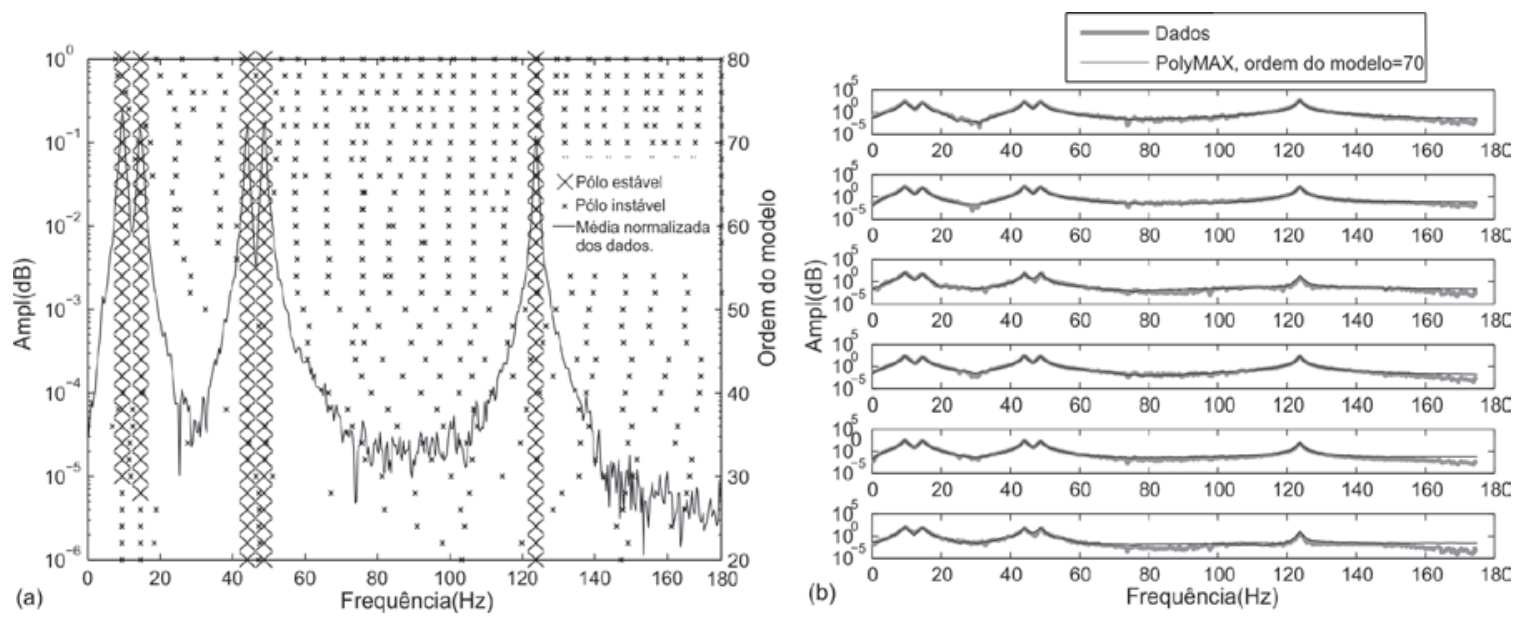

Figura 5-5 a) Diagrama de estabilidade e b) curva de ajuste usando o método PolyMAX aplicado na primeira linha da matriz $[\hat{T}(i \omega)]_{\text {set1 }}^{++}$.
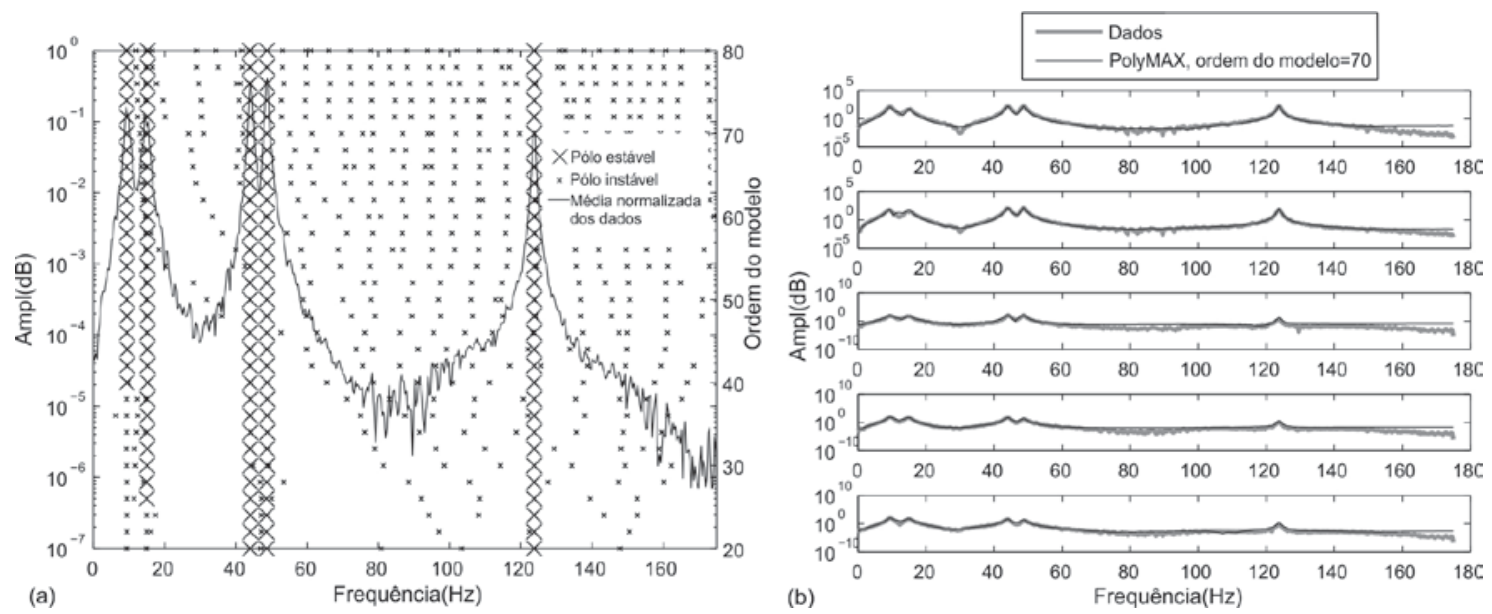

Figura 5-6 a) Diagrama de estabilidade e b) curva de ajuste usando o método PolyMAX aplicado na primeira linha da matriz $[\hat{T}(i \omega)]_{s e t 2}^{++}$.

Uma análise de estabilidade das taxas de amortecimento para cada um dos pólos estáveis mostrados nas Figura 5-5 e 5-6 foi usada para selecionar a ordem correta do modelo. A estabilidade das taxas de amortecimento foi alcançada para todos os pólos estáveis em uma ordem do modelo igual a 70 em ambos os diagramas de estabilidade, como mostrados na Figura 5-7. A Tabela 5-1 apresenta um resumo e comparação das frequências naturais amortecidas e as taxas de amortecimento identificadas para uma ordem do modelo de 70 com os valores teóricos do modelo em ambas as configurações Set 1 e Set 2. O resultado mostraram que para ambas as configurações Set 1 e Set 2 , os valores identificados se aproximam com os valores teóricos. 

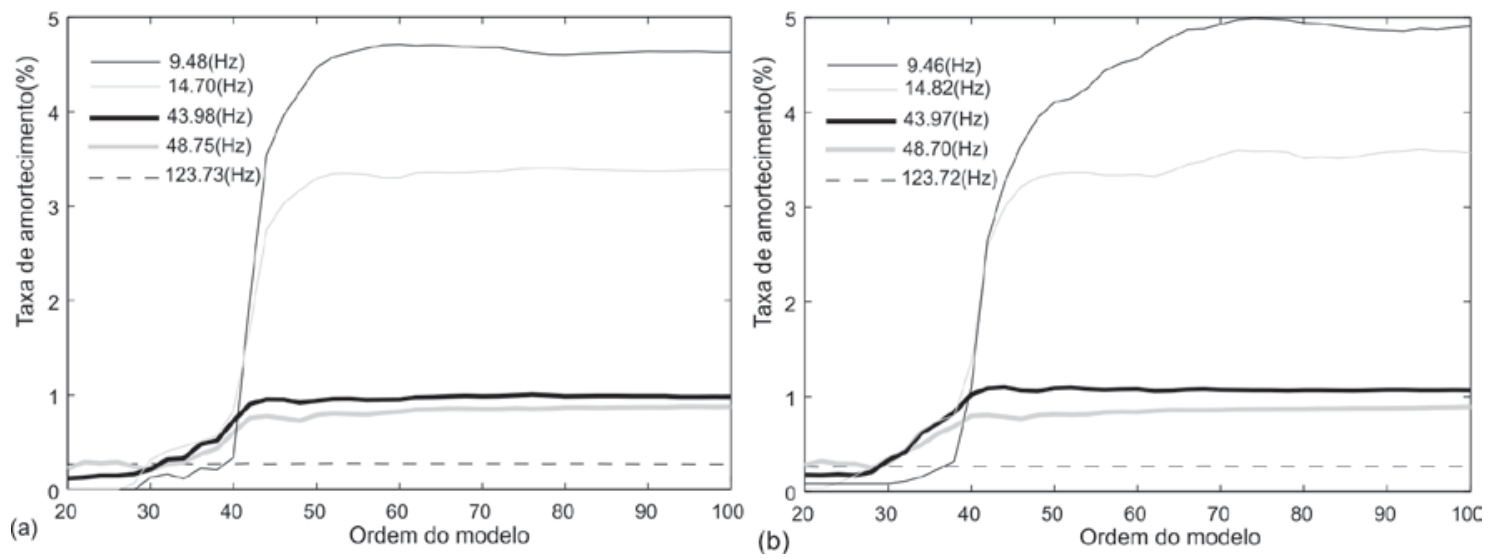

Figura 5-7 Estabilidade das taxas de amortecimento dos pólos estáveis extraídos da a) Figura 5-5 e b) Figura 5-6.

Tabela 5-1 Frequências naturais amortecidas e taxas de amortecimento identificadas usando o método proposto para ambas as configurações Set 1 e Set 2.

\begin{tabular}{|c|c|c|c|c|c|c|c|c|c|c|}
\hline \multirow[b]{3}{*}{ Modos } & \multirow{2}{*}{\multicolumn{2}{|c|}{ Valores teóricos }} & \multicolumn{4}{|c|}{ Estimados, ordem do modelo $=70$} & \multicolumn{4}{|c|}{ Erro (\%) } \\
\hline & & & \multicolumn{2}{|c|}{ Set 1} & \multicolumn{2}{|c|}{ Set 2} & \multicolumn{2}{|c|}{ Set 1} & \multicolumn{2}{|c|}{ Set 2} \\
\hline & $\begin{array}{l}\text { Frequências } \\
\text { naturais }(\mathrm{Hz})\end{array}$ & $\begin{array}{c}\text { Taxa } \\
\text { amortecim } \\
\text { ento }(\%)\end{array}$ & $\begin{array}{l}\text { Frequências } \\
\text { naturais }(\mathrm{Hz})\end{array}$ & $\begin{array}{c}\text { Taxa } \\
\text { amortecim } \\
\text { ento (\%) }\end{array}$ & $\begin{array}{l}\text { Frequências } \\
\text { naturais }(\mathrm{Hz})\end{array}$ & $\begin{array}{c}\text { Taxa } \\
\text { amortecim } \\
\text { ento (\%) }\end{array}$ & $\begin{array}{l}\text { Frequências } \\
\text { naturais }(\mathrm{Hz})\end{array}$ & $\begin{array}{c}\text { Taxa } \\
\text { amortecim } \\
\text { ento }(\%)\end{array}$ & $\begin{array}{l}\text { Frequências } \\
\text { naturais }(\mathrm{Hz})\end{array}$ & $\begin{array}{c}\text { Taxa } \\
\text { amortecim } \\
\text { ento (\%) }\end{array}$ \\
\hline 1 & 9.54 & 5.00 & 9.48 & 4.68 & 9.46 & 4.84 & 0.65 & 6.45 & 0.88 & 3.11 \\
\hline 2 & 14.62 & 3.27 & 14.70 & 3.37 & 14.82 & 3.55 & 0.61 & 2.92 & 1.40 & 8.60 \\
\hline 3 & 44.05 & 1.08 & 43.98 & 0.99 & 43.97 & 1.07 & 0.16 & 8.53 & 0.18 & 0.49 \\
\hline 4 & 48.70 & 0.98 & 48.75 & 0.85 & 48.70 & 0.86 & 0.10 & 13.10 & 0.01 & 12.31 \\
\hline 5 & 123.49 & 0.39 & 123.73 & 0.28 & 123.72 & 0.27 & 0.20 & 29.41 & 0.19 & 31.35 \\
\hline
\end{tabular}

Uma vez as frequências de ressonância são identificadas, o método proposto pode identificar os modos de vibração usando o primeiro vetor singular à esquerda $\hat{U}(i \omega)$ na frequência natural, $i \omega_{l}$. No entanto, os modos de vibração completos podem ser obtidos somente para a configuração Set 2 devido à condição requerida pela teoria. Portanto, os resultados da configuração Set 2 são mostrados e comparados com os valores teóricos a partir do critério de correlação modal (MAC) na Tabela 5-2 .Os modos de vibração identificados utilizando o método proposto e os valores teóricos como mostrados na Tabela 5-2 concordaram bem.

Tabela 5-2 Modos de vibração identificados usando o método proposto para Set 2.

\begin{tabular}{|c|c|c|c|c|c|c|c|c|c|c|}
\hline \multirow[b]{2}{*}{ Nodos } & \multicolumn{2}{|c|}{ Mode $1, \mathrm{MAC}=0.9988$} & \multicolumn{2}{|c|}{ Mode 2, MAC $=0.9999$} & \multicolumn{2}{|c|}{ Mode 3, MAC $=0.9991$} & \multicolumn{2}{|c|}{ Mode 4, MAC $=0.9980$} & \multicolumn{2}{|c|}{ Mode 5, MAC=1 } \\
\hline & Teóricos & Estimados & Teóricos & Estimados & Teóricos & Estimados & Teóricos & Estimados & Teóricos & Estimados \\
\hline 5 & 0.0315 & -0.5072 & -0.0361 & -0.5640 & -0.0376 & -0.5098 & -0.0325 & -0.4673 & 0.0068 & -0.0907 \\
\hline 8 & 0.0315 & -0.5139 & -0.0261 & -0.4050 & 0.0376 & 0.5121 & 0.0384 & 0.5472 & -0.0201 & 0.2665 \\
\hline 11 & 0.0000 & 0.0194 & -0.0019 & -0.0297 & 0.0000 & 0.0020 & 0.0120 & 0.1636 & 0.0695 & -0.9178 \\
\hline 14 & -0.0315 & 0.4830 & -0.0261 & -0.4137 & -0.0376 & -0.4809 & 0.0384 & 0.5029 & -0.0201 & 0.2652 \\
\hline 17 & -0.0316 & 0.4841 & -0.0361 & -0.5686 & 0.0376 & 0.4833 & -0.0325 & -0.4237 & 0.0068 & -0.0898 \\
\hline
\end{tabular}


A nova aproximação apresentada neste capítulo para a identificação de parâmetros modais tem demostrado as seguintes caraterísticas: a) uma independência das caraterísticas da excitação com a habilidade de identificar parâmetros modais sem a introdução de frequências adicionais produto da excitação, b) a extração de parâmetros modais usando uma única condição de carga e c) a identificação dos mesmos parâmetros modais a partir de gerar diferentes configurações das coordenadas $\mathrm{K}$ e $\mathrm{U}$. Esta última caraterística é particularmente atrativa para estabelecer uma confiabilidade e validade na identificação de parâmetros modais a partir de usar diferentes configurações de $\mathrm{K}$ e U.

No entanto, uma das variáveis importantes no método proposto para obter "bons" resultados na identificação de parâmetros modais é principalmente o número de cargas não correlacionadas que existem na estrutura. Por exemplo, se o exemplo numérico descrito acima possuía uma única carga de entrada, então, o posto máximo da matriz na Equação (5-15) seria 1, o que significaria o mesmo posto da matriz nos pólos do sistema. Assim, para está condição de carga, é impossível avaliar a singularidade da matriz na Equação (5-15) nos pólos do sistema e identificar os parâmetros modais. Teoricamente, o número mínimo das cargas de entrada em uma estrutura não deve ser menor do que 2 para avaliar os parâmetros modais usando o método proposto. O número de cargas em uma estrutura não deve ser um obstáculo na identificação modal de estrutura em operação, já que diferentes cargas (por exemplo, o vento, o trânsito, pedestre) são aplicadas simultaneamente em locais diferentes sobre a estrutura.

\subsection{DADOS REAIS DE VIBRAÇÃO}

Para avaliar o desempenho do método proposto, dados reais de vibração da ponte apresentada no Capítulo 4 foram usados. Nesta oportunidade dados de acelerações sobre o vão 6-7 da ponte foram utilizados e somente para a segunda configuração de acelerômetros (Setup 2), como se pode ver na Figura 4-8. Nesta aplicação sobre dados reais, as densidades espectrais de potência foram calculadas usando o método de Welch, o que conduz a uma matriz de densidades espectral cruzada do tamanho de $3 \times 3$. Os parâmetros para o método Welch foram definidos com uma janela do tamanho de $2^{11}$ com 50\% de sobreposição e um tamanho da transformada de Fourier de $2^{11}$.

Na Figura 5-8 mostram-se os resultados do método PolyMAX sendo aplicado diretamente sobre a primeira linha da matriz de densidade espectral (de aqui em adiante chamado como o método PolyMAX-PSD). Aplicando o método PolyMAX foram encontradas seis linhas com pólos estáveis na faixa de frequência de 0 até $20 \mathrm{~Hz}$, as quais correspondem com os primeiros modos de vibração da ponte. Na Figura 5-8 também é mostrada a estabilidade das taxas de amortecimento para cada pólo estável. É importante deixar claro 
que o método PolyMAX foi aplicado à faixa de frequência de $0-100 \mathrm{~Hz}$, no entanto para simplificar a quantidade de informação apenas os resultados da faixa de frequências de 0$20 \mathrm{~Hz}$ são mostrados nas figuras Figura 5-8,5-10 e 5-11.
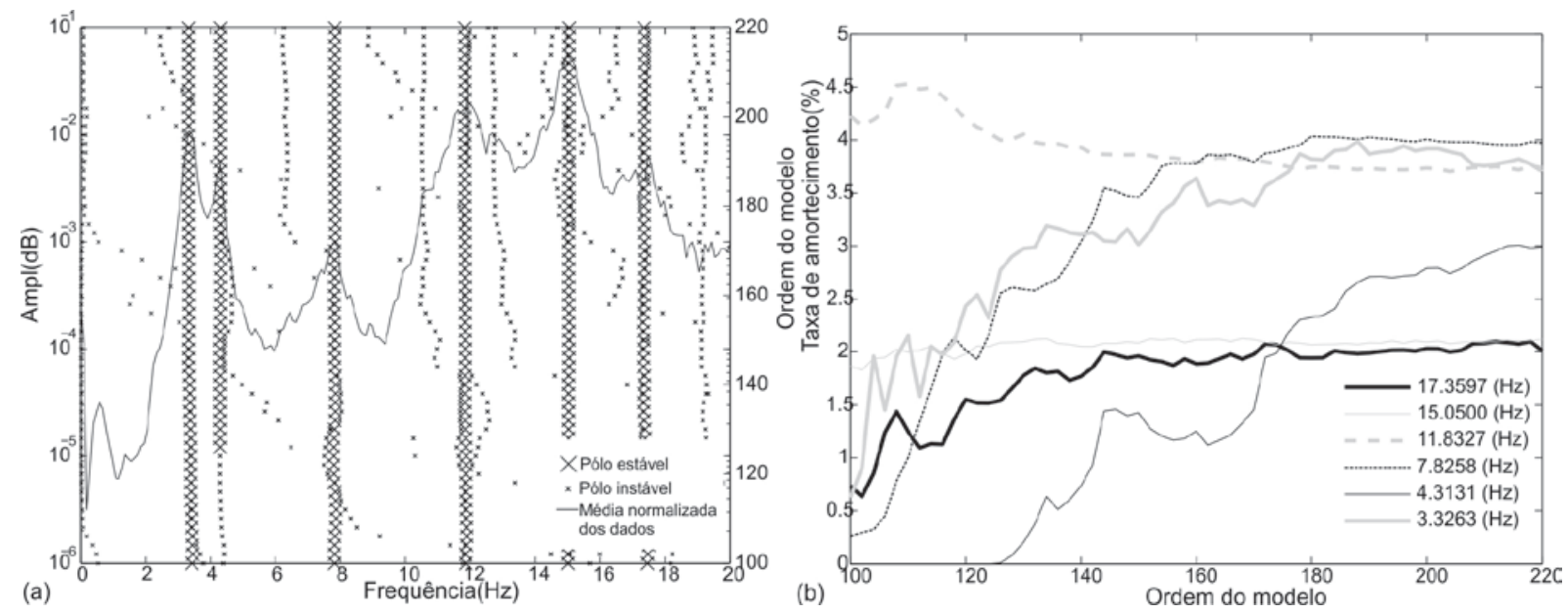

Figura 5-8 a) diagrama de estabilidade e b) estabilidade das taxas de amortecimento para os pólos estáveis determinados a partir do método PolyMAX aplicado na matriz de densidade espectral.

No método proposto foram definidas duas configurações diferentes para as coordenadas $\mathrm{K}$ e U. A primeira delas é dada por Set A com a localização dos acelerômetros para $U=a 1, a 2$ e $K=a 3$. A segunda é dada por Set $B$ com a localização dos acelerômetros para $\mathrm{U}=\mathrm{a1}, \mathrm{a} 2, \mathrm{a} 3$ e $\mathrm{K}=\mathrm{a} 3$. A partir destas configurações e usando a Equação (5-15), duas diferentes matrizes $2 \times 3$ e $3 \times 3$ podem ser definidas para Set $A$ e Set $B$, respectivamente. Uma decomposição de valores singulares foi aplicada nas matrizes definidas por Set $A$ e Set $B$ para calcular uma média para cada valor singular na faixa de frequências de 0 até $100 \mathrm{~Hz}$, como é mostrado na Figura 5-9. A Figura 5-9 mostra a média dos valores singulares e uma convergência para zero de diferentes frequências que correspondem com as frequências naturais. A média ajuda a determinar o número de valores singulares que podem ser selecionados no cálculo da matriz inversa. Por conseguinte, no cálculo da matriz inversa para ambas as matrizes $[\hat{T}(i \omega)]_{\text {set A }}^{++}$e $[\hat{T}(i \omega)]_{\text {setB }}^{++}$somente o segundo valor singular foi selecionado, removendo o terceiro valor singular da configuração Set B. 

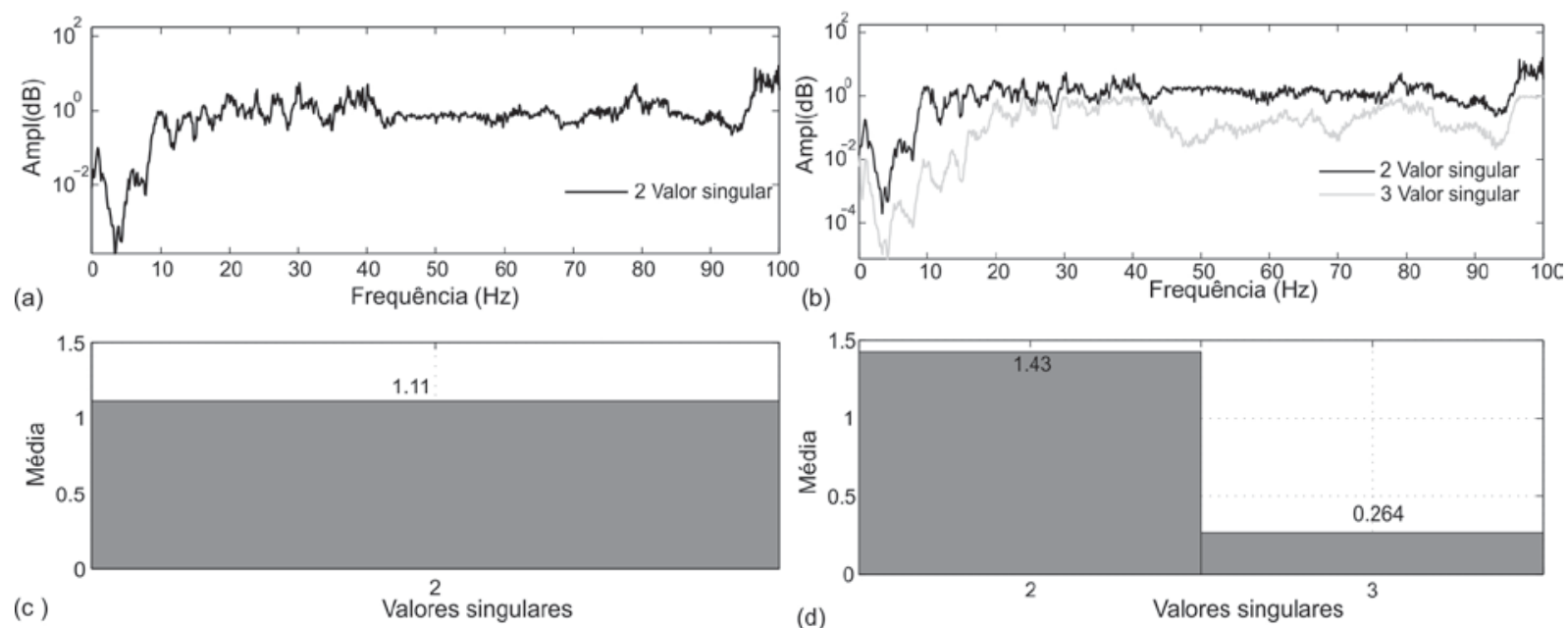

Figura 5-9 a), b) Decomposição de valores singulares e c), d) média dos valores singulares na faixa de frequência de 0 até $100 \mathrm{~Hz}$ para as matrizes de Set $\mathrm{A}$ e Set $\mathrm{B}$, respectivamente.

O método PolyMAX foi aplicado sobre a primeira linha das matrizes $[\hat{T}(i \omega)]_{s e t ~ A}^{++}$e $[\hat{T}(i \omega)]_{\text {setB }}^{++}$para estimar as frequências naturais amortecidas e as taxas de amortecimento da ponte. As Figuras 5-10 e 5-11 mostram os diagramas de estabilidade dos pólos estáveis e a estabilidade das taxas de amortecimento na faixa de frequências de 0 até $20 \mathrm{~Hz}$. As Figuras 5-10 e 5-11 mostram que os mesmos pólos estáveis foram encontrados para ambas as configurações Set A e Set B em comparação com o método PolyMAX-PSD. Este acordo entre o método PolyMAX-PSD e o método proposto verifica que não existem frequências predominantes produto da excitação devido a que o método proposto não depende da excitação ao contrário do método PolyMAX-PSD. As frequências e as taxas de amortecimento identificadas a partir do método PolyMAX-PSD e o método proposto são resumidas na Tabela 5-3. Em geral, os resultados mostram que o método PolyMAX-PSD e o método proposto concordam na identificação das frequências naturais. No entanto, maiores diferenças foram encontradas na identificação das taxas de amortecimento entre os dois métodos, mas valores similares de amortecimento foram encontrados para as duas configurações Set A e Set B no método proposto. 

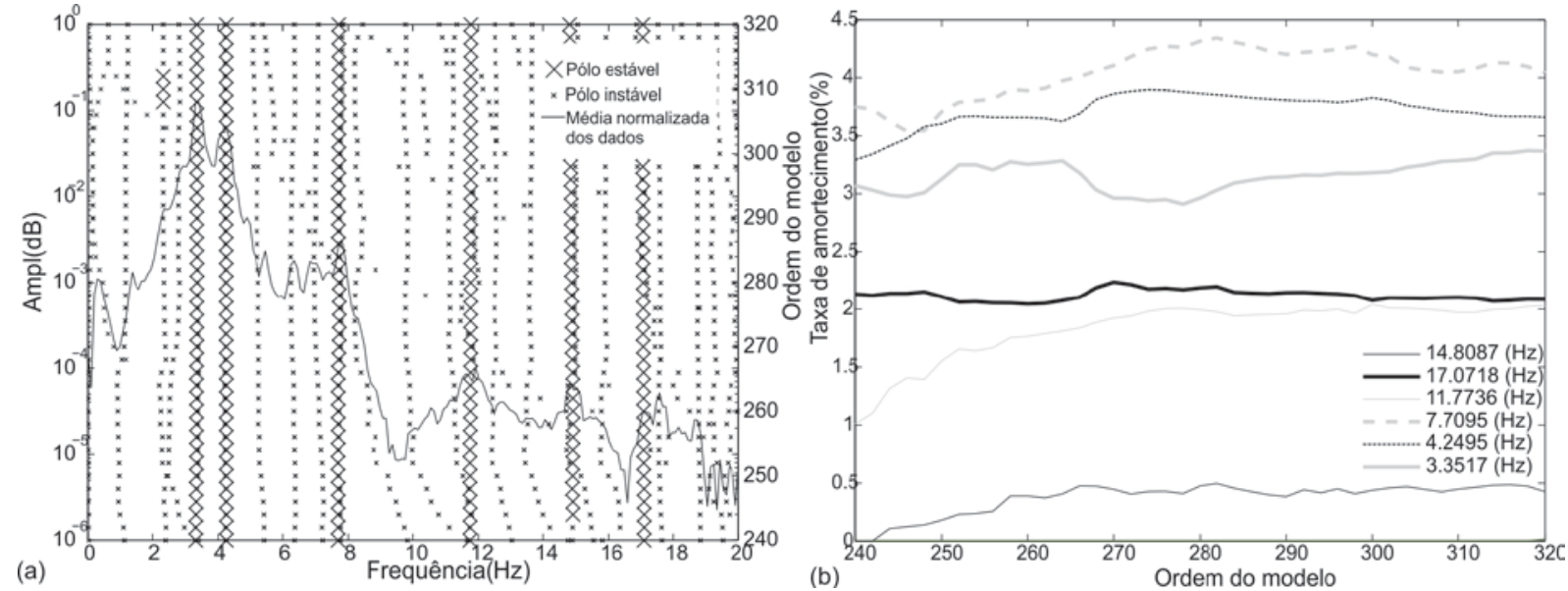

Figura 5-10 a) diagrama de estabilidade e b) estabilidade das taxas de amortecimento para os pólos estáveis determinados usando o método PolyMAX na matriz inversa $[\hat{T}(i \omega)]_{\text {set A }}^{++}$.
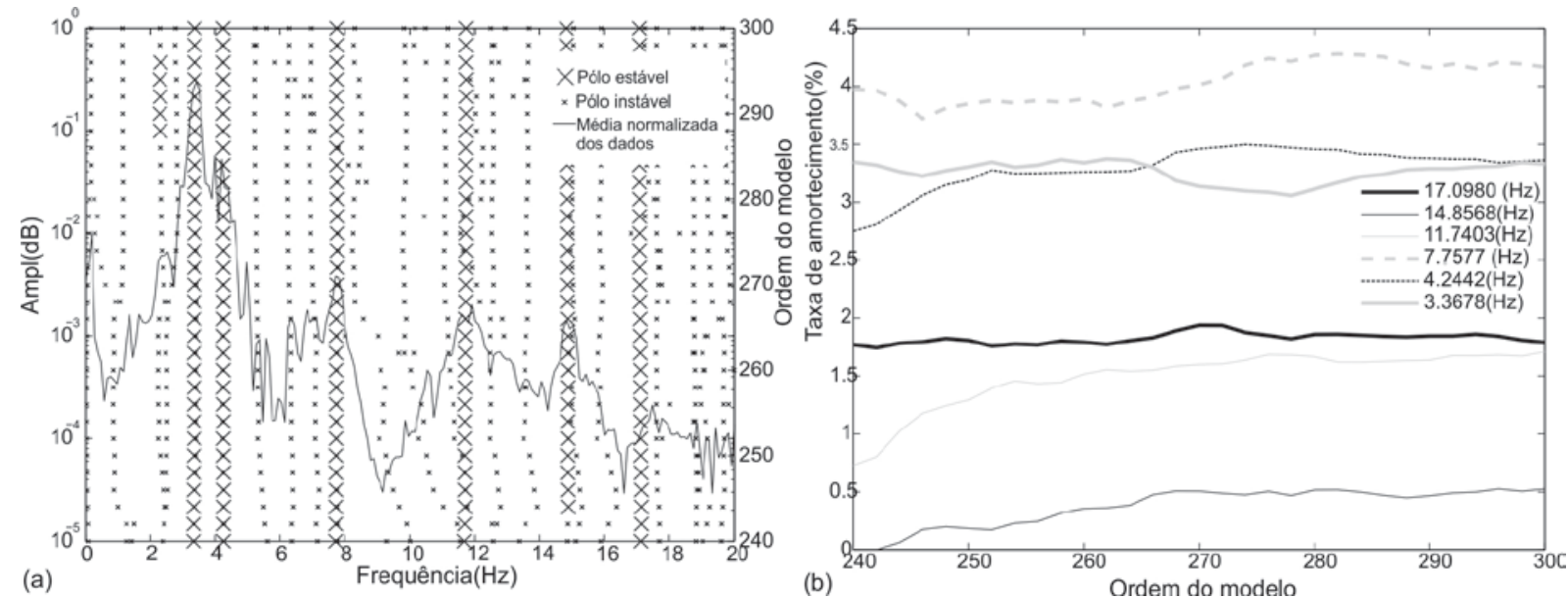

Figura 5-11 a) diagrama de estabilidade e b) estabilidade das taxas de amortecimento para os pólos estáveis determinados usando o método PolyMAX na matriz inversa $[\hat{T}(i \omega)]_{\text {setB }}^{++}$ 
Tabela 5-3 Frequências naturais e taxas de amortecimento identificadas da ponte a partir do método proposto e o PolyMAX-PSD

\begin{tabular}{|c|c|c|c|c|c|c|}
\hline \multirow[b]{2}{*}{ Modos } & \multicolumn{2}{|c|}{$\begin{array}{c}\text { PolyMAX-PSD } \\
\text { ordem do modelo } 220 \\
\end{array}$} & \multicolumn{2}{|c|}{$\begin{array}{l}\text { Método proposto (Set A) } \\
\text { ordem do modelo } 320\end{array}$} & \multicolumn{2}{|c|}{$\begin{array}{c}\text { Método proposto (Set } \mathrm{A} \text { ) } \\
\text { ordem do modelo } 300\end{array}$} \\
\hline & $\begin{array}{c}\text { Frequências } \\
\text { amortecida }(\mathrm{Hz})\end{array}$ & $\begin{array}{c}\text { Taxa } \\
\text { amortecim } \\
\text { ento(\%) }\end{array}$ & $\begin{array}{c}\text { Frequências } \\
\text { amortecida }(\mathrm{Hz})\end{array}$ & $\begin{array}{c}\text { Taxa } \\
\text { amortecim } \\
\text { ento(\%) }\end{array}$ & $\begin{array}{c}\text { Frequências } \\
\text { amortecida }(\mathrm{Hz})\end{array}$ & $\begin{array}{c}\text { Taxa } \\
\text { amortecime } \\
\text { nto(\%) }\end{array}$ \\
\hline 1 & 3.3263 & 3.7036 & 3.3517 & 3.3673 & 3.3678 & 3.1140 \\
\hline 2 & 4.3131 & 2.9926 & 4.2495 & 3.6597 & 4.2442 & 3.4560 \\
\hline 3 & 7.8258 & 3.9726 & 7.7095 & 4.0427 & 7.7577 & 4.2684 \\
\hline 4 & 11.8327 & 3.7377 & 11.7736 & 2.0319 & 11.7403 & 1.6662 \\
\hline 5 & 15.0500 & 2.1174 & 14.8087 & 0.4262 & 14.8568 & 0.5152 \\
\hline 6 & 17.3597 & 2.0105 & 17.0718 & 2.0869 & 17.0980 & 1.8588 \\
\hline
\end{tabular}

Os modos de vibração foram estimados usando o método proposto com o primeiro vetor singular à esquerda a partir da matriz $\hat{\mathbf{T}}(\mathbf{i} \omega)_{\text {setB }}^{++}$nas frequências naturais identificadas. Os modos de vibração identificados a partir do método PolyMAX-PSD e o método proposto foram comparados com o MAC, como mostrado na Tabela 5-4. Os resultados mostram que ambos os métodos concordam bem.

Tabela 5-4 Modos de vibração identificados da ponte a partir do método proposto e o PolyMAX-PSD.

\begin{tabular}{|c|c|c|c|c|c|c|c|c|c|c|c|}
\hline \multicolumn{2}{|c|}{ Modo $1, \mathrm{MAC}=0,998$} & \multicolumn{2}{|c|}{ Modo $2, \mathrm{MAC}=0,994$} & \multicolumn{2}{|c|}{ Modo $3, \mathrm{MAC}=0,993$} & \multicolumn{2}{|c|}{ Modo $4, \mathrm{MAC}=0,998$} & \multicolumn{2}{|c|}{ Modo 5, MAC $=0,996$} & \multicolumn{2}{|c|}{ Modo $6, \mathrm{MAC}=0,982$} \\
\hline $\begin{array}{l}\text { PolyMAX- } \\
\text { PSD }\end{array}$ & $\begin{array}{l}\text { Método } \\
\text { proposto }\end{array}$ & $\begin{array}{l}\text { PolyMAX- } \\
\text { PSD }\end{array}$ & $\begin{array}{l}\text { Método } \\
\text { proposto }\end{array}$ & $\begin{array}{l}\text { PolyMAX- } \\
\text { PSD }\end{array}$ & $\begin{array}{l}\text { Método } \\
\text { proposto }\end{array}$ & $\begin{array}{l}\text { PolyMAX- } \\
\text { PSD }\end{array}$ & $\begin{array}{l}\text { Método } \\
\text { proposto }\end{array}$ & $\begin{array}{l}\text { PolyMAX- } \\
\text { PSD }\end{array}$ & $\begin{array}{l}\text { Método } \\
\text { proposto }\end{array}$ & $\begin{array}{l}\text { PolyMAX- } \\
\text { PSD }\end{array}$ & $\begin{array}{l}\text { Método } \\
\text { proposto }\end{array}$ \\
\hline 0.539 & 0.610 & -0.721 & -0.605 & -0.683 & -0.557 & 1.000 & 1.000 & -1.186 & -1.076 & 0.693 & 0.596 \\
\hline 1.000 & 1.000 & -1.000 & -1.000 & -1.000 & -1.000 & 0.162 & 0.169 & -0.145 & -0.190 & 1.000 & 1.000 \\
\hline 0.682 & 0.684 & -0.672 & -0.685 & -0.672 & -0.668 & -1.039 & -0.956 & 1.000 & 1.000 & 0.625 & 0.433 \\
\hline
\end{tabular}

Esta aplicação sobre dados reais de vibração de uma ponte e sua comparação com o método PolyMAX-PSD demonstraram que o método proposto pode identificar os mesmos parâmetros modais de uma estrutura através de diferentes configurações para as coordenadas $\mathrm{K} \mathrm{e} \mathrm{U}$. Esta constatação foi observada nos resultados das frequências naturais amortecidas e as taxas de amortecimento obtidas para as configurações Set A e Set B.

\subsection{CONSIDERAÇÕES FINAIS}

No presente capítulo foi proposto o conceito de funções de transmissibilidade multivariável com diferentes referências da saída, a qual é uma extensão do escalar PSDT. O multivariável PSDT proposto pode relacionar múltiplas saídas ao invés de unicamente duas. Diferentes multivariável PSDT com diferentes referências da saída convergem na mesma matriz nos pólos do sistema. Esta propriedade foi usada para prover uma nova alternativa na identificação de parâmetros modais. Similar ao escalar PSDT a multivariável PSDT não depende das características da excitação e os parâmetros modais podem ser 
extraídos usando uma única condição de carga. No entanto, o uso da multivariável PSDT para identificar parâmetros modais provê a possibilidade de extrair os mesmos parâmetros modais pela variação de diferentes relações da saída. Esta característica proporciona uma confiabilidade e a validação na identificação de parâmetros modais pela avaliação de diferentes relações entre as saídas. 



\section{CAPÍTULO 6. MÉTODO PSDTM-SVD MELHORADO.}

No Capítulo 4 foi proposto o uso de matrizes de transmissibilidade com densidade espectral para a identificação de parâmetros modais a partir do uso do conceito da transmissibilidade escalar com densidade espectral (PSDT). O método de identificação modal chamado PSDTM-SVD consiste basicamente em aplicar a técnica matemática SVD nas matrizes construídas a partir das PSDTs (Araújo e Laier, 2014). As diferentes matrizes com PSDTs convergem nas colunas à mesma relação de amplitudes de modos de vibração nos pólos do sistema, o que significa que elas são linearmente dependente ou de posto 1 nesses pólos. Esta propriedade de dependência linear nas matrizes de transmissibilidade nos pólos do sistema gera as seguintes caraterísticas: 1) os valores singulares a partir do segundo valor singular convergem para zero nos pólos do sistema e 2) o primeiro vetor singular à esquerda converge para os modos de vibração. Dessa forma, a partir do uso de valores e vetores singulares aplicando a técnica SVD nas matrizes de transmissibilidade, os parâmetros modais como frequências naturais e modos de vibração podem ser determinados.

No entanto, o método PSDTM-SVD como é proposto no Capítulo 4 é desprovido da habilidade para a identificação de taxas de amortecimento. Uma alteração no amortecimento modal na função resultante do método PSDTM-SVD foi gerada pelas operações matemáticas propostas entre os valores singulares para a identificação das frequências naturais (ver Equação (4-8)). Devido a isso, um tratamento matemático mais adequado que não gere alteração no amortecimento modal deve ser aplicado nas matrizes de transmissibilidade do método PSDTM-SVD. Nesse sentido, no Capítulo 5 a inversa MoorePenrose foi utilizado como ferramenta matemática para a identificação de parâmetros modais, e dentro desses parâmetros as taxas de amortecimentos modais (Devriendt et al. (2013)). Porém, uma modificação sobre a inversa foi proposta, a qual consistiu na eliminação da contribuição do primeiro valor singular, e o uso somente dos primeiros $k$ valores singulares. O número total de $k$ valores singulares que deve ser utilizado na inversa é diretamente definido pelo número de cargas não correlacionadas existentes na estrutura.

Desta forma, baseados na limitação comentada do método PSDTM-SVD é proposto neste capítulo uma melhora do método PSDTM-SVD. O método PSDTM-SVD melhorado consiste no uso das mesmas matrizes de transmissibilidade propostas no Capítulo 4, mas aplicando sobre elas uma inversa truncada Moore-Penrose como ferramenta matemática 
para a identificação de parâmetros modais. Depois de aplicar a inversa truncada em cada uma das matrizes de transmissibilidade uma redução da informação das matrizes inversas é realizada a partir dos primeiros valores singulares de cada matriz inversa. Assim, uma função resultante final do método é proposta através de uma média ponderada entre os primeiros valores singulares de cada matriz inversa. Um procedimento de identificação automática do número de valores singulares a serem empregadas na inversa é também proposto neste método aprimorando o procedimento dado no Capítulo 5 que depende da decisão do usuário. Finalmente, uma análise numérica foi realizada para avaliar o desempenho do método proposto.

\subsection{IDENTIFICAÇÃO DE PARÂMETROS MODAIS USANDO O MÉTODO PSDTM-SVD MELHORADO}

No método PSDTM-SVD é definida uma matriz de transmissibilidade a partir de diferentes funções de transmissibilidade escalares com densidade espectral representada da seguinte forma.

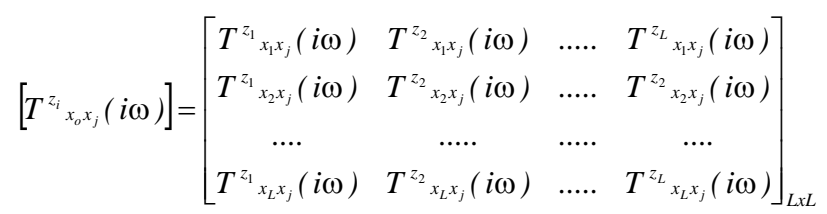

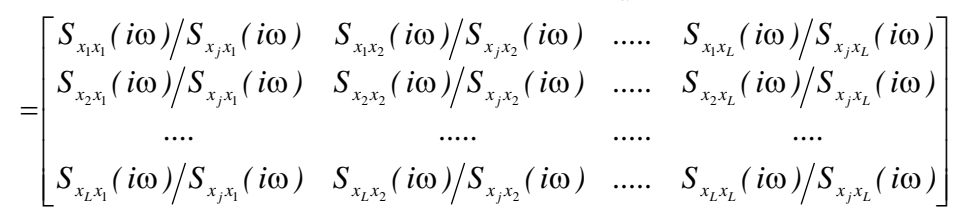

Em que, L é o número total de pontos de medição.

Dessa forma, diferentes matrizes de transmissibilidade de tamanho $L x L$ podem surgir a traves de uma variação do índice $\mathbf{j}$ nos $\mathrm{L}$ pontos de medição, $\left.\left.\mid T^{z_{i}}{ }_{x_{0} x_{1}}(i \omega)\right], \quad\left[T^{z_{i}}{ }_{x_{0} x_{2}}(i \omega)\right], \ldots \mid T^{z_{i}}{ }_{x_{0} x_{L}}(i \omega)\right]$. Igualmente, foi mostrado que cada matriz de transmissibilidade converge à seguinte relação quando se aproxima nos pólos do sistema.

$$
\lim _{i \omega \rightarrow i \omega_{l}}\left[T^{z_{i}}{ }_{{ }_{o} x_{j}}(i \omega)\right]=\left[\begin{array}{cccc}
\frac{\phi^{*}{ }_{1 l}}{\phi^{*}{ }_{j l}} & \frac{\phi^{*}{ }_{1 l}}{\phi_{j l}} & \ldots & \frac{\phi^{*}{ }_{1 l}}{\phi^{*}{ }_{j l}} \\
\frac{\phi^{*}{ }_{2 l}}{\phi^{*}{ }_{j l}} & \frac{\phi^{*}{ }_{2 l}}{\phi^{*}{ }_{j l}} & \ldots & \frac{\phi^{*}{ }_{2 l}}{\phi^{*}{ }_{j l}} \\
\ldots \ldots & \ldots \ldots & \ldots & \ldots . \\
\frac{\phi^{*}{ }_{L l}}{\phi^{*}{ }_{j l}} & \frac{\phi^{*}{ }_{L l}}{\phi^{*}{ }_{j l}} & \ldots & \frac{\phi^{*}}{\phi^{*}{ }_{j l}}
\end{array}\right]
$$

$$
\mathrm{Na} \quad \text { Equação (6-2) }
$$

mostra que

cada

matriz $\left[T^{z_{i}}{ }_{x_{0} x_{1}}(i \omega)\right], \quad\left[T^{z_{i}}{ }_{x_{0} x_{2}}(i \omega)\right], \ldots \quad\left[T^{z_{i}} x_{o} x_{L}(i \omega)\right]$ é linearmente dependente ou possui posto igual 
a 1 quando se aproxima nos pólos do sistema. Portanto, cada matriz

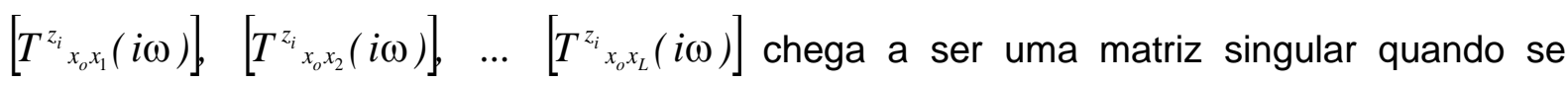
aproxima nos pólos do sistema. A singularidade de cada matriz pode ser avaliada usando a inversa generalizada Moore-Penrose com as modificações propostas no Capítulo 5. Essas modificações consistem em: eliminar a contribuição do primeiro valor singular e aplicar uma somatória truncada usando somente os primeiros $k$ valores singulares. Com essas modificações a inversa de cada matriz $\left.\left.\left.\mid T^{z_{i}}{ }_{x_{o} x_{1}}(i \omega)\right], \quad \mid T^{z_{i}}{ }_{x_{o} x_{2}}(i \omega)\right], \ldots \mid T^{z_{i}}{ }_{x_{o} x_{L}}(i \omega)\right\rfloor$ pode ser definida como.

$$
\begin{aligned}
{\left[T_{x_{o} x_{j}}^{z_{i}}(i \omega)\right]^{++} } & =\sum_{i=2}^{k} \frac{1}{\sigma_{i}(i \omega)}\left\{\hat{V}(i \omega)_{i}\right\} \cdot\left\{\hat{U}(i \omega)_{i}\right\}^{T} \\
& =\left[T_{x_{o} x_{j}}^{z}(i \omega)\right]_{2}^{+}+\left[T_{x_{o} x_{j}}^{z}(i \omega)\right]_{3}^{+}+\ldots+\left[T_{x_{o} x_{j}}^{z}(i \omega)\right]_{k}^{+}
\end{aligned}
$$

Em que, $\mathrm{k}$ deve ser menor ou igual ao número de cargas não correlacionadas presentes na estrutura. Da mesma maneira, por causa da singularidade nos pólos do

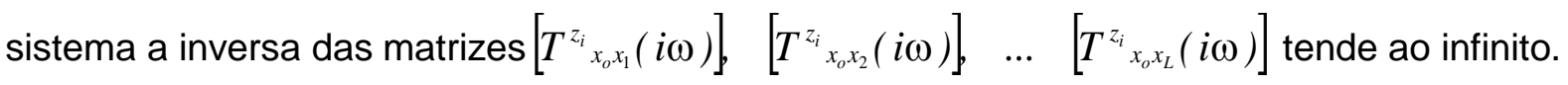

$\lim _{i \omega \rightarrow i \omega_{l}}\left[T^{z_{i}}{ }_{x_{o} x_{j}}(i \omega)\right]^{++}=\left[\begin{array}{cccc}\infty & \infty & \ldots . . & \infty \\ \infty & \infty & \ldots . . & \infty \\ \ldots & \ldots . . & \ldots . . & \ldots . . \\ \infty & \infty & \ldots . . & \infty\end{array}\right]_{L \times L}$

Em vista disso, os parâmetros modais podem ser identificados em cada uma das funções destas matrizes inversas. Consequentemente, assim como no Capítulo 5, o método PolyMAX poderia ser usado para modelar as matrizes $\left[T^{z_{i}}{ }_{x_{0} x_{1}}(i \omega)\right]^{++},\left[T^{z_{i}}{ }_{x_{0} x_{2}}(i \omega)\right]^{++}, \ldots\left[T^{z_{i} x_{o} x_{L}}(i \omega)\right]^{++}$e estimar as frequências de ressonância amortecidas e as taxas de amortecimentos. No entanto, é importante mencionar que cada uma das matrizes $\left[T^{z_{i}}{ }_{x_{0} x_{1}}(i \omega)\right]^{++},\left[T^{z_{i}}{ }_{x_{0} x_{2}}(i \omega)\right]^{++}, \ldots\left[T^{z_{i}}{ }_{x_{0} x_{L}}(i \omega)\right]^{++}$não contém informação de modos de vibração. Elas somente contêm informação de frequências de ressonância e das taxas de amortecimento. Portanto, o método PolyMAX não pode identificar modos de vibração a partir das matrizes $\left[T^{z_{i}}{ }_{x_{0} x_{1}}(i \omega)\right]^{++},\left[T^{z_{i}}{ }_{x_{o} x_{2}}(i \omega)\right]^{++}, \ldots\left[T^{z_{i}}{ }_{x_{o} x_{L}}(i \omega)\right]^{++}$. Geralmente, o método PolyMAX é aplicado a uma coluna ou linha da matriz de dados de entrada com o objetivo de identificar os modos de vibração. Nesse caso, em que somente podem ser avaliados frequências naturais e taxas de amortecimento, uma aplicação direta do método 
PolyMAX a só uma função ou elemento das matrizes $\left[T^{z_{i}}{ }_{x_{0} x_{1}}(i \omega)\right]^{++},\left[T^{z_{i}}{ }_{x_{0} x_{2}}(i \omega)\right]^{++}, \ldots\left[T^{z_{i}} x_{o} x_{L}(i \omega)\right]^{++}$pode ser empregada para identificar os parâmetros modais de interesse. Neste sentido, o método PSTDM-SVD melhorado propõe aplicar uma decomposição de valores singulares nas matrizes $\left[T^{z_{i}}{ }_{x_{0} x_{1}}(i \omega)\right]^{++},\left[T^{z_{i}}{ }_{x_{0} x_{2}}(i \omega)\right]^{++}, \ldots\left[T^{z_{i}}{ }_{x_{0} x_{L}}(i \omega)\right]^{++}$para reduzir a quantidade de informação de cada matriz. Isso pode ser feito através de uma avaliação das máximas amplitudes de todas as funções das matrizes $\left[T^{z_{i}}{ }_{x_{0} x_{1}}(i \omega)\right]^{++},\left[T^{z_{i}}{ }_{x_{0} x_{2}}(i \omega)\right]^{++}, \ldots\left[T^{z_{i}}{ }_{x_{0} x_{L}}(i \omega)\right]^{++}$a partir do uso do primeiro valor singular. Esse procedimento é similar ao método FDD aplicado na matriz de densidade espectral visto no Capítulo 3. Assim, a decomposição das matrizes $\left[T^{z_{i}}{ }_{x_{0} x_{1}}(i \omega)\right]^{++},\left[T^{{z_{i}}_{x_{0} x_{2}}}(i \omega)\right]^{++}, \ldots\left[T^{{Z_{i}}_{x_{0} x_{L}}}(i \omega)\right]^{++}$é dada por.

$\left[T^{z_{i}}{ }_{x_{o_{i}} x_{j}}(i \omega)\right]=\left[U^{j}(i \omega)\right]_{L \times L}\left[\Sigma^{j}(i \omega)\right]_{L \times L}\left[V^{j}(i \omega)\right]_{L \times L}{ }^{H}$

Em que, $\left[\Sigma^{j}(i \omega)\right]$ é uma matriz diagonal com $\sigma_{1}^{j}(i \omega) \geq \sigma_{2}^{j}(i \omega) \geq \ldots \geq \sigma_{L}^{j}(i \omega)>0$. Os pólos das funções de primeiros valores singulares $\sigma_{1}^{1}(i \omega), \sigma_{1}^{2}(i \omega), \ldots \sigma_{1}^{L}(i \omega)$ na decomposição de valores singulares de cada matriz conterão pólos iguais aos pólos reais do sistema, da mesma forma como nas matrizes $\left[T^{z_{i}}{ }_{x_{0} x_{1}}(i \omega)\right]^{++} \quad\left[T^{z_{i}}{ }_{x_{0} x_{2}}(i \omega)\right]^{++} \quad \ldots \quad\left[T^{z^{z_{i}} x_{o} x_{L}}(i \omega)\right]^{++}$. No entanto, neste método é proposto o cálculo de uma única função, através de uma média ponderada entre as funções $\sigma_{1}^{1}(i \omega), \sigma_{1}^{2}(i \omega), \ldots \sigma_{1}^{L}(i \omega)$. A média ponderada consiste em multiplicar a amplitude pela mesma amplitude em cada função $\sigma_{1}^{1}(i \omega), \sigma_{1}^{2}(i \omega), \ldots \sigma_{1}^{L}(i \omega)$ e dividir pela soma das

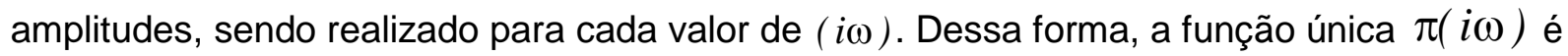
definida assim.

$\pi(i \omega)=\frac{\sum_{j=1}^{L}\left(\sigma_{1}^{j}(i \omega)\right)^{2}}{\sum_{j=1}^{L} \sigma_{1}^{j}(i \omega)}$

O custo computacional para aplicar o método PolyMAX na função $\pi(i \omega)$ é menor do que para aplicar em uma linha ou coluna das matrizes $\left[T^{z_{i} x_{0} x_{1}}(i \omega)\right]^{++},\left[T^{z_{i}}{ }_{x_{0} x_{2}}(i \omega)\right]^{++}, \ldots\left[T^{z_{i} x_{o} x_{L}}(i \omega)\right]^{++}$, obtendo os mesmos parâmetros modais de frequências naturais e taxas de amortecimento. Esse procedimento de simplificação da informação a partir do uso dos primeiros valores singulares pode ser igualmente aplicado no método proposto no Capítulo 5. 
O procedimento para a identificação de modos de vibração é o mesmo dado no Capítulo 4. A partir do uso do primeiro vetor singular $\left\{\hat{U}(i \omega)_{1}\right\}$ na frequência de ressonância, i $\omega_{l}$ para cada matriz de transmissibilidade $\left.\mid T^{Z_{i}}{ }_{o_{i} x_{j}}(i \omega)\right]$, o qual pode ser extraído ao fazer a inversa das matrizes. A média dos diferentes vetores singulares pode fazer uma estimativa da forma modal, como.

$\hat{\hat{U}}\left(i \omega_{l}\right)_{1}=\frac{1}{L} \sum_{j=1}^{L} \hat{U}^{j}\left(i \omega_{l}\right)_{1}$

\subsection{IDENTIFICAÇÃO DO NÚMERO DE CARGAS NÃO CORRELACIONADAS.}

Um importante parâmetro no método proposto neste capítulo e também no Capítulo 5 é o número de valores singulares que deve ser usado na inversa das matrizes de transmissibilidade. O número máximo de valores singulares usado na inversa deve ser igual ao número de cargas não correlacionadas presente na estrutura. No Capítulo 5 o critério proposto para escolher o número de valores singulares foi o uso da média de todos os valores singulares na faixa de frequência de cada valor singular. No entanto, esse procedimento precisa da decisão do usuário do método para escolher o número de valores singulares. Por isso, no presente capítulo é proposto um procedimento automático (não dependente do usuário) de identificação do número de cargas não correlacionadas presentes na estrutura e, portanto, do número máximo de valores singulares que pode ser usado nas inversas das matrizes de transmissibilidade.

O procedimento para a identificação do número de cargas é baseado na reconstrução da matriz de transmissibilidade. Isso pode ser realizado através de sucessivas tentativas de comparação entre a matriz de transmissibilidade reconstruída e a matriz de transmissibilidade original. Cada matriz de transmissibilidade é reconstruída a partir de uma variação incremental do número de valores de singulares que podem ser usados na somatória da Equação (6-3). Dessa forma, o número de cargas é identificado quando a matriz de transmissibilidade reconstruída representa fielmente a matriz de transmissibilidade original para um incremento determinado de valores singulares, ou em termos gerais, quando o erro avaliado entre as duas matrizes for mínimo. O erro mínimo considerado nesse procedimento é de 5\%. Uma ilustração desse procedimento é apresentada na Figura 6-1.

O processo de identificação do número de cargas como descrito na Figura 6-1, pode ser aplicado a cada uma das matrizes de transmissibilidade 
$\left[T^{Z_{i}}{ }_{x_{o} x_{1}}(i \omega)\right], \quad\left[T^{Z_{i}}{ }_{x_{0} x_{2}}(i \omega)\right], \ldots\left[T^{Z_{i}}{ }_{x_{o} x_{L}}(i \omega)\right]$, mas deve ser considerado que o número de cargas identificado em cada matriz deve ser o mesmo. Finalmente, este procedimento poderia ser aplicado sobre a matriz de densidade espectral para identificar a quantidade de cargas não correlacionadas na estrutura, e pode ser usado no método proposto no Capítulo 5 para identificar o número de valores singulares que deve ser usado na inversa da matriz de transmissibilidade.

- Passo 0. Definir os parâmetros inicias 1) matriz de transmissibilidade original $\left[T^{z_{i}}{ }_{x_{o} x_{j}}(i \omega)\right]$ e 2) o número máximo de valores singulares, igual ao tamanho $\mathbf{L}$ da matriz.

- Passo 1. Realizar uma decomposição de valores singulares da matriz de transmissibilidade

$$
\left[T^{z_{i}}{ }_{x_{o_{i}} x_{j}}(i \omega)\right]=[U(i \omega)]_{L \times L}[\Sigma(i \omega)]_{L \times L}[V(i \omega)]_{L \times L}^{H}
$$

- Passo 2. Realizar uma reconstrução da matriz de transmissibilidade, $\left[T^{z_{i}}{ }_{D_{0_{i}} x_{j}}(i \omega)\right]^{R}$ usando $\mathbf{k}$ valores singulares.

$$
\left[T^{z_{i}} x_{o_{i}} x_{j}(i \omega)\right]^{R}=\sum_{i=1}^{k} \sigma_{i}(i \omega)\left\{U(i \omega)_{i}\right\}\left\{V(i \omega)_{i}\right\}^{H}
$$

- Passo 3. Avaliar o erro, $\varepsilon$ entre a matriz de transmissibilidade original e a reconstruída.

$$
\begin{aligned}
& {[\operatorname{Erro}(\%)]_{L x L}=100 \cdot \frac{\left.\sum_{i \omega} \mid\left[\mid T^{z_{i}}{ }_{\alpha_{o_{i}} x_{j}}(i \omega)\right]\right]-\left[\mid T^{z_{i}}{ }_{\alpha_{o_{i}} x_{j}}(i \omega)\right]^{R} \mid}{\left.\sum_{i \omega}\left[\mid T^{z_{i}}{ }_{\alpha_{o_{i}} x_{j}}(i \omega)\right]\right]}} \\
& \varepsilon=\sum_{j=1}^{L} \sum_{i=1}^{L}\left[\operatorname{Erro}_{i, j}\right]
\end{aligned}
$$

- Passo 4. Repetir os passos 2 e 3 incrementando o valor de $\mathbf{k}$ entre 1 e $\mathbf{L}$. O número de cargas é igual ao valor de $\mathbf{k}$ quando se atingir um erro menor que o $5 \%$ ( $\varepsilon \leq 5 \%$ ). Não continuar incrementando $\mathbf{k}$ quando se atingir um erro menor do que $5 \%$.

Figura 6-1 Identificação automática do número de cargas naõ correlacionadas presentes na estrutura.

\subsection{EXEMPLO NUMÉRICO.}

Para demonstrar o desempenho do método PSDTM-SVD melhorado é empregado o mesmo exemplo que tem sido usado nos Capítulos 4 e 5. Nesse exemplo numérico, realizase uma análise da resposta da estrutura sob cargas não correlacionadas em um tempo total de 500 seg. A forças dadas nesse exemplo contêm uma frequência predominante de $30 \mathrm{~Hz}$ 
(ver Figura 5-1) e são localizadas nos graus de liberdade 5, 8 e 11. O produto da análise dinâmica sob carregamento consiste em 5 respostas de acelerações dadas nos graus de liberdade 5,8,11,14 e 17. A frequência de amostragem para excitação da análise dinâmica é de $350 \mathrm{~Hz}$.

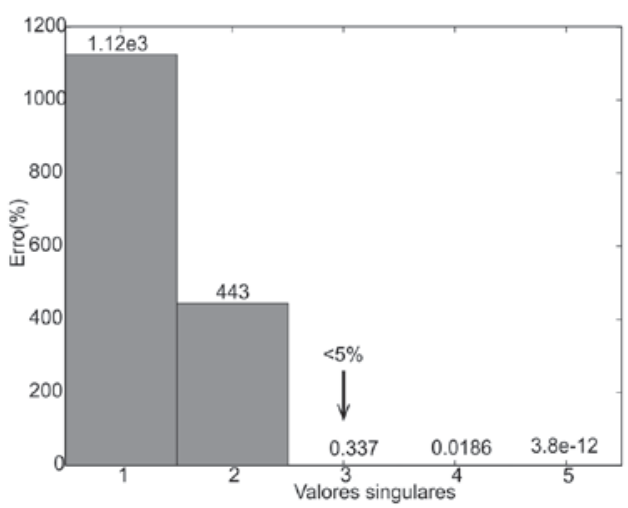

Figura 6-2 Identificação do número máximo de valores singulares na matriz $\left[T^{Z_{i}} x_{o} x_{5}(i \omega)\right]$.

O primeiro passo no método de identificação modal é avaliar as matrizes de transmissibilidade segundo a Equação (6-1). Neste caso, deve-se fazer uma variação do ponto $\boldsymbol{j}$ entre os graus de liberdade 5,8,11,14 e 17, obtendo 5 matrizes de transmissibilidade dadas por $\left.\left[T^{Z_{i}}{ }_{x_{0} x_{5}}(i \omega)\right], \mid T^{z_{i}}{ }_{x_{o} x_{8}}(i \omega)\right],\left[T^{Z_{i}} x_{o} x_{11}(i \omega)\right],\left[T^{Z_{i}}{ }_{x_{o} x_{14}}(i \omega)\right],\left[T^{Z_{i}}{ }_{x_{o} x_{17}}(i \omega)\right]$. A cada uma dessas matrizes de transmissibilidade é empregado o procedimento descrito na Figura 6-1 para identificar o número máximo de valores singulares. Na Figura 6-2 há um exemplo de identificação do número máximo de valores singulares da matriz $\left[T^{z_{i}} x_{0} x_{5}(i \omega)\right]$. Nessa figura se podem notar os erros calculados entre a matriz de transmissibilidade $\left[T^{z_{i}}{ }_{x_{0} x_{5}}(i \omega)\right]$ e diferentes matrizes reconstruídas com $k$ valores singulares, variados entre 1 e 5 . Pode ser verificado que o erro calculado para $\mathrm{k}$ igual a 3 é de $0.337 \%$ sendo menor que o limite de $5 \%$ proposto no procedimento da Figura 6-1. Isso indica que três valores singulares são suficientes para representar a matriz $\left.\mid T^{Z_{i}}{ }_{\alpha_{0} x_{5}}(i \omega)\right]$. Igualmente, três é equivalente ao número de cargas presentes na excitação. Realizando o procedimento para as restantes matrizes foi obtido o mesmo número máximo de valores singulares.

O segundo passo é avaliar a inversa de cada uma das matrizes de transmissibilidade segundo a Equação (6-3). A variável k na Equação (6-3) deve ser igual a 3 segundo a identificação do número máximo de valores singulares realizado no passo anterior. $\mathrm{O}$ 
$\begin{array}{llllll}\text { resultado } & \text { são } & 5 & \text { matrizes } & \text { inversas }\end{array}$ $\left[T^{z_{i}}{ }_{x_{0} x_{5}}(i \omega)\right]^{++},\left[T^{z_{i}}{ }_{x_{0} x_{8}}(i \omega)\right]^{++},\left[T^{z_{i}}{ }_{x_{0} x_{11}}(i \omega)\right]^{++},\left[T^{z_{i}} x_{0} x_{14}(i \omega)\right]^{++},\left[T^{z_{i}} x_{0} x_{17}(i \omega)\right]^{++}$. A partir do cálculo das matrizes inversas, o terceiro passo é aplicar uma decomposição de valores singulares nessas matrizes inversas segundo a Equação (6-4). A decomposição de valores singulares pode avaliar o máximo ganho dessas matrizes, sendo dado pelos primeiros valores singulares, $\sigma_{1}^{5}(i \omega), \sigma_{1}^{8}(i \omega), \sigma_{1}^{11}(i \omega), \sigma_{1}^{14}(i \omega), \sigma_{1}^{17}(i \omega)$. Assim, o passo final é calcular a média ponderada entre os primeiros valores singulares como proposto na Equação (6-6). Dessa forma, na Figura 6-3 a) e b) mostram-se os resultados dos primeiros valores singulares para cada matriz inversa e o resultado da média ponderada entre os primeiros valores singulares, respectivamente. Assim, pode-se notar no resultado final do método apresentado na Figura 6-3 b) a identificação das frequências naturais do sistema sem a introdução da frequência predominante de $30 \mathrm{~Hz}$ usada na excitação.

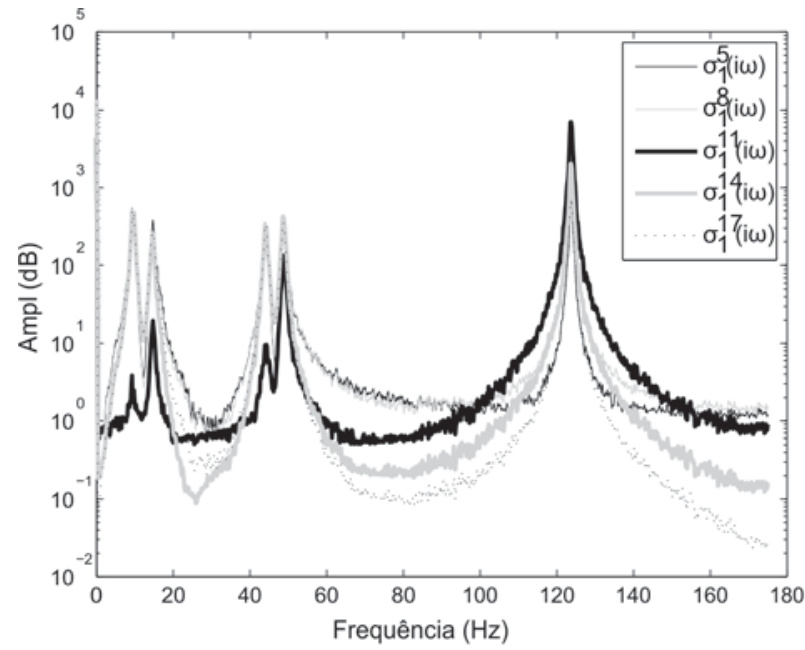

a)

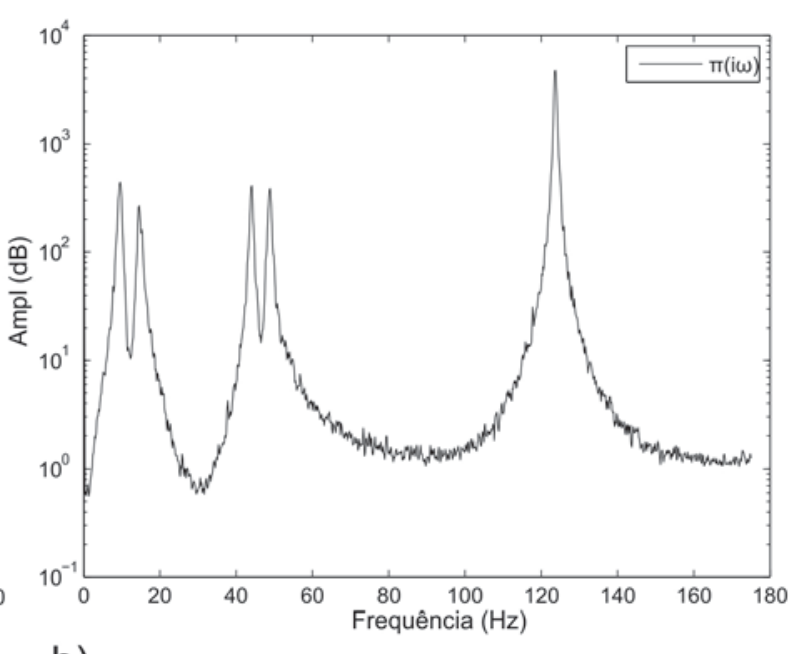

b)

Figura 6-3 a) Primeiros valores singulares das matrizes inversas b) Média ponderada dos primeiros valores singulares.

Uma identificação manual das frequências de ressonância poderia ser diretamente realizada a partir da seleção dos picos da função $\pi(i \omega)$. No entanto, para a identificação das taxas de amortecimento, uma alternativa pode ser modelar a função $\pi(i \omega)$ apresentada na Figura 6-3 b) através do método PolyMAX. Assim, na Figura 6-4 a), mostra-se o diagrama de estabilização do método PolyMAX construído a partir da função da Figura 6-3 b), considerando a ordem dos polinômios de ajuste em uma faixa entre 20 e 180 . Igualmente, na Figura 6-4 b), mostra-se a estabilidade das taxas de amortecimento que foram alcançadas para os pólos estáveis encontrados no diagrama representado na Figura 6-4 a). 


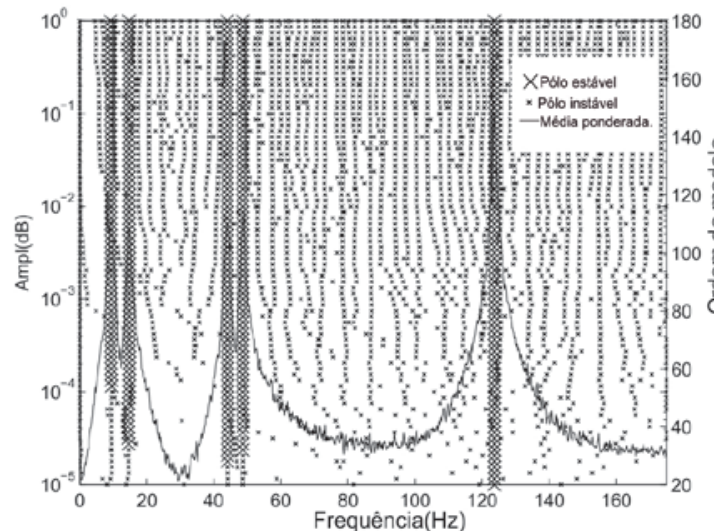

a)

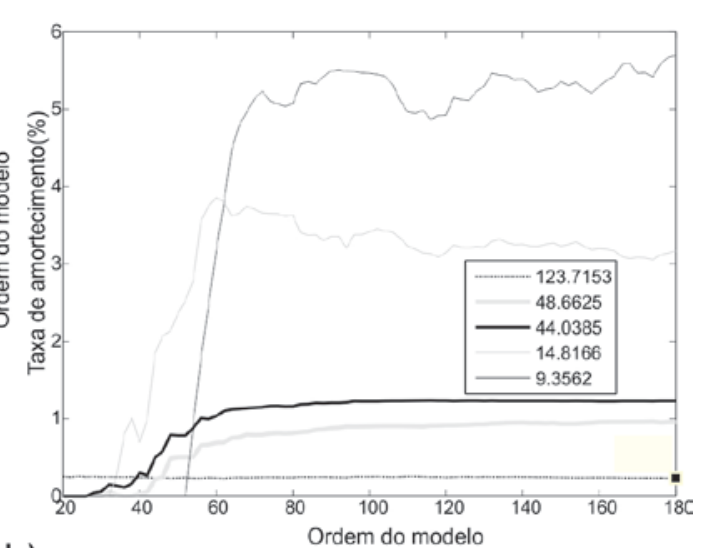

b)

Figura 6-4. a) Diagrama de estabilidade do método PolyMAX aplicado na função $\pi(i \omega)$ b) estabilidade das taxas de amortecimento dos pólos estáveis extraídos da Figura 6-4 a)

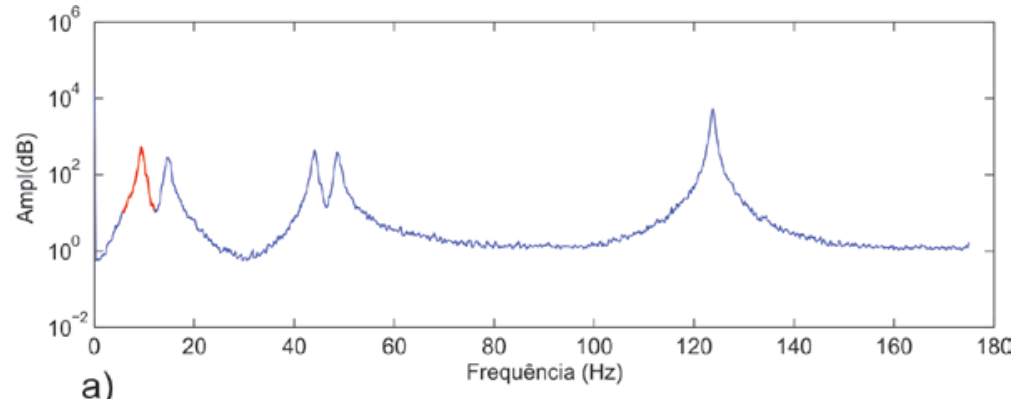

a)

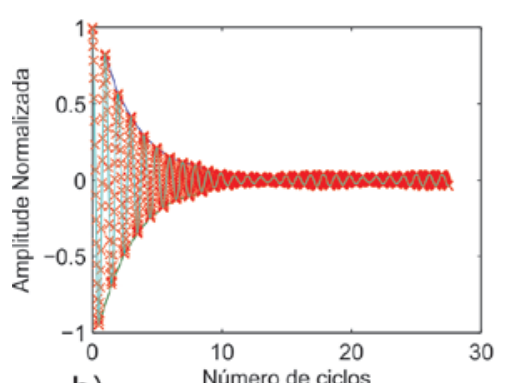

b)

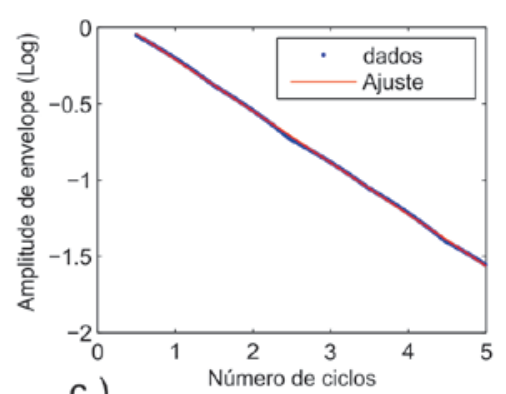

c)

Figura 6-5 a) Faixa de frequências selecionada na função $\pi(i \omega)$ b) Transformada inversa de Fourier da faixa selecionada c) ajuste da envolvente da função em b) para avaliar o decremento logarítmico.

Uma outra alternativa para a identificação das taxas de amortecimentos na função $\pi(i \omega)$ pode ser a aplicação de técnicas clássicas não paramétricas de identificação do amortecimento como o método da largura de meia banda de potência ou o método do decremento logarítmico (Chopra, 2001) entre outros. Neste exemplo, foi usado o método do 
decremento logarítmico para a identificação das taxas de amortecimento. O procedimento básico para aplicar o método do decremento logarítmico na função $\pi(i \omega)$ consiste nos seguintes passos: 1) selecionar as amplitudes de uma faixa de frequências próxima da frequência de ressonância e completar as demais amplitudes da faixa total de frequências com zeros, 2) aplicar a transformada inversa de Fourier nos dados do passo 1 e 3) avaliar o decremento logarítmico na função no domínio do tempo resultante do passo 2. A estimação da taxa de amortecimento por meio do método do decremento logarítmico é influenciada principalmente pela largura da faixa de frequência selecionada próxima da frequência de ressonância e pela resolução em frequência da função $\pi(i \omega)$.Portanto, nesse método, frequências de ressonância muito próximas podem causar erros na estimação do amortecimento. Um exemplo de aplicação do método do decremento logarítmico na função $\pi(i \omega)$ para a estimação do amortecimento é mostrado na Figura 6-5.

A Tabela 6-1 apresenta um resumo das frequências naturais amortecidas e as taxas de amortecimento identificadas tanto com o método PolyMAX como com o método do decremento logarítmico aplicados na função resultante do método PSDTM-SVD melhorado. Os resultados de frequências naturais e de taxas de amortecimentos se aproximam com os valores teóricos. Porém, maiores erros foram encontrados na identificação das taxas de amortecimento. No caso, da estimação dos modos de vibração, os resultados não são apresentados, já que o método propõe o mesmo procedimento exposto no método do Capítulo 4. Portanto, os resultados de modos de vibração obtidos são iguais aos apresentados na Tabela 4-2.

Tabela 6-1 Frequências naturais amortecidas e taxas de amortecimento identificadas usando o método PSDTM-SVD melhorado.

\begin{tabular}{|c|c|c|c|c|c|c|c|c|}
\hline \multirow[b]{3}{*}{ Modos } & \multirow{2}{*}{\multicolumn{2}{|c|}{ Valores teóricos }} & \multirow{2}{*}{\multicolumn{2}{|c|}{$\begin{array}{c}\text { Ordem do modelo } 180 \\
\text { PolyMAX }\end{array}$}} & \multirow{3}{*}{$\begin{array}{c}\begin{array}{c}\text { Decremento } \\
\text { logarítmico }\end{array} \\
\text { Taxas de } \\
\text { amortecimento } \\
(\%)\end{array}$} & \multicolumn{3}{|c|}{ Erro (\%) } \\
\hline & & & & & & \multicolumn{2}{|c|}{ PolyMAX } & \multirow{2}{*}{$\begin{array}{c}\text { Decremento } \\
\text { Taxas de } \\
\text { amortecimento } \\
(\%)\end{array}$} \\
\hline & $\begin{array}{l}\text { Frequências } \\
\text { naturais }(\mathrm{Hz})\end{array}$ & $\begin{array}{c}\text { Taxas de } \\
\text { amortecimento } \\
(\%)\end{array}$ & $\begin{array}{l}\text { Frequências } \\
\text { naturais }(\mathrm{Hz})\end{array}$ & $\begin{array}{c}\text { Taxas de } \\
\text { amortecimento } \\
(\%)\end{array}$ & & $\begin{array}{l}\text { Frequências } \\
\text { naturais }(\mathrm{Hz})\end{array}$ & $\begin{array}{c}\text { Taxas de } \\
\text { amortecimento } \\
(\%)\end{array}$ & \\
\hline 1 & 9.54 & 5.00 & 9.48 & 5.61 & 4.63 & 0.65 & 12.20 & 7.37 \\
\hline 2 & 14.62 & 3.27 & 14.70 & 3.16 & 4.08 & 0.61 & 3.36 & 24.71 \\
\hline 3 & 44.05 & 1.08 & 43.98 & 1.23 & 1.05 & 0.16 & 13.89 & 3.08 \\
\hline 4 & 48.70 & 0.98 & 48.75 & 0.96 & 1.19 & 0.10 & 2.04 & 21.16 \\
\hline 5 & 123.49 & 0.39 & 123.73 & 0.23 & 0.27 & 0.20 & 40.26 & 30.74 \\
\hline
\end{tabular}

\subsection{CONSIDERAÇÕES FINAIS}

No presente capítulo, foi proposta uma melhora do PSDTM-SVD apresentado no Capítulo 4. O método PSTDM-SVD melhorado considera o uso da inversa truncada MoorePenrose sobre as matrizes de transmissibilidade como uma alternativa para preservar a identificação das taxas de amortecimento na função resultante. Uma das variáveis importantes no emprego da inversa truncada Moore-Penrose sobre as matrizes de 
transmissibilidade é a quantidade de valores singulares, parâmetro que é igual ao número de cargas não correlacionadas presente na estrutura. Nesse sentido, o método PSTDM-SVD melhorado propõe uma identificação automática do número de valores singulares para usar na inversa, ao contrário da alternativa proposta no Capítulo 5 que depende da decisão do usuário. Os resultados obtidos na simulação numérica demonstrou que a melhora realizada no método PSDTM-SVD acrescenta a possibilidade da identificação de taxas de amortecimento.

Do mesmo modo que os métodos propostos anteriormente o método proposto neste capítulo tem à impossibilidade de avaliar parâmetros modais quando a excitação encontrase localizada em um só ponto da estrutura. Essa condição de carga gera nas matrizes de

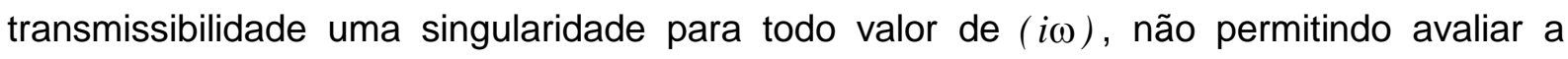
singularidade nos pólos do sistema. Dessa forma, os métodos propostos precisam de pelos menos duas cargas não correlacionadas localizadas em pontos diferentes para obter parâmetros modais. 



\section{CAPÍTULO 7. USO DOS MÉTODOS DE IDENTIFICAÇÃO EM UMA CONDIÇÃO DE UMA ÚNICA CARGA.}

Uma limitação das metodologias de identificação modal apresentadas neste trabalho é a quantidade de cargas não correlacionadas existentes na estrutura. Um mínimo de duas cargas não correlacionadas localizadas em pontos diferentes é necessário para a identificação de parâmetros modais, o que limita a identificação em um caso quando existe uma única carga localizada em um ponto. Isto é devido ao fato que na condição de uma única carga localizada em um ponto, as matrizes de transmissibilidade são singulares para todos os valores de $(i \omega)$. Dessa forma, as estratégias usadas nos métodos propostos baseadas na avaliação da singularidade nos pólos do sistema das matrizes de transmissibilidade ficam inaplicáveis para essa condição carga.

Em uma análise modal de estruturas sob excitações em condições de operação, em que diferentes pontos da estrutura são excitados simultaneamente por diferentes fontes (trafego pedestres e vento) é pouco provável que exista uma condição de uma única carga localizada em um ponto. No entanto, em uma análise modal de uma estrutura em laboratório é comum o uso de uma única fonte de excitação através do uso um shaker o um martelo de excitação. É por isso a necessidade de brindar uma solução para a aplicabilidade dos métodos propostos quando existe uma única fonte de excitação. .

O presente capítulo explora em detalhe a limitação dos métodos de identificação modais propostos quando existe uma única carga localizada em um ponto da estrutura. No entanto, foi demostrado que é possível identificar parâmetros modais quando existe uma única fonte de excitação, sempre e quando essa excitação mude de posição na estrutura. O resultado das funções de transmissibilidade com densidade espectral será o mesmo, usando uma única carga que muda de posição em diferentes pontos ou usando cargas não correlacionadas simultâneas atuando-nos mesmos pontos. Uma simulação numérica é realizada sobre uma viga para demostrar a possibilidade de identificação de parâmetros modais, especificamente em casos quando existe uma única carga que muda de posição. Duas situações de carga única deslocando-se foram consideradas sobre o modelo estrutural: uma carga dinâmica (ruído colorido) e uma carga impulsiva. 


\subsection{TRANSMISSIBILIDADE ESCALAR E MULTIVARIÁVEL COM DENSIDADE ESPECTRAL PARA UMA ÚNICA CARGA.}

No Capítulo 2 é definida a densidade espectral das respostas do sistema em função das funções de transferência $H^{*}{ }_{x_{k} f_{r}}$ ou $H_{x_{j} f_{s}}$ e a densidade espectral das excitações $S_{f_{r} f_{s}}$, assim.

$$
S_{x_{k} x_{j}}(i \omega)=\sum_{r=1}^{N} \sum_{s=1}^{N} H^{*}{ }_{x_{k} f_{r}}(i \omega) \cdot H_{x_{j} f_{s}}(i \omega) \cdot S_{f_{r} f_{s}}(i \omega)
$$

Considerando que as cargas que atuam no sistema são independentes ou nãocorrelacionadas uma representação expandida da Equação (7-1) pode ser dada, como.

$$
\begin{aligned}
S_{x_{k} x_{j}}(i \omega)= & H^{*}{ }_{x_{k} f_{1}}(i \omega) \cdot H_{x_{j} f_{1}}(i \omega) \cdot S_{f_{1} f_{1}}(i \omega)+H^{*}{ }_{x_{k} f_{2}}(i \omega) \cdot H_{x_{j} f_{2}}(i \omega) \cdot S_{f_{2} f_{2}}(i \omega)+\ldots \\
& +H^{*}{ }_{x_{k} f_{N}}(i \omega) \cdot H_{x_{j} f_{N}}(i \omega) \cdot S_{f_{N} f_{N}}(i \omega)
\end{aligned}
$$

No caso da existência de uma única carga localizada em $\boldsymbol{p}$ a densidade espectral da resposta pode ser definida, como.

$$
S_{x_{k} x_{j}}(i \omega)=H^{*}{ }_{x_{k} f_{p}}(i \omega) \cdot H_{x_{j} f_{p}}(i \omega) \cdot S_{f_{p} f_{p}}(i \omega)
$$

A transmissibilidade escalar com densidade espectral se define como uma relação entre duas saídas $x_{o}$ e $x_{j}$, com uma referência de saída $x_{k}$.

$$
T^{k}{ }_{x_{0} x_{j}}(i \omega)=\frac{S_{x_{x_{x} x_{k}}}(i \omega)}{S_{x_{x_{k}} x_{k}}(i \omega)}
$$

Fazendo uso da Equação (7-3) e (7-4) pode ser definida a transmissibilidade escalar com densidade espectral em um caso de uma única carga localizada em $\boldsymbol{p}$ da seguinte forma.

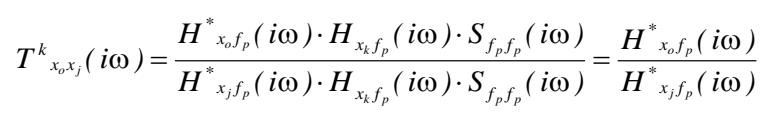

A Equação (7-5) indica que para uma única carga localizada em $\mathbf{p}$ a transmissibilidade escalar com densidade espectral é a mesma qualquer for a referência da saída $x_{k}$. Usando o resultado da Equação (7-5) é possível construir a matriz de transmissibilidade (Equação (4-3)) usada nos métodos de identificação modal dados nos Capítulos 4 e 6 para um caso de uma única carga. 


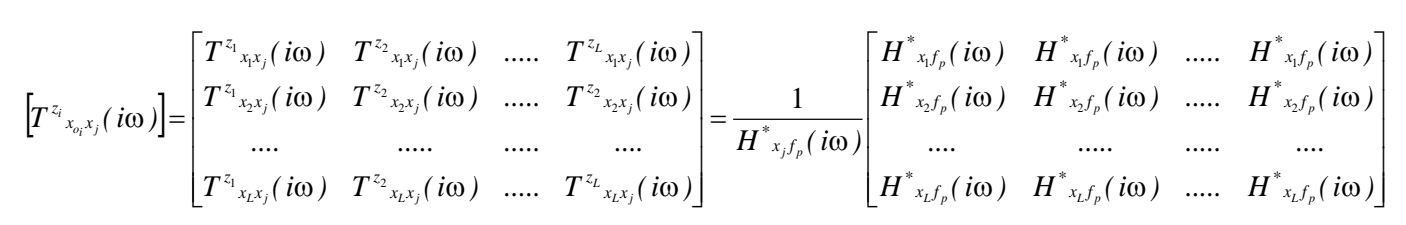

A Equação (7-6) mostra que no caso de uma única carga a matriz de transmissibilidade é singular (linearmente dependente) para qualquer valor de $i \omega$. Os métodos de identificação modal apresentados nos Capítulos 4 e 6 usam como estratégia de identificação modal, a avaliação da singularidade da matriz de transmissibilidade nos pólos do sistema $i \omega_{l}$. Dessa forma, é importante estabelecer que os métodos dados nos Capítulos 4 e 6 não são aplicáveis em um caso de uma única carga localizada em um ponto, já que existe uma singularidade das matrizes de transmissibilidade para todo valor de i $\omega$.

No caso da matriz de transmissibilidade multivariável, ela é definida como uma relação entre a matriz de densidade espectral cruzada entre as respostas nas coordenadas $Z$ e $U$ com a matriz de densidade espectral cruzada entre as respostas nas coordenadas $Z$ e $K$. As coordenadas Z são conhecidas como as referências da saída. Nesse caso a matriz de transmissibilidade também pode ser definida para uma única carga localizada em $\mathbf{p}$ a partir de usar a Equação (7-5) e a Equação (5-12) definindo diferentes referências da saída Z, todas do tamanho $1 \times 1$.

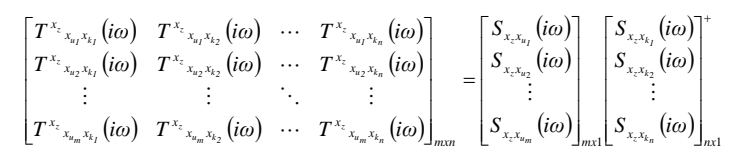

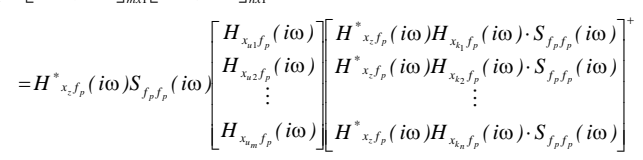

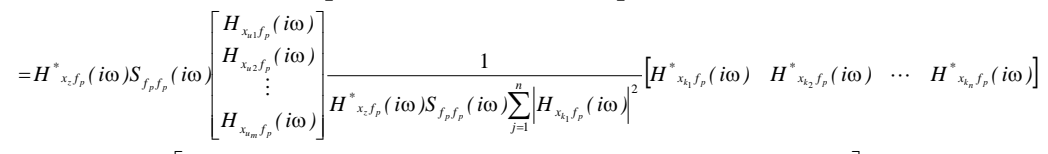

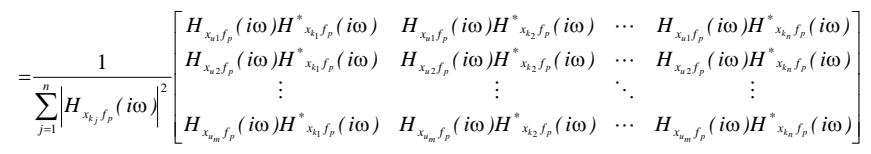

O resultado na Equação (7-7) mostra que para uma condição de uma única carga localizada em um ponto, a matriz de transmissibilidade multivariável será sempre a mesma para qualquer definição de referência da saída $Z$. Dessa forma, também se pode concluir que o método dado no Capítulo 5 não é aplicável em um caso de uma única carga localizada em um ponto, já que o método é baseado na avaliação da singularidade nos pólos do sistema de uma matriz construída a partir de diferentes matrizes de 
transmissibilidade multivariável com diferentes referências da saída. Assim, segundo a Equação (7-7) a matriz usada no método do Capítulo 5 (ver Equação (5-15)) será singular para todos os valores i $\omega$ em uma condição de uma única carga localizada.

\subsection{IDENTIFICAÇÃO EM UMA CONDIÇÂO DE UMA ÚNICA CARGA.}

Como mencionado e demostrado na seção anterior deste capítulo, os métodos identificação propostos neste trabalho não são aplicáveis quando existe uma única carga localizada em ponto da estrutura. No entanto, em uma condição de uma única carga, uma identificação de parâmetros modais com os métodos propostos é possível, sempre e quando a carga mude de posição na estrutura.

$\mathrm{Na}$ Equação (7-2) pode ser notado que a densidade espectral da resposta é calculada pela soma das contribuições de cada uma das cargas que atuam na estrutura. Essa contribuição pode ser efetuada de duas formas: com cargas atuando simultaneamente em cada ponto da estrutura ou pela ação de uma única carga que muda de posição em diferentes intervalos de tempo. O resultado pelas duas formas é o mesmo. Portanto, a densidade espectral da resposta pode-se definir também como uma soma de cargas localizadas em um ponto, mas mudando de posição em diferentes intervalos de tempo.

$$
\begin{aligned}
& S_{x_{k} x_{j}}(i \omega)=S_{x_{k} x_{j}}(i \omega)_{f_{1} t_{1}}+S_{x_{k} x_{j}}(i \omega)_{f_{2} t_{2}}+\ldots+S_{x_{k} x_{j}}(i \omega)_{f_{N} t_{N}} \\
& \text { Com, } \\
& S_{x_{k} x_{j}}(i \omega)_{f_{1} t_{1}}=H^{*}{ }_{x_{k} f_{1}}(i \omega) \cdot H_{x_{j} f_{1}}(i \omega) \cdot S_{f_{1} f_{1}}(i \omega) \\
& S_{x_{k} x_{j}}(i \omega)_{f_{2} t_{2}}=H^{*}{ }_{x_{k} f_{2}}(i \omega) \cdot H_{x_{j} f_{2}}(i \omega) \cdot S_{f_{2} f_{2}}(i \omega) \\
& S_{x_{k} x_{j}}(i \omega)_{f_{N} t_{N}}=H^{*}{ }_{x_{k} f_{N}}(i \omega) \cdot H_{x_{j} f_{N}}(i \omega) \cdot S_{f_{N} f_{N}}(i \omega)
\end{aligned}
$$

Em que, $S_{x_{k} x_{j}}(i \omega)_{f_{1} t_{1}}$ é a contribuição de uma carga localizada na coordenada 1 , atuando no intervalo de tempo $1, S_{x_{k} x_{j}}(i \omega)_{f_{2} t_{2}}$ é a contribuição de uma carga localizada na coordenada 2, atuando no intervalo de tempo 2 e $S_{x_{k} x_{j}}(i \omega)_{f_{N} t_{N}}$ é a contribuição de uma carga localizada na coordenada $N$, atuando no intervalo de tempo $N$. Na Equação (7-8) em cada intervalo de tempo existe uma condição de carga diferente, dada por uma carga localizada atuando em um ponto só. Por exemplo, no primer intervalo de tempo $t_{1}$, a carga é somente aplicada no ponto 1 . No final do intervalo de tempo $t_{1}$, e no começo e durante o 
intervalo de tempo $t_{2}$ a carga é somente aplicada no ponto 2. Dessa forma, cada um desses intervalos de tempo está associado a cada contribuição de carga na Equação (7-8) .

Como exemplo, é realizado um cálculo teórico da função de transmissibilidade escalar $T_{x_{5} x_{8}}^{11}(i \omega)=S_{x_{5} x_{11}}(i \omega) / S_{x_{8} x_{11}}(i \omega)$ sobre a estrutura mostrada na Figura 7-1, considerando as seguintes situações de carga: 1) duas cargas (ruído branco) localizadas nos graus de liberdade 5 e 8, as quais atuam simultaneamente (ver Figura 7-1) e 2) duas cargas (ruído branco) localizadas nos graus liberdade 5 e 8, mas que atuam em intervalos de tempo diferentes (ver Figura 7-2). A segunda situação pode-se entender como um caso de uma única carga que muda de posição, tendo uma carga que atua primeiro no grau de liberdade 5 e depois passa a atuar no grau de liberdade 8.

O resultado do cálculo teórico confirma que a função de transmissibilidade $T_{x_{5} x_{8}}^{11}(i \omega)=S_{x_{5} x_{11}}(i \omega) / S_{x_{8} x_{11}}(i \omega)$ é a mesma para ambas as configurações de carga, como mostrado nas Figura 7-1 e 7-2. Isso indica que uma única carga mudando de posição em dois pontos diferentes é equivalente a ter duas cargas atuando simultaneamente nos mesmos pontos. Igualmente, se a carga única mudasse de posição em 4 pontos seria equivalente a ter 4 cargas atuando simultaneamente. Assim, pode-se concluir que os métodos de identificação modal apresentados neste trabalho continuam sendo aplicáveis no caso de uma condição de uma única carga que muda de posição.

É importante esclarecer que para a avaliação das funções de transmissibilidade no caso de uma única carga que muda de posição não é necessário identificar o momento quando a carga muda de posição, já que o cálculo da densidade espectral é definido como uma média de densidades espectrais calculadas em diferentes janelas temporais do sinal (método de Welch), o que sugere um espectro total dado por uma soma de espectros que contêm a contribuição de cada uma das cargas localizadas, exatamente como estabelecido na Equação (7-8) . 

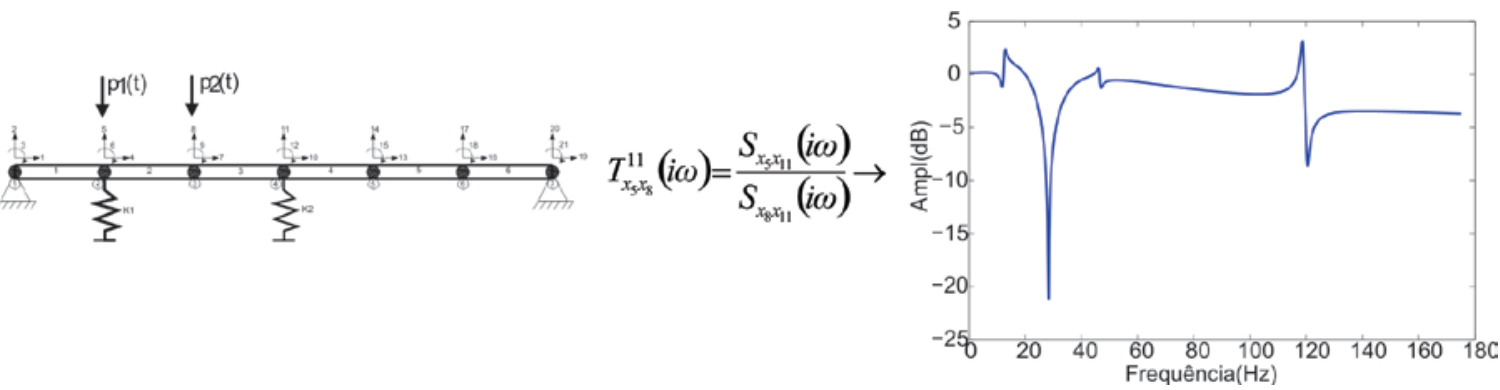

Figura 7-1 Cargas atuando simultaneamente no sistema.
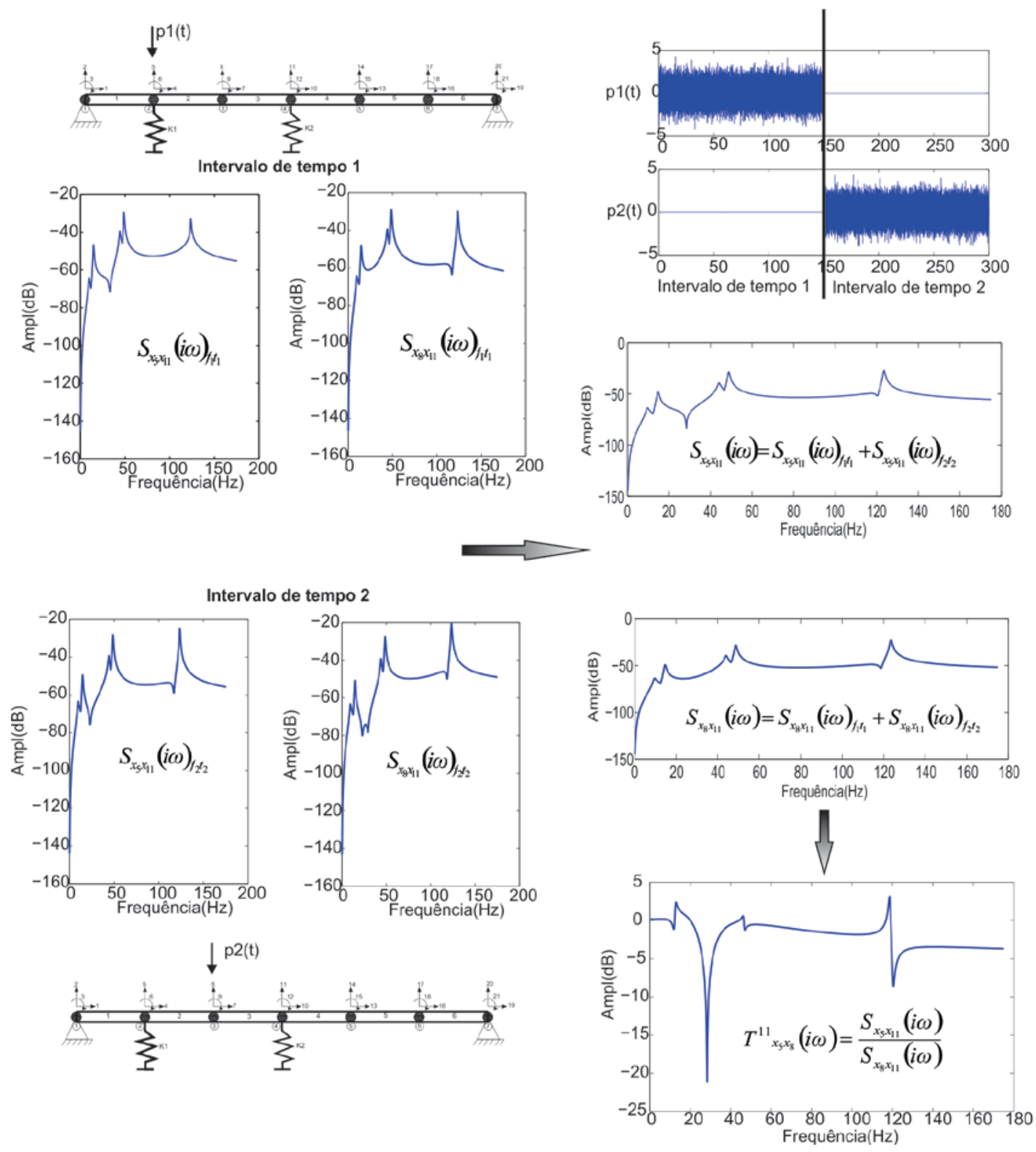

Figura 7-2 Carga única mudando de posição no sistema. 


\subsection{EXEMPLOS NUMÉRICOS COM UMA ÚNICA CARGA.}

O seguinte exemplo numérico pretende demostrar a identificação de parâmetros modais com os métodos de identificação modal propostos, especificamente em casos quando existe uma única carga que muda de posição. O mesmo modelo estrutural que tem sido utilizado nos capítulos anteriores é usado aqui para validar a identificação modal nessas condições de carga. Assim, duas situações de carga única são consideradas sobre o modelo estrutural proposto: Carga 1, com uma carga dinâmica que se desloca entre os graus de liberdade 5, 8 e 11 em intervalos de tempo de 100seg, como mostrado na Figura 7-3, Carga 2 , com uma carga impulsiva que se desloca em cada grau de liberdade vertical a cada 60 seg, como é mostrado na Figura 7-4 . Na situação de Carga 1, as cargas contêm uma frequência predominante de $30 \mathrm{~Hz}$, com um espectro de densidade espectral como mostrado na Figura 5-1. Deve-se ter presente que as duas situações de carga representam uma única carga que se desloca ao longo da estrutura.
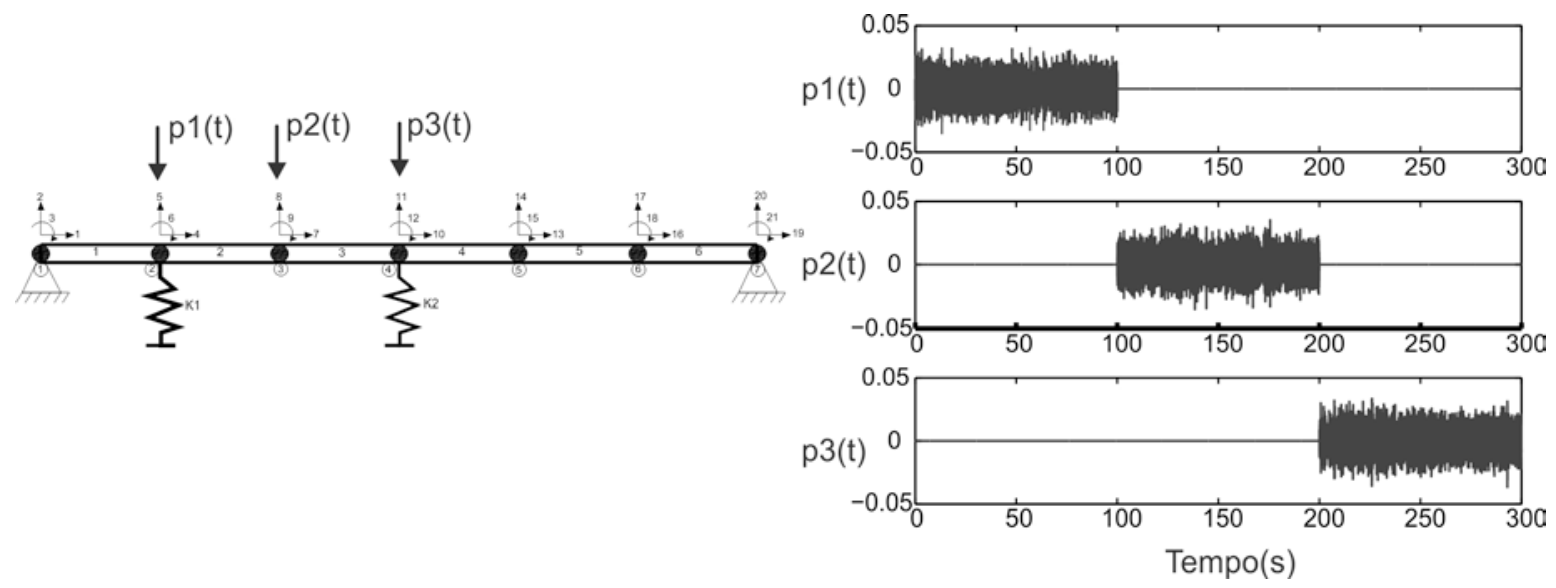

Figura 7-3 Cargas deslocandose entre os graus de liberdade 5,8 e 11, Carga 1.

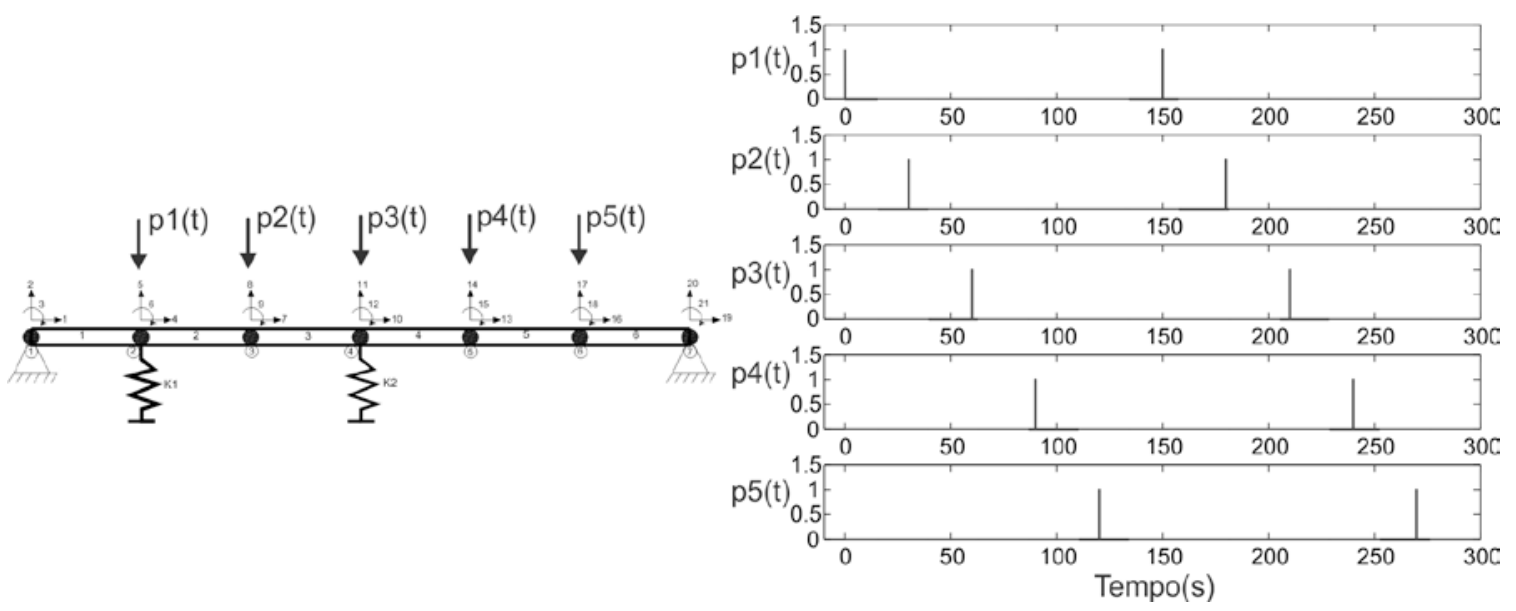

Figura 7-4 Carga impulsiva deslocando-se entre os graus de liberdade verticais, Carga 2. 
Para a identificação de parâmetros modais neste exemplo os métodos usados foram os métodos propostos nos Capítulos 5 e 6 . Cada um dos parâmetros de entrada nestes métodos foi configurado como segue.

a) Método com transmissibilidade multivariável: A configuração das coordenadas de U e $\mathrm{K}$ foram definidas com os graus de liberdade para $U=5,8,11,14,17$ e $K=17$. Com está configuração de coordenadas de $\mathrm{K}$ e U, uma matriz única é obtida igual à dada na Equação (5-24). Para o cálculo dessa matriz, funções de densidade espectral de potência (ver Equação (5-22)) foram avaliadas usando o método de Welch com uma janelas temporais do tamanho $2^{11}$, uma sobreposição do $50 \%$ e um tamanho da transformada de Fourier de $2^{11}$. 0 tipo de janela foi variado, entre uma janela tipo Hanning para o caso de Carga 1 e uma janela tipo retangular para o caso de Carga 2 (Carga impulsiva).

b) Método PSDTM-SVD melhorado. Para avaliação das diferentes matrizes de transmissibilidade que usa este método (Ver Equação (6-1)), funções de densidade espectral de potência foram avaliadas usando o método de Welch com os mesmos parâmetros definidos no método com transmissibilidade multivariável.

Ambos os métodos do Capítulo 5 e 6 usaram uma inversa modificada Moore-Penrose. Nesta inversa deve-se definir a quantidade máxima $k$ de valores singulares, como estabelecido na Equação (6-3). Portanto, foi aplicado em ambos os métodos de identificação o procedimento automático de identificação do número máximo de valores singulares, como proposto Na Figura 6-1. As matrizes de entrada ao método de identificação automático do número de valores singulares foram dadas pelas Equações (5-24) e (6-1) para o método com transmissibilidade multivariável e o método PSDTM-SVD melhorado, respectivamente.

Em ambos os métodos, o número máximo de valores singulares identificado foi de 3 e 5 para o caso de Carga 1 e 2, respectivamente. É importante indicar que o número máximo de valores singulares pode ser relacionado com o número de cargas existente na estrutura. Portanto, o resultado manifesta que na condição Carga 1 e 2 existem 3 e 5 cargas aplicadas, respetivamente. Isso mostra a similitude entre aplicar uma única carga deslocada em diferentes pontos e aplicar cargas simultâneas nos mesmos pontos. Dessa forma, nas inversas das Equações (5-24) e (6-1) foi usado um número máximo de valores singulares de 3 e 5 para o caso de carga 1 e 2, respectivamente.

Os resultados da aplicação dos métodos de identificação do Capítulo 5 e 6 nos casos de carga descritos neste capítulo, como Carga 1 e 2 são apresentados nas Figuras 7-5, 7-6, 7-7 e 7-8. Nas funções resultantes de ambos os métodos de identificação foi aplicado o método PolyMAX para a identificação das taxas de amortecimento. Assim, no caso do 
método com transmissibilidade multivariável foi usado o método PolyMAX sobre a primeira linha da matriz inversa da Equação (5-24) e no caso do método PSDTM-SVD melhorado foi usado o método PolyMAX na função resultante $\pi(i \omega)$ (ver Equação (6-6)). Na parte a) das Figuras 7-5, 7-6, 7-7 e 7-8 são mostrados os diagramas de estabilização do método PolyMAX considerando a ordem dos polinômios de ajuste em uma faixa entre 20 e 120 . Igualmente, na parte b) destas figuras mostra-se a estabilidade das taxas de amortecimento que foram alcançadas para os pólos estáveis encontrados.

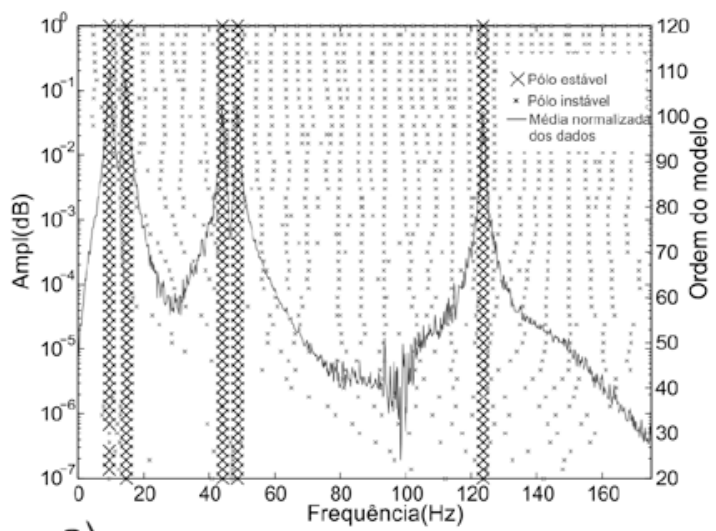

a)

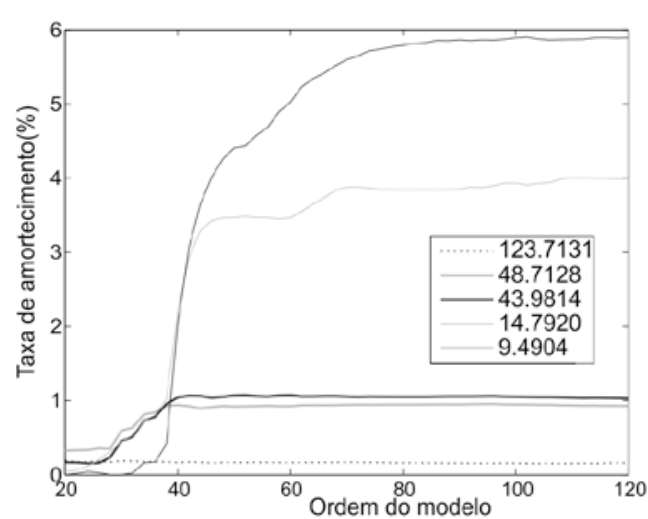

b)

Figura 7-5 a) diagrama de estabilidade e b) estabilidade das taxas de amortecimento para os pólos estáveis determinados usando o método PolyMAX na matriz inversa da Equação 5-24 (método com transmissibilidade multivariável). Caso de Carga 1.

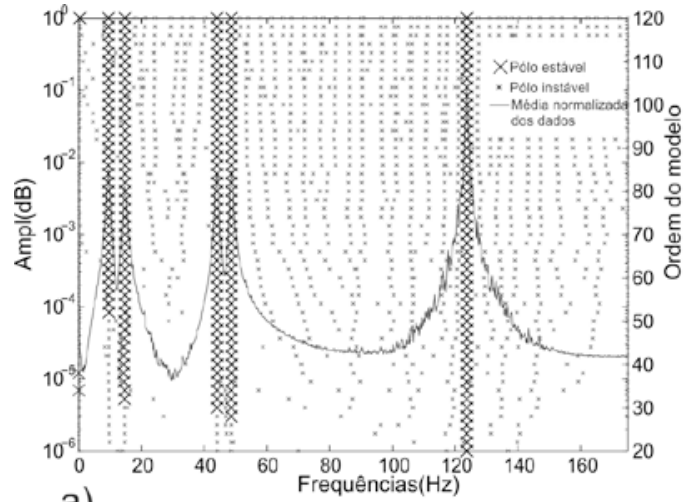

a)

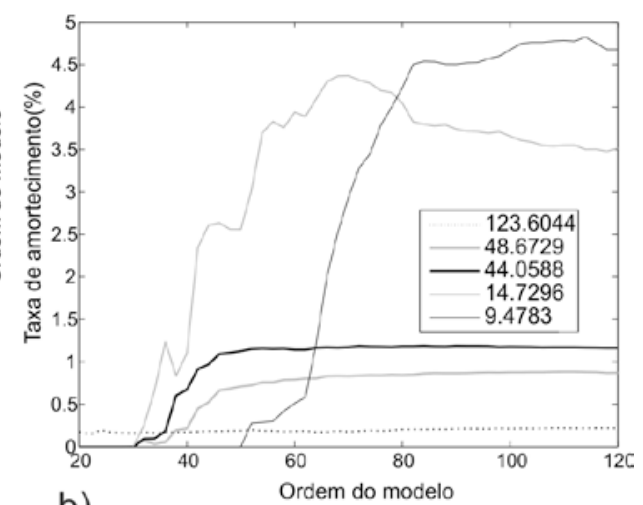

b)

Figura 7-6 a) diagrama de estabilidade e b) estabilidade das taxas de amortecimento para os pólos estáveis determinados usando o método PolyMAX na matriz inversa na matriz inversa da Equação 5-24 (método com transmissibilidade multivariável). Caso de Carga 2. 


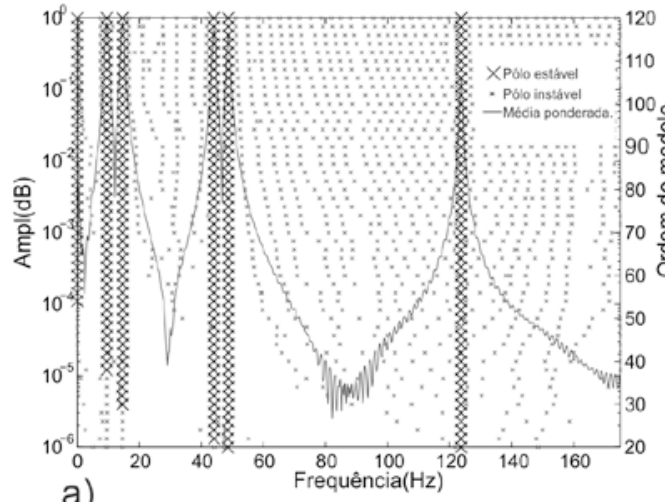

a)

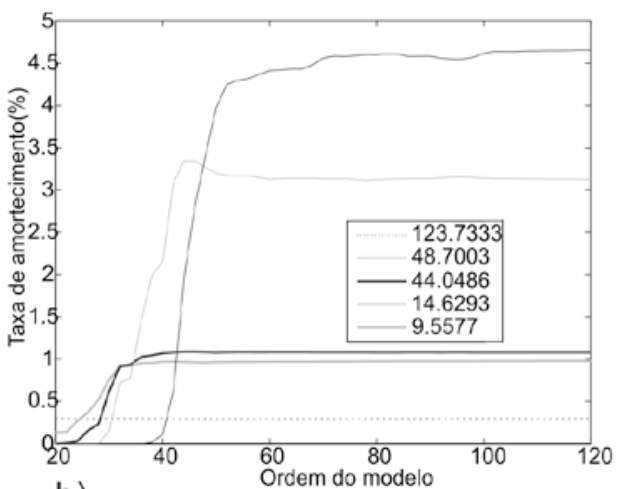

b)

Figura 7-7 a) Diagrama de estabilidade do método PolyMAX aplicado na função resultante do métodos PSDTM-SVD melhorado e b) estabilidade das taxas de amortecimento dos pólos estáveis. Caso de Carga 1.

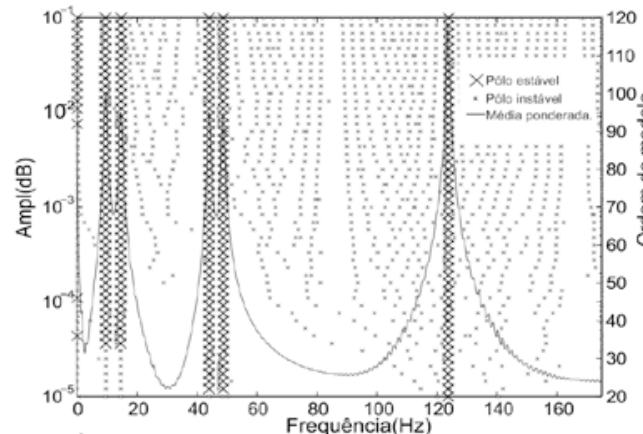

a)

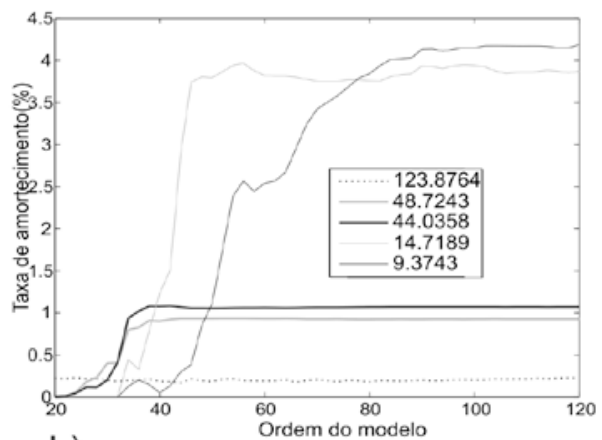

b)

Figura 7-8 a) Diagrama de estabilidade do método PolyMAX aplicado na função resultante do métodos PSDTM-SVD melhorado e b) estabilidade das taxas de amortecimento dos pólos estáveis. Caso de Carga 2.

Nas Tabela 7-1 e 7-2 são apresentados os resultados da identificação das frequências naturais e taxas de amortecimento para os dois casos de carga única (Carga 1 e 2). Assim, Na Tabela 7-1 e 7-2 encontram-se os resultados para o método com transmissibilidade multivariável e o método PSDTM-SVD melhorado, respectivamente. Os resultados de frequências naturais e de taxas de amortecimentos encontrados por ambos os métodos de identificação modal mostraram que são próximos com os valores teóricos em ambos os casos de carga. Dessa forma, pode-se concluir que os métodos de identificação modal propostos neste trabalho baseado no uso da transmissibilidade são aplicáveis em um caso de uma única carga, mas sempre e quando essa carga se desloque de posição. 
Tabela 7-1 Frequências naturais amortecidas e taxas de amortecimento identificadas usando o método com transmissibilidade multivariável.

\begin{tabular}{|c|c|c|c|c|c|c|}
\hline \multirow{3}{*}{ Modos } & \multirow{2}{*}{\multicolumn{2}{|c|}{ Valores teóricos }} & \multicolumn{4}{|c|}{ Ordem do modelo 100 PolyMAX } \\
\hline & & & \multicolumn{2}{|r|}{ Carga 1} & \multicolumn{2}{|r|}{ Carga 2} \\
\hline & $\begin{array}{l}\text { Frequências } \\
\text { naturais }(\mathrm{Hz})\end{array}$ & $\begin{array}{c}\text { Taxas de } \\
\text { amortecimento (\%) }\end{array}$ & $\begin{array}{l}\text { Frequências } \\
\text { naturais }(\mathrm{Hz})\end{array}$ & $\begin{array}{c}\text { Taxas de } \\
\text { amortecimento (\%) }\end{array}$ & $\begin{array}{l}\text { Frequências } \\
\text { naturais }(\mathrm{Hz})\end{array}$ & $\begin{array}{c}\text { Taxas de } \\
\text { amortecimento (\%) }\end{array}$ \\
\hline 1 & 9.54 & 5.00 & 9.49 & 5.89 & 9.48 & 4.62 \\
\hline 2 & 14.62 & 3.27 & 14.79 & 3.93 & 14.73 & 3.14 \\
\hline 3 & 44.05 & 1.08 & 43.98 & 1.05 & 44.06 & 1.08 \\
\hline 4 & 48.70 & 0.98 & 48.71 & 0.95 & 48.67 & 0.98 \\
\hline 5 & 123.49 & 0.39 & 123.71 & 0.16 & 123.60 & 0.29 \\
\hline
\end{tabular}

Tabela 7-2 Frequências naturais amortecidas e taxas de amortecimento identificadas usando o método PSDTM-SVD melhorado.

\begin{tabular}{|c|c|c|c|c|c|c|}
\hline \multirow[b]{3}{*}{ Modos } & \multirow{2}{*}{\multicolumn{2}{|c|}{ Valores teóricos }} & \multicolumn{4}{|c|}{ Ordem do modelo 100 PolyMAX } \\
\hline & & & \multicolumn{2}{|r|}{ Carga 1} & \multicolumn{2}{|c|}{ Carga 2} \\
\hline & $\begin{array}{l}\text { Frequências } \\
\text { naturais }(\mathrm{Hz})\end{array}$ & $\begin{array}{c}\text { Taxas de } \\
\text { amortecimento (\%) }\end{array}$ & $\begin{array}{l}\text { Frequências } \\
\text { naturais }(\mathrm{Hz})\end{array}$ & $\begin{array}{c}\text { Taxas de } \\
\text { amortecimento (\%) }\end{array}$ & $\begin{array}{l}\text { Frequências } \\
\text { naturais }(\mathrm{Hz})\end{array}$ & $\begin{array}{c}\text { Taxas de } \\
\text { amortecimento (\%) }\end{array}$ \\
\hline 1 & 9.54 & 5.00 & 9.56 & 4.67 & 9.37 & 4.15 \\
\hline 2 & 14.62 & 3.27 & 14.63 & 3.65 & 14.72 & 3.94 \\
\hline 3 & 44.05 & 1.08 & 44.05 & 1.18 & 44.04 & 1.07 \\
\hline 4 & 48.70 & 0.98 & 48.70 & 0.88 & 48.72 & 0.92 \\
\hline 5 & 123.49 & 0.39 & 123.73 & 0.21 & 123.88 & 0.20 \\
\hline
\end{tabular}

\subsection{CONSIDERAÇÕES FINAIS}

Neste capítulo uma análise das funções de transmissibilidade com densidade espectral (escalar e multivariável) foi realizado para um caso quando existe uma única carga localizada em um ponto da estrutura. A análise concluiu que para esse caso de carga as funções de transmissibilidade com densidade espectral não dependem da referência da saída. Portanto, qualquer matriz de transmissibilidade contendo diferentes referências da saída nas colunas é singular para todos os valores de $i \omega$. Esse fato gera a impossibilidade de avaliar a singularidade das matrizes nos pólos do sistema, não permitindo avaliar os parâmetros modais. No entanto, em uma condição de uma única fonte de excitação é possível identificar os parâmetros modais, sempre e quando a excitação se desloque de posição. Uma simulação numérica sobre uma viga, usando cargas com uma única fonte de excitação, mas deslocando-se através da estrutura demostrou a possibilidade da identificação de parâmetros modais para essa condição de carga.

Para uma correta identificação dos parâmetros modais, os métodos de identificação modal propostos neste trabalho precisam da existência de múltiplas cargas não correlacionadas atuando na estrutura ou de uma única carga deslocando-se na mesma. No caso de uma única carga que muda de posição seria equivalente a ter múltiplas condições 
de carga. Nesse último aspecto é necessário enfatizar que os métodos propostos não precisam da identificação do momento quando existe uma condição de carga diferente. Isso oferece uma grande vantagem em comparação com outros métodos de identificação modal baseados em transmissibilidade que usam uma mudança de posição da carga para a identificação de parâmetros modais. Esses métodos avaliam diferentes funções de transmissibiildade para cada condição de carga, gerando dessa forma a necessidade de identificar o momento quando ocorre essa mudança de posição da excitação (Devriendt e Guillaume, 2007; 2008; Devriendt, De Sitter e Guillaume, 2010; Devriendt et al., 2013; Weijtjens, De Sitter, et al., 2014; Weijtjens, Lataire, et al., 2014). 


\section{CAPÍTULO 8. CONCLUSÕES E TRABALHOS FUTUROS}

\subsection{CONCLUSÕES}

Este trabalho focaliza o uso de funções de transmissibilidade para a identificação de parâmetros modais (frequências naturais, taxas de amortecimento e modos de vibração). $O$ trabalho apresenta o desenvolvimento e aplicação de três metodologias de identificação modal baseadas no uso de funções de transmissibilidade com densidade espectral. Os métodos propostos foram validados através de simulações numéricas e o uso de dados de vibração de testes desenvolvidos sobre a ponte Garcia Cadena (Bucaramanga-Colômbia).

O uso de funções de transmissibilidade para a identificação modal é uma área de recente pesquisa. A principal inovação desta nova alternativa é que os métodos propostos podem identificar parâmetros modais sem fazer suposição alguma sobre as forças de excitação. Tradicionalmente, os métodos de identificação modal para análise modal operacional supõem que as excitações são do tipo ruído branco. Esta vantagem gera a possibilidade de fazer uma direta separação das frequências naturais do sistema dos possíveis componentes harmônicos existentes na excitação. As primeiras pesquisas nesta área propõem o uso de funções de transmissibilidade a partir de diferentes condições de carga. No entanto, em um caso real, este enfoque possui a necessidade de fazer um préprocessamento da informação para identificar a mudança do carregamento. Esta dificuldade foi superada a partir do uso de funções de transmissibilidade com densidade espectral. Este tipo de funções transmissibilidade é o fundamento dos diferentes métodos de identificação modal propostos neste trabalho.

Desta forma, o desenvolvimento da presente pesquisa permitiu alcançar as seguintes conclusões principais, que sintetizam as conclusões parciais dadas anteriormente no final de cada capítulo.

- Os três métodos propostos, o método PSDTM-SVD, o método com uso da transmissibilidade multivariável com diferentes referências da saída e o método PSDTMSVD melhorado mostram que possuem uma independência do espectro da excitação. Portanto, esses métodos podem ser aplicados para as excitações com um espectro de frequências predominantes (ruído colorido), sempre e quando as frequências do sistema sejam excitadas. Diferentes simulações numéricas a partir de usar forças não correlacionadas com uma frequência predominante mostraram que os métodos puderam fazer uma identificação dos parâmetros modais do sistema sem a introdução da frequência predominante estabelecida previamente na excitação. Além disso, os 
métodos mostraram a possibilidade de fazer uma identificação modal a partir de uma única condição de carga ao invés de diferentes condições de carga como proposto em previas metodologias que usam transmissibilidade.

- O primeiro método proposto, o método PSDTM-SVD permite reduzir o risco de estabelecer frequências de ressonância que não são do sistema em comparação com o método proposto por Yan e Ren, 2012. O método de Yan e Ren não garantia em alguns casos de carga uma correta identificação das frequências de ressonância, devido a uso de funções de subtração inversa entre funções de transmissibilidade com diferentes referências da saída que podiam conter ressonâncias não relacionadas ao sistema. Dessa forma, a proposta do método PSDTM-SVD baseada na avaliação da singularidade nos pólos do sistema de matrizes de transmissibilidade mostra-se como uma alternativa segura para a avaliação de parâmetros modais com uso de funções de transmissibilidade com densidade espectral.

- Uma extensão do conceito de função de transmissibilidade com densidade espectral é proposto neste trabalho, o qual permite uma relação entre múltiplas respostas ao invés de só duas. Esta extensão é chamada como de funções de transmissibilidade multivariável com diferente referência da saída. As funções de transmissibilidade multivariável propostas permitem encontrar uma propriedade nos pólos do sistema do mesmo modo que as funções de transmissibilidade escalar com densidade espectral. Esta propriedade define que as matrizes de transmissibilidade multivariável com as mesmas respostas em relação, mas com diferente referência da saída convergem para a mesma matriz quando se aproximam aos pólos do sistema. Isso indica que as funções de transmissibilidade multivariável propostas podem ser usadas do mesmo modo que as funções de transmissibilidade escalar para a identificação de parâmetros modais do sistema.

A partir do uso da propriedade encontrada nas funções de transmissibilidade multivariável um procedimento para a identificação de parâmetros modais foi proposto. Igualmente, como no método PSDTM-SVD o procedimento foi baseado na construção de uma matriz com singularidade nos pólos do sistema. No entanto, uma forma diferente de avaliação da singularidade foi realizada através da inversa generalizada MoorePenrose. Uma modificação da inversa generalizada baseada no truncamento do número de valores singulares foi proposta, devido a que a inversa generalizada apresenta resultados corretos somente quando existem cargas não correlacionadas em todos os graus liberdade medidos. O número de valores singulares que deve ser utilizada na inversa é diretamente relacionado com o número de cargas não correlacionadas existentes na estrutura. 
Uma combinação entre o método PolyMAX e o resultado obtido da inversa modificada na matriz construída de diferentes funções de transmissibilidade multivariável demostrou que é possível modelar a inversa desta matriz com o fim de avaliar as frequências naturais e as taxas de amortecimento. Adicionalmente, o uso das funções de transmissibilidade multivariável permite avaliar os mesmos parâmetros modais a partir de uma variação das respostas em relação. Isso pode criar uma forma de validação dos parâmetros modais identificados através da comparação de diferentes resultados de diferentes relações entre respostas.

- O método PSDTM-SVD melhorado acrescenta a possibilidade de identificação das taxas de amortecimento. A proposição desta melhora é devida a que no método PSDTM-SVD original foi gerada uma alteração do amortecimento modal pelas operações matemáticas propostas entre valores singulares. Dessa forma, o método PSDTM-SVD melhorado faz uso da inversa modificada para a avaliação da singularidade das matrizes de transmissibilidade. No entanto, uma das variáveis importantes no uso da inversa modificada é a quantidade de valores singulares que devem ser escolhidos. Desse modo, o método PSDTM-SVD melhorado propõe uma identificação automática do número de valores singulares, a qual não depende do usuário.

- Uma limitação das metodologias de identificação modal proposta neste trabalho é a quantidade de cargas não correlacionadas presentes na estrutura. Um mínimo de duas cargas não correlacionadas localizadas em pontos diferentes é necessário para a identificação de parâmetros modais. No entanto, em uma condição de uma única fonte de excitação é possível identificar parâmetros modais, sempre e quando a excitação se desloque de posição. Apesar dos métodos proposto precisarem de múltiplas cargas não correlacionadas atuando na estrutura o de uma única carga deslocando-se, os métodos não precisam da identificação do momento quando a carga muda de posição como é necessário em outros métodos de identificação modal baseados em transmissibilidade.

\subsection{TRABALHOS FUTUROS}

O trabalho apresentado nesta tese inclui três metodologias de identificação modal baseadas no uso de funções de transmissibilidade. No entanto, mais pesquisas são necessárias para melhorar a identificação nos métodos propostos. Adicionalmente, outras 
áreas de aplicação, além da identificação modal podem ser motivo de pesquisa com o uso das funções de transmissibilidade, assim os seguintes aspectos podem ser explorados.

- O custo computacional dos métodos propostos é maior em comparação com outras metodologias não paramétricas no domínio da frequência, devido a que os procedimentos envolvem o cálculo de um número de matrizes de transmissibilidade que é diretamente proporcional ao número de medições. Em um caso em que uma grande quantidade de medições seja realizada simultaneamente, os tempos de cálculo destas metodologias poderiam tornar-se proibitivos. Devido a isso, é necessário pesquisar uma forma de reduzir a quantidade de informação que é utilizada, de tal forma que não afete a informação modal do sistema.

- Embora as metodologias fossem aplicadas em um caso de vibrações real mostrando bons resultados, a maioria das aplicações de estruturas de engenharia civil apresentam condições de identificação adversas como, modos vibração muitos próximos e sinais de relação ruído/sinal muito alto. Estas condições de identificação podem se tornar em uma limitação das metodologias propostas do mesmo modo que acontece em outras metodologias no domínio da frequência. É por isso, que uma possibilidade para melhorar a identificação nestas condições é aplicar técnicas de pré-processamento do sinal que possam realizar uma separação do sinal em componentes modais, o qual gera uma identificação de parâmetros modais em sinais separados e, portanto, uma redução do ruído no sinal.

- Diferentes áreas de pesquisa incluindo a área de identificação modal precisam de estudos de confiabilidade. A identificação modal com uso de funções de transmissibilidade oferece e facilita ainda mais a possibilidade de fazer uma análise de confiabilidade dos parâmetros modais identificados, já que diferentes matrizes de transmissibilidade fornecem os mesmos parâmetros modais. Dessa forma avaliando os mesmos parâmetros modais em diferentes matrizes de transmissibilidade pode-se obter para todos os parâmetros modais tanta informação como pontos de medição.

- As matrizes de transmissibilidade propostas neste trabalho podem ser objeto de outras aplicações, tais como: a detecção e quantificação de danos estruturais, a atualização de modelos de elementos finitos e a identificação de forças dinâmicas.

- A ferramenta computacional Myfem Modal 2D para a análise modal teórica e de dados de resposta de um modelo estrutural no plano proposta pelo autor desta 
pesquisa, a qual é apresentada no apêndice deste trabalho é também motivo para o desenvolvimento de futuros trabalhos na procura de aperfeiçoar o uso dessa ferramenta. Ditos trabalhos podem ser, entre outros: realizar uma extensão do programa para a análise de estruturas tipo pórtico em 3d e estruturas planares, implementar outras metodologias de identificação modal principalmente no domínio do tempo e desenvolver ferramentas gráficas para a análise de resultados é a visualização dos dados de entrada. 



\section{REFERÊNCIAS}

ALLEMANG, R. J. The modal assurance criterion-twenty years of use and abuse. Sound and Vibration, v. 37, n. 8, p. 14-23, 2003.

ANDERSEN, P. et al. Comparison of system identification methods using ambient bridge test data. Proceedings 17st IMAC., 1999, Kissimee, Florida.

ARAUJO, I.; MALDONADO, E.; CHO, G. Ambient vibration testing and updating of the finite element model of a simply supported beam bridge. Frontiers of Architecture and Civil Engineering in China, v. 5, n. 3, p. 344-354, 2011. ISSN 1673-7407. Disponível em: $<$ http://dx.doi.org/10.1007/s11709-011-0124-8 >.

ARAÚJO, I. G.; LAIER, J. E. Operational modal analysis using SVD of power spectral density transmissibility matrices. Mechanical Systems and Signal Processing, v. 46, n. 1, p. 129145, 2014.2 Disponível em: < http://www.sciencedirect.com/science/article/pii/S088832701400034X >.

ARTEMIS. Ambient Response Testing and Modal Identification Software. Alborg, Denmark: Structural Vibration Solution A/S 2010.

ASMUSSEN, J.; IBRAHIM, S.; BRINCKER, R. Random decrement: identification of structures subjected to ambient excitation. Proceedings 16st IMAC, 1998, Santa Barbara, California. p.914-921.

ASMUSSEN, J. C. Modal analysis based on the random decrement technique: application to civil engineering structures. 1997. (Ph.D). Denmark,University of Aalborg

ASMUSSEN, J. C.; IBRAHIM, S.; BRINCKER, R. Random decrement: identification of structures subjected to ambient excitation. Proceedings 16st IMAC, 1998, Santa Barbara, California. p.914-921.

BLACKMAN, R. B.; TUKEY, J. W. The measurement of power spectra. Dove, Publications Inc., New York, 1958.

BRINCKER, R.; KIRKEGAARD, P. H. Special issue on Operational Modal Analysis. Mechanical Systems and Signal Processing, v. 24, n. 5, p. 1209-1212, Jul 2010. ISSN 0888-3270. Disponível em: <<Go to ISI>://WOS:000279131000001 >.

BRINCKER, R. et al. Identification of dynamical properties from correlation function estimates: Danish Society for Structural Science and Engineering 1992. 
BRINCKER, R.; VENTURA, C.; ANDERSEN, P. Damping estimation by frequency domain decomposition. 19th International Modal Analysis Conference, 2001. p.698-703.

BRINCKER, R.; ZHANG, L.; ANDERSEN, P. Modal identification from ambient responses using frequency domain decomposition. Proceedings 18st IMAC, 2000, San Antonio, Texas. p.625-630.

BROWNJOHN, J. M. W. et al. Ambient vibration survey of the Bosporus suspension bridge. Earthquake engineering \& structural dynamics, v. 18, n. 2, p. 263-283, 1989. ISSN 10969845.

CAUBERGHE, B. Applied frequency-domain system identification in the field of experimentaland operational modal analysis. 2004. (Ph.D Thesis). Vrije Universiteit Brussel, Belgium.

CAUGHEY, T. K. Classical Normal Modes in Damped Linear Dynamic Systems. Journal of Applied Mechanics, v. 27, n. 2, p. 269-271, 1960. Disponível em: $<\quad$ http://link.aip.org/link/?AMJ/27/269/1 >.Disponível em: $<$ http://dx.doi.org/10.1115/1.3643949 $>$.

CHOPRA, A. K. Dynamics of structures: Theory and applications to earthquake engineering. Prentice-Hall International Series in Civil Engineering and Engineering Mechanics, 2001.

COOLEY, J. W.; TUKEY, J. W. An algorithm for the machine calculation of complex Fourier series. Mathematics of computation, p. 297-301, 1965. ISSN 0025-5718.

DEVRIENDT, C.; DE SITTER, G.; GUILLAUME, P. An operational modal analysis approach based on parametrically identified multivariable transmissibilities. Mechanical Systems and Signal Processing, v. 24, n. 5, p. 1250-1259, Jul 2010. ISSN 0888-3270. Disponível em: < $<$ Go to ISI >://WOS:000279131000005 >.

DEVRIENDT, C.; GUILLAUME, P. The use of transmissibility measurements in output-only modal analysis. Mechanical Systems and Signal Processing, v. 21, n. 7, p. 2689-2696, Oct 2007. ISSN 0888-3270. Disponível em: <<Go to ISI>://WOS:000249611900001 >.

Identification of modal parameters from transmissibility measurements. Journal of Sound and Vibration, v. 314, n. 1-2, p. 343-356, Jul 8 2008. ISSN 0022-460X. Disponível em: $<<$ Go to ISI : :/MOS:000256195100021 >.

DEVRIENDT, C. et al. Combining multiple single-reference transmissibility functions in a unique matrix formulation for operational modal analysis. Mechanical Systems and Signal Processing, v. 40, n. 1, p. 278-287, Oct 2013. ISSN 0888-3270. Disponível em: < <Go to ISI ://WOS:000323803100017 >. 
DOEBLING, S. W. et al. Damage identification and health monitoring of structural and mechanical systems from changes in their vibration characteristics: a literature review. Los Alamos National Lab., NM (United States). 1996

EWINS, D. J. Modal testing: theory, practice and application. Research studies press Baldock, 2000.

FELBER, A. J. Development of a hybrid bridge evaluation system. 1993. (Ph.D). Department of Civil Engineering,, University of British Columbia,, Vancouver, Canada,.

FONTUL, M. et al. Transmissibility matrix in harmonic and random processes. Shock and Vibration, v. 11, n. 5-6, p. 563-571, 2004 2004. ISSN 1070-9622. Disponível em: < <Go to ISI>://WOS:000226502800004 >.

FUKUZONO, K. Investigation of multiple-reference Ibrahim time domain modal parameter estimation technique. 1986. (M.Sc). University of Cincinnati

GENTILE, C.; SAISI, A. Ambient vibration testing and condition assessment of the Paderno iron arch bridge (1889). Construction and Building Materials, v. 25, n. 9, p. 3709-3720, Sep 2011. ISSN 0950-0618. Disponível em: < <Go to ISI>://WOS:000291780400007 >.

GUILLAUME, P.; HERMANS, L.; VAN DER AUWERAER, H. Maximum Likelihood Identification of Modal Parameters from Operational Data. Proceedings of IMAC 17, International Modal AnalysisConference, 1999, Kissimmee, Florida, USA.

HAMTAEI, M. R.; ANVAR, S. A. Estimation of modal parameters of buildings by Wavelet Transform. The 14th World Conference on Earthquake Engineering, 2008, Beijing, China.

HAN, J.; LI, D.; LI, H. Application of Hilbert-Huang transform and stochastic subspace identification for modal parameter identification of civil engineering structures. 2007. 216-221 ISBN 978-7-5641-0958-5. Disponível em: < <Go to ISI>://WOS:000251357300027 $>$.

HANSEN, $P$. The truncatedSVD as a method for regularization. BIT Numerical Mathematics, v. 27 , n. 4, p. 534-553, 1987. Disponível em: $<\underline{\text { http://dx.doi.org/10.1007/BF01937276 }}>$.

HAYES, M. H. Statistical digital signal processing and modeling. John Wiley \& Sons, 1996. ISBN 8126516100. 
$\mathrm{HE}, \mathrm{X} . \mathrm{H}$. et al. EMD-based random decrement technique for modal parameter identification of an existing railway bridge. Engineering Structures, v. 33, n. 4, p. 1348-1356, Apr 2011. ISSN 0141-0296. Disponível em: <<Go to ISI>://WOS:000289026900028 >.

HOA, L. T. et al. Output-only system identification using Wavelet transform. Internacional Conference on Engineering Mechanics and Automation (ICEMA 2010). Hanoi, Vietnam 2010.

HUANG, N. E. et al. The empirical mode decomposition and the Hilbert spectrum for nonlinear and non-stationary time series analysis. Proceedings of the Royal Society of London. Series A: Mathematical, Physical and Engineering Sciences, v. 454, n. 1971, p. 903-995, 1998. ISSN 1364-5021.

IBRAHIM, S. R. Efficient random decrement computation for identification of ambient responses. Proceedings of SPIE, the International Society for Optical Engineering, 2001, Society of Photo-Optical Instrumentation Engineers. p.1-6.

JAMES, G. H.; CARNE, T. G.; LAUFFER, J. P. The natural excitation technique (NExT) for modal parameter extraction from operating wind turbines. Sandia National Labs., Albuquerque, NM (United States). 1993

JUANG, J. N.; PAPPA, R. S. An eigensystem realization algorithm for modal parameter identification and model reduction. Journal of Guidance Control, and Dynamics,, v. 8, n. 5, p. 620-627, 1985.

KAY, S. M. Intuitive probability and random processes using Matlab. Springer, 2006. ISBN 0387241574.

LARDIES, J.; TA, M. N.; BERTHILLIER, M. Modal parameter estimation based on the wavelet transform of output data. Archive of Applied Mechanics, v. 73, n. 9-10, p. 718-733, Apr 2004. ISSN 0939-1533. Disponível em: <<Go to ISI>://WOS:000221055200010 >.

LE, T.-P.; PAULTRE, P. Modal identification based on the time-frequency domain decomposition of unknown-input dynamic tests. n. Accepted Manuscript, 2013. Disponível em: < http://www.sciencedirect.com/science/article/pii/S0020740313000805 >.

LE, T. H.; TAMURA, Y. Modal identification of ambient vibration structure using frequency domain decomposition and wavelet transform. The Seventh Asia-Pacific Conference on Wind Engineering, 2009, Taipei, Taiwan.

LECLERE, Q.; ROOZEN, N. B.; SANDIER, C. On the use of the H-s estimator for the experimental assessment of transmissibility matrices. Mechanical Systems and Signal Processing, v. 43, n. 1-2, p. 237-245, Feb 3 2014. ISSN 0888-3270. Disponível em: < <Go to ISI>://WOS:000329891700015 >. 
LIU, J. et al. Modal parameters identification of offshore platform using HHT method. Proceedings of the International Offshore and Polar Engineering Conference, 2012. p.11131118.

LIU, W. J. et al. Transmissibility properties of MDOF systems. 16th International Modal Analysis Conference (IMAC) - Model Updating and Correlation, 1998, Santa Barbara, Ca. Feb 02-05. p.847-854.

LIU, Y. C.; LOH, C. H.; NI, Y. Q. Stochastic subspace identification for output-only modal analysis: Application to super high-rise tower under abnormal loading condition. Earthquake Engineering and Structural Dynamics, v. 42, n. 4, p. 477-498, 2013. Disponível em: $<\quad$ http://www.scopus.com/inward/record.url?eid=2-s2.084874779839\&partnerID=40\&md5=5d67f52db20b7a930e0b2fb59f0ccf71 > .

MACEC. A Matlab Toolbox for Experimental and Operational Modal Analysis. Leuven, Belgium: Department of Civil Engineering, Faculty of Engineering, Katholieke Universiteit 2011.

MAGALHAES, F.; CUNHA, A.; CAETANO, E. Vibration based structural health monitoring of an arch bridge: From automated OMA to damage detection. Mechanical Systems and Signal Processing, v. 28, p. 212-228, Apr 2012. ISSN 0888-3270. Disponível em: < <Go to ISI : //WOS:000301549900015 >.

MAGALHÃES, F. Operational modal analysis for testing and monitoring of bridges and special structures. 2013. (Ph.D). Faculty of Engineering, University of Porto, Porto, Portugal.

MAGALHÃES, F.; CUNHA, Á. Explaining operational modal analysis with data from an arch bridge. Mechanical Systems and Signal Processing, v. 25, n. 5, p. 1431-1450, 2011. ISSN 0888-3270.

OPPENHEIM, A. V.; SCHAFE, R. W. Discrete-Time Signal Processing. Prentice-Hall, 1989.

OVERSCHEE, P. V. et al. Subspace Identification for the Linear Systems: TheoryImplementation. Kluwer academic publishers, 1996.

PEETERS, B.; DE ROECK, G. Stochastic system identification for operational modal analysis: A review. Journal of Dynamic Systems Measurement and ControlTransactions of the Asme, v. 123, n. 4, p. 659-667, Dec 2001. ISSN 0022-0434. Disponível em: $<<$ Go to ISI $>$ :/MOS:000172894900014 >. 
PEETERS, B.; VAN DER AUWERAER, H. PolyMax: a Revolution in Operational Modal Analysis. Proceedings of IOMAC, International Operational Modal Analysis Conference, 2005, Copenhagen,Denmark.

PEETERS, B. et al. The PolyMAX frequency-domain method: a new standard for modal parameter estimation? Shock and Vibration, v. 11, n. 3-4, p. 395-409, 2004 2004. ISSN 1070-9622. Disponível em: <<Go to ISI>://WOS:000224307700020 >.

REYNDERS, E. System identification methods for (operational) modal analysis: review and comparison. Archives of computational methods in engineering, v. 19, n. 1, 2012.

RIBERIO, A. M. R.; SILVA, J. M. M.; MAIA, N. M. M. On the generalization of the transmissibility concept. Mechanical Systems and Signal Processing, v. 14, n. 1, p. 29-35, 2000.

RINGWOOD, J. Multivariable control using the singular value decomposition in steel rolling with quantitative robustness assessment. v. 3, n. 4, p. 495-503, 1995. Disponível em: < http://www.sciencedirect.com/science/article/pii/096706619500021L >.

RODRIGUES, J.; BRINCKER, R. Application of the Random Decrement Technique in Operational Modal Analysis. Proceedings 1st IOMAC Conference, 2005, Copenhagen, Denmark.

SDTOOLS. Structural Dynamics Toolbox. Paris, France: SDTools Company 2008.

STRUM, R. D.; KIRK, D. E. Contemporary linear systems using MATLAB. Brooks/Cole Publishing Co., 1999. ISBN 0534371728.

UHL, T.; KLEPKA, A. Application of wavelet transform to identification of modal parameters of nonstationary systems. Journal of theoretical and applied mechanics, v. 43, n. 2, p. 277-296, 2005.

VAROTO, P. S.; MCCONNELL, K. G. Single point vs. multi point acceleration transmissibility concepts in vibration testing. Society for Experimental Mechanics, Inc, 16 th International Modal Analysis Conference., 1998. p.83-90.

VOLD, H. et al. A multi-input modal estimation algorithm for mini-computers. SAE, 1982.

WEIJTJENS, W. et al. Operational modal parameter estimation of MIMO systems using transmissibility functions. Automatica, v. 50, n. 2, p. 559-564, Feb 2014. ISSN 0005-1098; 1873-2836. Disponível em: <<Go to ISI>://WOS:000332816400025 >. 
Dealing with periodical loads and harmonics in operational modal analysis using time-varying transmissibility functions. Mechanical Systems and Signal Processing, v. 49, n. 1-2, p. 154-164, Dec 20 2014. ISSN 0888-3270. Disponível em: < <Go to ISI>://WOS:000338809100013 >.

WELCH, P. The use of fast Fourier transform for the estimation of power spectra: a method based on time averaging over short, modified periodograms. Audio and Electroacoustics, IEEE Transactions on, v. 15, n. 2, p. 70-73, 1967. ISSN 0018-9278.

YAN, W.; REN, W. On the Use of Continuous Wavelet Transmissibility for Structural Operational Modal Analysis. Journal of Structural Engineering, n. Accepted Manuscript, 2013. Disponível em: < http://ascelibrary.org/doi/abs/10.1061/\%28ASCE\%29ST.1943$\underline{541 X .0000711>\text {. }}$

YAN, W.-J.; REN, W.-X. Operational Modal Parameter Identification from Power Spectrum Density Transmissibility. Computer-Aided Civil and Infrastructure Engineering, v. 27, n. 3, p. 202-217, Mar 2012. ISSN 1093-9687. Disponível em: < <Go to ISI>://WOS:000299929700004 >.

YE, Q. W.; FENG, Z. M.; HU, H. G. An Improved Random Decrement Algorithm and Applications. Applied Mechanics and Materials, v. 121, p. 4372-4376, 2012. ISSN 3037852828.

YU, D. H.; REN, W. X. EMD-based stochastic subspace identification of structures from operational vibration measurements. Engineering Structures, v. 27, n. 12, p. 1741-1751, Oct 2005. ISSN 0141-0296. Disponível em: <<Go to ISI>://WOS:000231892600004 >.

ZHANG, L.; BRINCKER., R.; ANDERSEN, P. An overview of operational modal analysis: major development and issues. Proceedings 1st IOMAC, 2005, Copenhagen, Denmark.

ZHANG, L.; WANG, T.; TAMURA, Y. A frequency-spatial domain decomposition (FSDD) method for operational modal analysis. Mechanical Systems and Signal Processing, v. 24, n. 5, p. 1227-1239, Jul 2010. ISSN 0888-3270. Disponível em: $<<$ Go to ISI ://MOS:000279131000003 >. 



\title{
APÊNDICE A: FERRAMENTA COMPUTACIONAL PARA A ANÁLISE MODAL, MYFEM MODAL $2 D$.
}

\begin{abstract}
Embora, algumas das mais relevantes bases teóricas para a análise modal operacional foram introduzidas no Capítulo 2 e 3, é importante estabelecer como implementar e aplicar essas teorias em modelos estruturais complexos, assim como também com o intuito de simular nesses modelos dados experimentais para validação dos métodos de identificação modal. Dessa forma, ter ao alcance a possibilidade de combinar em uma mesma ferramenta computacional análise modal teórico, simulação de dados e identificação modal experimental.
\end{abstract}

Nesse contexto vários programas comerciais têm sido desenvolvidos para a análise modal teórico com elementos finitos, análise modal experimental e a combinação deles, por exemplo, para último caso temos a ferramenta computacional Structural Dynamics Toolbox SDTools (2008) feita no ambiente de Matlab. No entanto, está ferramenta aborda só métodos de identificação para a análise modal tradicional (entrada-saída) e não é disponível para a análise modal operacional. Outros tipos de ferramentas computacionais exclusivamente feitas para a análise modal experimental e não em combinação com análise modal teórico, são MACEC (2011) em ambiente de Matlab, para a análise modal experimental tradicional e operacional, e a ferramenta Ambient Response Testing and Modal Identification Software ARTeMIS (2010) só para a análise modal operacional. Porém, estas ferramentas computacionais desenvolvidas têm algumas limitações para um âmbito de pesquisa, já que não é possível ter acesso aos algoritmos usados para a análise, além da impossibilidade de poder desenvolver futuras aplicações dentro delas, no âmbito dos métodos de identificação modal.

Além disso, existe a necessidade para o desenvolvimento de este trabalho combinar análise modal experimental com o teórico. Isso permitirá validar a implementação dos algoritmos de identificação modal através da comparação com as características dinâmicas teóricas do modelo estrutural. Nesse procedimento, precisa-se de uma simulação de dados experimentais saídos do mesmo modelo estrutural, para assim criar dados similares aos que se poderiam obter em uma medição de vibrações real. A possibilidade de simular dados experimentais para validar os métodos de identificação modal permite também simular variados tipos de condições na identificação modal, tais como os tipos de excitação (ruído branco ou ruído colorido), presença de ruídos no sinal de saída ou identificação com frequências de ressonância muito próximas, entre outras. Evidentemente, esses tipos de 
simulações não substituem os dados experimentais reais, já que em eles podem existir incertezas que não se conseguem simular numericamente.

Neste trabalho uma nova ferramenta computacional para a análise modal teórico e análise modal operacional tem sido desenvolvida no ambiente de Matlab para a análise de estruturas tipo pórtico no plano. O modelo estrutural é conformado a partir da teoria de elementos finitos, na qual se podem fazer: análise estática, análise de instabilidade para pequenos deslocamentos, análise de modos de flambagem, análise sob carregamento dinâmico, análise modal teórico e análise modal operacional.

Para a análise modal teórico podem-se avaliar as seguintes características do modelo estrutural: frequências de vibração, formas modais e taxas de amortecimento modal, funções de resposta em frequência (FRFs), funções de densidade espectral (PSDs), funções de transmissibilidade multivariável (FTs), funções de resposta impulsiva (FRIs) e funções de correlação da resposta (FCs).

No caso de análise modal operacional, por enquanto, são apresentadas neste apêndice as diferentes técnicas e métodos no domínio da frequência detalhados no Capítulo 3, os quais são: métodos para a estimativa das funções de correlação, como o método direto, método do periodograma médio e o método de decremento aleatório; os métodos para a estimativa das funções densidade espectrais, como o método de Welch e o método de Blackman e Tukey e os método e as técnicas de identificação modal, como a média espectral (PP), técnica SVD para a decomposição da matriz espectral, a transformação linear do espectro e o método de transmissibilidade com densidade espectral.

Por último, é importante estabelecer que a intenção do presente apêndice, é ser uma guia introdutiva ao uso da ferramenta proposta, portanto, são comentadas somente algumas das ferramentas desenvolvidas no programa e não a totalidade delas.

\section{A.1 DESCRIÇÃO GERAL DE MYFEM MODAL 2D.}

A ferramenta computacional MYFEM MODAL 2D foi desenvolvida na linguagem de programação de Matlab com orientação a objetos para o análise de estruturas tipos pórtico no plano baseado no modelamento de elementos finitos. A ferramenta contém a possibilidade de fazer a análise estática, análise instabilidade para pequenos deslocamentos, análise dinâmica e análise modal teórico e operacional.

No modelamento em elementos finitos, além da contribuição da rigidez elástica nos elementos tipo pórtico produto da deformação axial e da flexão, foi implementado no 
programa o aporte da rigidez de molas e bases elásticas, bem como o modelamento de rótulas nos nós para considerar momentos iguais a zeros.

O programa tem como variável principal o objeto chamado Fem que contém a maioria das propriedades e dos métodos para a organização (pré-processamento), análise (processamento) e apresentação de resultados (pós-processamento) do modelo estrutural. Então, no caso da obtenção da informação das características geométricas, de materiais e o carregamento do modelo estrutural conta-se com uma série de métodos, que são os seguintes: addNodes para a localização dos nós, addMat para as propriedades dos materiais, addSec para as propriedades das seções, addElem para conexão dos elementos, addRelease para a localização das rótulas, addFix para as condições de contorno, addSpringN para as molas, addSpringL para as bases elásticas, addLoadP e addLoadD para os carregamentos estáticos pontuais e distribuídos, respectivamente, e addLoadPDina para os carregamentos dinâmicos pontuais .

Do mesmo modo, na parte de processamento o objeto Fem contém os métodos que avaliam a matriz de rigidez, de massa e amortecimento, o vetor de forças estático ou dinâmico, e a vinculação das condições de contorno. Ditas matrizes e vetores são usadas para o respetivo análise, seja estático o dinâmico. Nesta parte de processamento o programa conta com outro objeto chamado Frame2D o qual contém os métodos necessários para o cálculo das matrizes de rigidez e de massa individuais de cada elemento, assim ele contém todas as funções de forma para os elementos com ou sem rótula, além de um integrador numérico para resolver as integrais pelas quais se avaliam as respectivas matrizes.

No programa existem três tipos básicos de análise da estrutura sob carregamento, um chamado de Analysis para a análise estática linear, outro chamado Pdelta para a análise não linear geométrico de pequenos deslocamentos, o qual inclui também os cálculos dos modos de Flambagem, e outro chamado de AnalysisDL para o análise dinâmica linear por meio do método integração numérica de Newmark .

No caso de análise dinâmica devem ser fornecidas informações para a avaliação da matriz de massa e amortecimento. Assim, enquanto a matriz de massa, dois tipos de matriz podem ser avaliados: matriz massa concentrada ou matriz de massa consistente. Três tipos de matrizes de amortecimento clássico são disponíveis, para a matriz de amortecimento, matriz de amortecimento de Caughey, matriz de amortecimento com amortecimentos totais e matriz de amortecimento de Rayleigh. Para a matriz de amortecimento de Rayleigh pode- 
se determinar proporcional à massa e à rigidez, só à massa ou só à rigidez. Nesse mesmo método de análise dinâmico foi implementada uma rutina para condensação estática, com o intuito de estabelecer uma redução do modelo dinâmico para alguns graus de liberdade selecionados.

Para simulações de dados experimentais, têm sido implementados no programa dois algoritmos para a geração de excitação, um para ruído branco e outro para ruído colorido. 0 algoritmo de ruído branco é baseado na geração de números aleatórios com uma distribuição gaussiana de média zero. Para o ruído colorido é gerado um ruído branco que depois é filtrado por um sistema de um só grau de liberdade. Os métodos para geração de excitação de ruído branco e ruído colorido são chamados NoiseWG e NoiseCR, respectivamente.

Na seção de pós-processamento, o objeto Fem depender do tipo de análise feito, seja estático ou dinâmico. O mesmo extrai a informação de deslocamento, dos esforços internos nos elementos e das reações nos apoios através dos métodos RecordNode ou RecordNodeDL, RecordElement ou RecordElementDL e Reactions ou ReactionsDL. Na análise dinâmica podem-se extrair além dos deslocamentos, as velocidades e acelerações da estrutura.

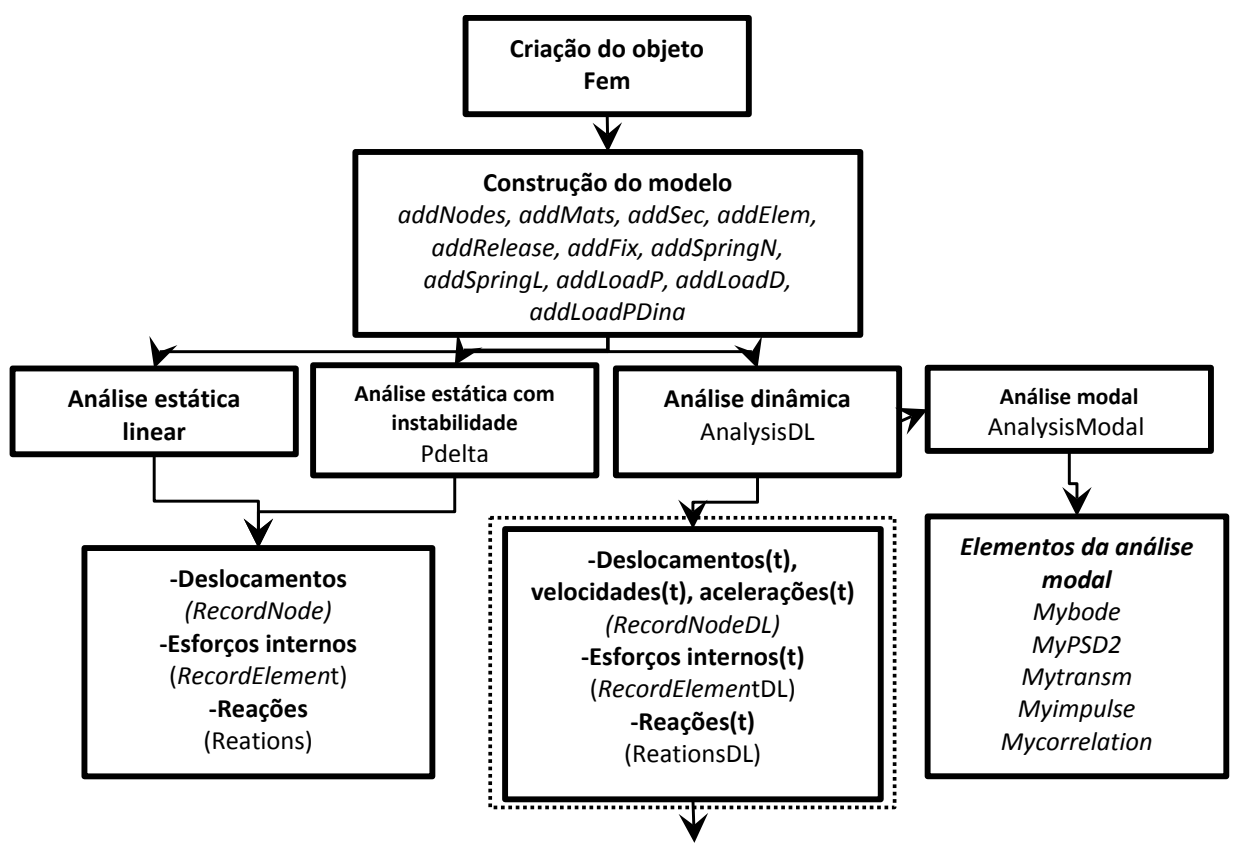

Figura A-1 Sistematização dos componentes principais do programa Myfem Modal $2 d$. 


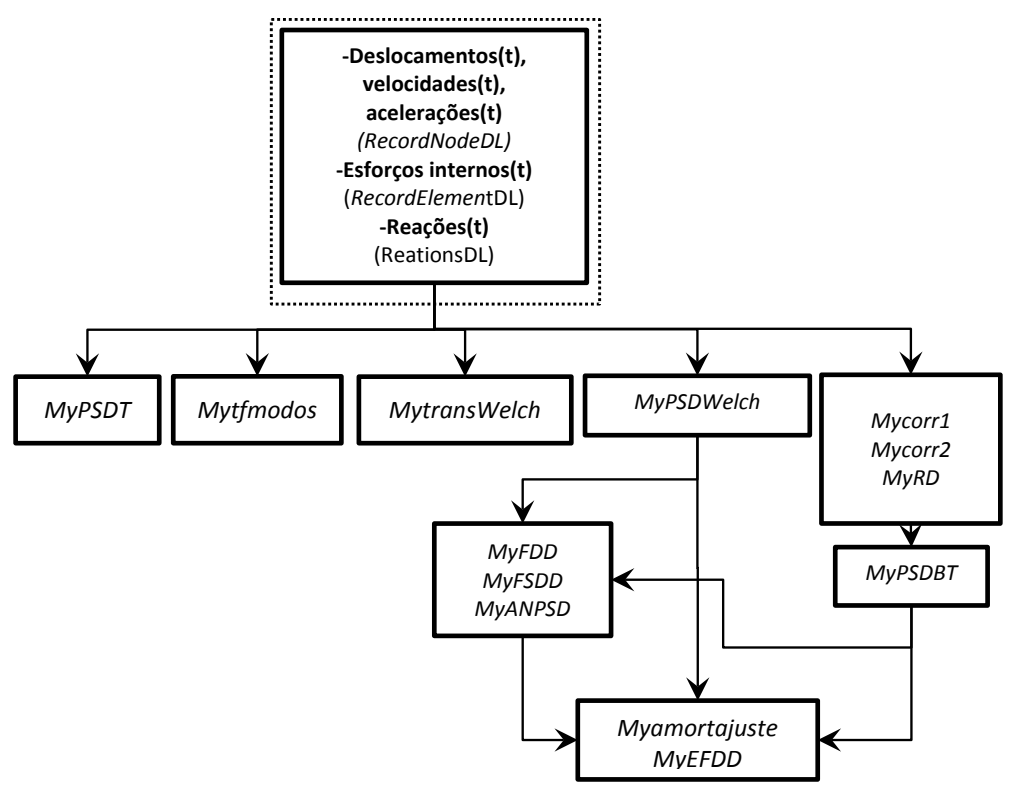

Figura A-2 Sistematização dos componentes para a análise modal a partir dados de resposta de Myfem Modal $2 d$.

Para a análise modal teórico, o programa contém os métodos: AnalysisModal para o cálculo das frequências de vibração, formas modais e taxas de amortecimento modal da estrutura, Mybode para as funções de resposta em frequência, MyPSD2 para as funções de densidade espectral, Mytransm para as funções de transmissibilidade multivariável, Mylmpulse para as funções de resposta impulsiva e MyCorrelation para as funções de correlação.

No caso da análise modal operacional em uma estrutura modelada em Myfem Modal 2d. é necessário, em primeiro lugar, realizar uma análise dinâmica da estrutura sob um carregamento determinado, assim as respostas dinâmicas nos diferentes graus de liberdade da estrutura podem ser usadas a seguir para aplicar algum tipo de metodologia de análise da resposta e obter parâmetros modais (frequências, formas modais e amortecimento modal).

O programa contém, entre outros procedimentos não comentados, diferentes ferramentas básicas de representação de dados de resposta para a análise modal, como são: Mycorr1 para a estimativa das funções de correlação pelo método direto, Mycorr2 para as funções de correlação pelo método do periodograma médio, MyRD para as funções de decremento aleatório, MyPSDWelch para as funções de densidade espectral pelo método de Welch, MyPSDBT para as funções de densidade espectral pelo método de Blackman e Tukey e MytransWelch para as funções de transmissibilidade multivariável. 
Igualmente, têm sido implementados diferentes métodos no domínio da frequência para a identificação modal como: MyANPSD para a definição da média dos auto espectros da matriz de densidade espectral, Mytfmodos para a avaliação das formas modais por meio de funções de transmissibilidade, Myamortajuste para avaliação da porcentagem de amortecimento por meio do método de ajuste do espectro analítico de um modelo de um só grau de liberdade, MyFDD para a decomposição da matriz de densidade espectral em valores singulares, MyEFDD para avaliação da porcentagem de amortecimento a partir de usar o método do decremento logarítmico à transformada inversa de Fourier de uma faixa de frequência de uma função de densidade espectral, MyFSDD para transformação linear do espectro e MyPSDT para aplicação do método de transmissibilidade com densidade espectral. Com uso do método MyFDD é possível avaliar as frequências naturais e as formas modais e com MyPSDT pode-se avaliar frequências naturais.

Finalmente, uma descrição gráfica sistematizada de alguns dos componentes do programa Myfem Modal 2d é dada na Figura A-1 e Figura A-2

\section{A.2 EXEMPLOS DE USO DO PROGRAMA PARA A ANÁLISE MODAL TEÓRICO.}

Com o intuito de se introduzir ao uso do programa mostra-se um exemplo de uma viga de $6 \mathrm{~m}$ de comprimento como é apresentado na Figura A-3, na que se visualiza a quantidade de elementos na que foi discretizada, visando conformar o modelo em elementos finitos. Igualmente, mostram-se os números dos nós, os números dos elementos e a numeração dos graus de liberdade de cada nó. Nesse modelo da viga foram usados como dados um módulo de Young de $1,781 \times 10^{10} \mathrm{~N} / \mathrm{m}^{2}$, uma densidade do material de 12816 $\mathrm{kg} / \mathrm{m}^{3}$, uma seção quadrada da viga de $0,15 \mathrm{~m} \times 0,15 \mathrm{~m}$ e valores de rigidez das molas $\mathrm{k} 1=1000 \mathrm{~N} / \mathrm{m}$ e $\mathrm{k} 2=10^{\wedge} 8 \mathrm{~N} / \mathrm{m}$.

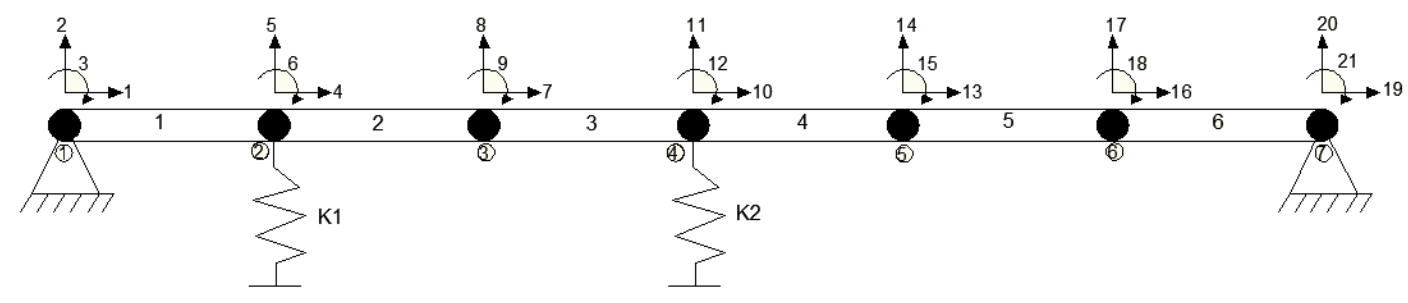

Figura A-3 Viga de exemplo para a análise modal teórico e operacional.

O primeiro passo para a análise modal da viga no programa Myfem Modal é a criação de um arquivo de extensão .m de Matlab, o qual tem que ser salvado na mesma pasta onde se encontram os arquivos do programa. Todas as pastas do programa e os arquivos dos modelos criados devem ser agregados ao Set Path ou pastas de trabalho da 
ferramenta de Matlab. Para a construção do modelo a primeira instrução é a criação do objeto Fem, como se mostra na Figura A-4.

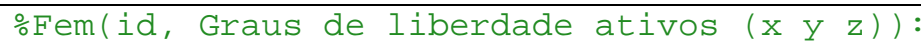

Figura A-4 Criação do objeto Fem em Myfem Modal .

$\mathrm{Na}$ anterior instrução foi criado o objeto $\mathbf{F e m}$ em uma variável chamada arbitrariamente de 'Model' com um id=1, na qual são indicados os graus de liberdade que se encontrarão ativos no modelo de duas dimensões, que são os graus de liberdade horizontal, vertical e rotacional. Nesse momento, na variável 'Model' contêm-se todas as propriedades e os métodos do objeto Fem.

O seguinte passo é a definição das características dos nós, materiais, seções, elementos, condições de contorno do modelo e localização das molas. Portanto, mostra-se a seguir uma instrução com a configuração do uso desses métodos para a criação das diferentes características do modelo com comentários que estão seguidos do símbolo '\%” para cada método (Figura A-5).

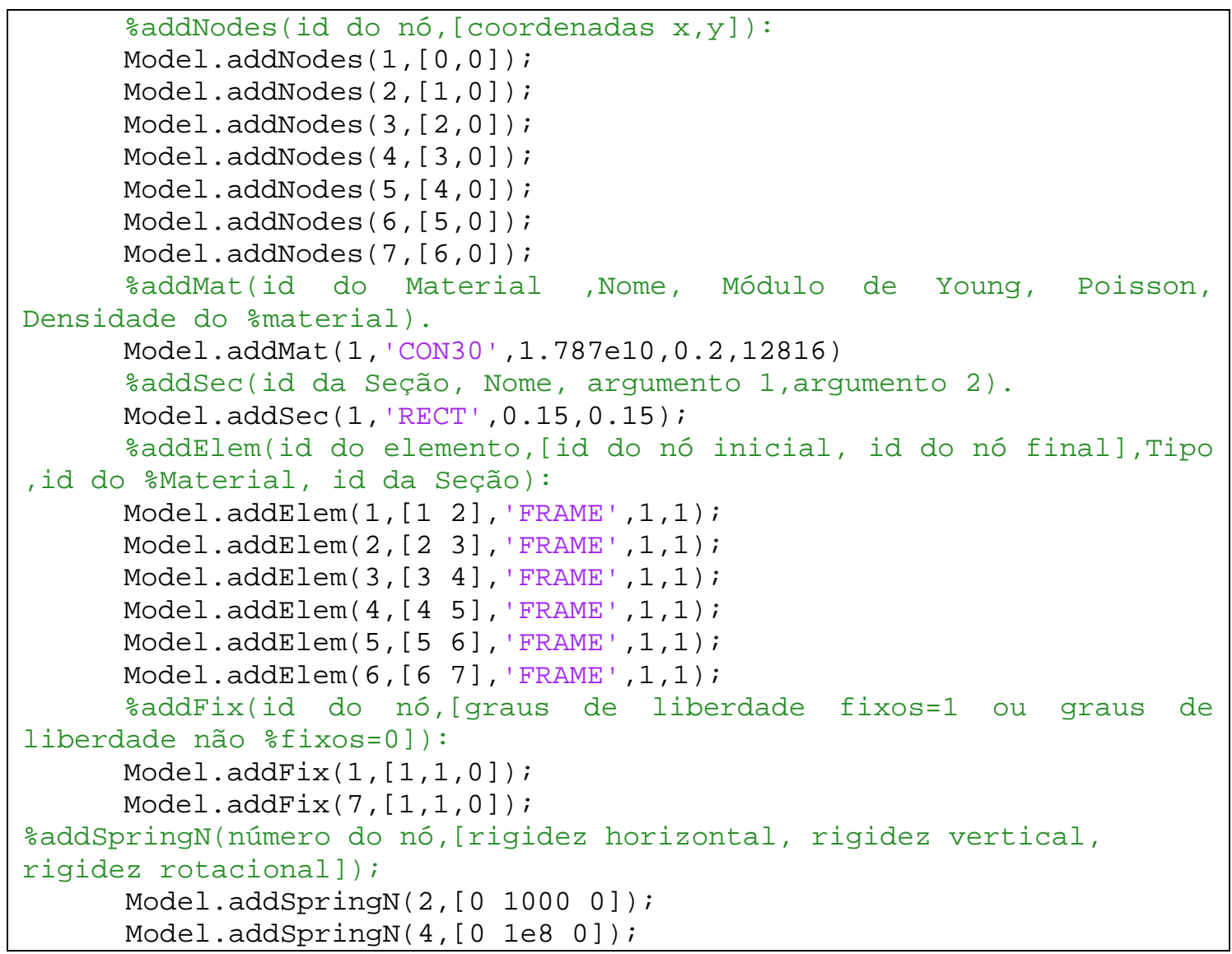

Figura A-5 Definição de características estruturais do modelo em Myfem Modal . 
O método addSec contem dois tipos de geometrias para as seções, que são tipo retangular 'RECT' e circular 'CIRC'. Então, se for tipo 'RECT', deve ser dado dois argumentos como são a largura e a altura da seção da viga. No método addElem deve-se fornecer sempre o nome de 'FRAME' para o argumento chamado 'Tipo', já que é o único tipo de elemento que contém o programa. Pode-se notar que na instrução da Figura A-5 para o uso do método addFix não se precisa colocar a informação para todos os nós, só é necessário nos nós que tiverem restrições. Os outros métodos criados para a definição de rótulas, base elástica e cargas nodais ou cargas distribuídas para a análise estática não foram usados nesse exemplo devido a que no modelo não existem essas características e não se considera uma análise estática.

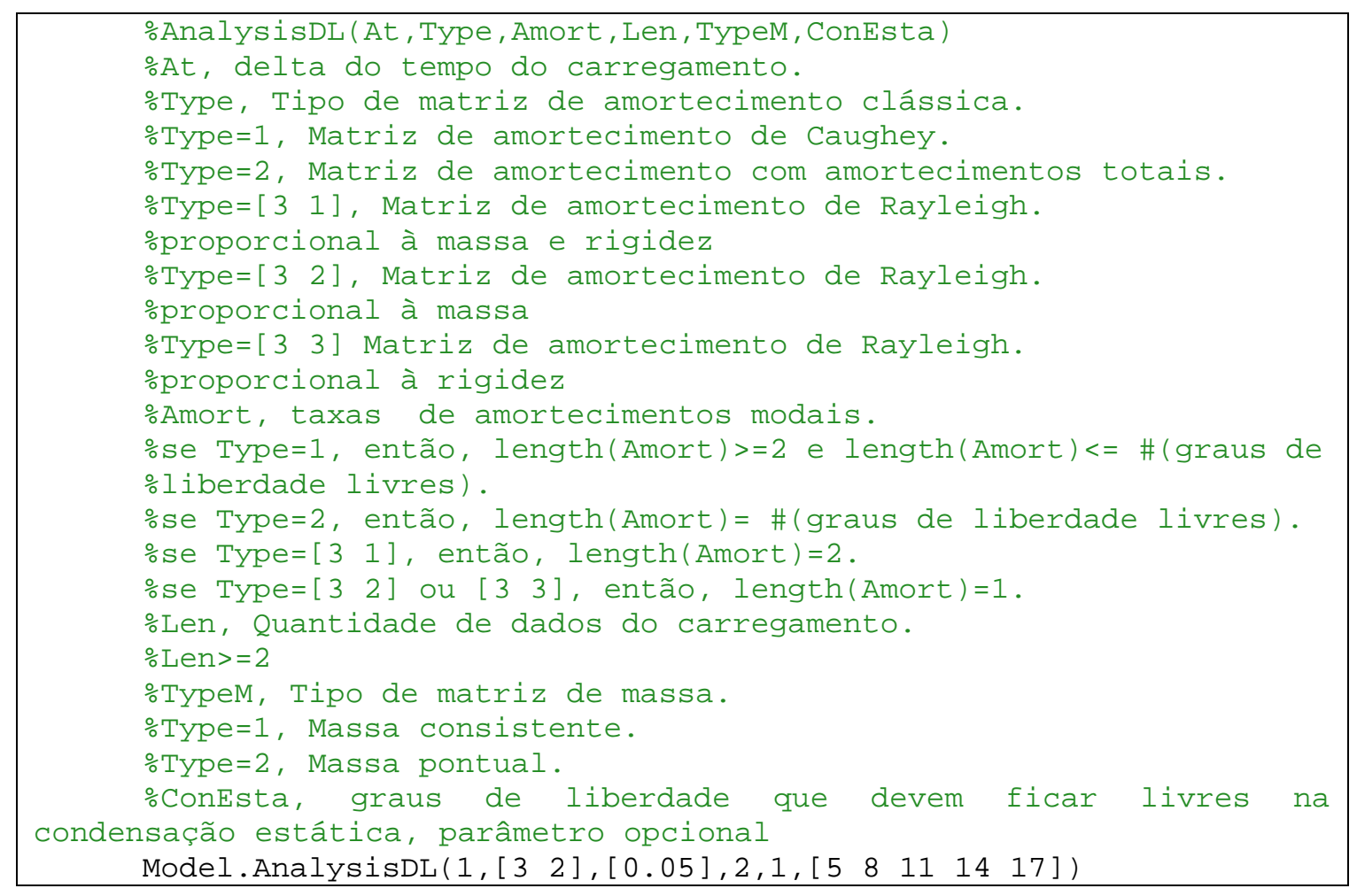

Figura A-6 Definição da análise dinâmica para a análise modal teórico em Myfem Modal .

Depois de determinar todas as características da estrutura se pode primeiro fazer uma análise dinâmica do modelo. A análise dinâmica possui internamente, além da análise de carregamento no tempo com o método de Newmark, alguns componentes da análise modal através do método AnalysisModal, os quais avaliam as frequências naturais não amortecidas e amortecidas, formas modais e taxas de amortecimento. Para isso, é necessário definir o tipo de matriz de massa e amortecimento através do método AnalysisDL. Na definição dessas matrizes o método AnalysisDL precisa dos seguintes argumentos para seu funcionamento: delta de tempo do carregamento, escolha do tipo de 
matriz de amortecimento clássica, taxas de amortecimentos modais, quantidade total de dados do carregamento, escolha do tipo de matriz de massa e um parâmetro opcional para uma condensação estática da matriz de rigidez da estrutura, que deve conter os graus de liberdade que devem ficar livres (Figura A-6).

No exemplo proposto não é considerado carregamento dinâmico, portanto, os dados para o delta de tempo e para as quantidades de dados do carregamento foram fornecidas como 1 e 2, respectivamente. Do mesmo modo, foi definida uma matriz de massa consistente e uma matriz de amortecimento clássica proporcional à massa usando uma porcentagem de amortecimento para o primeiro modo de $5 \%$. Além disso, com o fim de simplificar a quantidade de resultados para mostrar nesse exemplo, foi feita uma condensação estática do modelo, desse modo, foram selecionados só os graus de liberdade verticais como os graus de liberdade livres no modelo dinâmico, ficando com só 5 graus de liberdade.

Os resultados para as frequências naturais não amortecidas e amortecidas, taxas de amortecimentos e formas modais normalizadas à massa são recuperados da variável 'Model' da forma como se mostra na Figura A-7, as quais são reveladas na Tabela 4-1 e Tabela 4-2.

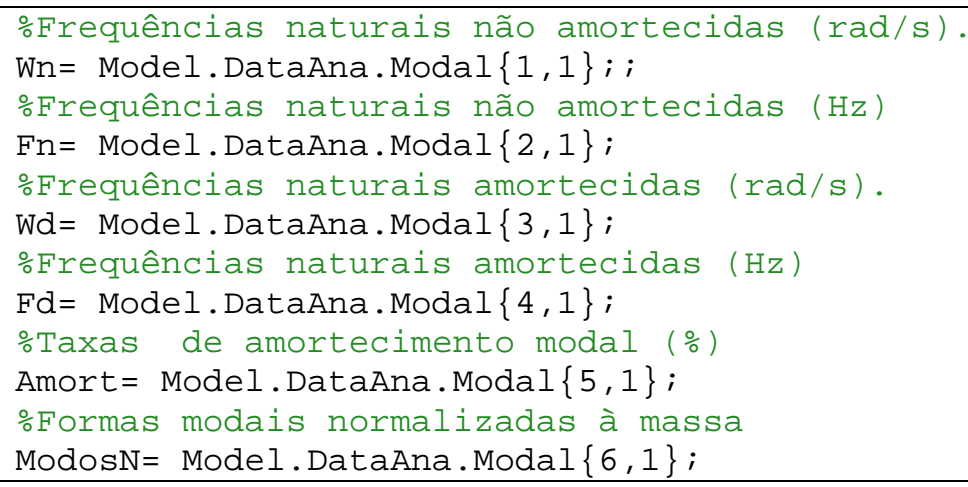

Figura A-7 Recuperação de dados de elementos da análise modal teórico em Myfem Modal .

Tabela A-1 Frequências naturais não amortecidas e amortecidas e taxas de amortecimentos do modelo de exemplo com o uso de Myfem Modal .

\begin{tabular}{ccccc}
\hline $\mathrm{Wn}(\mathrm{rad} / \mathrm{s})$ & $\mathrm{Fn}(\mathrm{Hz})$ & $\mathrm{Wd}(\mathrm{rad} / \mathrm{s})$ & $\mathrm{Fd}(\mathrm{Hz})$ & $\xi(\%)$ \\
\hline 60,010 & 9,5508 & 59,935 & 9,539 & $5,00 \%$ \\
91,882 & 14,6234 & 91,833 & 14,616 & $3,27 \%$ \\
276,784 & 44,0516 & 276,768 & 44,049 & $1,08 \%$ \\
306,005 & 48,7022 & 305,990 & 48,700 & $0,98 \%$ \\
775,889 & 123,4866 & 775,883 & 123,486 & $0,39 \%$ \\
\hline
\end{tabular}


Tabela A-2 Modos de vibração do exemplo com o uso de Myfem Modal .

\begin{tabular}{cccccc}
\hline GL & Modo 1 & Modo 2 & Modo 3 & Modo 4 & Modo 5 \\
\hline 5 & 0,0315 & $-0,0361$ & $-0,0376$ & $-0,0325$ & 0,0068 \\
8 & 0,0315 & $-0,0261$ & 0,0376 & 0,0384 & $-0,0201$ \\
11 & 0,0000 & $-0,0019$ & 0,0000 & 0,0120 & 0,0695 \\
14 & $-0,0315$ & $-0,0261$ & $-0,0376$ & 0,0384 & $-0,0201$ \\
17 & $-0,0316$ & $-0,0361$ & 0,0376 & $-0,0325$ & 0,0068 \\
\hline \multicolumn{7}{c}{ GL=Grau de liberdade } & & & &
\end{tabular}

Outros elementos da análise modal que oferece o programa Myfem Modal se descrevem a seguir, tais como os métodos usados para o cálculo de funções de resposta em frequência (FRFs), funções de densidade espectral (PSD), funções de transmissibilidade (FT), funções de resposta impulsiva (FRIs) e funções de correlação (FC).

\section{A.2.1 Funções de resposta em frequência (FRFs)}

O método utilizado de Myfem Modal para a avaliação das FRFs é chamado de Mybode, que conta com a possibilidade de: avaliar FRFs para deslocamento (receptância), velocidade (mobilidade) e aceleração (acelerancia), escolher determinados graus de liberdade para as FRFs, optar pelo cálculo da fase da FRF e fazer uma decomposição modal da FRF. Da mesma forma, o método Mybode faz automaticamente o gráfico das mesmas FRFs. Na Figura A-8, se faz uma descrição do uso do método Mybode.

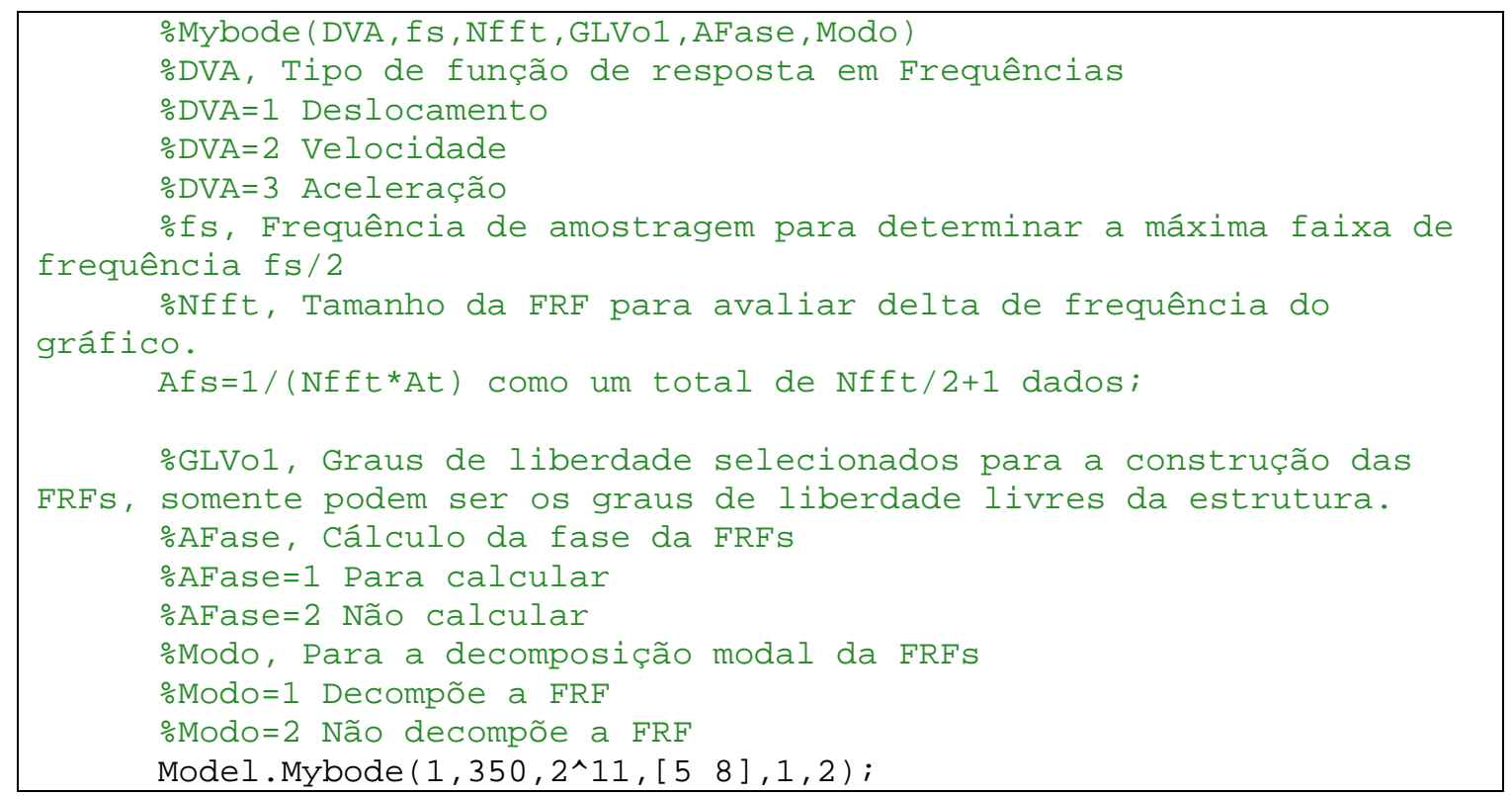

Figura A-8 Método Mybode para o cálculo das FRFs.

Como é mostrado na Figura A-8 calcula-se para o exemplo proposto com o método Mybode as FRFs de receptância para os graus de liberdade 5 e 8 até uma frequência de 
$175 \mathrm{~Hz}$, definindo um tamanho das FRFs de 1025 dados, às quais são acompanhadas do cálculo de sua fase. O resultado gerado pelo método Mybode para avaliar as FRFs nos graus de liberdade 5 e 8 é mostrado na Figura A-9.
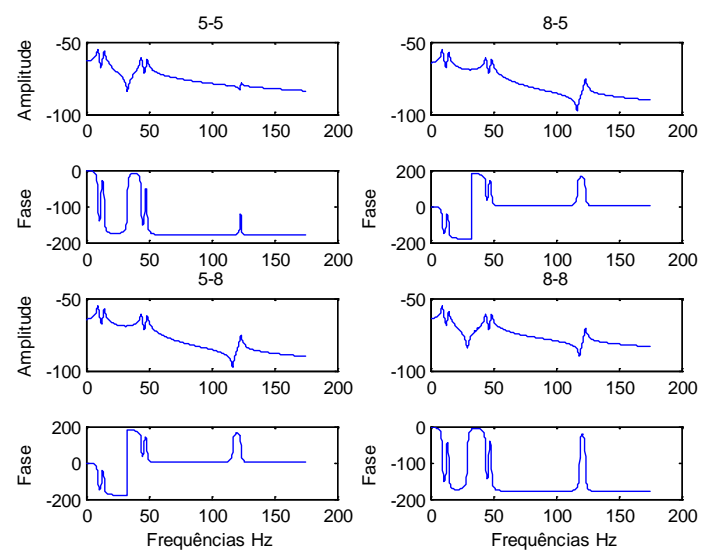

Figura A-9 FRFs teóricas para os graus de liberdade 5 e 8, calculadas com Myfem Modal .

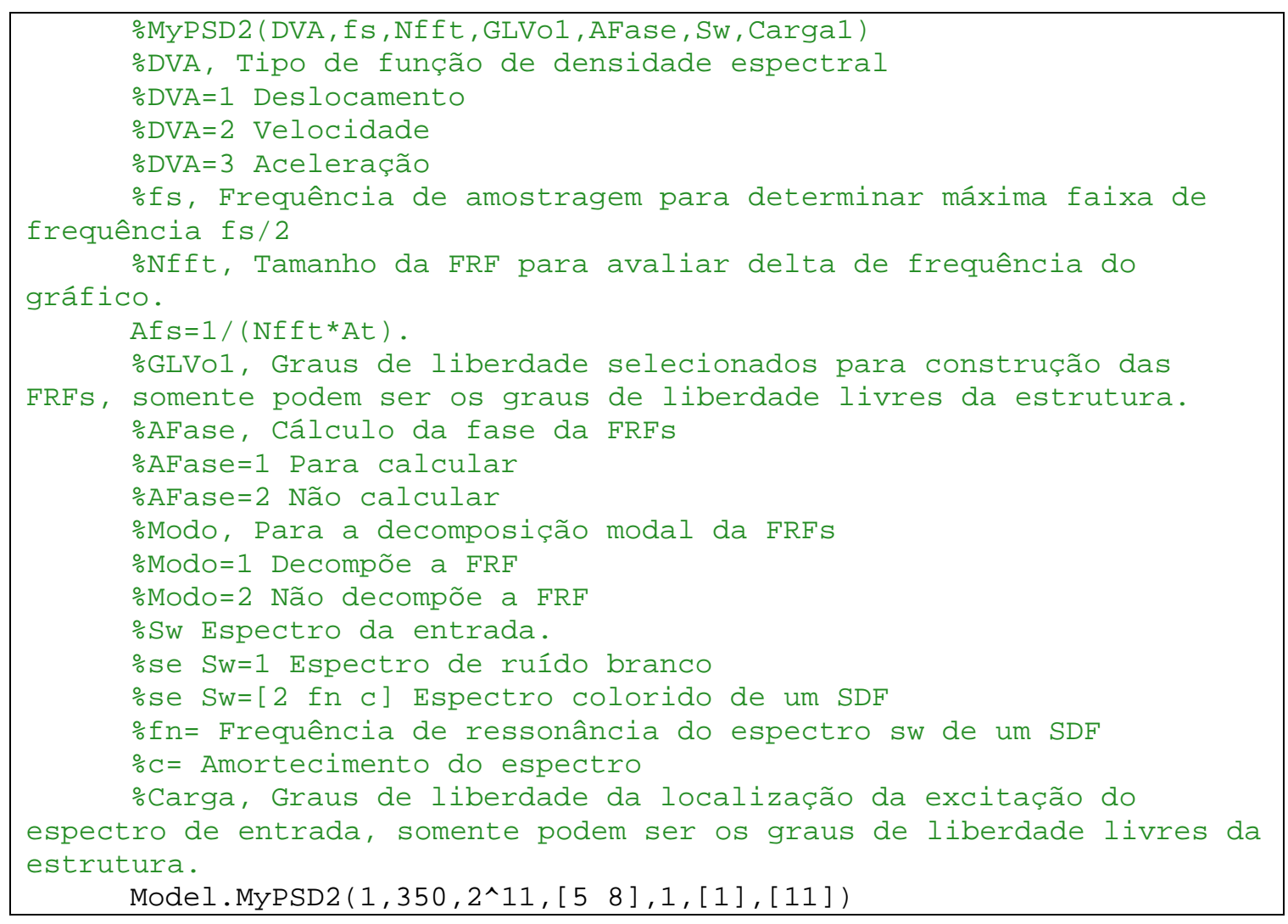

Figura A-10 Método MyPSD2 para o cálculo das PSDs. 


\section{A.2.2 Funções de densidade espectral (PSDs)}

Para a avaliação das PSDs o método de Myfem Modal é chamado MyPSD2. Nesse método é possível avaliar as PSDs para o deslocamento, velocidade e aceleração, a partir de escolher determinados graus de liberdade e selecionar o tipo de espectro de entrada, que podem ser ruído branco ou ruído colorido. Para o ruído colorido o espectro é definido como um SDF, no qual se devem determinar a frequência de ressonância e a porcentagem de amortecimento do SDF. Para o espectro de entrada pode-se determinar a localização em determinados graus de liberdade do modelo. Do mesmo modo como foi determinado para as FRFs pode-se optar pelo cálculo da fase e fazer uma decomposição modal.

Na Figura A-10 se faz uma descrição do uso do método MyPSD2 e se calculam as PSDs de deslocamento dos graus de liberdade 5 e 8 do exemplo, produto de uma excitação com espectro constante (ruído branco) localizada no grau de liberdade 11. O resultado das PSDs para essa condição de excitação é mostrada na Figura A-11, onde, pode-se notar que para uma excitação localizada no grau de liberdade 11, gera uma excitação de sós três frequências naturais da estrutura, e não das 5 que contém o modelo.
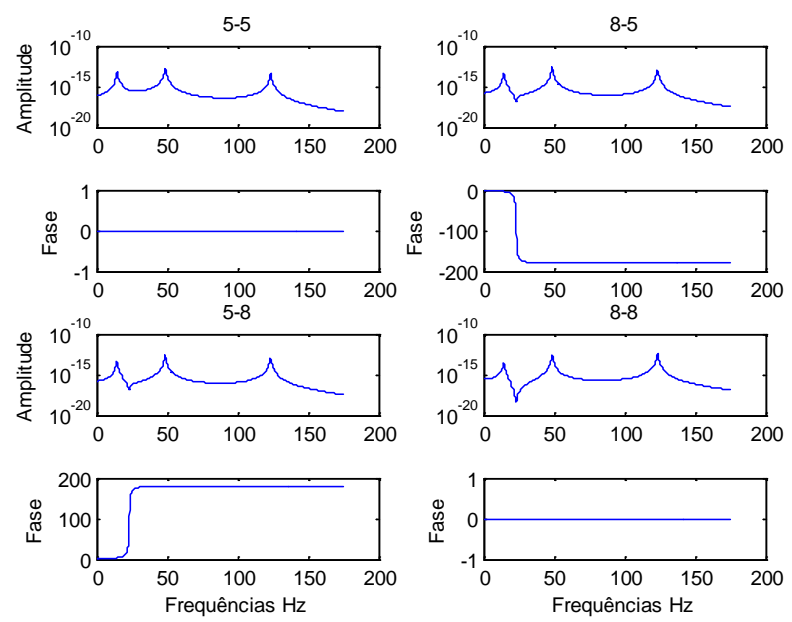

Figura A-11 PSDs teóricas para os graus de liberdade 5 e 8 para uma excitação de ruído branco localizada no grau de liberdade 11, calculadas com Myfem Modal .

\section{A.2.3 Funções de transmissibilidade multivariável (FTs)}

Para estimativa das funções de transmissibilidade o método usado de Myfem Modal é Mytransm. O método avalia as funções de transmissibilidade $T^{a}{ }_{u k}$ para múltiplos graus de liberdade, na qual, $U$ são os graus de liberdade não observados, $K$ são os graus de liberdade observados e A são os graus de liberdade da localização do carregamento. A transmissibilidade pode ser avaliada para o deslocamento, velocidade e aceleração e se pode optar pelo cálculo da fase. Na Figura A-12 se faz uma descrição do uso do método 
Mytransm e se calculam as funções de transmissibilidade entres os graus de liberdade 5, 8 e 17 com os graus de liberdade 11 e 14, localizando o carregamento nos graus de liberdade 11 e 14, do exemplo proposto. O resultado mostra-se na Figura A-13.

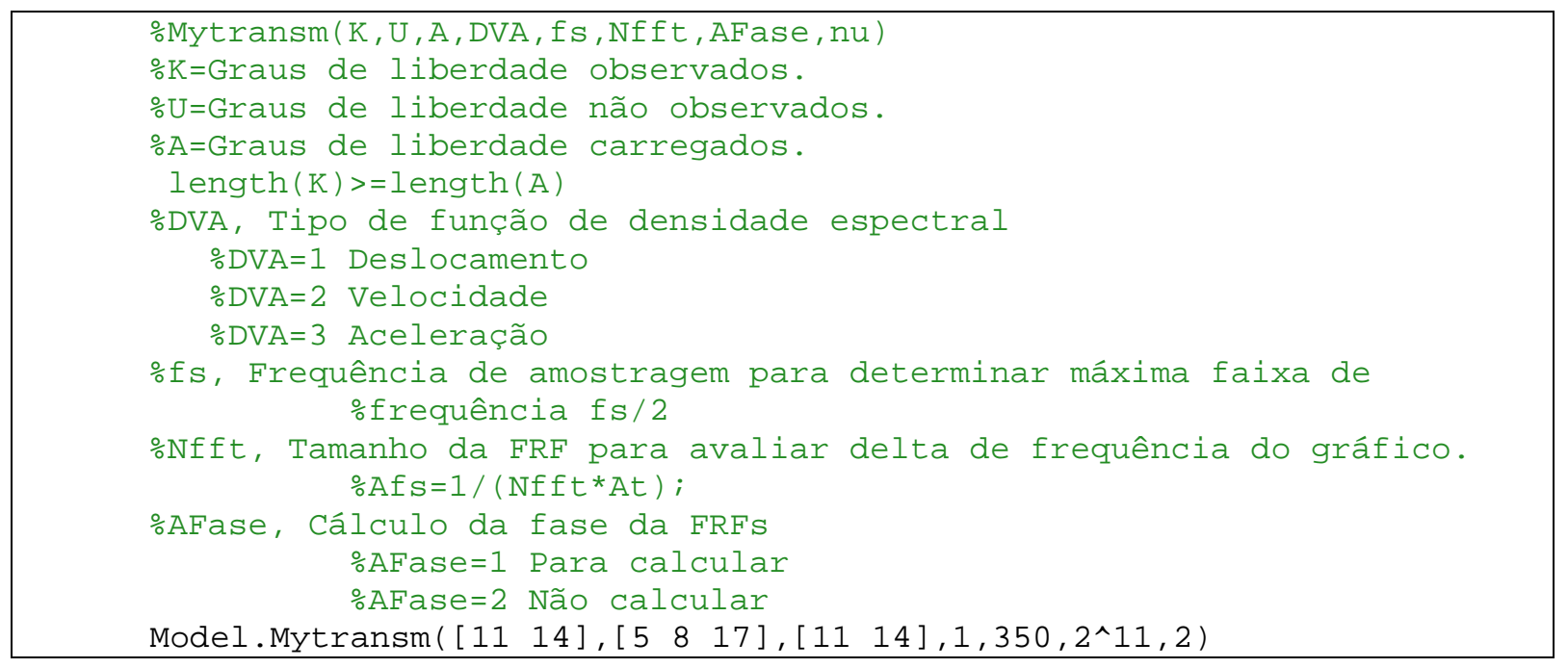

Figura A-12 Método Mytransm para o cálculo das funções de transmissibilidade em múltiplos graus de liberdade.
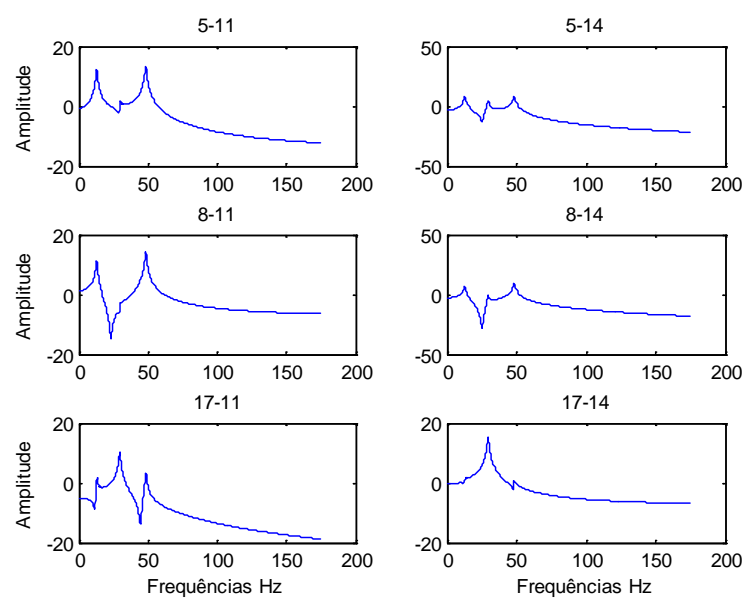

Figura A-13 FTs teóricas entre os graus de liberdade 5, 8 e 17 com 11 e 14 com carregamento em 11 e 14, calculadas com Myfem Modal .

\section{A.2.4 Funções de resposta impulsiva (FRIs)}

A resposta ao impulso em um grau de liberdade devido ao carregamento em outro, é calculado por meio do método de Myfem Modal Myimpulse, a partir de se estabelecer a 
transformada inversa de Fourier às funções de resposta em frequência. Os argumentos necessários para o funcionamento do método são: seleção do tipo de resposta ao impulso seja em deslocamento, velocidade ou aceleração, frequência de amostragem da resposta, tempo máximo da resposta, graus de liberdade onde se aplica o impulso e onde se mostra a resposta e a seleção da opção de uma decomposição modal do impulso. Na Figura A-14 mostra-se uma descrição do método Myimpulse e onde se computa a resposta impulsiva em deslocamento, nos graus de liberdade 5 e 8 com um tempo máximo de resposta de 2 segundos, o resultado é mostrado na Figura A-15.

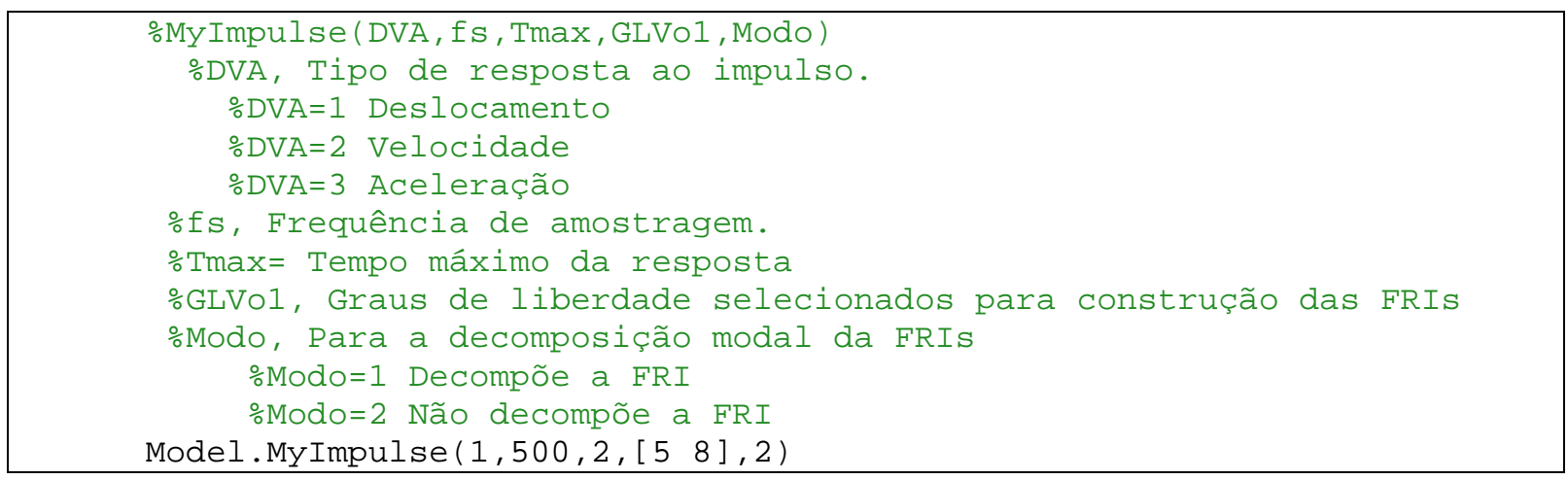

Figura A-14 Método Mylmpulse para o cálculo das funções de resposta impulsiva
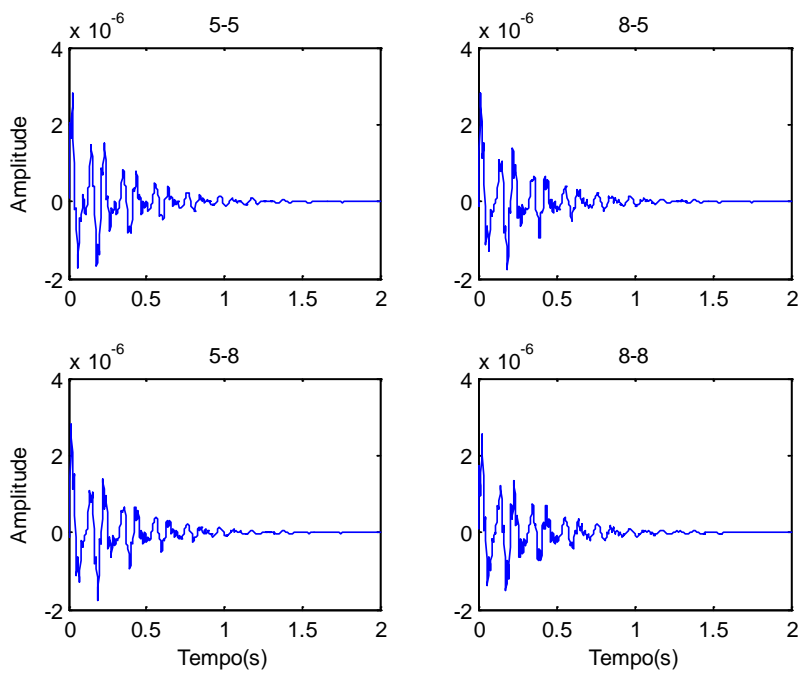

Figura A-15 FRIs teóricas nos graus de liberdade 5 e 8, calculadas com Myfem Modal

\section{A.2.5 Funções de correlação (FCs).}

As funções de correlação das respostas entre vários graus de liberdade são calculadas por meio do método desenvolvido em Myfem Modal chamado de MyCorrelation, a partir de se estabelecer a transformada inversa de Fourier das funções de densidade espectral calculadas para um determinado tipo de carregamento. O método precisa dos seguintes argumentos para seu correto funcionamento: seleção do tipo de função de 
correlação seja em deslocamento, velocidade ou aceleração, frequência de amostragem, tempo máximo da resposta, seleção dos graus de liberdade, espectro de entrada do carregamento e localização do mesmo nos graus de liberdade. Na Figura A-16 mostra-se a descrição do método MyCorrelation e computa-se as funções de correlação em deslocamento para os graus de liberdade 5 e 8 para uma entrada tipo ruído branco localizada no grau de liberdade 11.

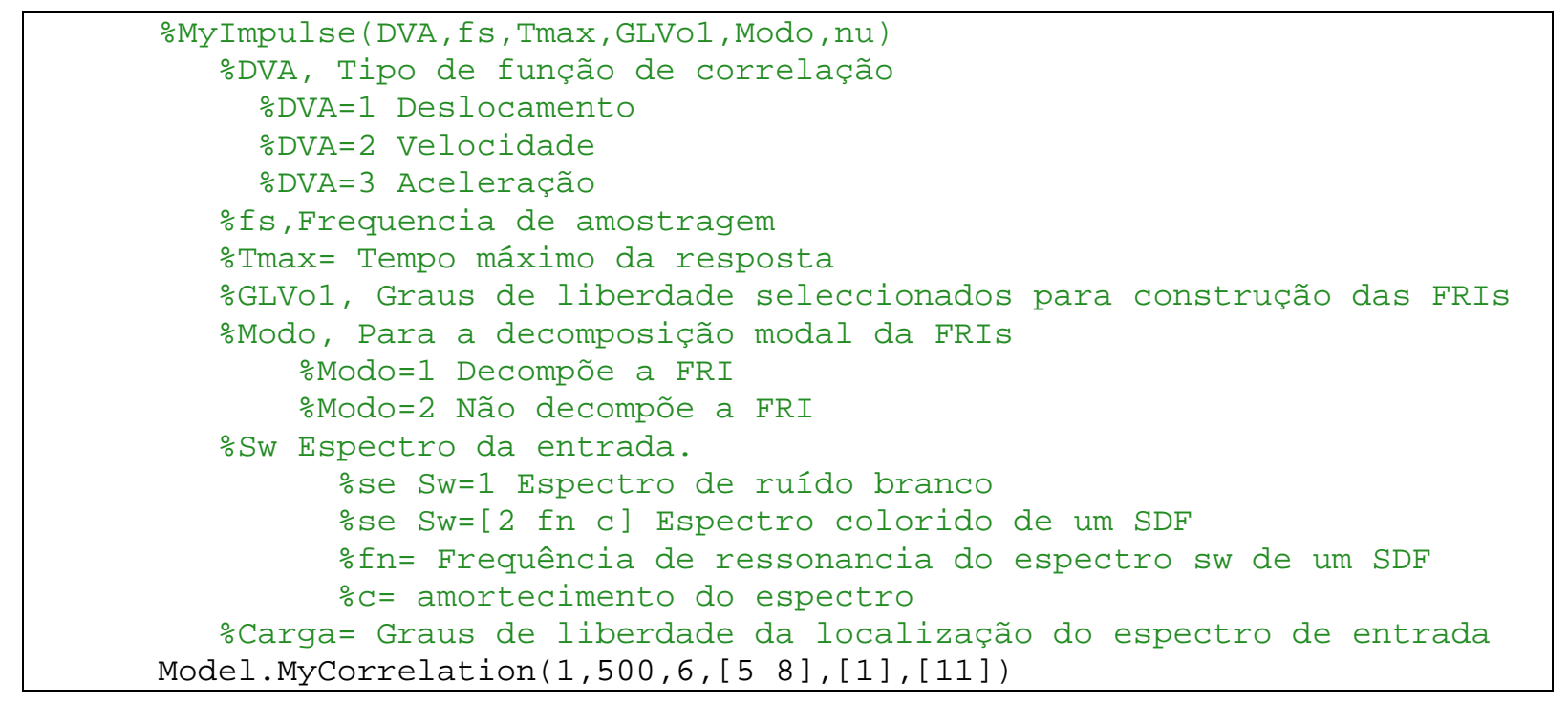

Figura A-16 Método MyCorrelation para o cálculo das funções de correlação da resposta.
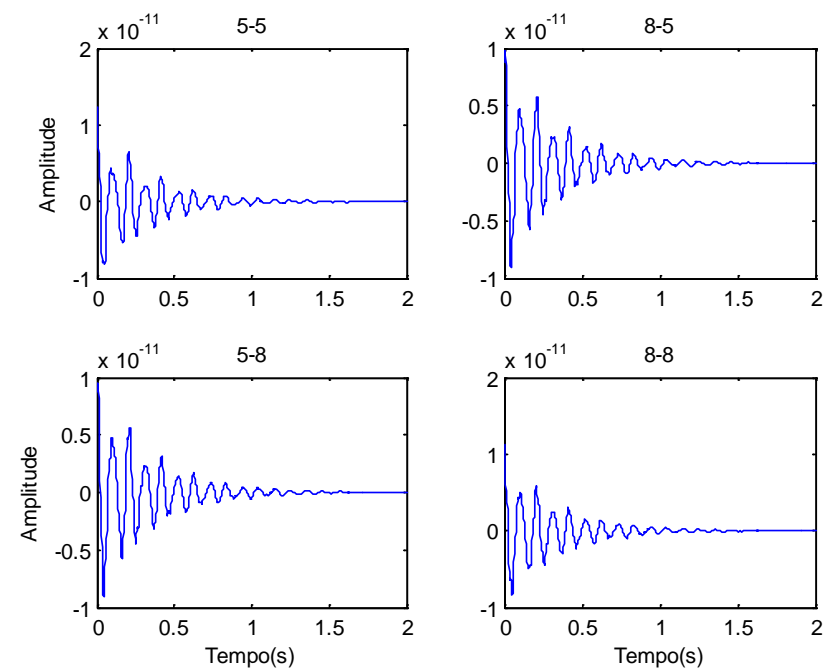

Figura A-17 FCs nos graus de liberdade 5 e 8 para uma entrada de ruído branco nos graus de liberdade 5 e 17, calculadas com Myfem Modal . 


\section{A.3 EXEMPLOS DE USO DO PROGRAMA PARA A ANÁLISE MODAL A PARTIR DE DADOS DE RESPOTA DO MODELO ESTRUTURAL.}

Nesta seção serão tratados alguns dos elementos do programa Myfem Modal para a análise modal a partir de dados da resposta do modelo estrutural, tais como: a estimativa de funções de correlação, estimativa de funções de densidade espectral, e a estimativa de funções de transmissibilidade multivariável. Também será mostrado um exemplo de estimativa de parâmetros modais (frequências de ressonância, formas modais e taxas de amortecimento modais) a partir do uso do método básico de seleção de picos. Outras metodologias para obtenção de parâmetros modais no domínio da frequência desenvolvidas em Myfem Modal não são abordadas neste apêndice.

Para fazer uma análise modal a partir de dados de resposta em Myfem Modal 2d, 0 primeiro passo é realizar uma análise dinâmica do modelo e obter as respostas nos diferentes graus de liberdade. Dessa forma, foi realizada uma análise dinâmica sobre o mesmo modelo estrutural que tem sido usado anteriormente (Figura A-3). Assim, foi definido um carregamento dinâmico com densidade espectral constante (ruído branco) localizado nos graus de liberdade 11 e 14 no modelo estrutural. Para isso, usaram-se os métodos noiseWG, para a geração de uma excitação com densidade espectral constante, addloadPDina para o estabelecimento de um carregamento dinâmico pontual, analysisDL para a análise dinâmica sob o carregamento e RecordNodeDL para a obtenção da resposta dinâmica nos graus de liberdade.

Para o método noiseWG, precisam-se dos argumentos de: variância do ruído branco, frequência de amostragem e tempo total do carregamento; para o método addloadPDina, o número do nó da localização do carregamento e a informação do carregamento colocada no respectivo grau de liberdade do nó; para o método RecordNodeDL, número do nó e o grau de liberdade do nó. No caso da análise dinâmica também foi considerada uma condensação estática da matriz de rigidez para uma análise só dos graus de liberdade verticais, como foi realizado no exemplo anterior. Mostra-se na Figura A-18 uma descrição do procedimento em Myfem Modal 2d. 


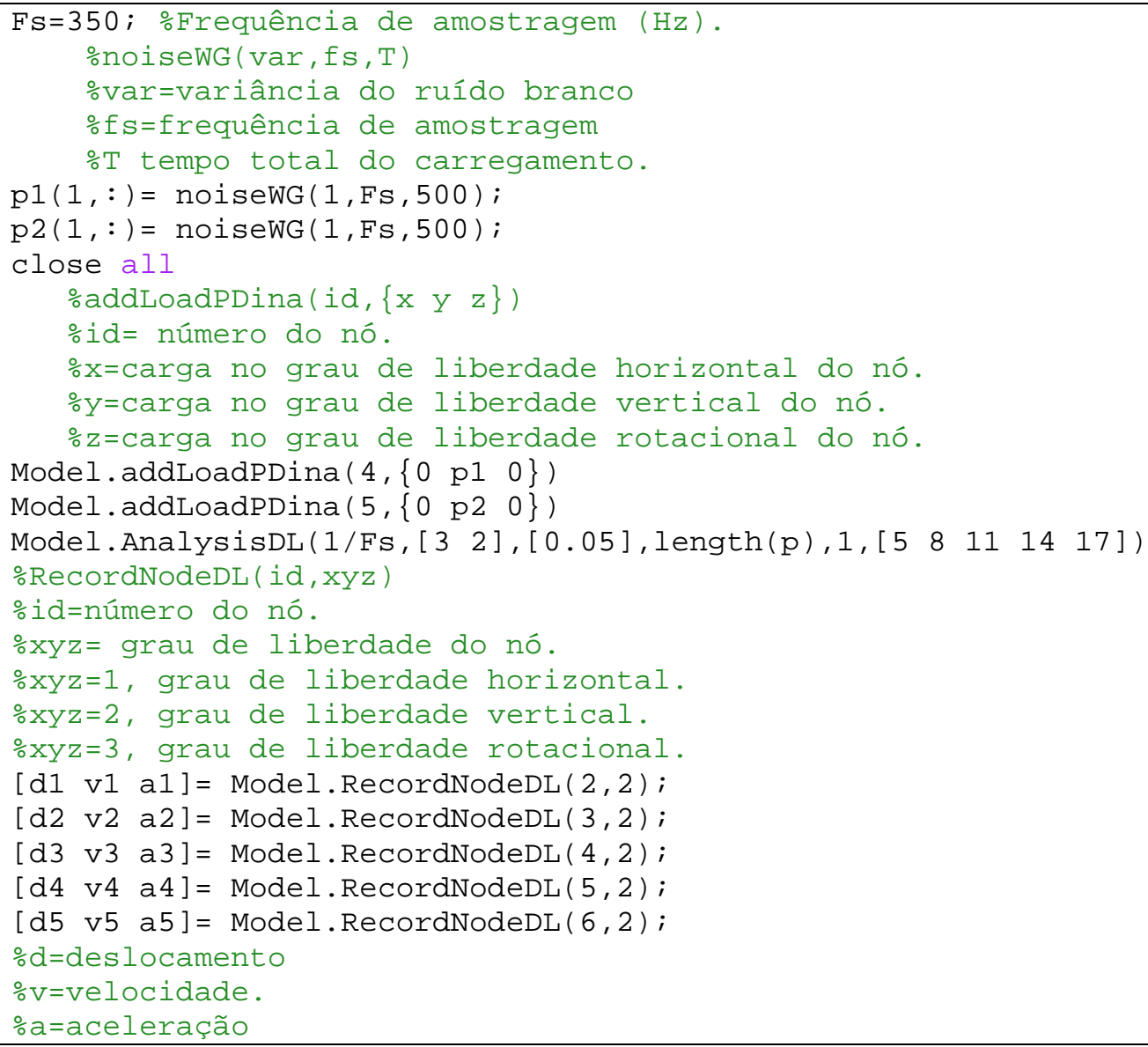

Figura A-18 Definição da análise dinâmica para a análise modal a partir de dados de resposta.

Como é notado na Figura A-18, definiu-se uma frequência de amostragem para a análise dinâmica de 350hz. Para o carregamento foi estabelecido como do tipo ruído branco com uma variância de 1 e um tempo total de 500seg, localizado nos nós 4 e 5, nos grau de liberdade verticais. Também, foram extraídas depois da análise dinâmica os deslocamentos, velocidades e acelerações dos graus de liberdade verticais dos nós 2,3,4,5 e 6 .

No procedimento da Figura A-18 quando é invocado o método noiseWG mostra-se automaticamente uma janela com a informação do sinal gerado, que consiste em: o sinal no tempo, a função de correlação do sinal e a densidade espectral de potência. Assim, no caso de ruído branco deve-se ter uma função de correlação da forma do impulso, com só o valor da variância do sinal localizada no tempo zero da função de correlação e uma densidade espectral constante. A janela saída do método noiseWG é da forma como mostrada na Figura A-19 . 

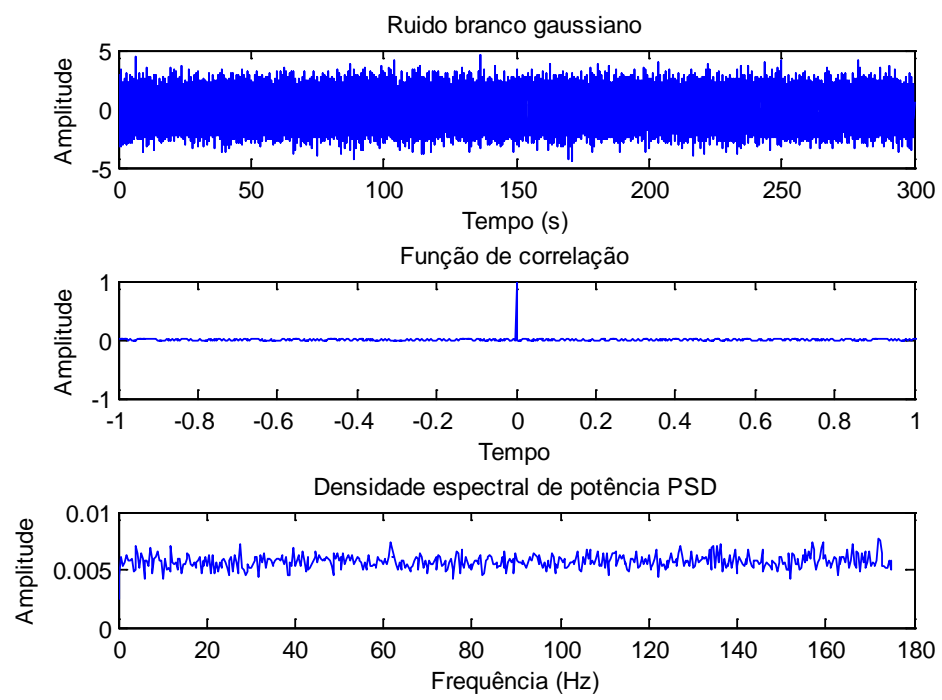

Figura A-19 Informação do método noiseWG sobre o ruído branco gerado.

\section{A.3.1 Funções de correlação de dados de resposta.}

Para a estimativa das funções de correlação a partir dos dados de resposta foram construídos os métodos em Myfem Modal 2d. chamados como Mycorr1, Mycorr2 e MyRD, os quais contêm as metodologias do método direto, método do periodograma médio e método de decremento aleatório, respectivamente. O método Mycorr1 requer principalmente dos argumentos de: os sinais de resposta, frequência de amostragem dos sinais e o tempo máximo da função de correlação. Também dentro dos argumentos pode-se optar pelo cálculo da função de correlação de dois lados ou de um só lado e normalizar a 1. Para o método Mycorr2, além de usar os mesmos parâmetros para Mycorr1, se precisa dos parâmetros de porcentagem de sobreposição das janelas e o tamanho e tipo de janela. No método MyRD é necessário selecionar o tipo de condição para seleção dos segmentos do sinal, entre as condições de: passagem de nível, passagem de zero, extremos locais e passagem de pontos positivos . Na Figura A-20 mostra-se uma descrição do procedimento realizado com os métodos Mycorr1, Mycorr2 e MyRD. 


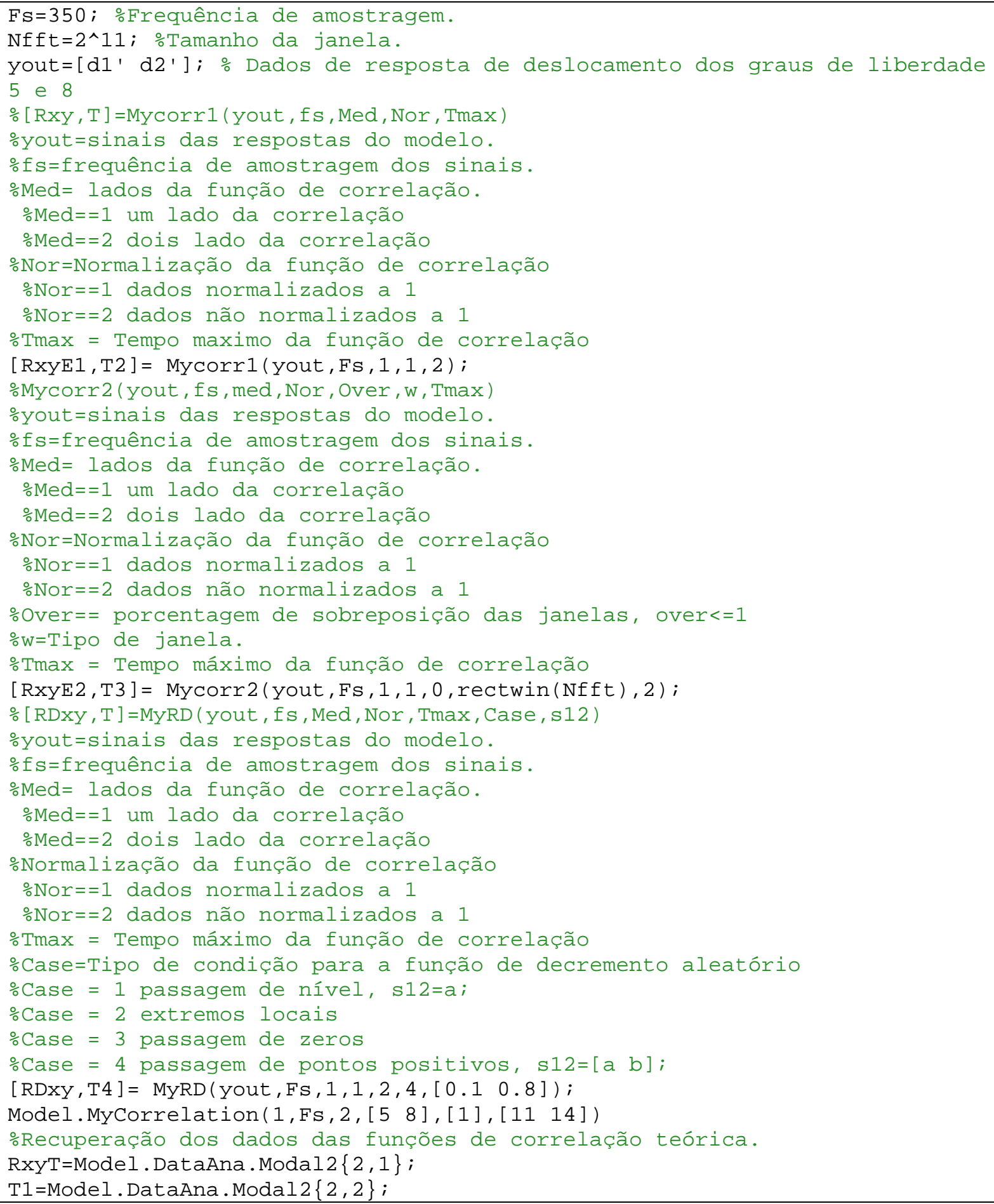

Figura A-20 Cálculo de funções de correlação de dados de resposta do modelo estrutural com Myfem Modal 2d.

Na Figura A-20, realiza-se o cálculo das funções de correlação para as respostas dinâmicas em deslocamentos dos graus de liberdade 5 e 8 do modelo, por meio dos métodos Mycorr1, Mycorr2 e MyRD. Os resultados são salvados nas variáveis RxyE1, 
RxyE2 e $R \boldsymbol{D} x \boldsymbol{y}$, que contêm as estimações das funções de correlação $\hat{R}_{x_{5} x_{5}}, \hat{R}_{x_{5} x_{8}}, \hat{R}_{x_{8} x_{5}}$, $\hat{R}_{x_{8} x_{B}}$. Para o caso das funções de decremento aleatório, foi selecionada a condição de passagem de pontos positivos entre os limites de 0.1 e 0.8 da máxima amplitude do sinal. Também se faz a avaliação da função de correlação teórica pelo método MyCorrelation. Assim, mostra-se na Figura A-21 uma comparação entre a estimação da função de correlação normalizada $\hat{R}_{x_{5} x_{8}}$, por meio do método direto, periodograma médio e decremento aleatório e a função de correlação teórica, na que se pode notar que todos os métodos usados tendem aproximar adequadamente a função de correlação teórica.

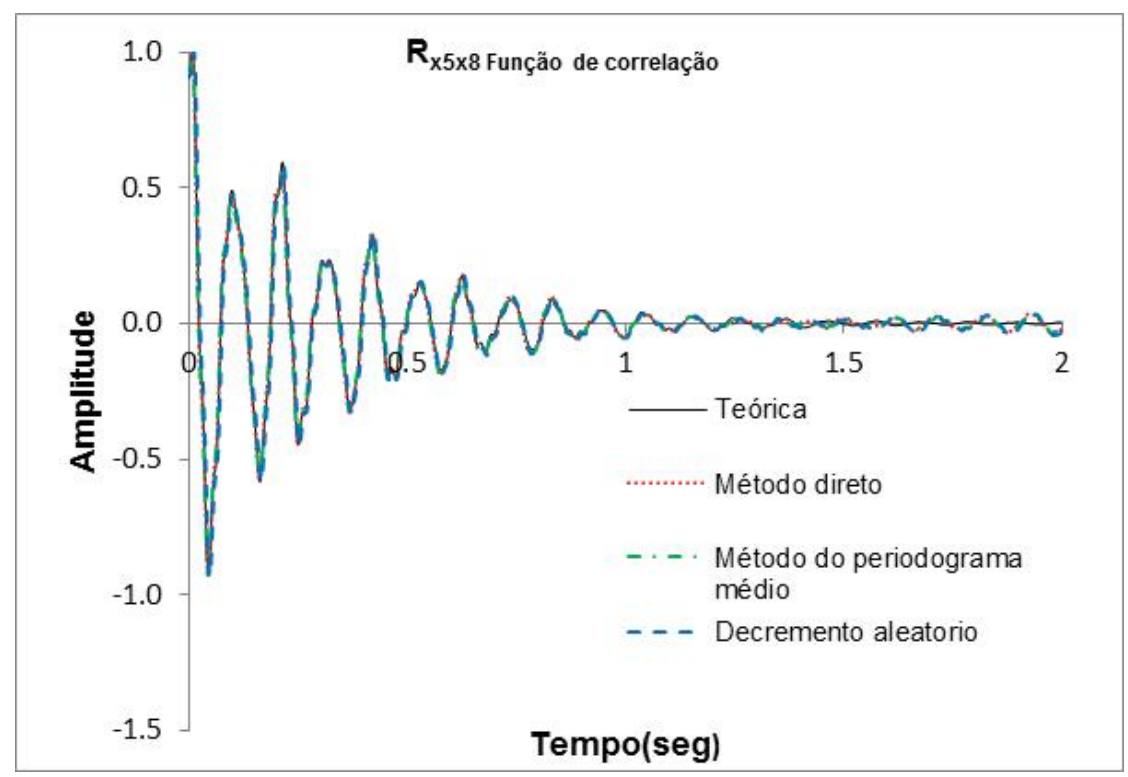

Figura A-21 Comparação das funções de correlação pelos métodos: direto, periodograma médio e decremento aleatório, com a função de correlação teórica.

\section{A.3.2 Funções de densidade espectral de dados de resposta}

Para a estimativa das funções de densidade espectral de dados de resposta do modelo estrutural foram desenvolvidos os métodos em Myfem Modal $2 d$. chamados de MyPSDWelch e MyPSDBT, os quais contêm os métodos de estimação de Welch e o método de Blackman e Tukey, respectivamente. O método MyPSDWelch requer dos argumentos seguintes: sinais de resposta do modelo estrutural, porcentagem de sobreposição das janelas, tamanho e tipo de janela, tamanho da transformada de Fourier e a frequência de amostragem. Para MyPSDBT precisa-se : das funções de correlação, o tipo de janela, o tamanho da transforma de Fourier, a frequência de amostragem e estabelecer o tipo de função de correlação, seja de dois lados ou de um só lado. Na Figura A-22 mostra-se a descrição do procedimento realizado com os métodos MyPSDWelch e MyPSDBT. 


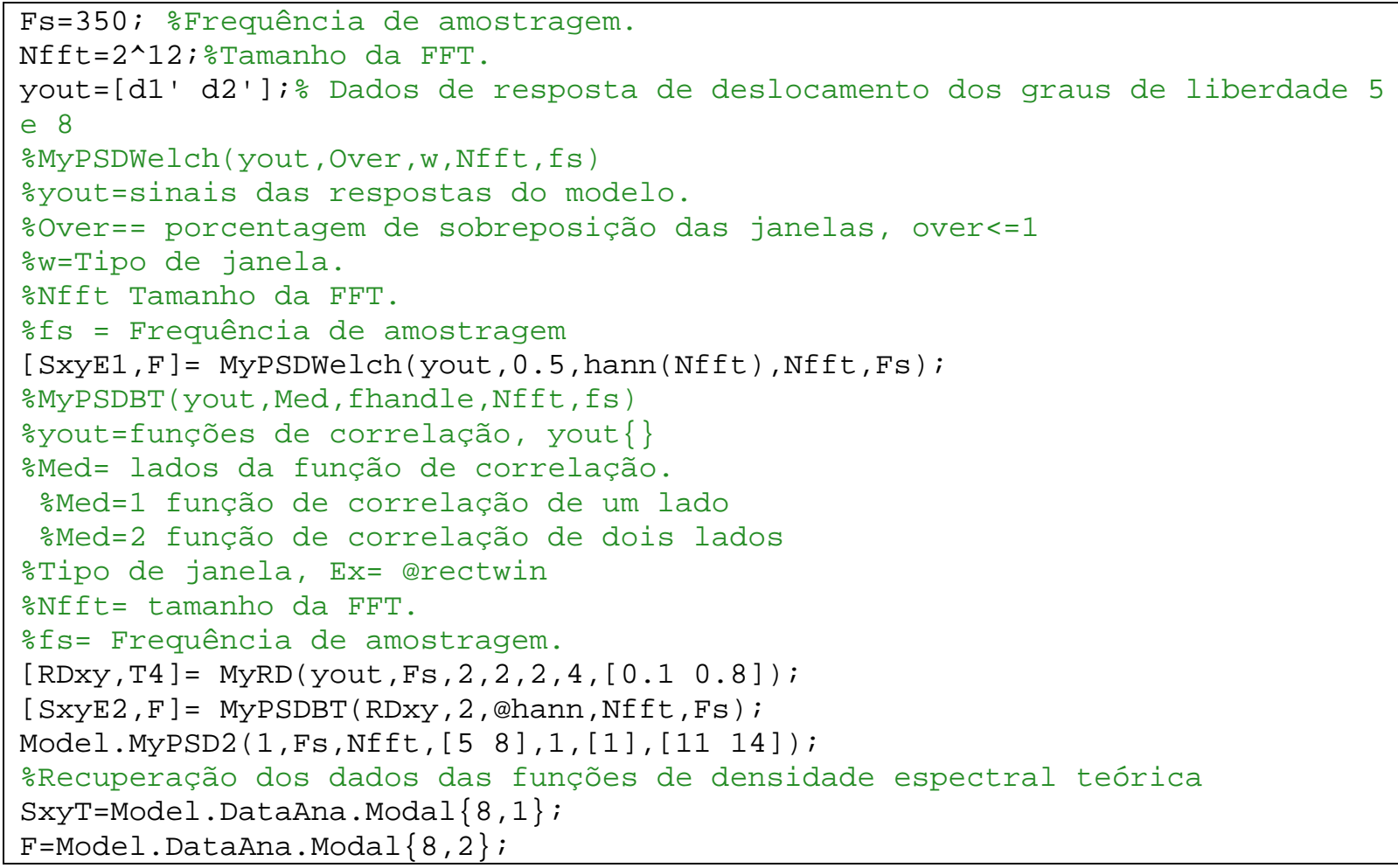

Figura A-22 Cálculo de funções de densidade espectral de dados de resposta do modelo estrutural com Myfem Modal $2 d$.

Como é notado na Figura A-22, a partir de usar os métodos MyPSDWelch e MyPSDBT foram avaliadas as funções de densidade espectral das respostas em deslocamento dos graus de liberdade 5 e 8, produto de uma excitação no grau de liberdade 11. Os resultados são salvados nas variáveis SxyE1 e SxyE2, os quais contém as estimativa das densidades espectrais $\hat{S}_{x_{5} x_{5}}, \hat{S}_{x_{5} x_{8}}, \hat{S}_{x_{8} x_{5}}, \hat{S}_{x_{8} x_{8}}$. Para as estimativas das densidades espectrais pelo método de Blackman y Tukey foram usadas as funções de correlação estimadas por meio do método de decremento aleatório com uma condição de passagem de pontos positivos. Assim, mostra-se na Figura A-23 uma comparação entre as estimativas da função de densidade espectral $\hat{S}_{x_{5} x_{5}}$ por meio dos métodos de Welch e Blackman e Tukey, e a função de densidade espectral teórica. 


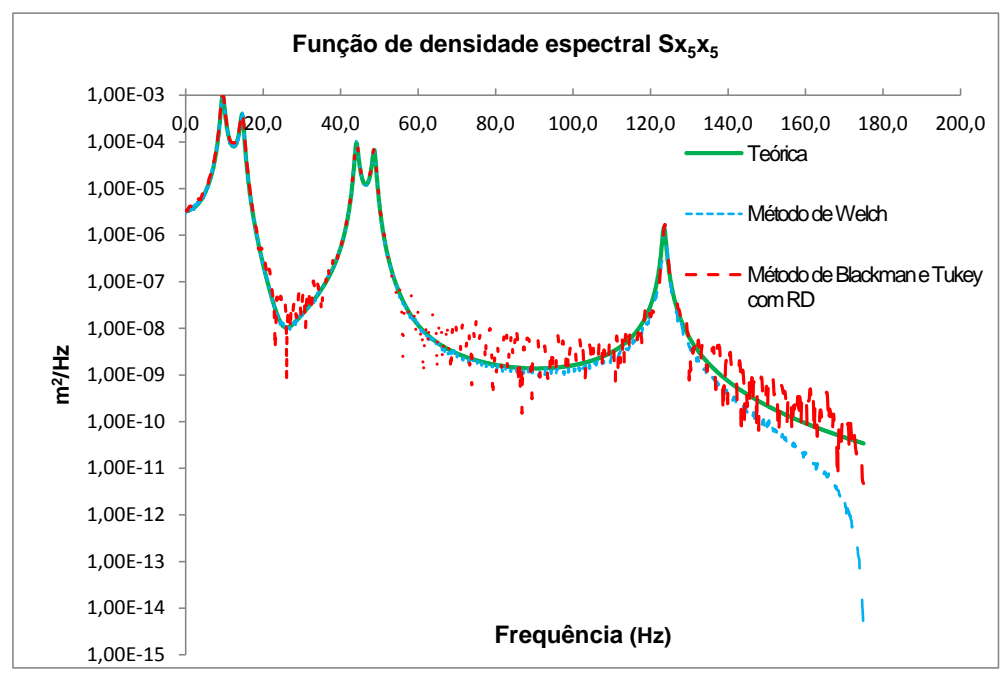

Figura A-23 Comparação da função de densidade espectral teórica com as funções estimativas dos métodos de: Welch e Blackman e Tukey com as funções de decremento aleatório.

\section{A.3.3 Funções de transmissibilidade de dados de resposta.}

Uma estimativa das funções de transmissibilidade para os dados de resposta do modelo estrutural é efetuada através de considerar a Equação (2-68), da seguinte forma.

$$
\left.\left[T^{a}{ }_{x_{u} x_{k}}(i \omega)\right]=\left[\hat{S}_{x_{u} x_{k}}(i \omega)\right] \hat{S}_{x_{k} x_{k}}(i \omega)\right]^{+}
$$

Em que, $\left\lfloor T^{a}{ }_{x_{u} x_{k}}(i \omega)\right\rfloor$ são as funções de transmissibilidade entre os graus de liberdade $\boldsymbol{u}$ com relação aos graus de liberdades $\boldsymbol{k}$ considerando um carregamento dinâmico nos graus de liberdade a. A partir de considerar a Equação (A-1) é desenvolvido em Myfem Modal $2 d$. o método chamado de MytransWelch que avalia as funções de transmissibilidade $\left[T_{x_{u} x_{k}}^{a}(i \omega)\right]$, realizando o cálculo das matrizes de funções de densidades espectrais $\left[S_{x_{u} x_{k}}(i \omega)\right]$ e $\left[S_{x_{k} x_{k}}(i \omega)\right]$ usando o método de Welch. Os parâmetros necessários para o método MytransWelch, são: sinais de resposta do modelo estrutural, definição dos graus de liberdade $\boldsymbol{k}$ e $\boldsymbol{u}$, a porcentagem de sobreposição das janelas, tamanho e tipo de janela, tamanho da transformada de Fourier e a frequência de amostragem. Na Figura A-24 mostrase uma descrição do uso do método MytransWelch para a avaliação das funções de transmissibilidade multivariável .

Como exemplo para o cálculo de funções de transmissibilidade em Myfem Modal $2 d$. é mostrado na Figura A-24 o computo da transmissibilidade entre os graus de liberdades $\boldsymbol{u}=$ $5,8,17$ do modelo estrutural, e os graus de liberdade $k=11,14$, com um carregamento dinâmico nos graus de liberdade $\mathbf{a}=11,14$. A matriz de transmissibilidade resultante será dada por: 


$$
\left[T^{a}{ }_{x_{u} x_{k}}(i \omega)\right]=\left[\begin{array}{cc}
T_{x_{5} x_{11}}^{a}(i \omega) & T_{x_{5} x_{14}}^{a}(i \omega) \\
T_{x_{8} x_{11}}^{a}(i \omega) & T_{x_{8} x_{14}}^{a}(i \omega) \\
T_{x_{17} x_{11}}^{a}(i \omega) & T^{a}{ }_{x_{17} x_{14}}(i \omega)
\end{array}\right]
$$

Nesse exemplo considera-se um carregamento dinâmico do tipo ruído branco aplicado nos graus de liberdade $\mathbf{a}$, assim, a partir das respostas em deslocamento nos diferentes graus de liberdade da análise dinâmica avaliam-se com MytransWelch as funções de transmissibilidade $\left[T^{a}{ }_{x_{u} x_{k}}(\boldsymbol{i} \boldsymbol{\omega})\right]$. Mostra-se na Figura A-25 uma comparação entre a função de transmissibilidade $T^{a}{ }_{x_{8} x_{11}}(i \omega)$ calculada a partir de dados de resposta, e a função de transmissibilidade teórica avaliada com o método Mytransm de Myfem Modal $2 d$.

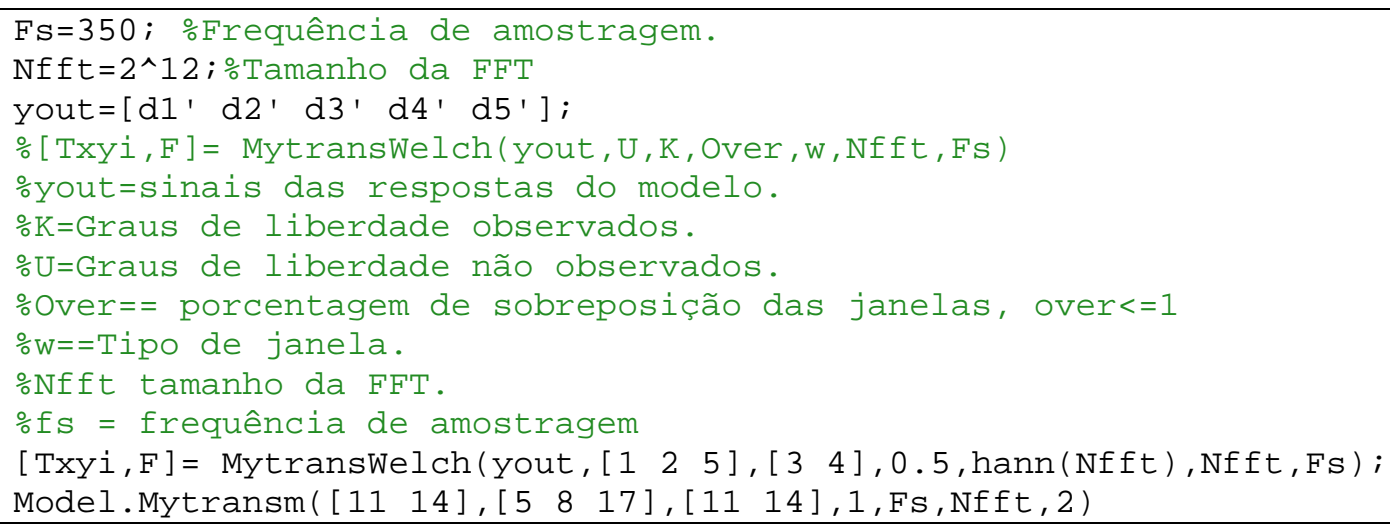

Figura A-24 Cálculo de funções de transmissibilidade de dados de resposta com Myfem Modal 2d. .

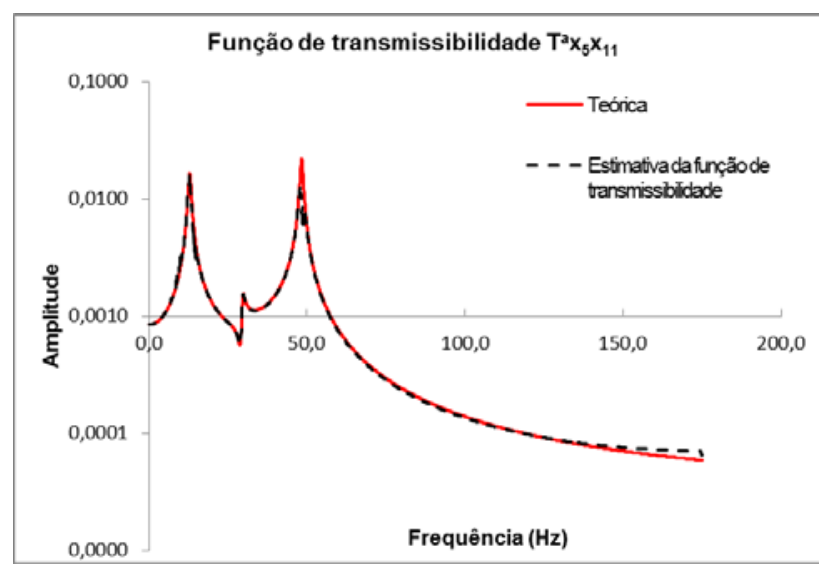

Figura A-25 Comparação da função de transmissibilidade estimada a partir de dados de resposta do modelo estrutural com a função de transmissibilidade teórica com Myfem Modal 2d. . 


\section{A.3.4 Uso do método de seleção de picos para avaliação de} parâmetros modais.

O método de seleção de picos desenvolvidos em Myfem Modal 2d. para a avaliação de parâmetros modais (frequências de ressonância, formas modais e taxas de amortecimento) possui três rotinas diferentes chamadas de MyANPSD, Mytfmodos, e Myamortajuste. O método MyANPSD define a média dos auto espectros da matriz de densidade espectral, para a seleção das frequências de ressonância do sistema, Mytfmodos avalia as funções de transmissibilidade para todos os graus de liberdade com respeito em um ponto de referência e é usado para avaliação das formas modais, e o método Myamortajuste estabelece um ajuste do espectro teórico de um só grau de liberdade na vizinhança de uma frequência de ressonância e é usado para avaliação da porcentagem de amortecimento modal.

Os parâmetros necessários para o funcionamento dos diferentes métodos são: para o método MyANPSD, a matriz de densidade espectral, para Mytfmodos precisa dos sinais de resposta do modelo estrutural, o número do grau de liberdade de referência, o tipo de janela, o tamanho da transforma de Fourier, a frequência de amostragem e o valor de uma frequência de ressonância. O último parâmetro é usado para extrair o modo da frequência de ressonância determinada. Para Myamortajuste é necessário o tipo de espectro para o ajuste, seja para o deslocamento, a velocidade ou a aceleração, o espectro com suas abscissas, o valor de uma frequência de ressonância e os limites superior e inferior da frequência de ressonância para estabelecer a vizinhança do ajuste.

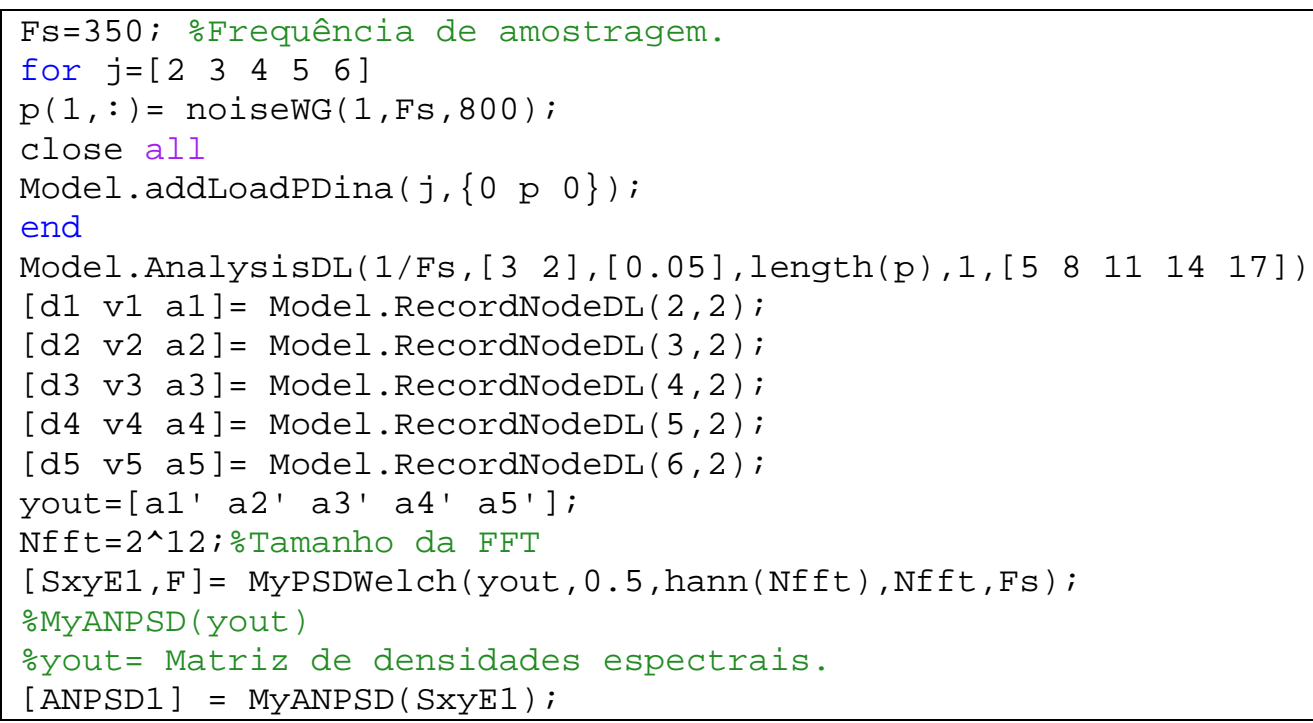

Figura A-26 Exemplo de uso do método de seleção de picos em Myfem Modal $2 d$. 
Para exemplificar o uso do método de seleção de picos tem sido realizada uma análise dinâmica do modelo estrutural da Figura A-3 a partir de estabelecer um carregamento do tipo ruído branco em todos os graus de liberdade livres do modelo, seguidamente as respostas dinâmicas da análise são coletadas para posteriormente aplicar os métodos MyANPSD, Mytfmodos, e Myamortajuste. Dessa forma, mostra-se na Figura A-27 uma descrição do procedimento realizado com Myfem Modal 2d. .

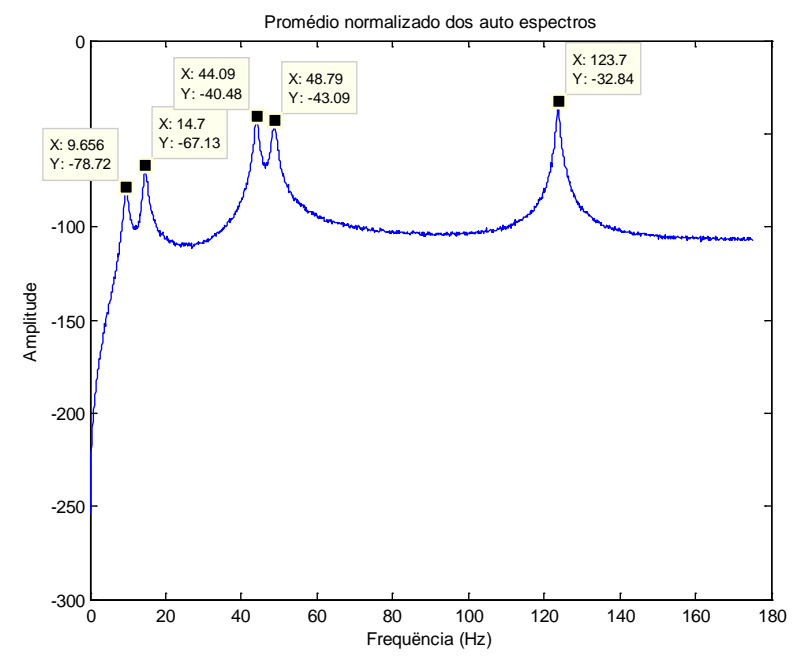

Figura A-27 Média normalizado dos auto-espectros da matriz de densidade espectral.

Na Figura A-27 são indicados os valores dos picos do gráfico da média dos autoespectros que correspondem às ressonâncias do modelo. Os valores dos picos são comparados com os valores teóricos das frequências amortecidas na Tabela A-4.

Tabela A-3 Frequências de ressonância do modelo (teórico-identificado)

\begin{tabular}{ccc}
\hline $\begin{array}{c}\text { Fd Teórico } \\
(\mathrm{Hz})\end{array}$ & $\begin{array}{c}\text { Fd identificado } \\
(\mathrm{Hz})\end{array}$ & Erro(\%) \\
\hline 9,539 & 9,650 & $1,165 \%$ \\
14,616 & 14,700 & $0,577 \%$ \\
44,049 & 44,090 & $0,093 \%$ \\
48,700 & 48,790 & $0,185 \%$ \\
123,486 & 123,700 & $0,174 \%$ \\
\hline
\end{tabular}

Para estimação das formas modais o método usado é Mytfmodos, assim, mostra-se uma descrição do procedimento na Figura A-28. 


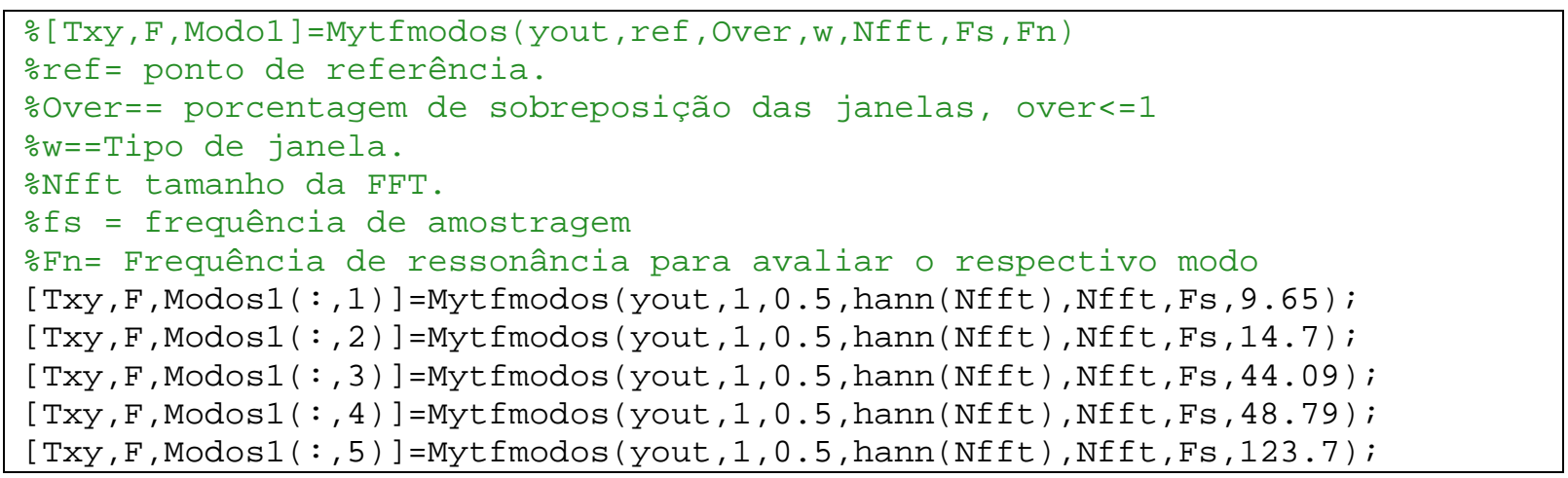

Figura A-28 Uso do método Mytfmodos em Myfem Modal 2d. .

No procedimento da Figura A-28 quando é chamado o método Mytfmodos mostrase automaticamente uma janela com a informação em amplitude e fase das diferentes funções de transmissibilidade nos graus de liberdade com respeito ao ponto de referência. A janela saída do método Mytfmodos é da forma mostrada na Figura A-29.

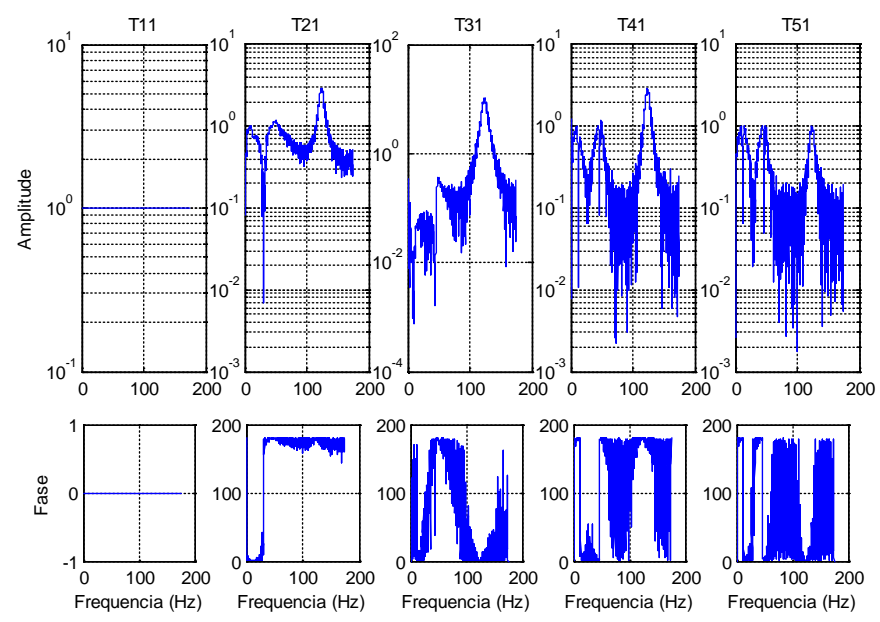

Figura A-29 Informação do método Mytfmodos de Myfem Modal 2d. sobre as funções de transmissibilidade.

As formas modais identificadas são mostradas na Tabela A-5 e se estabelece uma comparação com os modos teóricos de vibração (Tabela A-3) a partir de usar o indicador MAC (Equação 3-47), os resultados são mostrados na Tabela A-6.

Tabela A-4 Formas modais identificadas.

\begin{tabular}{cccccc}
\hline GL & Modo1 & Modo2 & Modo3 & Modo4 & Modo5 \\
\hline 5 & 1,000 & 1,000 & 1,000 & 1,000 & 1,000 \\
8 & 1,002 & 0,730 & $-1,007$ & $-1,183$ & $-2,961$ \\
11 & $-0,004$ & 0,053 & $-0,009$ & $-0,357$ & 10,198 \\
14 & $-0,990$ & 0,711 & 0,992 & $-1,140$ & $-2,963$ \\
17 & $-0,989$ & 0,982 & $-0,994$ & 0,973 & 1,007 \\
\hline
\end{tabular}

$\mathrm{GL}=\mathrm{Grau}$ de liberdade 
Tabela A-5 Comparação de formas modais teóricas e identificadas.

\begin{tabular}{cccccc}
\hline & & \multicolumn{5}{c}{ MAC } \\
T/I & Modo1 & Modo2 & Modo3 & Modo4 & Modo5 \\
\hline Modo1 & 1,000 & 0,000 & 0,000 & 0,000 & 0,000 \\
Modo2 & 0,000 & 1,000 & 0,000 & 0,005 & 0,008 \\
Modo3 & 0,000 & 0,000 & 1,000 & 0,000 & 0,000 \\
Modo4 & 0,000 & 0,005 & 0,000 & 1,000 & 0,046 \\
Modo5 & 0,000 & 0,008 & 0,000 & 0,045 & 1,000 \\
\hline
\end{tabular}

T=Teórico, I=Identificado

Finalmente, mostra-se na Figura A-30 uma descrição do procedimento realizado com uso do método Myamortajuste para avaliação das taxas de amortecimento modais.

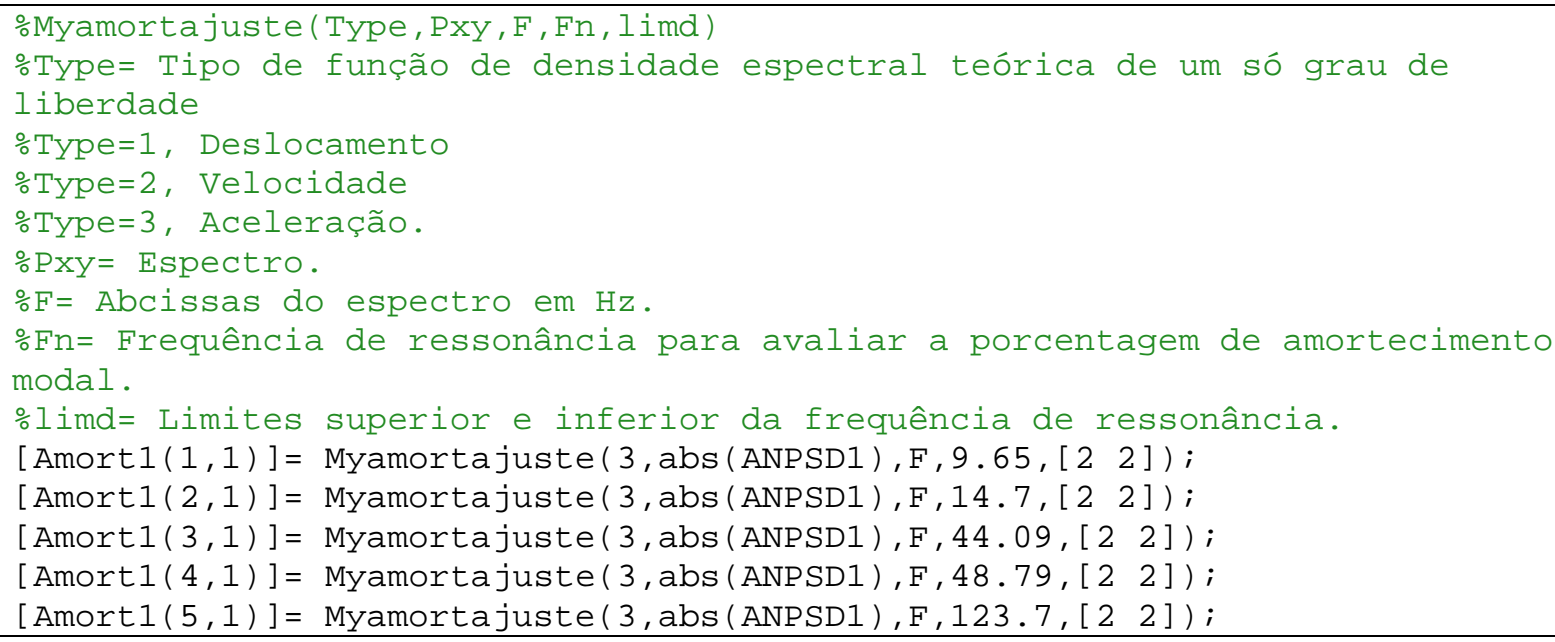

Figura A-30 Uso do método Myamortajuste em Myfem Modal 2d. .

No procedimento da Figura A-31 é usado à média normalizada dos auto-espectros da matriz de densidade espectral, para avaliar as taxas de amortecimento. Mostra-se a seguir um exemplo da janela de informação do método Myamortajuste, que especifica as características do ajuste realizado ( Figura A-31). 

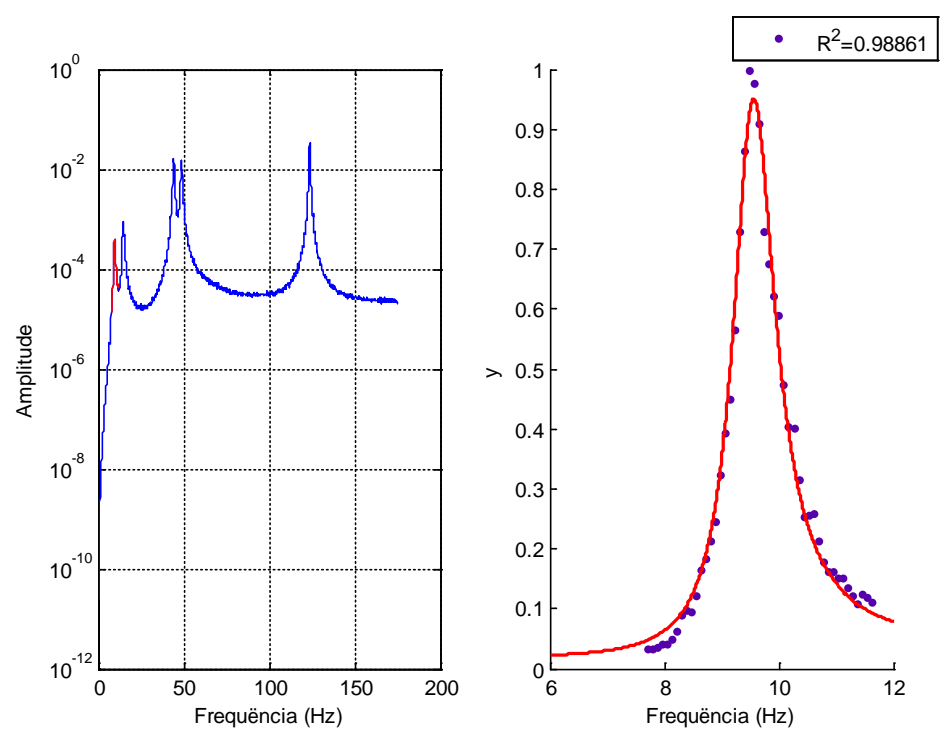

Figura A-31 Informação do método Myamortajuste de Myfem Modal 2d.

Da aplicação da técnica de ajuste do espectro analítico que corresponde a um sistema de um só grau de liberdade, por meio do método Myamortajuste de Myfem Modal $2 d$ foi possível fazer uma estimativa das taxas de amortecimentos dos diferentes modos de vibração, que são revelados na Tabela A-7 ao fazer uma comparação com as taxas de amortecimento teóricas.

Tabela A-6 Taxas de amortecimentos (teórico-identificado)

\begin{tabular}{ccc}
\hline$\xi$ Teórico(\%) & $\xi$ Identificado(\%) & Erro(\%) \\
\hline $5,00 \%$ & $4,76 \%$ & $4,74 \%$ \\
$3,27 \%$ & $3,68 \%$ & $12,69 \%$ \\
$1,08 \%$ & $1,05 \%$ & $3,31 \%$ \\
$0,98 \%$ & $0,93 \%$ & $5,58 \%$ \\
$0,39 \%$ & $0,32 \%$ & $16,79 \%$ \\
\hline
\end{tabular}

Desta forma, tem sido mostrado um exemplo da estimativa de parâmetros modais a partir dos dados da resposta de um modelo estrutural, por meio do uso da ferramenta proposta para a análise modal Myfem Modal $2 d$. O método escolhido para esse exemplo foi o método de seleção de picos, o qual para o exemplo realizado mostra um apropriado desempenho na estimativa dos parâmetros modais, devido principalmente a que são cumpridas as diferentes suposições em que é baseada está metodologia, que são: a excitação pode excitar todos os modos do sistema com um espectro constante (ruído branco), existe uma adequada distribuição do carregamento e os modos de vibração são relativamente bem separados com ausência de ruído no espectro. 


\section{A.4 CONSIDERAÇÕES FINAIS}

O presente apêndice apresenta uma nova ferramenta computacional para a análise modal teórica e operacional de modelos estruturais tipo pórticos no plano, onde se descrevem de forma geral as diferentes utilidades contidas no programa e se estabelecem variados tipos de exemplos com o intuito de fazer uma introdução ao uso do programa.

Salientam-se diferentes características do programa:

a) Têm-se ao alcance diferentes ferramentas da análise modal teórico para validar a implementação dos algoritmos e técnicas para estimativa de características dinâmicas a partir de dados de resposta do modelo estrutural, bem como para novos algoritmos que possam surgir.

b) A possibilidade de simular dados experimentais advindos do mesmo modelo estrutural que poder iam-se obter em uma medição de vibrações real.

c) A possibilidade de simular variados tipos de condições na identificação modal como o tipo de excitação e localização da excitação.

d) As diferentes rotinas até o momento desenvolvidas para estimativa de características dinâmicas de dados de resposta em Myfem Modal 2d. podem ser também utilizadas para dados saídos de vibrações reais.

Da mesma forma, alguns aspectos podem ser motivo de trabalhos futuros para a melhora do programa, tais como: extensão para a análise de estruturas tipo pórtico em $3 d$ e estruturas planares e a mudança de linguagem de programação devido às limitações de recurso de memória que tem o Matlab que tornaria difícil a análise de modelos estruturais de maior tamanho. 